EUROPEAN ORGANIZATION FOR NUCLEAR RESEARCH

CERN / PPE 96-186

December 13 th, 1996

\title{
Studies of Quantum Chromodynamics \\ with the ALEPH Detector
}

\section{The ALEPH Collaboration}

\begin{abstract}
Previously published and as yet unpublished QCD results obtained with the ALEPH detector at LEP1 are presented. The unprecedented statistics allows detailed studies of both perturbative and non-perturbative aspects of strong interactions to be carried out using hadronic $\mathrm{Z}$ and tau decays. The studies presented include precise determinations of the strong coupling constant, tests of its flavour independence, tests of the SU(3) gauge structure of QCD, study of coherence effects, and measurements of single-particle inclusive distributions and two-particle correlations for many identified baryons and mesons.
\end{abstract}

To appear in Physics Reports 
R. Barate, D. Buskulic, D. Decamp, P. Ghez, C. Goy, J.-P. Lees, A. Lucotte, M.-N. Minard, J.-Y. Nief, P. Odier, B. Pietrzyk

Laboratoire de Physique des Particules (LAPP), IN ${ }^{2} P^{3}$-CNRS, 74019 Annecy-le-Vieux Cedex, France

M.P. Casado, M. Chmeissani, P. Comas, J.M. Crespo, M. Delfino, E. Fernandez, M. Fernandez-Bosman, Ll. Garrido, ${ }^{15}$ A. Juste, M. Martinez, S. Orteu, C. Padilla, I.C. Park, A. Pascual, J.A. Perlas, I. Riu, F. Sanchez, F. Teubert

Institut de Fisica d'Altes Energies, Universitat Autonoma de Barcelona, 08193 Bellaterra (Barcelona), $\operatorname{Spain}^{7}$

A. Colaleo, D. Creanza, M. de Palma, G. Gelao, G. Iaselli, G. Maggi, M. Maggi, N. Marinelli, S. Nuzzo, A. Ranieri, G. Raso, F. Ruggieri, G. Selvaggi, L. Silvestris, P. Tempesta, A. Tricomi, ${ }^{3}$ G. Zito

Dipartimento di Fisica, INFN Sezione di Bari, 70126 Bari, Italy

X. Huang, J. Lin, Q. Ouyang, T. Wang, Y. Xie, R. Xu, S. Xue, J. Zhang, L. Zhang, W. Zhao Institute of High-Energy Physics, Academia Sinica, Beijing, The People's Republic of China ${ }^{8}$

D. Abbaneo, R. Alemany, A.O. Bazarko, P. Bright-Thomas, M. Cattaneo, F. Cerutti, H. Drevermann, R.W. Forty, M. Frank, R. Hagelberg, J. Harvey, P. Janot, B. Jost, E. Kneringer, J. Knobloch, I. Lehraus, T. Lohse, G. Lutters, P. Mato, A. Minten, R. Miquel, Ll.M. Mir, ${ }^{2}$ L. Moneta, T. Oest, ${ }^{20}$ A. Pacheco, J.-

F. Pusztaszeri, F. Ranjard, P. Rensing, ${ }^{12}$ G. Rizzo, L. Rolandi, D. Schlatter, M. Schmelling, ${ }^{24}$ M. Schmitt, O. Schneider, W. Tejessy, I.R. Tomalin, A. Venturi, H. Wachsmuth, A. Wagner

European Laboratory for Particle Physics (CERN), 1211 Geneva 23, Switzerland

Z. Ajaltouni, A. Barrès, C. Boyer, A. Falvard, C. Ferdi, P. Gay, C . Guicheney, P. Henrard, J. Jousset, B. Michel, S. Monteil, J-C. Montret, D. Pallin, P. Perret, F. Podlyski, J. Proriol, P. Rosnet, J.-M. Rossignol Laboratoire de Physique Corpusculaire, Université Blaise Pascal, IN ${ }^{2} P^{3}$-CNRS, Clermont-Ferrand, 63177 Aubière, France

T. Fearnley, J.B. Hansen, J.D. Hansen, J.R. Hansen, P.H. Hansen, B.S. Nilsson, B. Rensch, A. Wäänänen Niels Bohr Institute, 2100 Copenhagen, Denmark ${ }^{9}$

G. Daskalakis, A. Kyriakis, C. Markou, E. Simopoulou, I. Siotis, A. Vayaki, K. Zachariadou Nuclear Research Center Demokritos (NRCD), Athens, Greece

A. Blondel, G. Bonneaud, J.C. Brient, P. Bourdon, A. Rougé, M. Rumpf, A. Valassi, ${ }^{6}$ M. Verderi, H. Videau Laboratoire de Physique Nucléaire et des Hautes Energies, Ecole Polytechnique, IN ${ }^{2} P^{3}-C N R S, 91128$ Palaiseau Cedex, France

D.J. Candlin, M.I. Parsons Department of Physics, University of Edinburgh, Edinburgh EH9 3JZ, United Kingdom ${ }^{10}$

E. Focardi, ${ }^{21}$ G. Parrini

Dipartimento di Fisica, Università di Firenze, INFN Sezione di Firenze, 50125 Firenze, Italy

M. Corden, C. Georgiopoulos, D.E. Jaffe

Supercomputer Computations Research Institute, Florida State University, Tallahassee, FL 323064052, USA 13,14

A. Antonelli, G. Bencivenni, G. Bologna, ${ }^{4}$ F. Bossi, P. Campana, G. Capon, D. Casper, V. Chiarella, G. Felici, P. Laurelli, G. Mannocchi, ${ }^{5}$ F. Murtas, G.P. Murtas, L. Passalacqua, M. Pepe-Altarelli

Laboratori Nazionali dell'INFN (LNF-INFN), 00044 Frascati, Italy

L. Curtis, S.J. Dorris, A.W. Halley, I.G. Knowles, J.G. Lynch, V. O’Shea, C. Raine, P. Reeves, J.M. Scarr, K. Smith, P. Teixeira-Dias, A.S. Thompson, E. Thomson, F. Thomson, R.M. Turnbull

Department of Physics and Astronomy, University of Glasgow, Glasgow G12 8QQ,United Kingdom ${ }^{10}$

U. Becker, O. Buchmüller, C. Geweniger, G. Graefe, P. Hanke, G. Hansper, H. Hepp, V. Hepp, E.E. Kluge, A. Putzer, M. Schmidt, J. Sommer, H. Stenzel, K. Tittel, S. Werner, M. Wunsch 
R. Beuselinck, D.M. Binnie, W. Cameron, P.J. Dornan, M. Girone, S. Goodsir, E.B. Martin, A. Moutoussi, J. Nash, J.K. Sedgbeer, A.M. Stacey, M.D. Williams

Department of Physics, Imperial College, London SW7 2BZ, United Kingdom ${ }^{10}$

G. Dissertori, P. Girtler, D. Kuhn, G. Rudolph

Institut für Experimentalphysik, Universität Innsbruck, 6020 Innsbruck, Austria ${ }^{18}$

A.P. Betteridge, C.K. Bowdery, P. Colrain, G. Crawford, A.J. Finch, F. Foster, G. Hughes, T. Sloan, M.I. Williams

Department of Physics, University of Lancaster, Lancaster LA1 4YB, United Kingdom ${ }^{10}$

T. Barczewski, A. Galla, I. Giehl, A.M. Greene, C. Hoffmann, K. Jakobs, K. Kleinknecht, G. Quast, B. Renk,

E. Rohne, H.-G. Sander, H. Schmidt, F. Steeg, P. van Gemmeren, C. Zeitnitz

Institut für Physik, Universität Mainz, 55099 Mainz, Fed. Rep. of Germany ${ }^{16}$

J.J. Aubert, C. Benchouk, A. Bonissent, G. Bujosa, D. Calvet, J. Carr, P. Coyle, C. Diaconu, F. Etienne,

N. Konstantinidis, O. Leroy, P. Payre, D. Rousseau, M. Talby, A. Sadouki, M. Thulasidas, K. Trabelsi Centre de Physique des Particules, Faculté des Sciences de Luminy, IN ${ }^{2} P^{3}$-CNRS, 13288 Marseille, France

M. Aleppo, F. Ragusa ${ }^{21}$

Dipartimento di Fisica, Università di Milano e INFN Sezione di Milano, 20133 Milano, Italy

R. Berlich, W. Blum, V. Büscher, H. Dietl, F. Dydak, ${ }^{21}$ G. Ganis, C. Gotzhein, H. Kroha, G. Lütjens, G. Lutz, W. Männer, H.-G. Moser, R. Richter, A. Rosado-Schlosser, S. Schael, R. Settles, H. Seywerd, R. St. Denis, H. Stenzel, W. Wiedenmann, G. Wolf

Max-Planck-Institut für Physik, Werner-Heisenberg-Institut, 80805 München, Fed. Rep. of Germany ${ }^{16}$

J. Boucrot, O. Callot, ${ }^{21}$ S. Chen, Y. Choi, ${ }^{26}$ A. Cordier, M. Davier, L. Duflot, J.-F. Grivaz, Ph. Heusse, A. Höcker, A. Jacholkowska, M. Jacquet, D.W. Kim, ${ }^{19}$ F. Le Diberder, J. Lefrançois, A.-M. Lutz, I. Nikolic, H.J. Park, ${ }^{19}$ M.-H. Schune, S. Simion, J.-J. Veillet, I. Videau, D. Zerwas Laboratoire de l'Accélérateur Linéaire, Université de Paris-Sud, IN $\mathrm{N}^{2} \mathrm{P}_{-} \mathrm{CNRS}, 91405$ Orsay Cedex, France

P. Azzurri, G. Bagliesi, G. Batignani, S. Bettarini, C. Bozzi, G. Calderini, M. Carpinelli, M.A. Ciocci, V. Ciulli, R. Dell'Orso, R. Fantechi, I. Ferrante, L. Foà, ${ }^{1}$ F. Forti, A. Giassi, M.A. Giorgi, A. Gregorio, F. Ligabue, A. Lusiani, P.S. Marrocchesi, A. Messineo, F. Palla, G. Sanguinetti, A. Sciabà, P. Spagnolo, J. Steinberger, R. Tenchini, G. Tonelli, ${ }^{25}$ C. Vannini, P.G. Verdini

Dipartimento di Fisica dell'Università, INFN Sezione di Pisa, e Scuola Normale Superiore, 56010 Pisa, Italy

G.A. Blair, L.M. Bryant, J.T. Chambers, Y. Gao, M.G. Green, T. Medcalf, P. Perrodo, J.A. Strong, J.H. von Wimmersperg-Toeller Department of Physics, Royal Holloway \& Bedford New College, University of London, Surrey TW20 OEX, United Kingdom ${ }^{10}$

V. Bertin, D.R. Botterill, R.W. Clifft, T.R. Edgecock, S. Haywood, P. Maley, P.R. Norton, J.C. Thompson, A.E. Wright

Particle Physics Dept., Rutherford Appleton Laboratory, Chilton, Didcot, Oxon OX11 OQX, United Kingdom ${ }^{10}$

B. Bloch-Devaux, P. Colas, S. Emery, W. Kozanecki, E. Lançon, M.C. Lemaire, E. Locci, P. Perez, J. Rander, J.-F. Renardy, A. Roussarie, J.-P. Schuller, J. Schwindling, A. Trabelsi, B. Vallage CEA, DAPNIA/Service de Physique des Particules, CE-Saclay, 91191 Gif-sur-Yvette Cedex, France ${ }^{17}$

S.N. Black, J.H. Dann, R.P. Johnson, H.Y. Kim, A.M. Litke, M.A. McNeil, G. Taylor Institute for Particle Physics, University of California at Santa Cruz, Santa Cruz, CA 95064, USA 22

A. Beddall, C.N. Booth, R. Boswell, C.A.J. Brew, S. Cartwright, F. Combley, I. Dawson, M.S. Kelly, M. Lehto, W.M. Newton, J. Reeve, L.F. Thompson 
Department of Physics, University of Sheffield, Sheffield S3 7RH, United Kingdom ${ }^{10}$

A. Böhrer, S. Brandt, G. Cowan, E. Feigl, C. Grupen, J. Minguet-Rodriguez, F. Rivera, P. Saraiva, L. Smolik, F. Stephan

Fachbereich Physik, Universität Siegen, 57068 Siegen, Fed. Rep. of Germany ${ }^{16}$

M. Apollonio, L. Bosisio, R. Della Marina, G. Giannini, B. Gobbo, G. Musolino

Dipartimento di Fisica, Università di Trieste e INFN Sezione di Trieste, 34127 Trieste, Italy

J. Rothberg, S. Wasserbaech

Experimental Elementary Particle Physics, University of Washington, WA 98195 Seattle, U.S.A.

S.R. Armstrong, P. Elmer, Z. Feng, ${ }^{27}$ D.P.S. Ferguson, Y.S. Gao, ${ }^{23}$ S. González, J. Grahl, T.C. Greening, O.J. Hayes, H. Hu, P.A. McNamara III, J.M. Nachtman, W. Orejudos, Y.B. Pan, Y. Saadi, I.J. Scott, J. Walsh, Sau Lan Wu, X. Wu, J.M. Yamartino, M. Zheng, G. Zobernig

Department of Physics, University of Wisconsin, Madison, WI 53706, USA ${ }^{11}$

\footnotetext{
${ }^{1}$ Now at CERN, 1211 Geneva 23, Switzerland.

${ }^{2}$ Supported by Dirección General de Investigación Científica y Técnica, Spain.

${ }^{3}$ Also at Dipartimento di Fisica, INFN, Sezione di Catania, Catania, Italy.

${ }^{4}$ Also Istituto di Fisica Generale, Università di Torino, Torino, Italy.

${ }^{5}$ Also Istituto di Cosmo-Geofisica del C.N.R., Torino, Italy.

${ }^{6}$ Supported by the Commission of the European Communities, contract ERBCHBICT941234.

${ }^{7}$ Supported by CICYT, Spain.

${ }^{8}$ Supported by the National Science Foundation of China.

${ }^{9}$ Supported by the Danish Natural Science Research Council.

${ }^{10}$ Supported by the UK Particle Physics and Astronomy Research Council.

${ }^{11}$ Supported by the US Department of Energy, grant DE-FG0295-ER40896.

${ }^{12}$ Now at Dragon Systems, Newton, MA 02160, U.S.A.

${ }^{13}$ Supported by the US Department of Energy, contract DE-FG05-92ER40742.

${ }^{14}$ Supported by the US Department of Energy, contract DE-FC05-85ER250000.

${ }^{15}$ Permanent address: Universitat de Barcelona, 08208 Barcelona, Spain.

${ }^{16}$ Supported by the Bundesministerium für Bildung, Wissenschaft, Forschung und Technologie, Fed. Rep. of Germany.

${ }^{17}$ Supported by the Direction des Sciences de la Matière, C.E.A.

${ }^{18}$ Supported by Fonds zur Förderung der wissenschaftlichen Forschung, Austria.

${ }^{19}$ Permanent address: Kangnung National University, Kangnung, Korea.

${ }^{20}$ Now at DESY, Hamburg, Germany.

${ }^{21}$ Also at CERN, 1211 Geneva 23, Switzerland.

${ }^{22}$ Supported by the US Department of Energy, grant DE-FG03-92ER40689.

${ }^{23}$ Now at Harvard University, Cambridge, MA 02138 , U.S.A.

${ }^{24}$ Now at Max-Plank-Institüt für Kernphysik, Heidelberg, Germany.

${ }^{25}$ Also at Istituto di Matematica e Fisica, Università di Sassari, Sassari, Italy.

${ }^{26}$ Permanent address: Sung Kyun Kwan University, Suwon, Korea.

${ }^{27}$ Now at The Johns Hopkins University, Baltimore, MD 21218, U.S.A.
} 


\section{Contents}

1 Introduction $\quad 1$

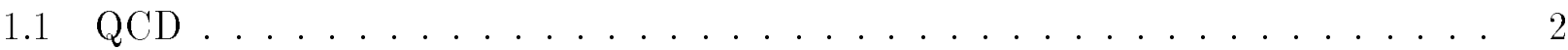

1.1.1 QCD Lagrangian and Fundamental Properties . . . . . . . . . . 2

1.1.2 The Process $e^{+} e^{-} \rightarrow$ hadrons ................. 5

1.2 The ALEPH Detector . . . . . . . . . . . . . 7

1.2.1 Particle tracking . . . . . . . . . . . . . 7

1.2.2 Specific Ionization Measurement . . . . . . . . . . . . . 9

1.2 .3 Calorimetry . . . . . . . . . . . . . . . . 9

1.2.4 The Trigger System . . . . . . . . . . . . . . . 10

1.2.5 The Identification of $K^{0}$ mesons and $\Lambda$ Hyperons . . . . . . . . . . 10

1.2.6 Energy Flow Determination ................. 11

1.2.7 Heavy Quark Tagging ..................... 11

1.3 Data Analysis Overview . . . . . . . . . . . . . . 12

1.3.1 Track and Event Selection . . . . . . . . . . . . 12

1.3.2 Corrections for Detector Effects . . . . . . . . . . . . 13

2 Global Event Structure and Tuning of Model Parameters $\quad 15$

2.1 Definition of Observables . . . . . . . . . . . . . 15

2.2 Analysis Technique and Results . . . . . . . . . . . . . 17



2.3.1 Description of the Models . . . . . . . . . . . . . 26

2.3.2 Fitting of Model Parameters . . . . . . . . . . . . 28

2.3.3 Discussion of the Results . . . . . . . . . . . . . . 30

3 Hard QCD $\quad 34$

3.1 Parton Spins. . . . . . . . . . . . . . . . . . . . 34

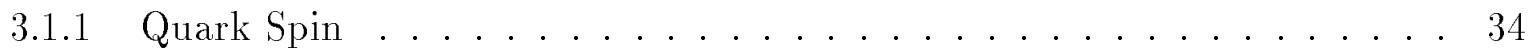



3.2 Measurements of the Strong Coupling Constant . . . . . . . . . . . . . 37

3.2.1 Z Hadronic Width . . . . . . . . . . . . . . . . 39

3.2.2 The Hadronic Width of the Tau . . . . . . . . . . . . . . 40

3.2.3 Event Shapes and Jet Rates . . . . . . . . . . . . . . . . 44

3.2.4 Scaling Violations in Fragmentation Functions . . . . . . . . . . . 50

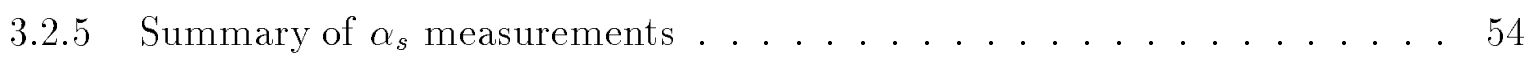

3.2.6 The Running of $\alpha_{s} \ldots \ldots \ldots \ldots \ldots$. . . . . . . . . . . . . . . . . . .

3.3 Angular Dependence of Event Shapes . . . . . . . . . . . . . . . 56 
3.4 Test of the Flavour Independence of $\alpha_{s} \ldots \ldots \ldots \ldots$

3.5 Colour Factors of QCD . . . . . . . . . . . . . . . . . 61

3.5.1 Determination using four-jet events .............. 63

3.5.2 Determination using two- and three-jet events . . . . . . . . . 64

3.5.3 Information from the running of $\alpha_{s} \ldots \ldots \ldots \ldots$

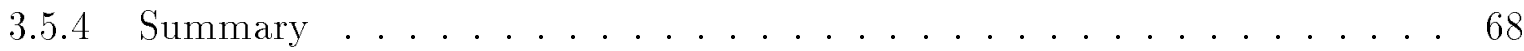

4 Semi-Soft QCD $r$

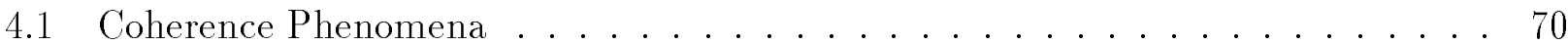

4.1.1 Inclusive Distribution of $-\ln x_{p} \ldots \ldots \ldots \ldots 71$

4.1.2 Energy Dependence of the Peak of the $-\ln x_{p}$ Distribution . . . . . . 74

4.1.3 Particle-Particle Correlations . . . . . . . . . . . . . . 77

4.1.4 Energy-Multiplicity-Multiplicity Correlations . . . . . . . . . . . 79

4.1.5 Particle Flow in Interjet Regions (String Effect) . . . . . . . . . . . 80

4.2 Charged Particle Multiplicities . . . . . . . . . . . . . . . 84

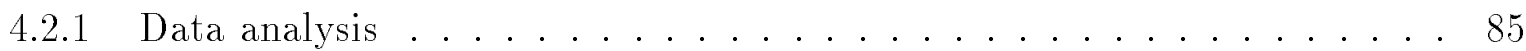

4.2.2 Model Independent Results . . . . . . . . . . . . . 86

4.2.3 Energy Dependence of the Charged Multiplicity Distribution . . . . . . 88

4.2.4 Charged Particle Multiplicities in Rapidity Windows . . . . . . . . . . 90

4.3 Intermittency . . . . . . . . . . . . . . . . . . 93

4.4 Subjet Structure of Hadronic Events . . . . . . . . . . . . . . . . 95

4.4.1 Subjet Structure of Two- and Three-Jet Events . . . . . . . . . . . 96

4.4.2 Subjet Structure of Identified Quark and Gluon Jets . . . . . . . . . 98

4.5 Properties of Tagged Jets in Symmetric Three-Jet Events . . . . . . . . . . . . 100

4.5.1 Data Analysis . . . . . . . . . . . . . . . 101

4.5.2 Unfolding of the Jet Properties . . . . . . . . . . . . 102

4.5.3 Measured Quark and Gluon Jet Properties . . . . . . . . . . . . 103

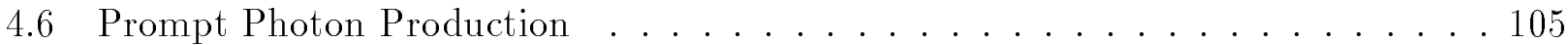

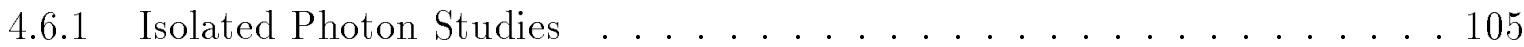

4.6 .2 "Democratic" Analysis . . . . . . . . . . . . . . 107

5 Hadronization $\quad 113$

5.1 Search for Free Quarks . . . . . . . . . . . . . . . . 113

5.2 Inclusive Production of Identified Hadrons . . . . . . . . . . . . . . . 114

5.2.1 Identified Stable Charged Particles . . . . . . . . . . . 114

5.2 .2 Single Photons . . . . . . . . . . . . . . 118

5.2 .3 Neutral Pions . . . . . . . . . . . . . . 120 
$5.2 .4 \eta$ and $\eta^{\prime}$ Mesons . . . . . . . . . . . . . . . . 122

5.2.5 Light Strange Particles . . . . . . . . . . . . . . . 124

5.2 .6 Heavy Strange Particles . . . . . . . . . . . . . . 126

5.2 .7 Neutral Vector Mesons . . . . . . . . . . . . . . . . 132



5.2 .9 Summary and Discussion . . . . . . . . . . . . . 137

5.3 Two-Particle Correlations . . . . . . . . . . . . . . 139

5.3.1 Proton-Antiproton Correlations . . . . . . . . . . . 140

5.3 .2 Strangeness Correlations . . . . . . . . . . . . . 142

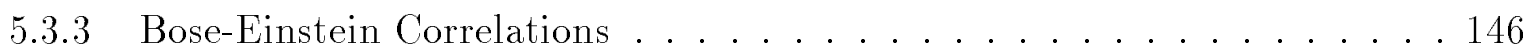

6 Summary 151

A $R_{l}$ and $R_{\tau}$ for Arbitrary Colour Factors 153

A.1 The Running Coupling Constant and Masses . . . . . . . . . . . . . 153

A.2 Theoretical Predictions for $R_{\gamma} \ldots \ldots \ldots \ldots$. . . . . . . . 154

A.3 The Theoretical Prediction for $R_{l} \ldots \ldots \ldots$. . . . . . . . 155

A.4 The Theoretical Prediction for $R_{\tau} \ldots \ldots \ldots \ldots 6$ 


\section{Introduction}

After the discovery of the partonic structure of hadrons which led to the quark-parton model [1], Quantum Chromodynamics (QCD) was formulated in analogy to Quantum Electrodynamics (QED) as a gauge theory which describes strong interactions between quarks via the exchange of massless gauge bosons, the gluons. Using the knowledge, obtained, for example, from hadron spectroscopy, the measurement of the $\pi^{0}$ decay rate or the total $e^{+} e^{-}$annihilation cross section into hadronic final states [2], that quarks have three internal degrees of freedom, it was natural to assume that those degrees of freedom are associated with the charge of QCD, called "colour". The additional requirement that bound states of three quarks or a quark-antiquark pair exist as colour singlets, i.e. without net colour charge, made SU(3) the natural candidate for the gauge group of QCD [3].

An important difference between QED and QCD is that the gauge bosons of QCD carry colour charge. Gluons thus couple directly to gluons. A consequence is that vacuum polarization effects produce an anti-screening of the bare QCD charges. This results in the strong coupling constant growing at large distances and becoming small at short distances [4, 5]. This could possibly explain why quarks are not observed as free particles [6] and at the same time renders perturbation theory applicable to describe processes involving large momentum transfers.

Study of the $\mathrm{Z}$ bosons produced in $e^{+} e^{-}$annihilations not only provides an ideal laboratory to study electro-weak interactions, but also permits precision measurements of strong interactions by studying QCD corrections to the well defined initial state of a Z decaying into a quark-antiquark pair. The LEP centre-of-mass energy of $E_{c m}=91.2 \mathrm{GeV}$ is about three times higher than at PEP/PETRA and about 50\% larger than at TRISTAN. Perturbative QCD predicts corrections which evolve as $1 / \ln E_{c m}[4,5]$ whereas non-perturbative effects are expected to scale with $1 / E_{c m}$ [7]. The higher energy thus improves the prospects for precision tests of perturbative QCD. As an added advantage the cross section at the energy corresponding to the $\mathrm{Z}$ resonance is much larger than for any of the machines mentioned above.

Since the startup of LEP several million hadronic Z decays have been collected and analyzed for precision measurements of the strong coupling constant, for tests of its flavour independence, to probe the gauge structure of QCD and to study coherence effects and the hadron formation mechanism. This paper summarizes the studies using the ALEPH detector in order to probe the structures of QCD at the hard perturbative level, the semi-soft level of leading-logarithm QCD and the hadronization stage.

The remainder of Section 1 is devoted to a summary of the main properties of QCD and of the QCD description of the $e^{+} e^{-}$annihilation into hadrons process and to the description of the ALEPH detector and a data analysis overview. This serves also to fix the notation and conventions. Section 2 deals with the measurement of global properties of hadronic events and its use in the determination of the free parameters of the hadronization models used for all analyses. The basic components of QCD are studied in Section 3. This includes the determination of the spin of quarks and gluons, measurements of the strong coupling constant and of the structure constants of the QCD gauge group. All these studies are based on predictions that are perturbative in nature, with relatively small non-perturbative corrections. Section 4 covers studies which probe lower $Q^{2}$ scales, for which hadronization effects can be important. The goal here is to understand something about the overlap region between perturbative and non-perturbative QCD. Coherence phenomena, charged particle multiplicities, subjet multiplicities, quark and gluon jet properties and prompt photon production are covered in this section. Finally, Section 5 includes studies of the hadronization phase itself, for which 
there are essentially no firm QCD predictions. The inclusive production of identified hadrons is studied here in detail, together with two-particle correlations. These kinds of studies could some day shed some light on the mechanism of confinement. Here, they are essentially used to study and compare the present hadronization models, and to fix some of their free parameters.

This paper includes summaries of previously published results as well as updates, with more statistics, of previously published analyses. There are also a number of analyses that are presented here for the first time:

- Determinations of the spin of quarks and gluons are presented in Sections 3.1.1 and 3.1.2, respectively.

- Oriented event shape distributions are studied in Section 3.3.

- A measurement of the QCD colour factors with two- and three-jet events is shown in Section 3.5.2. Another analysis using information from the running of $\alpha_{s}$ is shown in Section 3.5.3.

- Two studies of coherence phenomena using particle-particle correlations and energymultiplicity-multiplicity correlations are presented in Sections 4.1.3 and 4.1.4, respectively.

- The string effect is studied in Section 4.1.5

- Analyses of the inclusive production of identified single photons, neutral pions and strange hyperons are presented in Sections 5.2.3, 5.2.2 and 5.2.6.

- Proton-antiproton correlations are studied in Section 5.3.1.

\section{$1.1 \quad$ QCD}

In this section the basics of QCD and its application to the reaction $e^{+} e^{-} \rightarrow$ hadrons are briefly reviewed. This serves primarily to define notation and summarize the theoretical framework of the analyses.

\subsubsection{QCD Lagrangian and Fundamental Properties}

Strong interaction phenomena currently are best understood in the framework of QCD, which describes the interactions of spin-1/2 quarks and spin-1 gluons (collectively called partons). The quarks are described by Dirac fields $q$ which come in one of six flavours, $q=u, d, s, c, b, t$. Quarks were first introduced by Gell-Mann [8] and Zweig [9] in 1964 to describe the spectrum of observed hadrons. Several years later, experiments on deep inelastic electron-nucleon scattering provided evidence that nucleons are composed of point-like constituents, which were subsequently identified with quarks $[10,11]$.

In addition to flavour, the quarks $q$ are characterized by the quantum number colour, i.e. $q_{a}$ with $a=1, \ldots, N_{c}$. The number of colours $N_{c}$ in QCD must be at least three to construct a totally asymmetric wave function for the $\Delta^{++}$baryon, which consists of three $u$ quarks. Measurements of the $\pi^{0}$ lifetime and the total cross section for $e^{+} e^{-} \rightarrow$ hadrons lead to $N_{c}=3$. The concepts of quarks and colour were ultimately merged into a gauge theory of strong interactions based on the gauge group $\mathrm{SU}(3)$. (The historical development of QCD is described, e.g., in [12].) 
The Lagrangian of QCD is constructed along similar lines to that of QED. It is given by (see, e.g., [13])

$$
\mathcal{L}=\sum_{q=u, d, \ldots} \bar{q}_{a}\left(i \gamma^{\mu} D_{\mu}-m_{q}\right)_{a b} q_{b}-\frac{1}{4} F_{\mu \nu}^{A} F^{A \mu \nu},
$$

where the covariant derivative is

$$
\left(D_{\mu}\right)_{a b}=\delta_{a b} \cdot \partial_{\mu}+i g_{s} t_{a b}^{A} G_{\mu}^{A}
$$

and the field strength tensor is

$$
F_{\mu \nu}^{A}=\partial_{\mu} G_{\nu}^{A}-\partial_{\nu} G_{\mu}^{A}-g_{s} f^{A B C} G_{\mu}^{B} G_{\nu}^{C} .
$$

Here the gauge particles of the theory, called gluons, are represented by vector fields $G_{\mu}^{A}$, where $A=1, \ldots, 8$. It is understood here that repeated indices are summed $(0,1,2,3$ for Lorentz indices $\mu, \nu ; 1,2,3$ for the colour indices $a$ and $b ; 1, \ldots, 8$ for the indices $A, B, C)$. The $3 \times 3$ matrices $t^{A}$ are the generators of the group SU(3) (see, e.g., [14]). They satisfy the commutation relations

$$
\left[t^{A}, t^{B}\right]=i f^{A B C} t^{C}
$$

where $f^{A B C}$ are the structure constants of SU(3). The coupling of the quark and gluon fields is given in Eq. (1) by the coupling strength $g_{s}$ or equivalently

$$
\alpha_{s}=\frac{g_{s}^{2}}{4 \pi}
$$

A guiding principle in determining the form of the Lagrangian (1) is that it should remain invariant under a local SU(3) gauge transformation:

$$
\begin{aligned}
q_{a} & \rightarrow U_{a b} q_{b} \\
\bar{q}_{a} & \rightarrow U_{a b}^{*} \bar{q}_{b} \\
G_{\mu}^{A} & \rightarrow G_{\mu}^{A}+\partial_{\mu} \omega(x)+g_{s} f^{A B C} \omega^{B}(x) G_{\mu}^{C},
\end{aligned}
$$

where the $3 \times 3$ matrix $U$ is

$$
U=\exp \left(-i g_{s} \omega^{A}(x) t^{A}\right)
$$

and $\omega^{A}(x)(A=1, \ldots 8)$ are arbitrary real quantities which depend in general on the space-time coordinates $x=(t, \vec{x})$. A gluon mass term of the form $m_{g}^{2} G_{\mu}^{A} G^{\nu A}$ would violate gauge invariance and hence is not allowed.

The QCD Lagrangian (1) leads to the three elementary vertices shown in Fig. 1. Amplitudes for various processes involving quarks and gluons can be obtained using the Feynman rules derivable from the QCD Lagrangian (see, e.g., [13]). The amplitudes for $q \bar{q} g$ and $g g g$ in Fig. 1 (a) and (b) are proportional to the coupling $g_{s}$, whereas the four-gluon vertex (c) is proportional to $g_{s}^{2}$.

Sums over possible colour combinations for final state partons lead to the following colour factors:

$$
\begin{array}{ccc}
\operatorname{Tr} t^{A} t^{B}=T_{F} \delta^{A B} & \rightarrow & T_{F}=1 / 2 \\
t_{a b}^{A} t_{b c}^{A}=C_{F} \delta_{a c} & \rightarrow & C_{F}=\left(N_{c}^{2}-1\right) /\left(2 N_{c}\right)=4 / 3 . \\
f^{A B C} f^{A B D}=C_{A} \delta^{C D} & \rightarrow & C_{A}=N_{c}=3
\end{array}
$$




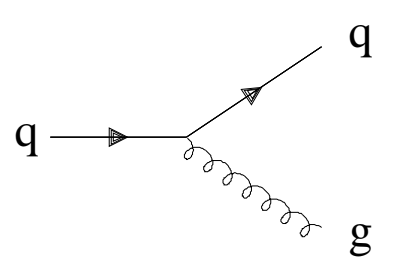

(a)

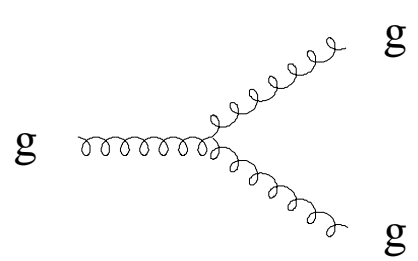

(b)

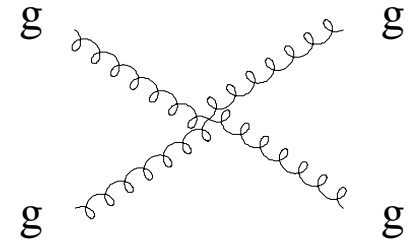

(c)

Figure 1: Elementary vertices of QCD: (a) quark-gluon vertex, (b) triple gluon vertex, (c) four-gluon vertex.

These relations hold for a general colour gauge theory with gauge group $\mathrm{SU}\left(N_{c}\right)$; the numerical values given are for $N_{c}=3$. The colour factor $C_{F}$ is proportional to the probability for the branching $q \rightarrow q g, C_{A}$ gives the corresponding value for $g \rightarrow g g$ and $T_{F}$ for $g \rightarrow q \bar{q}$.

The amplitudes corresponding to the graphs shown in Fig. 2 are ultraviolet divergent. Renormalization leads to a running coupling $\alpha_{s}\left(\mu^{2}\right)$ where $\mu^{2}$ is the renormalization scale. The dependence of $\alpha_{s}$ on $\mu^{2}$ is given by the renormalization group equation

$$
\begin{aligned}
\mu^{2} \frac{\partial \alpha_{s}}{\partial \mu^{2}} & =\beta\left(\alpha_{s}\right) \\
& =-b_{0} \alpha_{s}^{2}+b_{1} \alpha_{s}^{3}+O\left(\alpha_{s}^{4}\right) .
\end{aligned}
$$

The right-hand side of (10) is the beta function of QCD. The values of the coefficients $b_{0}, b_{1}, \ldots$ depend on the renormalization scheme used; all formulae in this paper use the $\overline{\mathrm{MS}}$ scheme (see, e.g., [13]). The first two coefficients are, in fact, scheme independent and have been computed to be

$$
b_{0}=\frac{11 C_{A}-2 n_{f}}{12 \pi}, b_{1}=\frac{17 C_{A}^{2}-5 C_{A} n_{f}-3 C_{F} n_{f}}{24 \pi^{2}} .
$$

Here $n_{f}$ is the number of active flavours, i.e. flavours with $m_{q}$ sufficiently small compared to the energy scale of the process that they contribute to quark loop corrections of the type shown in Fig. 2(a). These corrections give a positive contribution to the beta function. The gluon loops (Fig. 2(b)), however, yield a negative contribution, and the total beta function is negative as long as the number of active flavours $n_{f}$ is sufficiently small to satisfy $11 C_{A}-2 n_{f}>0$, i.e. $n_{f}<33 / 2$. With the six known quark flavours this criterion is met, and at the experimental energies used here $\left(E_{c m} \approx M_{Z}\right)$, the top quark does not contribute significantly, so one has $n_{f}=5$. The fact that the beta function is negative leads to a decrease in $\alpha_{s}$ for increasing values of the scale, which is known as asymptotic freedom. It is this property of QCD, discovered in 1973 by Gross and Wilczek [4] and Politzer [5], that allows reliable predictions from perturbation theory for processes involving high momentum transfers. This is in contrast to the situation in QED, where a positive beta function leads to a higher coupling strength (effective electric charge) as the energy scale of the process increases.

The renormalization group equation can be solved to relate $\alpha_{s}$ at one scale $\mu^{2}$ to that at another scale $Q^{2}$. To second order, and including the resummation of leading logarithms, this gives

$$
\alpha_{s}\left(Q^{2}\right)=\frac{\alpha_{s}\left(\mu^{2}\right)}{w}\left(1-\frac{b_{1}}{b_{0}} \frac{\alpha_{s}\left(\mu^{2}\right)}{w} \ln w\right)
$$

where

$$
w=1-b_{0} \alpha_{s}\left(\mu^{2}\right) \ln \frac{\mu^{2}}{Q^{2}}
$$




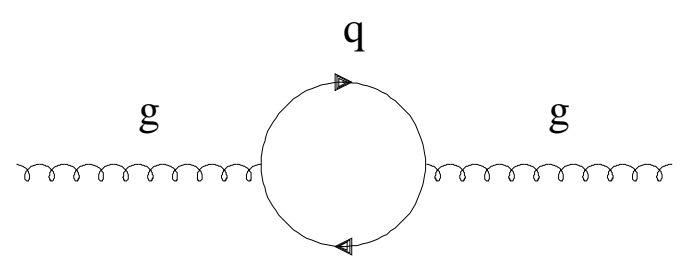

(a)



(b)

Figure 2: Virtual corrections to the gluon propagator: (a) quark loop, (b) gluon loop.

\subsubsection{The Process $e^{+} e^{-} \rightarrow$ hadrons}

The reaction $e^{+} e^{-} \rightarrow$ hadrons can be viewed as proceeding through the four phases shown in Fig. 3.

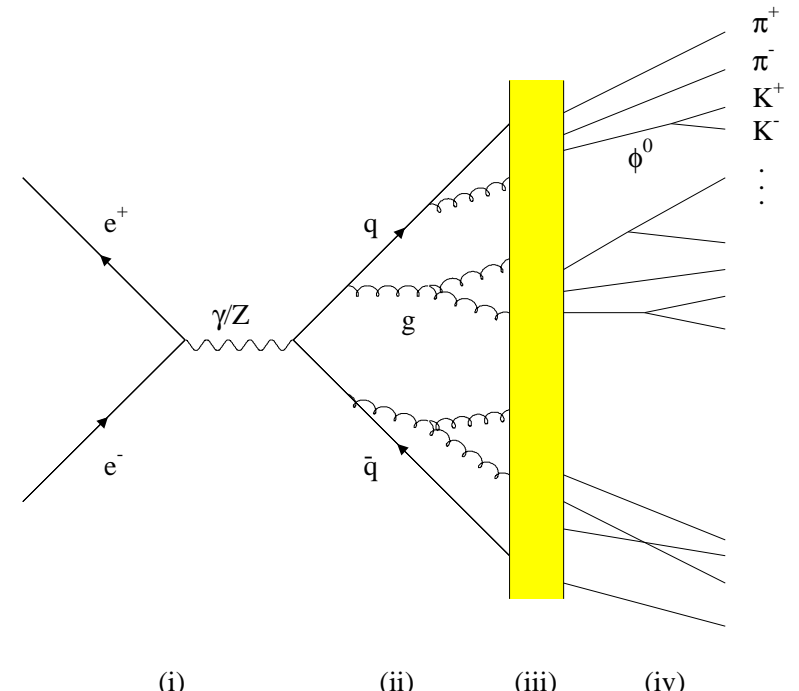

(i)

(ii) (iii)

(iv)

Initially, the Z-resonance decays into a highly virtual quark-antiquark pair. This stage, along with possible photon radiation, is well described by the Standard Model of electroweak interactions. The differential cross section for production of a quark-antiquark pair is then given by

$$
\frac{d \sigma}{d \cos \theta}=\sigma_{q \bar{q}} \frac{3}{8}\left(1+\frac{8}{3} A_{F B} \cos \theta+\cos ^{2} \theta\right)
$$

where $\sigma_{q \bar{q}}$ is the total cross section and $\theta$ is the angle of the outgoing quark with respect to the incoming electron direction. The forward-backward asymmetry $A_{F B}$ arises from the interplay of vector and axial-vector couplings, and is small for $E_{c m}=M_{Z}$. Since in the studies presented here no attempt has been made to separate quarks from antiquarks, the term in (14) proportional to $\cos \theta$ becomes irrelevant.

At centre of mass energies near the $Z$ resonance the contribution from annihilation into a photon can be neglected. The total cross section for production of a $q \bar{q}$ pair is then given at Born level by

$$
\sigma_{q \bar{q}}=\frac{12 \pi \Gamma_{e e} \Gamma_{q \bar{q}}}{M_{Z}^{2} \Gamma_{Z}^{2}},
$$


where $M_{Z}$ is the mass of the $Z$ boson, and $\Gamma_{e e}, \Gamma_{q \bar{q}}$, and $\Gamma_{Z}$ are the partial widths for $Z$ decay into $e^{+} e^{-}, q \bar{q}$ and the total width, respectively. Summing over the kinematically accessible quark flavours $q=u, d, s, c, b$ and taking account of higher order corrections, such as initial state photon radiation, results in a total hadronic cross section of around $30 \mathrm{nb}$. It is this large cross section that makes possible the high statistics measurements presented here.

Final states having more partons in addition to the primary quark-antiquark pair can be described by QCD using perturbation theory. To first order in $\alpha_{s}$, the differential cross section for $e^{+} e^{-} \rightarrow q \bar{q} g$ is given by [15]

$$
\frac{d^{2} \sigma}{d x_{1} d x_{2}}=\sigma_{q \bar{q}} \frac{C_{F} \alpha_{s}}{2 \pi} \frac{x_{1}^{2}+x_{2}^{2}}{\left(1-x_{1}\right)\left(1-x_{2}\right)},
$$

where

$$
x_{i}=2 E_{i} / E_{c m}
$$

are the parton energies normalized to the maximum allowed energy $E_{\mathrm{cm}} / 2$ with $i=1,2,3$ $(=q, \bar{q}, g)$. Exact matrix elements have been computed only to second order in $\alpha_{s}$, and can be found in [16]. This describes a maximum of four partons in the final state, $q \bar{q} g g$ or $q \bar{q} q \bar{q}$.

For predictions of final states with larger numbers of partons, one can use the parton shower approach, based on the leading logarithm approximation. The main idea here is to reorganize the perturbative expansion so that the terms with leading collinear singularities are summed to all orders [17]. The result for the cross section can then be reinterpreted as a sequence of parton branchings $q \rightarrow q g, g \rightarrow g g$ and $g \rightarrow q \bar{q}$. The virtual mass $Q$ of the partons decreases after each decay, which leads to a corresponding increase in the strong coupling $\alpha_{s}\left(Q^{2}\right)$. The shower is terminated at some virtuality cut-off $Q_{0}$, below which $\alpha_{s}$ becomes so large that perturbation theory is no longer valid. At this point, other calculation techniques (e.g. phenomenological models) must be invoked to describe the production of final state hadrons.

In order to include leading infrared singularities (where the energy of the emitted gluon vanishes) one must account for the effects of soft gluon interference. It has been shown that the effect of this interference is completely destructive to leading order outside of an angle-ordered region for each parton decay [18]. That is, one can preserve the probabilistic interpretation of the cascade simply by restricting the phase space allowed for each parton branching such that the opening angles always decrease. The phase space constraint leads to a suppression of the number of soft partons. Quantities sensitive to angular ordering are investigated in Section 4.1.

In principle QCD should be able to provide a complete description of hadronic final states, including the transformation of partons into colour neutral hadrons (hadronization). At present, however, this task is computationally impossible, since the low momentum-transfer (or virtual mass) scale $Q_{0}$ involved in hadronization leads to a coupling constant $\alpha_{s}\left(Q_{0}^{2}\right)$ too large to allow meaningful predictions from perturbation theory. In place of QCD predictions for hadronization one needs to introduce phenomenological models. If both the perturbative description and the models were perfect, the value of $Q_{0}$ would be arbitrary. In practice, $Q_{0}$ is adjusted to achieve the best overall description of the data; typical values are in the range $0.6-1.6 \mathrm{GeV}$, corresponding to $\alpha_{s}\left(Q_{0}^{2}\right) \approx 0.3-0.5$ (cf. Section 2.3 ).

Several approaches to the hadronization stage have been developed and implemented as Monte Carlo event generators. These programs begin by generating an initial quark-antiquark pair (possibly accompanied by initial state photon radiation) using the differential cross section 
given by the electroweak Standard Model. The evolution of the parton system is treated with some implementation of the perturbative techniques mentioned above, i.e. fixed-order matrix elements or parton showers. The resulting system of partons will be referred to in the following as the "parton level" of an event generator.

Models for relating the parton and hadron levels involve phenomenological constructs such as clusters and strings. Although the hadron production mechanisms can be quite different in different models, they possess important common features, e.g. local conservation of quantum numbers such as charge, flavour, and baryon number. A more detailed description of hadronization models is given in Section 2.3.1.

\subsection{The ALEPH Detector}

The ALEPH detector operating at the CERN LEP electron-positron collider is designed to study a wide range of phenomena produced in electron-positron collisions at centre of mass energies up to $200 \mathrm{GeV}$. The results presented in this paper have been obtained at the $\mathrm{Z}$ resonance where the events produced from $Z$ decays can be complex, with particles distributed over $4 \pi$ in solid angle. In the case of hadronic decays the events can have high multiplicity with around twenty charged particles and a similar number of neutrals. The detector thus was designed to have high three-dimensional granularity, hermetic coverage and accurate vertexing. The detector and its performance have been described in detail elsewhere [19, 20]; an overview, containing only essential information, is presented in this section. The detector is constructed from independent modular subdetectors, arranged as a cylindrical "barrel" section closed by

two "endcaps". The overall dimensions of the detector are approximately $12 \times 12 \times 12 \mathrm{~m}^{3}$ and its weight is about 3000 tonnes. A schematic diagram is given in Fig. 4.

Because of the creation of $e^{+} e^{-}$pairs from photons, the loss of energy by electrons and multiple Coulomb scattering, it is important that the material traversed by radiation from the interaction point is kept to a minimum. The ALEPH beam pipe, which holds the LEP machine vacuum, is a thin $5.5 \mathrm{~m}$ long tube which traverses the detector. The tube has an inner diameter of $106 \mathrm{~mm}$ and is made from $1.1 \mathrm{~mm}$ thick beryllium in the $760 \mathrm{~mm}$ long central region. The thickness of materials traversed by particles in passing through the detector is a function of the polar angle; for angles greater than 40 degrees the total material, including the beam pipe, preceding the electromagnetic calorimeter is less than 0.25 radiation lengths.

The origin of the ALEPH coordinate system is the theoretical beam crossing point, the midpoint of the straight section between the two nearest LEP quadrupoles. The positive $x$-axis points towards a vertical line through the LEP centre and is horizontal by definition. The positive $z$ axis is along the nominal $e^{-}$beam direction. The $y$ direction is orthogonal to $x$ and $z$ and points upwards.

\subsubsection{Particle tracking}

The tracking system involves three detectors: a silicon vertex detector, a conventional drift chamber and a large time projection chamber. These are contained within a $1.5 \mathrm{~T}$ magnetic field, produced by a superconducting magnet coil, in order to obtain accurate measurement of the momenta of charged particles.

The silicon vertex detector (VDET) [21] is used for particle tracking close to the electronpositron interaction point and vertex detector hits are used also to provide additional precision for tracks already reconstructed in the outer tracking detectors. The VDET consists of two 


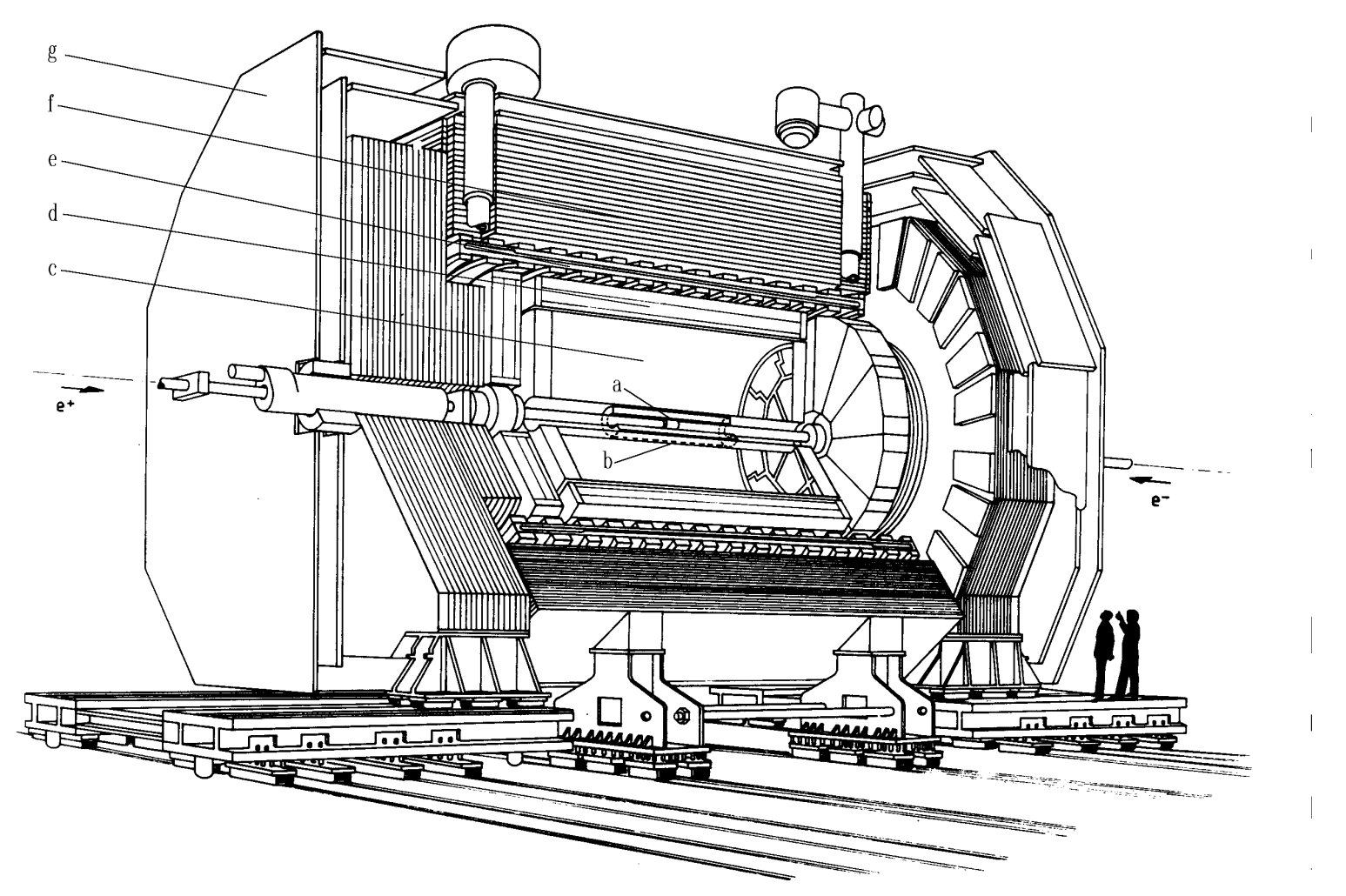

Figure 4: Schematic diagram of the ALEPH detector: (a) silicon vertex detector (VDET), (b) inner tracking chamber (ITC), (c) time projection chamber (TPC), (d) electromagnetic calorimeter (ECAL), (e) superconducting magnet coil, (f) hadron calorimeter (HCAL) and (g) muon chambers.

concentric layers of double-sided silicon microstrip detectors positioned at average radii of 6.5 $\mathrm{cm}$ and $11.3 \mathrm{~cm}$, covering respectively $85 \%$ and $69 \%$ of the solid angle. Each silicon wafer has readout both parallel $(z)$ and perpendicular $(r \phi)$ to the beam direction where $r$ is the radial distance from the origin and $\phi$ is the azimuthal angle. The spatial resolution given by the detector is $12 \mu \mathrm{m}$ for the $r \phi$ coordinate and between $11 \mu \mathrm{m}$ and $22 \mu \mathrm{m}$ for the $z$ coordinate, depending on the polar angle of the charged particle.

The vertex detector is surrounded by a conventional multilayer axial-wire cylindrical drift chamber, the inner tracking chamber (ITC) [22], which is $200 \mathrm{~cm}$ long and measures the $r \phi$ positions of tracks at 8 radii between 13 and $29 \mathrm{~cm}$. The average resolution in the $r \phi$ coordinate from this chamber is $150 \mu \mathrm{m}$. The position of tracks along the beam direction, the $z$ coordinate, is determined with a precision of a few $\mathrm{cm}$ by measuring the difference in arrival times of the signals at each end of the wires of the chamber.

The time projection chamber (TPC) [23] is the principal tracking chamber in the ALEPH detector. It is a three-dimensional imaging drift chamber with uniform electric and magnetic fields along its cylindrical axis. The chamber is divided into two halves by a central membrane which is held at a negative high voltage. Charged particles passing through the TPC gas cause ionization of the argon-methane mixture. The $z$ coordinates are obtained from the drift time of the electrons from the ionizing tracks travelling with constant drift velocity from the track to an end-plate. The $r \phi$ coordinates are derived by interpolating between signals on cathode pads at the ends of the chamber. The TPC has an inner radius of $31 \mathrm{~cm}$, an outer radius of $180 \mathrm{~cm}$ and a drift length of $220 \mathrm{~cm}$ for each half of the chamber. For a track which traverses the TPC 
up to 21 space coordinates and 338 samples of ionization energy loss can be determined. After all corrections to the data an azimuthal coordinate resolution in the TPC of up to $173 \mu \mathrm{m}$ in $r \phi$ and a longitudinal resolution in the $z$ direction of up to $740 \mu \mathrm{m}$ is obtained.

The track finding efficiency in the TPC has been studied using both Monte Carlo simulation and visual scanning of events. In hadronic $\mathrm{Z}$ events, $98.6 \%$ of tracks that cross at least four pad rows in the TPC are successfully reconstructed; the small inefficiency due to track overlaps and small gaps in the detector is reproduced to better than $10^{-3}$ by the simulation.

Using the combined information from the TPC, ITC and VDET a transverse momentum resolution of $\sigma\left(1 / p_{T}\right)=0.6 \times 10^{-3}(\mathrm{GeV} / \mathrm{c})^{-1}$ has been measured with $45 \mathrm{GeV}$ muons from $\mathrm{Z}$ decays. The impact parameter resolution of charged particles measured in hadronic decays can be parametrized as $\sigma(\delta)=25 \mu \mathrm{m}+95 \mu \mathrm{m} / p(\mathrm{GeV} / \mathrm{c})^{-1}$ in both the $r \phi$ and $r z$ views. As discussed later, an important use of the precision impact parameter measurement is to detect b hadrons via their lifetime.

\subsubsection{Specific Ionization Measurement}

In addition to its role as a tracking device, the TPC serves to separate particle species according to measurements of their specific energy loss by ionization, $d E / d x$. The data from the TPC sense wires, located in the end-plates, are used for the $d E / d x$ measurement. Tracks must be at least $3 \mathrm{~cm}$ apart in $z$ in order to be resolved for $d E / d x$ purposes. For tracks which have at least $50 d E / d x$ measurements it is found that $d E / d x$ is very effective for electron identification, with greater than $3 \sigma$ separation up to a momentum of about $8 \mathrm{GeV} / \mathrm{c}$. In the relativistic rise region, the region of most interest in the ALEPH experiment, the pion-kaon separation is roughly constant at about $2 \sigma$, while the kaon-proton separation is about $1 \sigma$. Therefore, kaon and proton identification can be accomplished only on a statistical basis but, nonetheless, is an important means of reducing combinatorial background in many analyses.

\subsubsection{Calorimetry}

The electromagnetic calorimeter (ECAL) consists of a "barrel" surrounding the TPC and closed at each end by an "end-cap". It lies inside the superconducting magnet coil to minimize the amount of material preceding it and covers $98 \%$ of the full solid angle. The barrel and two endcaps each comprise 12 modules constructed from 45 layers of lead interleaved with proportional wire chambers. The energy and position of each shower is read out using small cathode pads with dimensions of around $30 \mathrm{~mm} \times 30 \mathrm{~mm}$. The cathode pads in each layer of the wire chambers are connected internally to form "towers" oriented towards the interaction point. Each tower is read out in three sections in depth of four, nine and nine radiation lengths. There are some 74,000 towers in all, each with an angular width of about $0.9^{\circ} \times 0.9^{\circ}$. The high granularity of the pads leads to excellent identification of electrons and photons within jets. The wire signals have very low noise and are used in an energy trigger which can work at a threshold as low as $200 \mathrm{MeV}$.

In order to reduce the sensitivity of the photon energy measurement to hadronic background and clustering effects, the energy is estimated from the signals in the four central towers of an electromagnetic cluster. The energy resolution for electrons in the energy range from 1 to 45

$\mathrm{GeV}$ is $\sigma(E) / E=(0.18 / \sqrt{E / \mathrm{GeV}}+0.009)$. The angular resolution for electromagnetic showers is approximately $\sigma(\theta, \phi)=(2.5 / \sqrt{E / G e V}+0.25) \operatorname{mrad}$. 
The hadron calorimeter (HCAL) is used together with the electromagnetic calorimeter to measure hadronic energy deposits and is also part of the muon identification system. It consists of 23 layers of limited streamer tubes $9 \times 9 \mathrm{~mm}^{2}$ in cross-section between layers of iron absorber each $50 \mathrm{~mm}$ thick, giving a total of 7.2 interaction lengths at $90^{\circ}$. The iron provides the flux return for the magnetic field and also serves as a muon filter. The calorimeter has a tower readout similar to the ECAL with pads of angular size about $3.7^{\circ} \times 3.7^{\circ}$ and is read out capacitively in 4788 projective towers. Digital readout from aluminum strips running the whole length of each tube provides a two-dimensional view of the development of hadronic showers. The pad and strip readout again provide important redundancy in energy measurements. The wire signals are used for triggering. The energy resolution of the calorimeter is $0.85 / \sqrt{E / G e V}$ for hadronic showers. The whole of HCAL is rotated by about $2^{\circ}$ relative to ECAL to avoid overlapping of the small gaps ("cracks") between modules.

Luminosity measurements are obtained with a precision of better than $0.1 \%$ via three complementary calorimeters positioned close to the beam pipe at a distance of approximately $260 \mathrm{~cm}$ from, and on both sides of, the interaction point. These calorimeters also improve the hermeticity of the detector by providing solid angle coverage down to 24 mrad from the beam axis.

Outside the iron are two double layers of streamer tubes, the muon chambers, which provide two space coordinates for particles leaving the detector.

\subsubsection{The Trigger System}

The ALEPH triggering system is organized into three levels. Level one decides whether or not to read out all detector elements. The level two trigger simply seeks to verify a level one charged track trigger by replacing the ITC tracking information with the more accurate TPC tracking information available $50 \mu$ s after the beam crossing. A level three software trigger is used to reject background such as beam-gas interactions and off-momentum particles hitting the vacuum chamber or collimators.

Hadronic $\mathrm{Z}$ decays are collected using a level one trigger in which deposits in the electromagnetic calorimeter (total-energy trigger) have an energy greater than $6 \mathrm{GeV}$ in the barrel or $3 \mathrm{GeV}$ in either endcap or greater than $1.5 \mathrm{GeV}$ in both endcaps in coincidence. An independent trigger requires that track segments in the drift chamber coincide with hits in a module of the hadron calorimeter, so requiring a certain penetration depth (muon-track trigger). This trigger is sensitive to muons and, with lower efficiency, to hadrons. Asking for either of these trigger requirements to be satisfied provides an efficiency of greater than $99.9 \%$ for selected hadronic decays.

\subsubsection{The Identification of $K^{0}$ mesons and $\Lambda$ Hyperons}

Charged particle tracks which do not originate from the main interaction point can arise from neutral particles, such as $K^{0}$ 's and $\Lambda$ 's, which decay inside the tracking volume to produce a characteristic $V^{0}$ signature.

An algorithm to identify $V^{0} \mathrm{~s}$ considers all pairs of oppositely charged particle tracks with momentum larger than $150 \mathrm{MeV} / \mathrm{c}$ and with more than five TPC coordinates and tests for the hypothesis that they originate from a common secondary vertex. The parameters of the fit are the track momenta and the coordinates of the secondary vertex. In order to ensure a separation between primary and secondary vertices, the proper lifetime of a given $V^{0}$ hypothesis is required 
to be between 0.2 and 5 times the expected lifetime. Since combinatorial background peaks strongly at forward decay angles, the cosine of the decay angle is required to be less than 0.85 for $K^{0} \mathrm{~s}$ and 0.95 for $\Lambda \mathrm{s}$. Furthermore, the distance of closest approach from the $V^{0}$ direction to the primary vertex is required to be less than $1.0 \mathrm{~cm}$ in the transverse plane. When a useful measurement of the specific ionization $(d E / d x)$ is available for a given track of a $V^{0}$ candidate it is required to be within three standard deviations of the expected value. Finally, a significant reduction in background is obtained by requiring a successful kinematical $V^{0}$ fit, constrained by the mass hypothesis and the primary vertex.

\subsubsection{Energy Flow Determination}

The simplest way to determine the total energy of an event recorded in the ALEPH detector is to sum the raw energy found in all calorimetric cells without performing any particle identification. This method yields a resolution of $\sigma(E) / E=1.2 / \sqrt{E / \mathrm{GeV}}$ for hadronic decays of the Z. In order to improve the resolution, an energy flow reconstruction algorithm has been developed making use of track momenta and taking advantage of the photon, electron and muon identification capabilities of the detector. In a first stage, charged particle tracks and calorimeter clusters are subjected to a sequence of "cleaning" operations, using the information from the tracking detectors and taking advantage of the redundancy in the readout of both calorimeters. The "cleaned" charged particle tracks are extrapolated to the calorimeters and groups of topologically connected tracks and clusters, called "calorimeter objects", are formed. Charged-particle tracks identified as electrons or muons and neutral electromagnetic energy objects identified as photons are removed from the "calorimetric objects". All the particles remaining in the calorimeter objects should be charged or neutral hadrons. The charged hadron energy in a given object can be determined from the tracking information. The excess calorimetric energy is assigned to neutral hadrons. A direct identification of neutral hadrons has not been attempted. The result of the above procedure is a set of "energy flow objects", or particles characterized by their energy and momenta. The energy resolution is reproduced by $\sigma(E)=(0.59 \pm 0.03) \sqrt{E / \mathrm{GeV}}+(0.6 \pm 0.3) \mathrm{GeV}$.

\subsubsection{Heavy Quark Tagging}

A large number of boosted $b$ hadrons are produced at the $\mathrm{Z}$ resonance in the ALEPH experiment. A high purity sample of $b$ hadrons can be obtained by identifying their semi-leptonic decay modes. However, this approach yields relatively low statistics when the semi-leptonic branching ratio is combined with the lepton identification efficiency. A large increase in statistics can be gained by using the fact that the long lifetime and large mass of $b$ hadrons give their decay products large impact parameters, defined as the distance of closest approach between a track and the $\mathrm{b}$ production point. The $b$ hadrons produced in $\mathrm{Z}$ decays travel typically several $\mathrm{mm}$ before decaying into several charged particles, including the decay products of secondary charmed hadrons. The masses of the final decay products are an order of magnitude less than those of the $b$ hadrons, resulting in highly energetic decays. Thus $b \bar{b}$ events are characterized by the presence of many charged tracks with significant impact parameters with respect to the $\mathrm{Z}$ decay point. Charmed hadrons have similar decay lengths but are lighter and their decays have lower charged multiplicities. The precise three-dimensional tracking information available from the VDET then can be exploited to provide accurate impact parameter measurements and thus allow a separation of $b$ 's from other hadrons. The typical primary vertex resolution is 
about $50 \mu \mathrm{m}$ for the horizontal coordinate, $10 \mu \mathrm{m}$ for the vertical and $60 \mu \mathrm{m}$ along the beam direction. The impact parameter resolution is about $70 \mu \mathrm{m}$.

The tracks in an event are clustered into jets, the jet definition having been optimized to reproduce the directions of $b$ hadrons within $b \bar{b}$ events. The $b$ production point is reconstructed for each event by combining the beam spot position with the track information for the particular event. The tracks are associated to their nearest jet and they are projected onto the plane perpendicular to this jet. This projection removes the bias due to tracks coming from secondary vertices, in the approximation that the jet axis reproduces the direction of the decaying particle. The projected tracks are then combined with the beam spot position to find the primary vertex.

The sensitivity to lifetime is increased by determining a sign for each three-dimensional impact parameter using the jet direction. The sign is positive if the point of closest approach between the track and the $b$ direction is in the same hemisphere as the track, the hemisphere being defined by the plane perpendicular to the $b$ direction and containing the $b$ production point.

By combining the impact parameter information from all tracks within an event, a tag variable can be constructed which can be used to distinguish $b \bar{b}$ events from those of lighter quarks. The efficiency for tagging $b$ 's is correlated to the purity of the $b$ sample. Within the angular acceptance of the VDET, a tagging efficiency in excess of $60 \%$ is achieved for a typical $b$ purity of $80 \%$.

\subsection{Data Analysis Overview}

In this section the general framework of the analyses will be described, including the selection of tracks, photons and other reconstructed objects, event selection, and the correction of measured quantities for various detector related effects. The exact procedures vary somewhat from one analysis to the next, and the differences from the general methods outlined here will be explained as required for each individual case.

\subsubsection{Track and Event Selection}

The event selection for most of the studies is based on tracks of charged particles. Tracks are selected that have at least four measured space coordinates from the TPC, a polar angle in the range $20^{\circ}<\theta<160^{\circ}$, and a transverse momentum with respect to the beam direction of $p_{\perp}>0.2 \mathrm{GeV}$. In addition, the closest radial distance of approach of the extrapolated track to the beam line, $d_{0}$, is required to be less than $2 \mathrm{~cm}$, and the $z$ coordinate of the point of closest radial approach, $z_{0}$, is required to be less than $5 \mathrm{~cm}$.

Using the selected tracks, the sphericity axis and the total charged energy $E_{c h}=\sum_{i} E_{i}=$ $\sum_{i} \sqrt{p_{i}^{2}+m_{\pi}^{2}}$ are computed. (The sphericity axis is described in Section 2.1.) Events are selected that have at least five charged tracks, $E_{c h}>15 \mathrm{GeV}$, and for which the polar angle of the sphericity axis is in the range $35^{\circ}<\theta_{s p h}<145^{\circ}$. The latter cut (not required for all analyses) ensures that the event is well contained within the detector. For data taken by the ALEPH detector between 1991 and 1994 these cuts result in approximately 2 million selected events. Most of the analyses presented use only a subset of these data. The largest background contribution is from events of the type $e^{+} e^{-} \rightarrow \tau^{+} \tau^{-}$, which are estimated to make up approximately $0.26 \%$ of the selected events. For most of the analyses considered, this can be neglected; in individual cases (e.g. the study of scaling violations in fragmentation functions) a background subtraction was carried out using a Monte Carlo model. 


\subsubsection{Corrections for Detector Effects}

Before a measured quantity can be compared with theoretical predictions or with the results of other measurements it must first be corrected for various detector related effects, such as geometrical acceptance, detector efficiency and resolution, decays, particle interactions with the material of the detector and the effects of event and track selection, and also for the effect of initial state photon radiation. For most of the analyses presented this is done with multiplicative correction factors $C$, relating the measured value of a quantity $X$, such as a bin content, to the corrected value,

$$
X_{\text {corrected }}=X_{\text {measured }} \cdot C \text {. }
$$

For distributions, the correction factors are computed individually for each bin. Several analyses, e.g. Sections 2, 4.2, involve a more sophisticated unfolding procedure; this is described in the corresponding sections.

The correction factors are computed according to the following procedure. First, hadronic events with the mixture of primary quark flavours as predicted by the Standard Model are generated using the program HVFL, which incorporates several components. The initial quarkantiquark pair and intial state photon radiation are generated with the program DYMU [24]. These are then passed to the Lund Parton Shower Model [25] (program JETSET version 7.3), in which the decay properties of heavy flavour ( $b$ and $c$ ) hadrons have been significantly extended.

The events are processed through the detector simulation program to produce simulated raw data, which are then processed by the same reconstruction and analysis programs as used for the real data. From these data the value of the observable in question is computed yielding $X_{M C+\text { det. sim. }}$.

A second set of Monte Carlo data is then generated, but here with initial state radiation turned off, and with all particles having mean lifetimes less than $10^{-9}$ seconds required to decay, and all other particles being treated as stable. In this way the charged particles defined to belong to the final state correspond approximately to those which are actually seen in the detector. For example, decay products of $K_{S}^{0}$ mesons and strange baryons are included as final state particles, whereas $K_{L}^{0}$ mesons are treated as stable. This second data set is used to compute the corresponding observable $X_{\text {generator }}$. The correction factor $C$ is the ratio of the two quantities,

$$
C=\frac{X_{\text {generator }}}{X_{M C+\text { det. sim }}} .
$$

Depending on the analysis, the quantity $X_{\text {generator }}$ may be computed using all particles, including neutrals (even if the measurement was only based on charged particles) or it may be computed using some well-defined subset of the particles, e.g. only charged. Application of the factors defined in this way results in measurements corrected to a well-defined particle composition and centre of mass energy without initial state radiation.

Although the correction factors are to a good approximation independent of the event generator used, a residual dependence remains and must be taken into account in the estimation of systematic errors. For example, in analyses where only charged particles are measured, but where the quantity $X_{\text {generator }}$ in (19) is computed using all particles (including neutrals), one relies on the Monte Carlo model to describe the effect of the neutrals on the observable. Another source of generator dependence arises from the smearing of distributions due to finite resolution. These effects are taken into account ("unfolded") by the technique described above, but the result is only correct to the extent that the distributions in nature are the same as those in the Monte Carlo model. One straightforward way of approaching this problem would be to compute 
the correction factors with several different Monte Carlo generators in order to estimate their model dependence. This is impractical, however, because of the large amount of computing time required by the detector simulation.

Most of the analyses use the following approximate technique to estimate the generator dependence of the correction factors. Instead of using data processed by the full detector simulation, a highly simplified simulation is performed by merely applying the same cuts on energy, transverse momentum, geometry, etc. as used for the real analysis. In some cases, finite energy resolution is simulated by smearing the generated energies according to the parameterized response of the detector. Simplified correction factors can then be computed as

$$
C_{\text {simplified }}=\frac{X_{\text {generator }}}{X_{M C+c u t s}}
$$

These factors can be computed for a variety of event generators, and the spread in the resulting values is taken as a contribution to the systematic uncertainty. For many quantities (especially distributions of event-shape variables) the simplified correction factors are qualitatively very similar to the factors based on the full detector simulation, indicating that the corrections are largely determined by cuts on geometry and energy, and/or by the correction for neutral particles if only charged particle information is used in the measurement.

The systematic errors due to model dependence of the correction factors are highly correlated from bin-to-bin. This is also the case for systematic uncertainties due to the modelling of the detector, which in most analyses are estimated by varying the experimental cuts in a certain range. In cases where the distribution is used to derive a further quantity (e.g. $\alpha_{s}$, Section 3.2), these correlations can be taken into account by correcting the distribution with different sets of simplified factors or by using different sets of cuts, obtaining the derived quantity from each alternative distribution, and then using the spread in the resulting values as a measure of its systematic error. 


\section{Global Event Structure and Tuning of Model Parameters}

In this section event-shape and charged particle inclusive distributions are presented. The analysis here represents an update of Ref. [26]. The distributions are used along with other measurements of identified hadrons to tune the parameters of QCD event generators in Section 2.3.

\subsection{Definition of Observables}

Distributions of the following event-shape variables,

- S, sphericity;

- A, aplanarity;

- T, thrust;

- $T_{\text {minor }}$

- $\ln \left(1 / y_{3}\right)$, where $y_{3}$ is the two-jet resolution variable (see below);

- $\rho=M_{h}^{2} / s$, heavy jet mass;

- C parameter;

- O, oblateness;

and the following inclusive distributions,

- $x_{p}$, scaled momentum $\left(=2|\vec{p}| / E_{c m}\right)$;

- $y$, rapidity with respect to the thrust axis;

- $p_{\perp}^{\text {in }}$, component of momentum in the event plane along $\vec{n}_{2}$ (see below);

- $p_{\perp}^{\text {out }}$, component of momentum out of the event plane along $\vec{n}_{1}$ (see below);

have been measured.

The variables above are defined in the following way. Sphericity and aplanarity are obtained from the eigenvalues of the momentum tensor $M_{\alpha \beta}=\sum_{j} p_{\alpha j} p_{\beta j}$, where $\alpha$ and $\beta$ refer to the $x$, $y$ and $z$ directions, and the sum is carried out over all of the selected particles in the event. Normalizing the eigenvalues $Q_{i}$ such that $Q_{1}+Q_{2}+Q_{3}=1$, and ordering them such that $0<Q_{1}<Q_{2}<Q_{3}$, one defines the sphericity as $S=\frac{3}{2}\left(Q_{1}+Q_{2}\right)$ and the aplanarity as $A=\frac{3}{2} Q_{1}$. The eigenvector $\vec{n}_{3}$ determines the sphericity axis, and $\vec{n}_{2}$ and $\vec{n}_{3}$ define the event plane. The sphericity $(0 \leq S \leq 1)$ approaches zero for extreme two-jet events and unity for spherical events. The aplanarity $(0 \leq A \leq 0.5)$ is a measure of event flatness, approaching zero for planar events.

The thrust of an event is defined as $T=\max \left(\sum_{j}\left|p_{|| j}\right| / \sum_{j}\left|p_{j}\right|\right)$ where the sum is over all the selected particles in the event and $p_{\|}$refers to the momentum component along the axis for 
which $T$ is maximum (the thrust axis). The direction perpendicular to the thrust axis relative to which the corresponding sum of parallel momenta is maximized is called the major axis, and the axis perpendicular to the thrust and major axes is the minor axis. The major and minor values are defined as $T_{\text {major(minor) }}=\sum_{j}\left|p_{\|, j}\right| / \sum_{j}\left|p_{j}\right|$ where $p_{\|}$is the momentum component along the major (minor) axis. The thrust $(0.5<T<1)$ approaches unity for extreme two-jet events, and the minor value approaches zero for planar events. The oblateness $O$ is defined as $O=T_{\text {major }}-T_{\text {minor }}$.

The heavy jet mass is defined by first separating the event into two hemispheres by means of a plane perpendicular to the thrust axis and computing the invariant mass of the particles in each. The larger of the two masses is $M_{h}$, and the event-shape variable $\rho$ is defined as $M_{h}^{2} / s$.

The $C$ parameter is defined as $C=3\left(\lambda_{1} \lambda_{2}+\lambda_{1} \lambda_{3}+\lambda_{2} \lambda_{3}\right)$, where $\lambda_{1}, \lambda_{2}$, and $\lambda_{3}$ are the eigenvalues of the linear momentum tensor $M_{\alpha \beta}^{\prime}=\sum_{i} \frac{p_{i \alpha} p_{i \beta}}{\left|p_{i}\right|} / \sum_{i}\left|p_{i}\right|$.

The single particle inclusive distributions of transverse momentum in and out of the event plane provide an additional measure of the overall event shape. These can be defined as $p_{\perp}^{i n}=\left|\vec{p} \cdot \vec{n}_{2}\right|$ and $p_{\perp}^{\text {out }}=\left|\vec{p} \cdot \vec{n}_{1}\right|$ where $\vec{n}_{1}$ and $\vec{n}_{2}$ are normalized eigenvectors of the momentum tensor.

The rapidity of a particle is defined as $y=\frac{1}{2} \ln \left[\left(E+p_{\|}\right) /\left(E-p_{\|}\right)\right]$. Here, $p_{\|}$refers to the component of the momentum parallel to the thrust axis, and the energy, $E$, is obtained from the particle's momentum assuming the pion mass. The detector corrections are then constructed such that the final corrected distribution corresponds to the true hadron masses.

The event-shape variable $y_{3}$ as well as the $n$-jet rates for $n=2,3,4,5$ are obtained using a jet clustering algorithm. Such algorithms are used in a number of the analyses in this paper and therefore will be defined in some detail here. For each pair of particles $i$ and $j$ in an event one computes a measure of their "distance" $y_{i j}$. Two distance measures or metrics are commonly used. In the so-called JADE algorithm [27] it is defined as

$$
y_{i j}=\frac{2 E_{i} E_{j}\left(1-\cos \theta_{i j}\right)}{E_{v i s}^{2}},
$$

where $E_{i}$ and $E_{j}$ are the particles' energies, $\theta_{i j}$ their opening angle, and $E_{v i s}$ the total energy of all of the particles used in the event. In the so-called Durham (or " $k_{\perp}$ ") algorithm [28] the distance measure is defined as

$$
y_{i j}=\frac{2 \min \left(E_{i}^{2}, E_{j}^{2}\right)\left(1-\cos \theta_{i j}\right)}{E_{v i s}^{2}} .
$$

The pair of particles with the smallest value of $y_{i j}$ is replaced by a pseudoparticle (cluster). The four-momentum of the cluster is then computed according to one of several recombination schemes. Most commonly used is the "E" scheme, where the four momentum of the cluster is taken to be the sum of the four momenta of particles $i$ and $j, p^{\mu}=p_{i}^{\mu}+p_{j}^{\mu}$. Another possibility is the "E0" scheme, where the energy of the cluster is given by the sum $E=E_{i}+E_{j}$, and the cluster momentum is given by scaling the three components of $\vec{p}_{i}+\vec{p}_{j}$ such that the invariant mass of the cluster is zero. Similarly, in the "P0" scheme the momenta are added and the energy sum $E_{i}+E_{j}$ is scaled to give a massless cluster.

The clustering procedure is repeated until all of the $y_{i j}$ are greater than a given threshold, $y_{c u t}$ (the jet resolution parameter). The number of jets is defined to be the number of remaining clusters. Alternatively one can use the algorithm to define the event-shape variable $y_{3}$ by 
continuing the clustering until exactly three clusters remain. The smallest value of $y_{i j}$ in this configuration is defined as $y_{3}$. In this way one obtains a single number for each event, whose distribution is sensitive to the probability of hard gluon radiation leading to a three-jet topology.

\subsection{Analysis Technique and Results}

The analysis is based on charged particle tracks (except for the $n$-jet rates; see below), since this allows for an accurate tuning of model parameters. The track and event selection followed the description in Section 1.3.1. In addition, for events with 5 or 6 tracks it was required that the invariant mass of at least one hemisphere be greater than the $\tau$ mass. This reduces the background from $\tau^{+} \tau^{-}$events to negligible levels, and results in 571800 accepted events from the 1992 data taking period at a centre of mass energy $E_{c m}=91.2 \mathrm{GeV}$.

In order to be able to compare more easily with other experiments, the $n$-jet rates were computed using both charged and neutral particles (see Section 1.2.6). Hadronic events were selected by requiring a total visible energy of at least $50 \%$ of $E_{c m}$, at least 15 reconstructed particles (charged or neutral), and that the polar angle of the thrust axis be in the range $30^{\circ}<\theta_{\text {thrust }}<150^{\circ}$. The cut on the number of particles effectively eliminates background from $\tau^{+} \tau^{-}$events.

For the single particle inclusive distributions, corrections for detector related effects were made by means of bin-by-bin factors as described in Section 1.3.2. For these distributions the bin size was chosen to be always at least twice as large as the detector resolution. For the charged particle distributions of $y, p_{\perp}^{\text {in }}$ and $p_{\perp}^{\text {out }}$, the correction factors were constructed such that the event axis and event plane correspond to charged particles only. Multiplicative correction factors were also applied to the $n$-jet rates.

The event-shape distributions were corrected by means of a matrix method. As with the technique of bin-by-bin factors, the matrix method used here introduces a certain model dependence; this is taken into account when estimating the systematic errors. In order to minimize this model dependence, the distributions were not corrected to account for neutral particles, i.e. the results represent what would be obtained with charged particles only. The matrix method allows one to use smaller bin sizes than would be reliable with bin-by-bin corrections; they were chosen here to be somewhat smaller than twice the corresponding detector resolution.

The matrix correction method was performed in the following way. Monte Carlo events passing the event selection criteria were used to fill a two-dimensional histogram. The probability that an event is generated with $x_{\text {gen }}$ in interval $j$, given that it is observed with $x_{r e c}$ in interval $i$, is given by

$$
B_{j i}=\frac{H_{i j}}{\sum_{k} H_{i k}},
$$

where $H_{i j}$ is the number of events where the pair $\left(x_{r e c}, x_{g e n}\right)$ falls into the interval $(i, j)$. The matrix $B$ relates the generated and reconstructed Monte Carlo distributions,

$$
D_{j, g e n}^{M C}(x) \Delta x_{j}=\sum_{i} B_{j i} D_{i, r e c}^{M C}(x) \Delta x_{i},
$$

where $\Delta x$ is the bin width. This can be used to correct the data,

$$
D_{j, \text { corr }}^{\text {data }}(x) \Delta x_{j}=\bar{C}_{i}(x) \sum_{i} B_{j i} D_{i, r e c}^{\text {data }}(x) \Delta x_{i},
$$


where the additional factor

$$
\bar{C}_{i}(x)=\frac{D_{i, g e n}^{M C}(\text { no cuts, no ISR })}{D_{i, g e n}^{M C}(c u t s, I S R)}
$$

corrects for initial state radiation (ISR) as well as for the fact that the generated distribution depends on the event selection cuts. This procedure is to first approximation independent of the Monte Carlo generator used. A possible model dependence cannot be excluded, however, since the matrix $B$ depends on the input Monte Carlo distribution. It has been found that the differences between the results of the matrix method and those of the bin-by-bin factors method are of the order of the errors of the data.

Systematic uncertainties have been estimated by individually varying all track and event selection cuts. The maximum change in each bin with respect to the standard set of cuts is included in the systematic error. The largest sources of error (approximately $0.5 \%$ ) were found to be in the low momentum region $\left(x_{p} \approx 0.01\right)$ when changing the cuts on the number of TPC coordinates (from 4 to 7 ) and on the minimum transverse momentum (from $0.2 \mathrm{GeV}$ to 0.3 $\mathrm{GeV}$ ). In addition, a systematic error due to model dependence was estimated by computing simplified correction factors based on the models JETSET [25, 29], ARIADNE [30] and HERWIG, [31] as described in Section 1.3.2. The difference between the JETSET and HERWIG correction factors gave approximately a $1 \%$ error in the $x_{p}$ distribution around $x_{p} \approx 0.01$, and a $1-2 \%$ error in the event-shape distributions in the heavily populated regions. The total systematic error is given as the quadratic sum of the contributions from cut dependence and model dependence, and the bin-to-bin fluctuations of the errors have been smoothed.

As a further check, the ratio of positive to negative particle rates was found to be reproduced by the detector simulation to better than $0.6 \%$ overall and to within $1.2 \%$ at low momenta. The reported distributions give the summed contributions of positive and negative particles, and the uncertainty resulting from this check is small compared to the other errors given above.

The results are shown in Figs. 5 through 16 together with the predictions of several Monte Carlo models. The error bars are the quadratic sum of statistical and systematic uncertainties. Parameters of the models have been tuned by means of a comparison with the data presented in this section as well as with distributions of identified hadrons. The models and the parameter tuning procedure are described in Section 2.3. The measured values are given in Tables 1 through 6.

From the figures one can see that the data and Monte Carlo predictions are qualitatively in good agreement. The question of model comparisons is taken up in greater detail in the next section, after the models and the tuning of their parameters have been described.

\subsection{Tuning of QCD Models}

Most of the analyses presented in this paper involve in one way or another comparisons with the predictions of Monte Carlo models. Such models involve a number of parameters whose values must be determined by comparisons with data. In this section several Monte Carlo models are considered and the optimization of their parameters is described. 

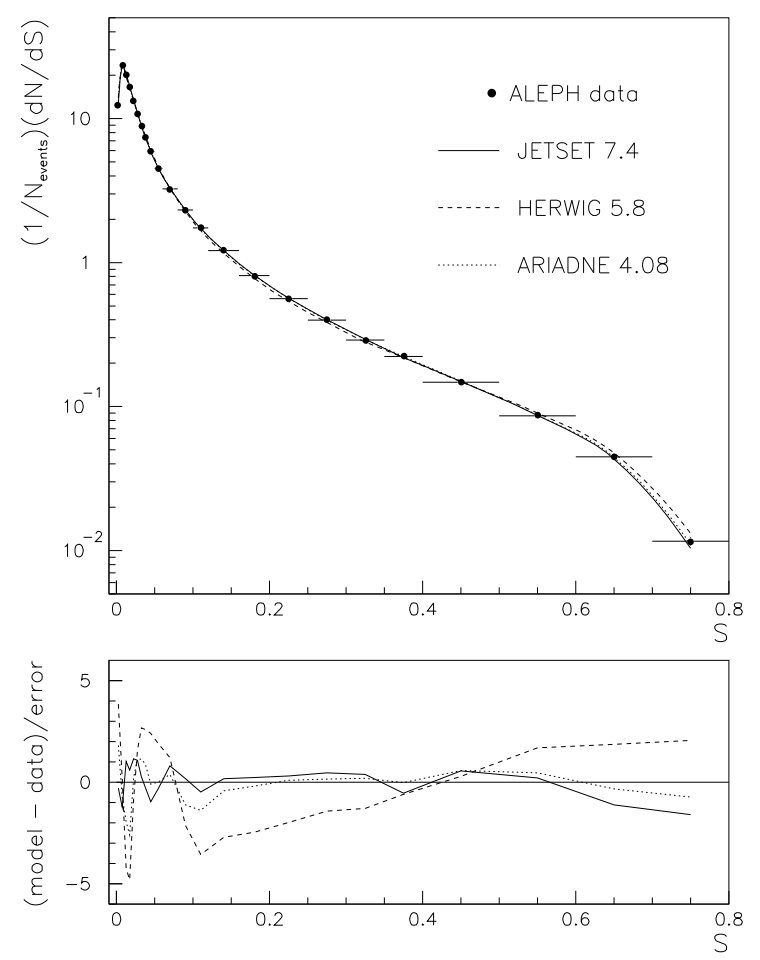

Figure 5: The sphericity distribution.
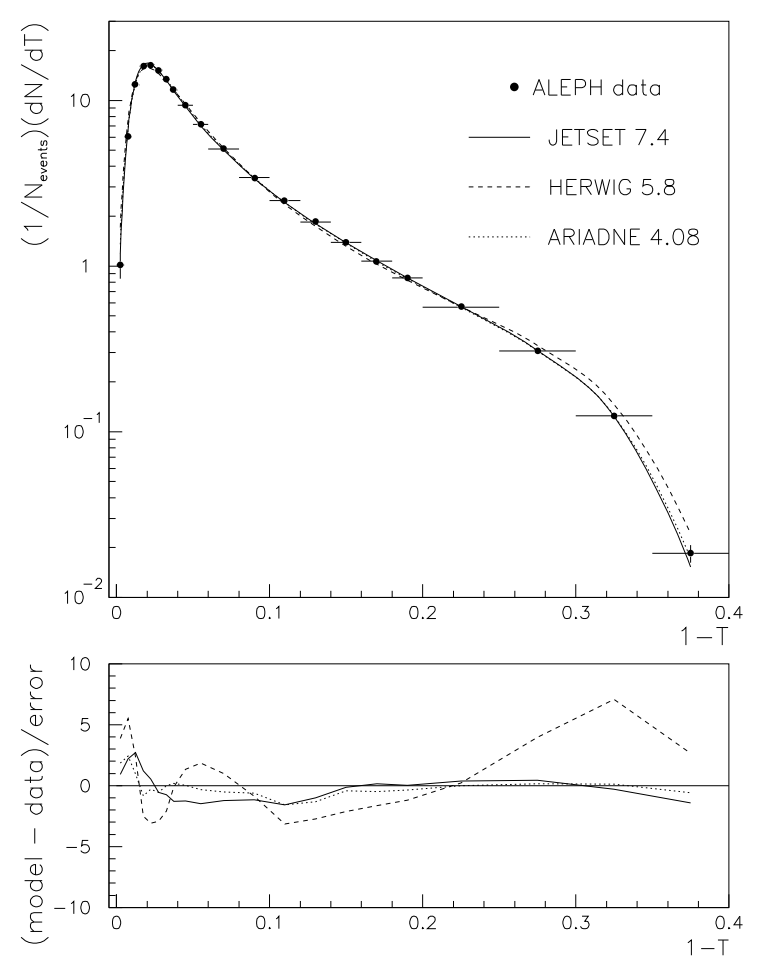

Figure 7: The thrust distribution.
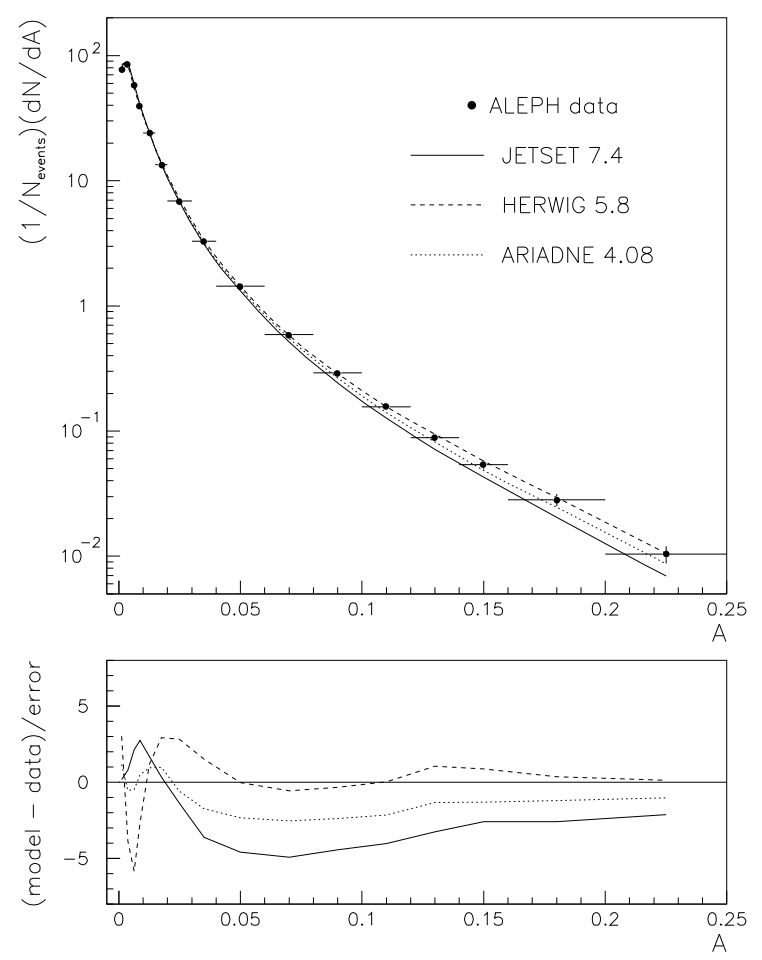

Figure 6: The aplanarity distribution.
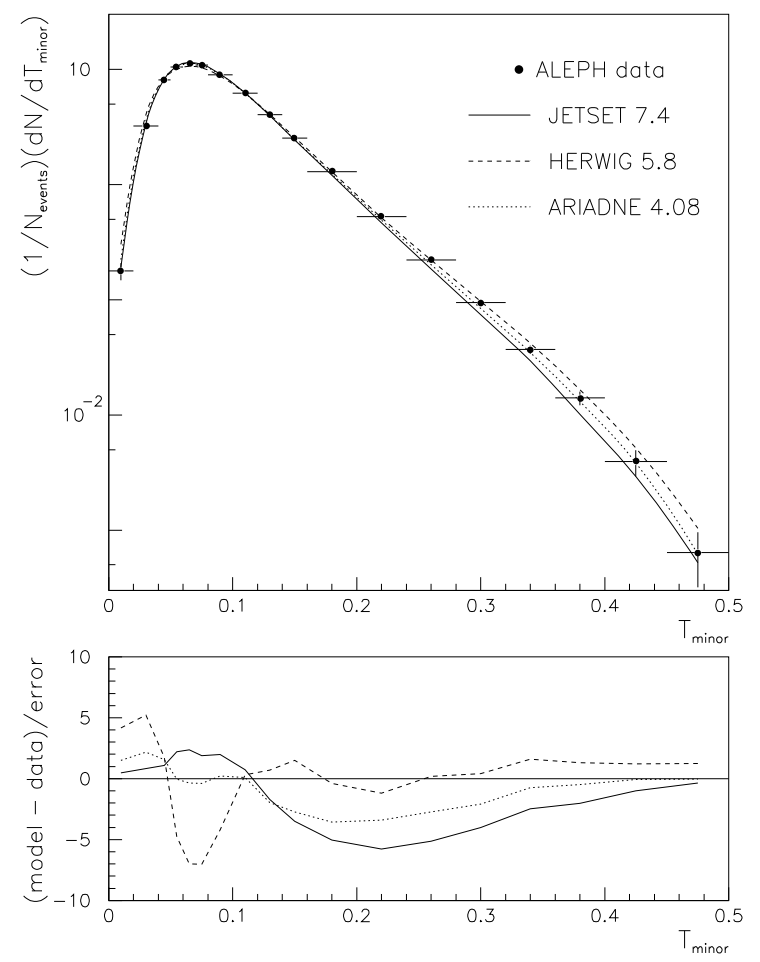

Figure 8: The minor distribution. 

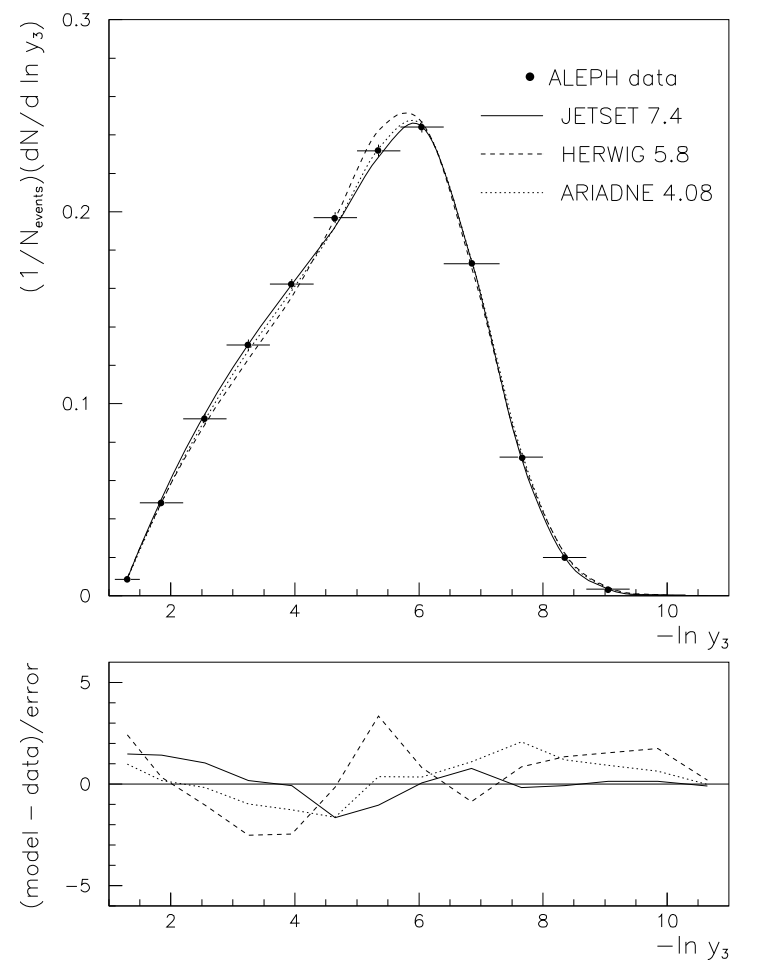

Figure 9: The distribution of $-\ln y_{3}$.
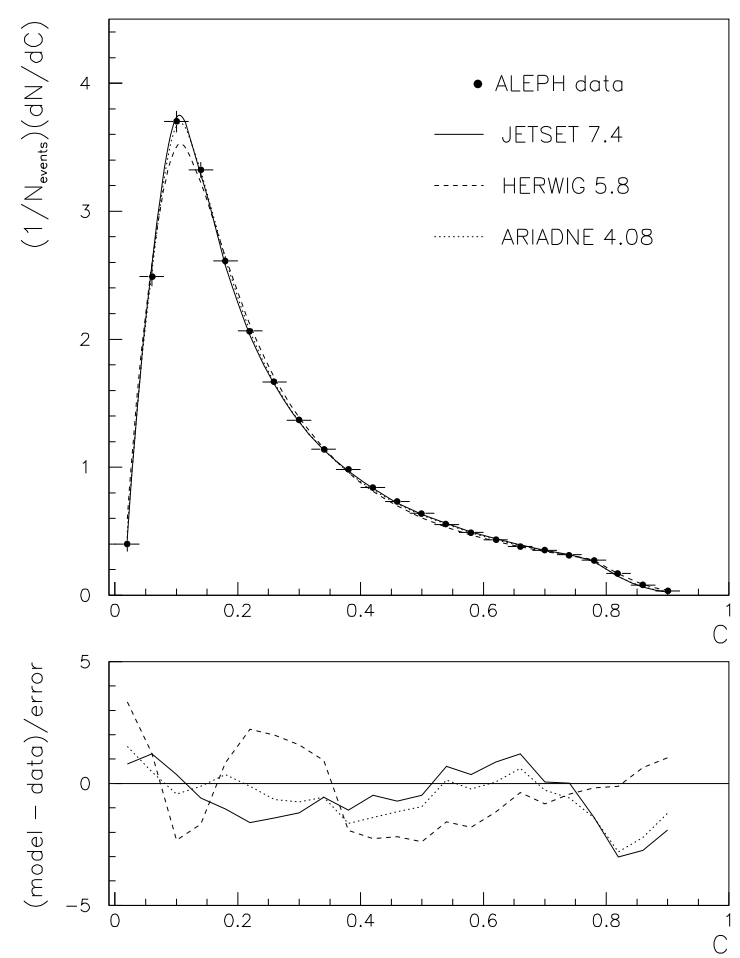

Figure 11: The distribution of $C$ parameter.
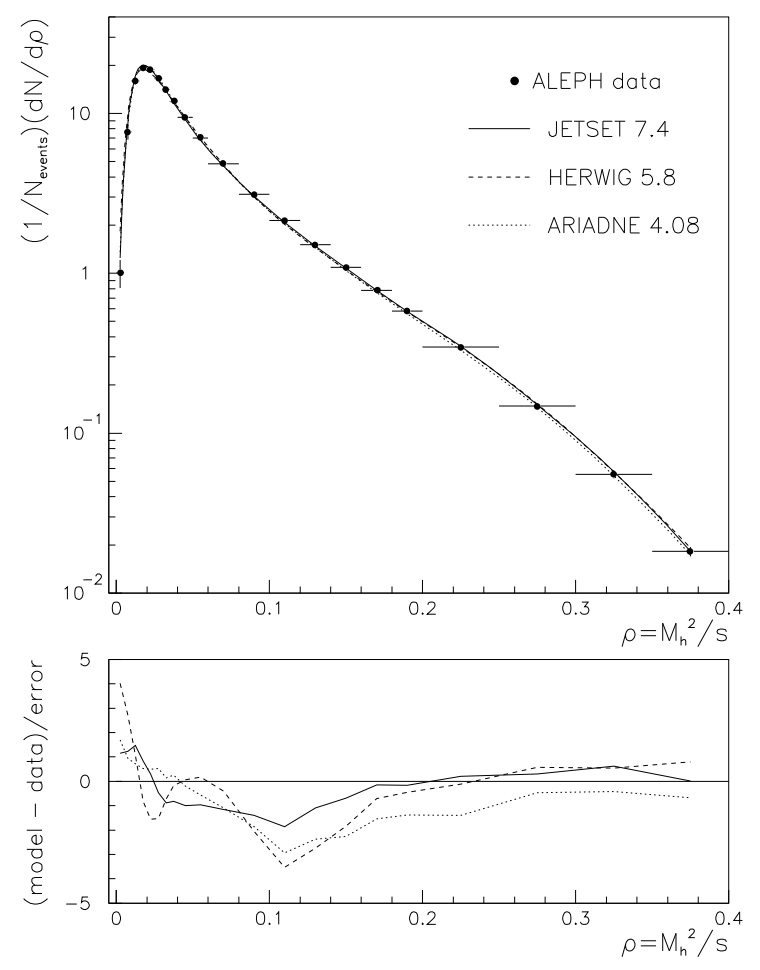

Figure 10: The distribution of $\rho=M_{h}^{2} / s$.
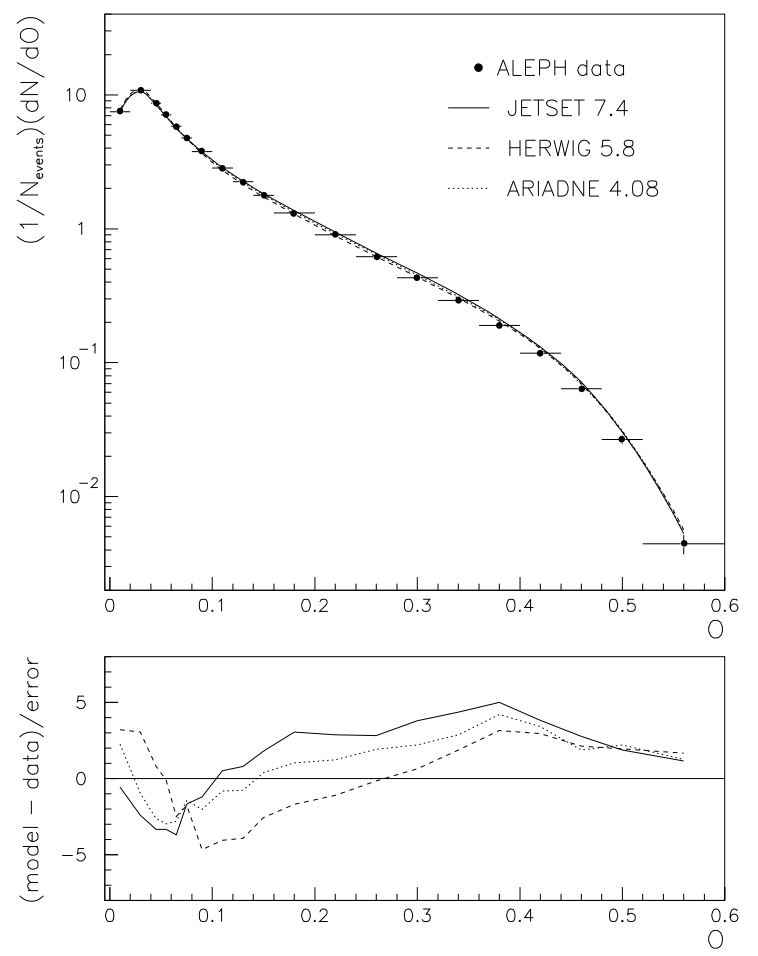

Figure 12: The distribution of oblateness. 

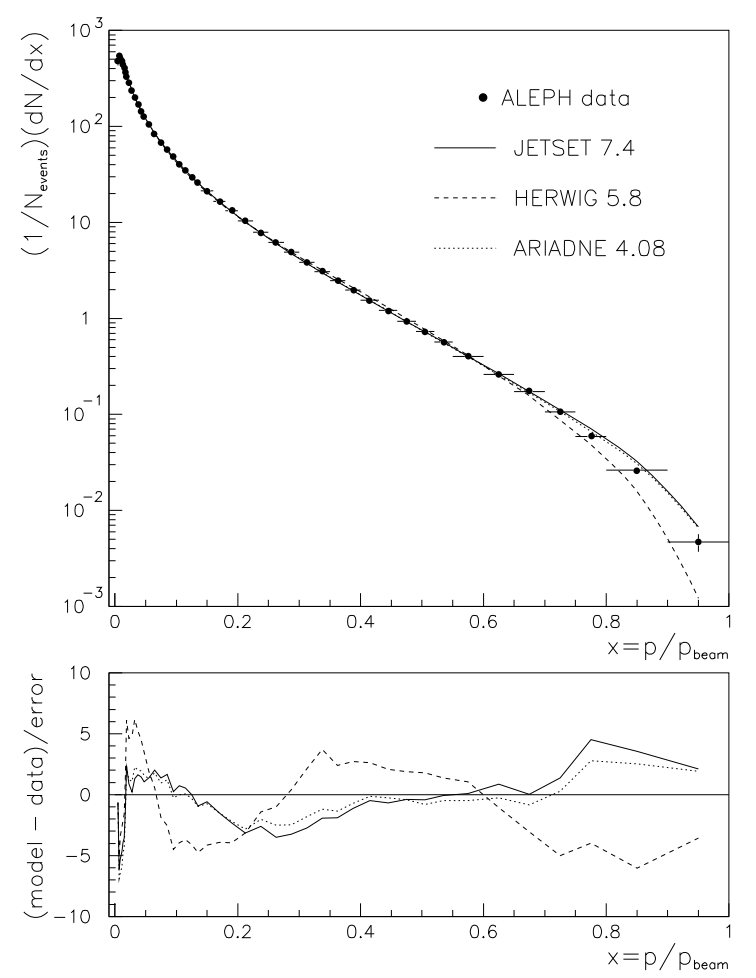

Figure 13: The inclusive distribution of $x_{p}=$ $p / p_{\text {beam }}$ for charged particles.
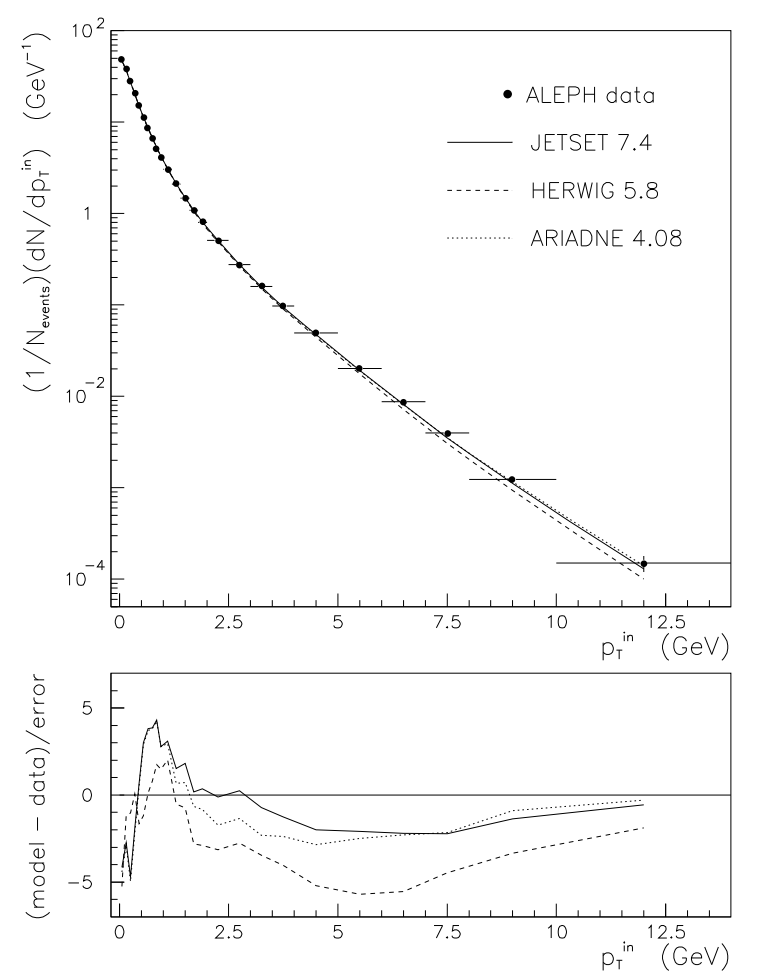

Figure 15: The inclusive distribution of $p_{\perp}^{\text {in }}$ for charged particles, using the event plane based on the sphericity tensor.
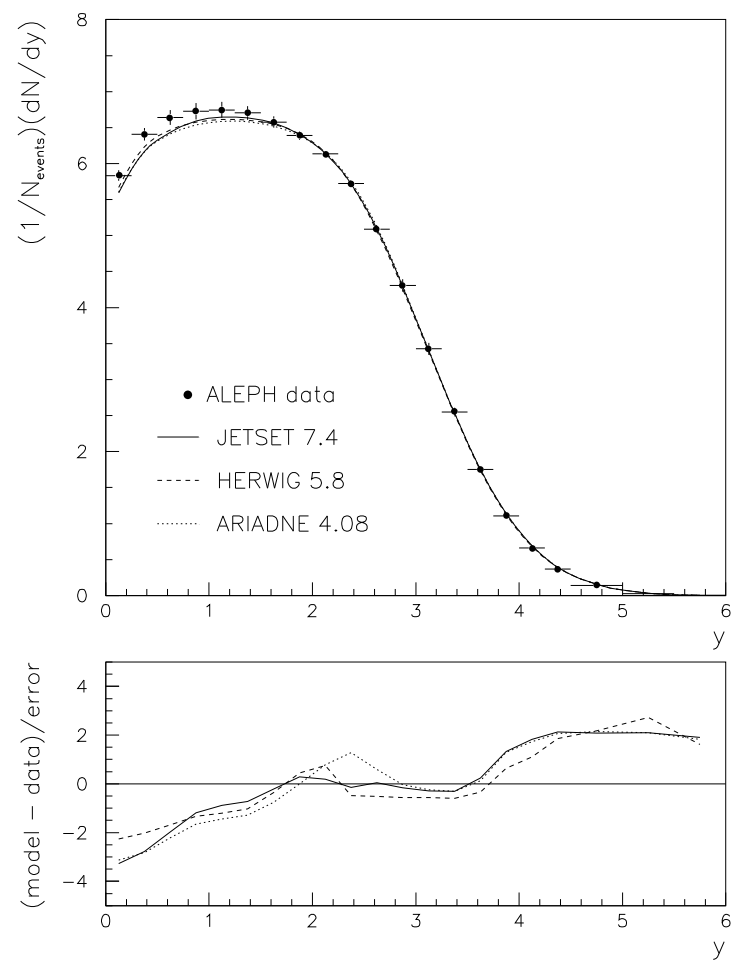

Figure 14: The inclusive distribution of rapidity $y$ for charged particles with respect to the thrust axis.
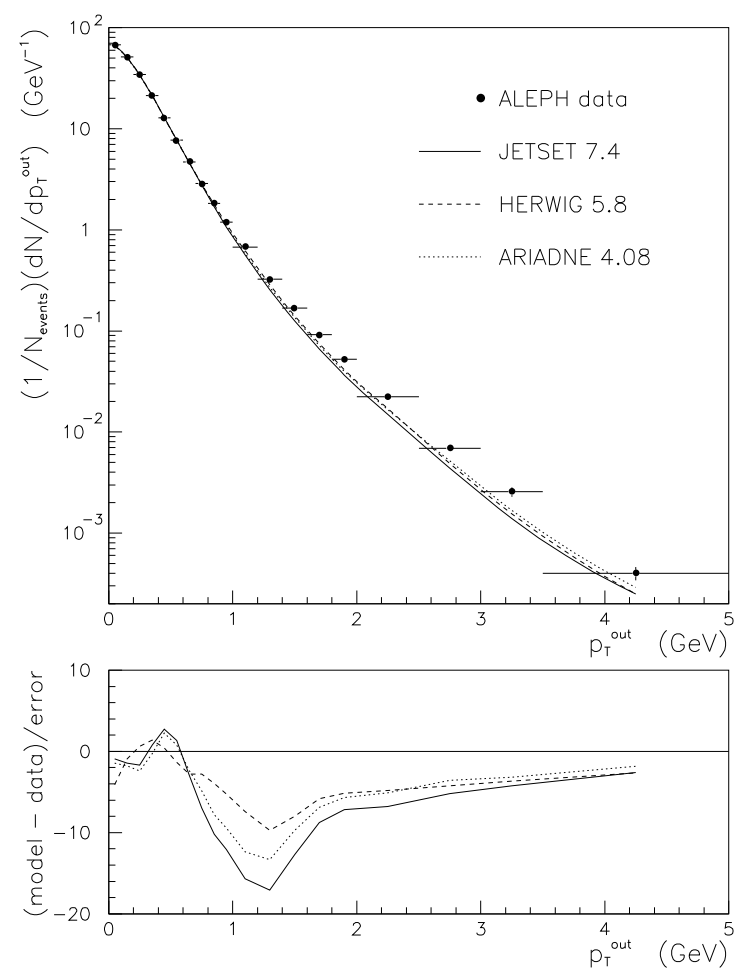

Figure 16: The inclusive distribution of $p_{\perp}^{\text {out }}$ for charged particles, using the event plane based on the sphericity tensor. 


\begin{tabular}{|c|c|c|c|}
\hline Interval & $(1 / N)(d N / d S) \pm$ stat. \pm sys. & & \\
\hline $0.000-0.005$ & $12.36 \pm 0.08 \pm 0.40$ & & \\
\hline $0.005-0.010$ & $23.33 \pm 0.11 \pm 0.23$ & & \\
\hline $0.010-0.015$ & $20.23 \pm 0.10 \pm 0.12$ & Interval & $(1 / N)(d N / d A)+$ stat + \\
\hline $0.015-0.020$ & $16.69 \pm 0.09 \pm 0.08$ & $00000-00025$ & 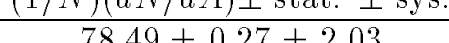 \\
\hline $0.020-0.025$ & $13.41 \pm 0.08 \pm 0.06$ & $\begin{array}{l}0.0000-0.0020 \\
0.0025-0.9050\end{array}$ & $18.49 \pm 0.26 \pm 2.05$ \\
\hline $0.025-0.030$ & $10.79 \pm 0.07 \pm 0.07$ & $0.0025-0.0050$ & $85.98 \pm 0.28 \pm 1.25$ \\
\hline $0.030-0.035$ & $8.870 \pm 0.066 \pm 0.067$ & $0.0050-0.0075$ & $58.23 \pm 0.23 \pm 0.47$ \\
\hline $0.035-0.040$ & $7.408 \pm 0.060 \pm 0.066$ & $0.0075-0.0100$ & $39.49 \pm 0.19 \pm 0.26$ \\
\hline $0.040-0.050$ & $5.922 \pm 0.038 \pm 0.058$ & 100 & $24.02 \pm 0.11 \pm 0.21$ \\
\hline $0.050-0.060$ & $4.508 \pm 0.033 \pm 0.041$ & 0.0150 & $13.46 \pm 0$ \\
\hline $0.060-0.080$ & $3.258 \pm 0.020 \pm 0.023$ & .0500 & \pm 0.088 \\
\hline $0.080-0.100$ & $2.317 \pm 0.017 \pm 0.016$ & $\begin{array}{r}0.0300-0.0400 \\
0.0400-0.9600\end{array}$ & $\begin{array}{l}3.285 \pm 0.028 \pm 0.042 \\
1428+0.21+0.090\end{array}$ \\
\hline $0.100-0.120$ & $1.742 \pm 0.015 \pm 0.017$ & $\begin{array}{l}0.0400-0.0000 \\
0.0600-0.0800\end{array}$ & $\begin{array}{c}1.430 \pm 0.013 \pm 0.022 \\
05900+00086+00130\end{array}$ \\
\hline $0.120-0.160$ & $1.211 \pm 0.009 \pm 0.016$ & $0.0800-0.7000$ & ..5900 工 U.0000 工 U.01.00 \\
\hline $0.160-0.200$ & $0.8132 \pm 0.0070 \pm 0.0135$ & $\begin{array}{r}0.0800-0.1000 \\
0.1000-0.1900\end{array}$ & $0.2908 \pm 0.0062 \pm 0.0089$ \\
\hline $0.200-0.250$ & $0.5626 \pm 0.0052 \pm 0.0116$ & $0.1200-0.1400$ & 0.1004 工 0.0040 工 0.0000 \\
\hline $0.250-0.300$ & $0.3973 \pm 0.0043 \pm 0.0093$ & $01400-0.1600$ & 0.0000 工 0.0000 工 0.0041 \\
\hline $0.300-0.350$ & $0.2903 \pm 0.0036 \pm 0.0069$ & $\begin{array}{r}-0.1000 \\
-0.2000\end{array}$ & $\begin{array}{l} \pm 0.0026 \pm 0.0034 \\
+0.0014+0.0027\end{array}$ \\
\hline $0.350-0.400$ & $0.2224 \pm 0.0032 \pm 0.0053$ & $\begin{array}{c}0.1000-0.2000 \\
0.9000-0.9500\end{array}$ & $\begin{array}{l}0.0200 \\
0.0104+0.0014 \\
0\end{array}$ \\
\hline $0.400-0.500$ & $0.1476 \pm 0.0019 \pm 0.0035$ & 0.2500 & .0014 \\
\hline $0.500-0.600$ & $0.0861 \pm 0.0014 \pm 0.0020$ & & \\
\hline $0.600-0.700$ & $0.0447 \pm 0.0010 \pm 0.0011$ & & \\
\hline $0.700-0.800$ & $0.0116 \pm 0.0005 \pm 0.0006$ & & \\
\hline
\end{tabular}

Table 1: Distributions of sphericity $S$ and aplanarity $A$.

\begin{tabular}{|c|c|c|c|}
\hline Interval & $(1 / N)(d N / d T) \pm$ stat. \pm sys. & & \\
\hline $0.000-0.005$ & $1.017 \pm 0.022 \pm 0.178$ & Interval & $(1 / N)\left(d N / d T_{\text {minor }}\right)+$ stat. + svs. \\
\hline $0.005-0.010$ & $6.035 \pm 0.054 \pm 0.272$ & & 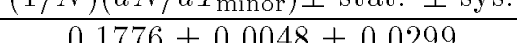 \\
\hline $0.010-0.015$ & $12.44 \pm 0.08 \pm 0.22$ & $\begin{array}{r}0.000-0.020 \\
0.020-0040\end{array}$ & $0.16 r 6 \pm 0.0048 \pm 0.0299$ \\
\hline $0.015-0.020$ & $16.07 \pm 0.09 \pm 0.22$ & $\begin{array}{l}0.020-0.040 \\
0.040-0.050\end{array}$ & $3.236 \pm 0.020 \pm 0.101$ \\
\hline $0.020-0.025$ & $16.45 \pm 0.09 \pm 0.26$ & $\begin{aligned} 0.050 \\
0.060\end{aligned}$ & $8.106 \pm 0.044 \pm 0.128$ \\
\hline $0.025-0.030$ & $15.25 \pm 0.08 \pm 0.22$ & $0.000-0.000$ & $10.45 \pm 0.05 \pm 0.07$ \\
\hline $0.030-0.035$ & $13.38 \pm 0.08 \pm 0.16$ & $0.060-$ & $11.27 \pm 0.05 \pm 0.08$ \\
\hline $0.035-0.040$ & $11.58 \pm 0.07 \pm 0.12$ & .080 & $10.89 \pm 0.05 \pm 0.07$ \\
\hline $0.040-0.050$ & $9.346 \pm 0.047 \pm 0.100$ & 0.080 & $\begin{array}{l}9.003 \pm 0.031 \pm 0.052 \\
6208+0.026+0.040\end{array}$ \\
\hline $0.050-0.060$ & $7.159 \pm 0.041 \pm 0.076$ & $0.100-0.120$ & $\begin{array}{l}0.208 \pm 0.020 \pm 0.040 \\
4043+0021+0030\end{array}$ \\
\hline $0.060-0.080$ & $5.088 \pm 0.025 \pm 0.056$ & $0.120-0.140$ & $\begin{array}{l}4.040 \pm 0.021 \text { 土 } 0.0000 \\
2596+0.017+0.029\end{array}$ \\
\hline $0.080-0.100$ & $3.427 \pm 0.020 \pm 0.040$ & $\begin{array}{c}.100 \\
.200\end{array}$ & $\begin{array}{l}2.536 \pm 0.0 \\
1.200 \pm 00\end{array}$ \\
\hline $0.100-0.120$ & $2.482 \pm 0.017 \pm 0.037$ & $\begin{array}{c}0.100-0.200 \\
0.500-0.240\end{array}$ & $\begin{array}{l}1.299 \pm 0.009 \pm 0.016 \\
05960 \pm 00057 \pm 0000\end{array}$ \\
\hline $0.120-0.140$ & $1.847 \pm 0.015 \pm 0.038$ & $\begin{array}{l}0.200-0.240 \\
0.240-0.280\end{array}$ & $\begin{array}{l}0.5200 \pm 0.0036 \\
0.9208+0.0090\end{array}$ \\
\hline $0.140-0.160$ & $1.390 \pm 0.013 \pm 0.033$ & $0.280-0.390$ & $0.2208 \pm 0.0036 \pm 0.0000$ \\
\hline $0.160-0.180$ & $1.072 \pm 0.011 \pm 0.025$ & $0.200-0.020$ & 0.0940 土 0.0020 土 0.0044 \\
\hline $0.180-0.200$ & $0.8465 \pm 0.0096 \pm 0.0199$ & $\begin{array}{l}0.520-0.500 \\
0.360-0400\end{array}$ & 0.0011 土.0010 0.0026 \\
\hline $0.200-0.250$ & $0.5661 \pm 0.0051 \pm 0.0122$ & $0.400-0450$ & $0.0141 \pm 0.0009 \pm 0.0016$ \\
\hline $0.250-0.300$ & $0.3065 \pm 0.0037 \pm 0.0054$ & $150-0.400$ & $0.0069 \pm 0.0004 \pm 0.0009$ \\
\hline $0.300-0.350$ & $0.1248 \pm 0.0024 \pm 0.0019$ & .000 & \pm 0.00020 \\
\hline $0.350-0.400$ & $0.0184 \pm 0.0010 \pm 0.0020$ & & \\
\hline
\end{tabular}

Table 2: Distributions of $1-T$ and minor. 


\begin{tabular}{|c|c|}
\hline Interval & $(1 / N)\left(d N / d y_{3}\right) \pm$ stat. \pm sys. \\
\hline $1.100-1.500$ & $0.0084 \pm 0.0002 \pm 0.0003$ \\
$1.500-2.200$ & $0.0483 \pm 0.0004 \pm 0.0015$ \\
$2.200-2.900$ & $0.0921 \pm 0.0005 \pm 0.0026$ \\
$2.900-3.600$ & $0.1306 \pm 0.0006 \pm 0.0029$ \\
$3.600-4.300$ & $0.1623 \pm 0.0007 \pm 0.0026$ \\
$4.300-5.000$ & $0.1969 \pm 0.0008 \pm 0.0027$ \\
$5.000-5.700$ & $0.2318 \pm 0.0009 \pm 0.0030$ \\
$5.700-6.400$ & $0.2442 \pm 0.0009 \pm 0.0029$ \\
$6.400-7.300$ & $0.1729 \pm 0.0007 \pm 0.0017$ \\
$7.300-8.000$ & $0.0721 \pm 0.0005 \pm 0.0014$ \\
$8.000-8.700$ & $0.0200 \pm 0.0003 \pm 0.0015$ \\
$8.700-9.400$ & $0.0034 \pm 0.0001 \pm 0.0006$ \\
$9.400-10.300$ & $0.00036 \pm 0.00003 \pm 0.00012$ \\
$10.300-11.000$ & $0.00004 \pm 0.00001 \pm 0.00007$ \\
\hline
\end{tabular}

\begin{tabular}{|c|c|}
\hline Interval & $(1 / N)(d N / d \rho) \pm$ stat. \pm sys. \\
\hline $0.000-0.005$ & $1.011 \pm 0.022 \pm 0.202$ \\
$0.005-0.010$ & $7.656 \pm 0.060 \pm 0.818$ \\
$0.010-0.015$ & $15.90 \pm 0.08 \pm 0.83$ \\
$0.015-0.020$ & $19.36 \pm 0.09 \pm 0.71$ \\
$0.020-0.025$ & $18.73 \pm 0.09 \pm 0.57$ \\
$0.025-0.030$ & $16.52 \pm 0.09 \pm 0.40$ \\
$0.030-0.035$ & $14.07 \pm 0.08 \pm 0.32$ \\
$0.035-0.040$ & $11.89 \pm 0.07 \pm 0.32$ \\
$0.040-0.050$ & $9.422 \pm 0.047 \pm 0.312$ \\
$0.050-0.060$ & $7.013 \pm 0.040 \pm 0.243$ \\
$0.060-0.080$ & $4.839 \pm 0.024 \pm 0.140$ \\
$0.080-0.100$ & $3.125 \pm 0.019 \pm 0.062$ \\
$0.100-0.120$ & $2.138 \pm 0.015 \pm 0.029$ \\
$0.120-0.140$ & $1.506 \pm 0.013 \pm 0.020$ \\
$0.140-0.160$ & $1.089 \pm 0.011 \pm 0.019$ \\
$0.160-0.180$ & $0.7835 \pm 0.0091 \pm 0.0159$ \\
$0.180-0.200$ & $0.5794 \pm 0.0078 \pm 0.0133$ \\
$0.200-0.250$ & $0.3466 \pm 0.0039 \pm 0.0108$ \\
$0.250-0.300$ & $0.1482 \pm 0.0025 \pm 0.0067$ \\
$0.300-0.350$ & $0.0554 \pm 0.0015 \pm 0.0029$ \\
$0.350-0.400$ & $0.0182 \pm 0.0008 \pm 0.0009$ \\
\hline
\end{tabular}

Table 3: Distributions of $-\ln y_{3}$ and $\rho=M_{h}^{2} / s$.

\begin{tabular}{|c|c|}
\hline Interval & $(1 / N)(d N / d C) \pm$ stat. \pm sys. \\
\hline $0.000-0.040$ & $0.4008 \pm 0.0046 \pm 0.0581$ \\
$0.040-0.080$ & $2.490 \pm 0.011 \pm 0.075$ \\
$0.080-0.120$ & $3.701 \pm 0.014 \pm 0.083$ \\
$0.120-0.160$ & $3.323 \pm 0.014 \pm 0.060$ \\
$0.160-0.200$ & $2.613 \pm 0.012 \pm 0.034$ \\
$0.200-0.240$ & $2.065 \pm 0.011 \pm 0.019$ \\
$0.240-0.280$ & $1.666 \pm 0.010 \pm 0.012$ \\
$0.280-0.320$ & $1.368 \pm 0.009 \pm 0.008$ \\
$0.320-0.360$ & $1.142 \pm 0.008 \pm 0.007$ \\
$0.360-0.400$ & $0.9815 \pm 0.0077 \pm 0.0090$ \\
$0.400-0.440$ & $0.8419 \pm 0.0071 \pm 0.0120$ \\
$0.440-0.480$ & $0.7324 \pm 0.0066 \pm 0.0144$ \\
$0.480-0.520$ & $0.6394 \pm 0.0061 \pm 0.0140$ \\
$0.520-0.560$ & $0.5518 \pm 0.0056 \pm 0.0118$ \\
$0.560-0.600$ & $0.4897 \pm 0.0053 \pm 0.0102$ \\
$0.600-0.640$ & $0.4322 \pm 0.0049 \pm 0.0093$ \\
$0.640-0.680$ & $0.3817 \pm 0.0046 \pm 0.0087$ \\
$0.680-0.720$ & $0.3499 \pm 0.0045 \pm 0.0082$ \\
$0.720-0.760$ & $0.3156 \pm 0.0046 \pm 0.0074$ \\
$0.760-0.800$ & $0.2742 \pm 0.0056 \pm 0.0073$ \\
$0.800-0.840$ & $0.1691 \pm 0.0056 \pm 0.0057$ \\
$0.840-0.880$ & $0.0804 \pm 0.0048 \pm 0.0038$ \\
$0.880-0.920$ & $0.0339 \pm 0.0049 \pm 0.0025$ \\
$0.920-1.000$ & $0.0059 \pm 0.0025 \pm 0.0007$ \\
\hline
\end{tabular}

\begin{tabular}{|c|c|}
\hline Interval & $(1 / N)(d N / d O) \pm$ stat. \pm sys. \\
\hline $0.000-0.020$ & $7.480 \pm 0.030 \pm 0.047$ \\
$0.020-0.040$ & $10.80 \pm 0.03 \pm 0.07$ \\
$0.040-0.050$ & $8.679 \pm 0.045 \pm 0.061$ \\
$0.050-0.060$ & $7.107 \pm 0.041 \pm 0.051$ \\
$0.060-0.070$ & $5.816 \pm 0.037 \pm 0.038$ \\
$0.070-0.080$ & $4.776 \pm 0.033 \pm 0.027$ \\
$0.080-0.100$ & $3.775 \pm 0.021 \pm 0.019$ \\
$0.100-0.120$ & $2.841 \pm 0.018 \pm 0.018$ \\
$0.120-0.140$ & $2.243 \pm 0.016 \pm 0.019$ \\
$0.140-0.160$ & $1.783 \pm 0.014 \pm 0.018$ \\
$0.160-0.200$ & $1.315 \pm 0.009 \pm 0.016$ \\
$0.200-0.240$ & $0.9010 \pm 0.0071 \pm 0.0141$ \\
$0.240-0.280$ & $0.6210 \pm 0.0058 \pm 0.0119$ \\
$0.280-0.320$ & $0.4302 \pm 0.0048 \pm 0.0087$ \\
$0.320-0.360$ & $0.2917 \pm 0.0039 \pm 0.0057$ \\
$0.360-0.400$ & $0.1892 \pm 0.0031 \pm 0.0037$ \\
$0.400-0.440$ & $0.1175 \pm 0.0025 \pm 0.0025$ \\
$0.440-0.480$ & $0.0639 \pm 0.0018 \pm 0.0020$ \\
$0.480-0.520$ & $0.0267 \pm 0.0011 \pm 0.0016$ \\
$0.520-0.600$ & $0.0044 \pm 0.0003 \pm 0.0007$ \\
\hline
\end{tabular}

Table 4: Distributions of $C$ parameter and oblateness $O$. 


\begin{tabular}{|c|c|c|c|}
\hline Interval & $(1 / N)\left(d N / d x_{p}\right) \pm$ stat. \pm sys. & & \\
\hline $0.004-0.006$ & $478.6 \pm 1.1 \pm 48.0$ & & \\
\hline $0.006-0.008$ & $535.8 \pm 1.0 \pm 6.8$ & & \\
\hline $0.008-0.010$ & $513.0 \pm 1.0 \pm 4.5$ & & \\
\hline $0.010-0.012$ & $478.6 \pm 0.9 \pm 3.3$ & & \\
\hline $0.012-0.014$ & $440.2 \pm 0.9 \pm 2.7$ & & \\
\hline $0.014-0.016$ & $403.1 \pm 0.8 \pm 2.1$ & & \\
\hline $0.016-0.018$ & $364.4 \pm 0.8 \pm 1.5$ & & \\
\hline $0.018-0.020$ & $329.5 \pm 0.7 \pm 1.1$ & & \\
\hline $0.020-0.025$ & $287.2 \pm 0.5 \pm 1.2$ & & \\
\hline $0.025-0.030$ & $237.3 \pm 0.4 \pm 1.0$ & & \\
\hline $0.030-0.035$ & $198.1 \pm 0.4 \pm 0.8$ & & \\
\hline $0.035-0.040$ & $168.7 \pm 0.3 \pm 0.8$ & Interval & $(1 / N)(d N(d u)+$ \\
\hline $0.040-0.045$ & $145.4 \pm 0.3 \pm 0.7$ & $0000-0950$ & 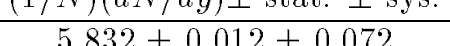 \\
\hline $0.045-0.050$ & $127.0 \pm 0.3 \pm 0.6$ & $\begin{array}{r}0.000-0.200 \\
0.250-0.500\end{array}$ & $\begin{array}{l}0.832 \pm 0.012 \pm 0.012 \\
6407+0013+0086\end{array}$ \\
\hline $0.050-0.060$ & $105.4 \pm 0.2 \pm 0.5$ & $\begin{array}{r}0.200-0.000 \\
0.500-0750\end{array}$ & 0.406 土.013 0.080 \\
\hline $0.060-0.070$ & $83.45 \pm 0.16 \pm 0.44$ & $0.750-1000$ & $6.0419+0.010 \pm 0.104$ \\
\hline $0.070-0.080$ & $68.12 \pm 0.15 \pm 0.37$ & $\begin{array}{l}0.100-1.000 \\
1000-1950\end{array}$ & $0.120 \pm 0.012 \pm 0.114$ \\
\hline $0.080-0.090$ & $56.28 \pm 0.13 \pm 0.32$ & $\begin{array}{l}1.000-1.200 \\
1.250-1500\end{array}$ & $0.140 \pm 0.012 \pm 0.110$ \\
\hline $0.090-0.100$ & $47.61 \pm 0.12 \pm 0.29$ & $\begin{array}{l}1.200-1.500 \\
1.500-1750\end{array}$ & $\begin{array}{l}0.103 \pm 0.011 \pm 0.095 \\
6.578+0.010+0078\end{array}$ \\
\hline $0.100-0.110$ & $40.31 \pm 0.11 \pm 0.28$ & $1750-2000$ & $0.018 \pm 0.010 \pm 0.018$ \\
\hline $0.110-0.120$ & $34.46 \pm 0.10 \pm 0.28$ & $\begin{array}{l}1.750-2.000 \\
2000-2.250\end{array}$ & $\begin{array}{l}0.389 \pm 0.010 \pm 0.056 \\
6.135+0000+0025\end{array}$ \\
\hline $0.120-0.130$ & $29.84 \pm 0.10 \pm 0.25$ & $\begin{array}{l}2.000-2.250 \\
2.250-2500\end{array}$ & $\begin{array}{l}0.135 \pm 0.009 \pm 0.025 \\
5721+0000+0018\end{array}$ \\
\hline $0.130-0.140$ & $26.07 \pm 0.09 \pm 0.22$ & $\begin{aligned} & 2.200-2.000 \\
& 2.500-2-750\end{aligned}$ & $0.621 \pm 0.009 \pm 0.018$ \\
\hline $0.140-0.160$ & $21.26 \pm 0.06 \pm 0.18$ & $\begin{array}{l}2.500-2.750 \\
0.750-2000\end{array}$ & $5.090 \pm 0.009 \pm 0.051$ \\
\hline $0.160-0.180$ & $16.62 \pm 0.05 \pm 0.14$ & $\begin{array}{r}2.700-3.000 \\
2000-250\end{array}$ & $\begin{array}{l}4.307 \pm 0.008 \pm 0.087 \\
2.407 \pm 0.07\end{array}$ \\
\hline $0.180-0.200$ & $13.21 \pm 0.05 \pm 0.11$ & $\begin{array}{l}3.000-3.200 \\
3.250-3.500\end{array}$ & $\begin{array}{l}3.426 \pm 0.006 \pm 0.084 \\
2549+0006+0058\end{array}$ \\
\hline $0.200-0.225$ & $10.34 \pm 0.04 \pm 0.08$ & $3500-3750$ & $1749+0.005+0036$ \\
\hline $0.225-0.250$ & $7.920 \pm 0.031 \pm 0.063$ & $\begin{array}{l}3.500-3.150 \\
3.750-4000\end{array}$ & $\begin{array}{l}1.149 \pm 0.005 \pm 0.036 \\
1.107+0004+0023\end{array}$ \\
\hline $0.250-0.275$ & $6.197 \pm 0.028 \pm 0.049$ & $\begin{array}{l}3.150-4.000 \\
4000-4.950\end{array}$ & $\begin{array}{c}1.106 \pm 0.004 \pm 0.023 \\
06623+00027+00158\end{array}$ \\
\hline $0.275-0.300$ & $4.889 \pm 0.025 \pm 0.041$ & $4.250-4.500$ & 0.0020 土 $0.00262+0.0019+0.0119$ \\
\hline $0.300-0.325$ & $3.845 \pm 0.022 \pm 0.033$ & $4500-5000$ & $01403+00008+00060$ \\
\hline $0.325-0.350$ & $3.055 \pm 0.019 \pm 0.030$ & $\begin{array}{l}4.000-3.000 \\
5.000-5500\end{array}$ & $\begin{array}{l}0.1403 \pm 0.0008 \text { 土 } 0.0009 \\
0.096+0009+0\end{array}$ \\
\hline $0.350-0.375$ & $2.476 \pm 0.018 \pm 0.034$ & $3.000-3.000$ & $0.0280 \pm 0.0000 \pm 0.0021$ \\
\hline $0.375-0.400$ & $1.978 \pm 0.016 \pm 0.039$ & $5.500-6.000$ & $0.0039 \pm 0.0001 \pm 0.0006$ \\
\hline $0.400-0.430$ & $1.550 \pm 0.013 \pm 0.041$ & & \\
\hline $0.430-0.460$ & $1.212 \pm 0.011 \pm 0.036$ & & \\
\hline $0.460-0.490$ & $0.9375 \pm 0.0098 \pm 0.0273$ & & \\
\hline $0.490-0.520$ & $0.7318 \pm 0.0086 \pm 0.0190$ & & \\
\hline $0.520-0.550$ & $0.5672 \pm 0.0077 \pm 0.0139$ & & \\
\hline $0.550-0.600$ & $0.4022 \pm 0.0049 \pm 0.0098$ & & \\
\hline $0.600-0.650$ & $0.2602 \pm 0.0040 \pm 0.0064$ & & \\
\hline $0.650-0.700$ & $0.1721 \pm 0.0033 \pm 0.0044$ & & \\
\hline $0.700-0.750$ & $0.1064 \pm 0.0025 \pm 0.0029$ & & \\
\hline $0.750-0.800$ & $0.0587 \pm 0.0017 \pm 0.0020$ & & \\
\hline $0.800-0.900$ & $0.0262 \pm 0.0008 \pm 0.0015$ & & \\
\hline $0.900-1.000$ & $0.0047 \pm 0.0003 \pm 0.0009$ & & \\
\hline
\end{tabular}

Table 5: Distributions of $x_{p}=p / p_{\text {beam }}$ and rapidity $y$. 


\begin{tabular}{|c|c|c|c|}
\hline Interval & $(1 / N)\left(d N / d p_{\perp}^{\text {in }}\right) \pm$ stat. \pm sys. & & \\
\hline $0.000-0.100$ & $48.30 \pm 0.05 \pm 0.29$ & & \\
\hline $0.100-0.200$ & $38.14 \pm 0.04 \pm 0.43$ & & \\
\hline $0.200-0.300$ & $28.21 \pm 0.03 \pm 0.15$ & Interval & $(1 / N)\left(d N / d p_{\perp}^{\text {out }}\right) \pm$ stat. \pm sys. \\
\hline $0.300-0.400$ & $20.45 \pm 0.03 \pm 0.08$ & $0.000-0.100$ & $67.66 \pm 0.05 \pm 0.37$ \\
\hline $0.400-0.500$ & $15.05 \pm 0.02 \pm 0.05$ & $0.100-0.200$ & $51.30 \pm 0.04 \pm 0.56$ \\
\hline $0.500-0.600$ & $11.22 \pm 0.02 \pm 0.04$ & $0.200-0.300$ & $34.35 \pm 0.03 \pm 0.17$ \\
\hline $0.600-0.700$ & $8.522 \pm 0.017 \pm 0.041$ & $0.300-0.400$ & $21.40 \pm 0.03 \pm 0.09$ \\
\hline $0.700-0.800$ & $6.602 \pm 0.015 \pm 0.034$ & $0.400-0.500$ & $12.86 \pm 0.02 \pm 0.05$ \\
\hline $0.800-0.900$ & $5.180 \pm 0.013 \pm 0.026$ & $0.500-0.600$ & $7.722 \pm 0.017 \pm 0.029$ \\
\hline $0.900-1.000$ & $4.156 \pm 0.012 \pm 0.021$ & $0.600-0.700$ & $4.690 \pm 0.013 \pm 0.018$ \\
\hline $1.000-1.200$ & $3.045 \pm 0.007 \pm 0.016$ & $0.700-0.800$ & $2.881 \pm 0.010 \pm 0.012$ \\
\hline $1.200-1.400$ & $2.095 \pm 0.006 \pm 0.012$ & $0.800-0.900$ & $1.830 \pm 0.008 \pm 0.011$ \\
\hline $1.400-1.600$ & $1.473 \pm 0.005 \pm 0.010$ & $0.900-1.000$ & $1.198 \pm 0.007 \pm 0.009$ \\
\hline $1.600-1.800$ & $1.081 \pm 0.004 \pm 0.008$ & $1.000-1.200$ & $0.6731 \pm 0.0040 \pm 0.0061$ \\
\hline $1.800-2.000$ & $0.8036 \pm 0.0038 \pm 0.0075$ & $1.200-1.400$ & $0.3265 \pm 0.0028 \pm 0.0033$ \\
\hline $2.000-2.500$ & $0.5104 \pm 0.0021 \pm 0.0051$ & $1.400-1.600$ & $0.1687 \pm 0.0020 \pm 0.0029$ \\
\hline $2.500-3.000$ & $0.2753 \pm 0.0015 \pm 0.0029$ & $1.600-1.800$ & $0.0922 \pm 0.0015 \pm 0.0026$ \\
\hline $3.000-3.500$ & $0.1601 \pm 0.0011 \pm 0.0021$ & $1.800-2.000$ & $0.0525 \pm 0.0011 \pm 0.0020$ \\
\hline $3.500-4.000$ & $0.0970 \pm 0.0008 \pm 0.0015$ & $2.000-2.500$ & $0.0223 \pm 0.0005 \pm 0.0010$ \\
\hline $4.000-5.000$ & $0.0493 \pm 0.0004 \pm 0.0008$ & $2.500-3.000$ & $0.0069 \pm 0.0003 \pm 0.0004$ \\
\hline $5.000-6.000$ & $0.0202 \pm 0.0003 \pm 0.0004$ & $3.000-3.500$ & $0.0026 \pm 0.0002 \pm 0.0002$ \\
\hline $6.000-7.000$ & $0.0088 \pm 0.0002 \pm 0.0002$ & $3.500-5.000$ & $0.00040 \pm 0.00004 \pm 0.00004$ \\
\hline $7.000-8.000$ & $0.0040 \pm 0.0001 \pm 0.0002$ & & \\
\hline $8.000-10.000$ & $0.00124 \pm 0.00005 \pm 0.00007$ & & \\
\hline $10.000-14.000$ & $0.00015 \pm 0.00001 \pm 0.00003$ & & \\
\hline
\end{tabular}

Table 6: Distributions of $p_{\perp}^{\text {in }}$ and $p_{\perp}^{\text {out }}$. 


\subsubsection{Description of the Models}

\section{Lund Parton Shower Model (JETSET)}

In the Lund Parton Shower (PS) model (program JETSET version 7.4 [25, 29]), the evolution of the parton system is treated as a branching process based on the leading logarithm approximation (LLA). In this picture partons undergo decays of the type $q \rightarrow q g, g \rightarrow g g$ and $g \rightarrow q \bar{q}$. The probability for the decay of a parton $a$ with virtual mass $m_{a}$ into partons $b$ and $c$ is given by the Altarelli-Parisi ("DGLAP") equation (see, e.g., [13]),

$$
\frac{d \mathcal{P}_{a \rightarrow b c}}{d t}=\int d z \frac{\alpha_{s}\left(Q^{2}\right)}{2 \pi} P_{a \rightarrow b c}(z)
$$

where the evolution parameter $t$ is related to the parent's virtual mass and to the QCD scale parameter $\Lambda$ by $t=\ln \left(m_{a}^{2} / \Lambda^{2}\right)$. The strong coupling constant $\alpha_{s}\left(Q^{2}\right)$ is evaluated at $Q^{2}$ equal to the transverse momentum squared of the branching. $P_{a \rightarrow b c}(z)$ is the Altarelli-Parisi splitting function. This function is also used to generate the energy fractions $z$ and $1-z$ carried by the daughters. The decay angle is determined by two-body kinematics once the parent and daughter masses and energy fractions have been fixed.

For the first branchings of the initial quark and antiquark, an acceptance-rejection technique is applied so as to reproduce the $O\left(\alpha_{s}\right)$ three-jet cross section. Coherence effects are included by requiring that the emission angles of successive branchings always decrease (angular ordering). Certain other higher order effects are also included, such as the azimuthal distribution in gluon decays from spin and coherence effects. The parton shower is stopped when the parton virtualities drop below a cut-off, $M_{\min }$ (for parameter values see Table 8).

The conversion of the partons into hadrons is accomplished with the Lund String Model [29]. Gluons are associated with momentum carrying kinks in the string. Hadron production results from a breaking of the string which can be interpreted as virtual quark-antiquark pair production in a flux-tube. The quarks' (equal and opposite) transverse momenta are generated according to a Gaussian distribution of width $\sigma_{q}$. Longitudinal hadron momenta are determined by means of phenomenological fragmentation functions: the Lund symmetric function with parameters $a$ and $b$ for light $(u, d, s)$ quarks, and the Peterson function [32] with parameters $\epsilon_{c}$ and $\epsilon_{b}$ for $c$ and $b$ quarks.

The probabilities for $u \bar{u}$ and $d \bar{d}$ production within the string are assumed to be equal, and the probability for $s \bar{s}$ is left as a free parameter, called in the following $s / u$ (in the JETSET program PARJ (2)). Baryon production is included by allowing diquark-antiquark pairs to be created; the probability for this to occur is given by the parameter $q q / q$ (PARJ (1)). Meson production in the string between the baryon and antibaryon (the "popcorn" mechanism) is also allowed, and occurs with a relative frequency given by the parameter PARJ $(5)=0.5$ (its default value). The popcorn mechanism is investigated further in Section 5.3.

To describe baryons at high momenta it has been found necessary to activate the parameter PARJ (19) (LBS, leading baryon suppression factor) which suppresses diquark production in the breakup closest to the endpoint of the string. Without this parameter, the Lund symmetric fragmentation function would predict an increase in the proton fraction at high momenta, which is contrary to observation (see Section 5.2, Fig. 64(b)).

The production rates of $L=1$ mesons, $(S=0, J=1)$ and $(S=1, J=0,1,2)$, are controlled by the four parameters PARJ (14-17). In the fitting procedure described below, only the $J^{P}=2^{+}$rate is taken as a free parameter and the other rates are related by invoking spin 
counting in the ratio $0^{+}: 1^{+}: 2^{+}=1: 3: 5$. In addition, $\operatorname{PARJ}(14)=\operatorname{PARJ}(16)$ is assumed, i.e., the rate of $(S=0, J=1)$ is set equal to the rate of $(S=1, J=1)$.

Ideal mixing is used for the flavour-diagonal neutral mesons with the exception of the pseudoscalar nonet, where a mixing angle of $9.7^{\circ}$ is taken. Bose-Einstein correlations are not included in the fit performed here.

\section{HERWIG}

The HERWIG Monte Carlo (version 5.8) [31] is also based on a parton branching process, as described for JETSET. Instead of the parton virtual mass for the evolution parameter, HERWIG uses $t=\ln \left(\zeta_{a}^{2} / \Lambda^{2}\right)$, where $\zeta_{a}$ is defined by

$$
\begin{aligned}
& \zeta_{a}=E_{a} \sqrt{\xi_{b c}}, \\
& \xi_{b c}=\frac{p_{b} \cdot p_{c}}{E_{b} E_{c}},
\end{aligned}
$$

for the branching $a \rightarrow b c$ where $p_{b}, p_{c}, E_{b}$ and $E_{c}$ are the four-momenta and energies of partons $b$ and $c$. Angular ordering of successive branchings is approximately equivalent to ordering of the $\xi_{b c}$. The argument $z$ of the Altarelli-Parisi splitting functions is taken to be the daughter's energy fraction, and the scale for $\alpha_{s}$ is the transverse momentum squared of the branching. Azimuthal asymmetries for gluon decays both from coherence and spin effects are included. The treatment of hard gluon emission is improved by matching the parton shower cross section to the $O\left(\alpha_{s}\right)$ matrix element.

The hadronization in HERWIG is modelled with a cluster mechanism. At the end of the parton shower, all gluons split into quark-antiquark pairs. Neighboring $q \bar{q}$ pairs form colourneutral clusters which (usually) decay into two hadrons. Special treatment is given to very light clusters, which are allowed to "decay" into a single hadron, and to very heavy clusters ( aass $>M_{c l, \max }$ ) which can decay further into clusters before decaying into hadrons. Baryons are produced from cluster decays into baryon-antibaryon pairs, i.e. clusters themselves always have baryon number of zero. If a cluster contains a quark that originated in the perturbative phase of the parton shower (i.e. not from the non-perturbative gluon splitting) then the angular distribution for the hadron that contains this quark is given by an exponential distribution in $1-\cos \theta$ peaked in the quark's direction, with mean value given by the parameter CLSMR.

The global event-shape and hadron momentum spectrum are largely determined by the parameters, $\Lambda$ and $Q_{0}$, governing the parton shower, and to a lesser extent by the thresholds for clusters of too high or too low mass. The hadron flavour composition is mainly determined by the available phase space in cluster decay (i.e. by the cluster mass spectrum), and also by an additional parameter PWT(3) which gives the probability for $s \bar{s}$ production in cluster decay.

\section{ARIADNE}

Instead of formulating the perturbative QCD cascade in terms of quark and gluon decays, the ARIADNE Monte Carlo (version 4.08) [30] uses the complementary language of colour dipoles [33]. In this approach, the initially produced colour dipole (the $q \bar{q}$ pair) radiates a gluon according to the first order QCD matrix element. The resulting $q \bar{q} g$ system is then treated as two independent dipoles, between the quark and gluon, and between the gluon and antiquark. Each successive gluon emission creates a new dipole, all of which are assumed to radiate independently. This approach naturally takes into account coherence effects, azimuthal 
dependence of gluon decays, and an exact $O\left(\alpha_{s}\right)$ description of hard gluon radiation which must be inserted by hand into the parton cascade approach used in JETSET and HERWIG.

The free parameters in the perturbative phase of ARIADNE are the QCD scale $\Lambda$ and the minimum transverse momentum allowed in dipole emission $p_{\perp}^{\min }$. The non-perturbative hadron production in ARIADNE is accomplished with the Lund String Model as described for JETSET.

\subsubsection{Fitting of Model Parameters}

The determination of model parameters is based on comparison with the measured event-shape and inclusive distributions presented in Section 2, as well as with distributions of identified hadrons given in Section 5.2. Specifically, the following quantities were used:

- event-shape distributions $S, A, 1-T, T_{\text {minor }},-\ln y_{3}$;

- charged particle inclusive distributions $x_{p}=p / p_{\text {beam }}, p_{\perp}^{\text {out }}, p_{\perp}^{\text {in }}$;

- inclusive spectra of $\xi=-\ln x_{p}$ for the neutral $V^{0}$ particles $K^{0}$ and $\Lambda^{0}$, and for the charged pions, kaons and (anti)protons;

- inclusive spectra of $x=E / E_{\text {beam }}$ for the mesons $\eta(x>0.1), \eta^{\prime}(x>0.1) \rho^{0}, K^{* 0}, K^{*+}$, $\phi^{0}, \omega^{0}$

- the mean multiplicities of the $L=1$ mesons $f_{2}, f_{0}$ and of the hyperons $\Xi^{-}, \Sigma(1385)$, $\Xi(1530)^{0}, \Omega^{-}$. Multiplicities for the $f_{0}$ and $f_{2}$ mesons are taken from [34].

The dependence of measurable quantities on the model parameters does not exist in analytical form. Instead, Monte Carlo calculations of these quantities at various locations in parameter space have to be performed, and a parametrization for the dependence of each bin on the model parameters is determined. It is reasonable to assume, and has been confirmed in practice, that the dependence is smooth, though not necessarily linear.

The method used in the previous ALEPH publication [26] was based on 2nd order polynomials to parametrize the distributions. The fast (quadratic) rise of computer time with the number of parameters limited the practical application to $n \leq 6$ parameters. Since then, the increasing accuracy of the experimental data and the demands of the physics analyses made it desirable to fit many more parameters. The prominent example is the large number of JETSET string model parameters. Therefore it was necessary to go to a linear approach.

A measurable quantity (e.g. the contents of interval $j$ of a distribution, or the mean multiplicity of a given particle) will be denoted by $M_{j}$ and the set of model parameters to be determined by $x_{i}, i=1, \ldots, n$. Starting with a given set of initial values $x_{i 0}$, a set of $4 N$ Monte Carlo events is generated (typically $N=1$ million) and the histograms of the various distributions are filled. For each parameter, four more points in the parameter space are considered, situated at distances

$$
-\Delta x_{l e f t},-\Delta x_{l e f t} / 2, \Delta x_{\text {right }} / 2, \Delta x_{\text {right }},
$$

from the initial value $x_{i 0}$. At each of these four points, and for each parameter, $N$ Monte Carlo events are generated and the histograms stored. 
As an option, the four points can all be set to the left or right side of $x_{i 0}$, which is important in case a parameter has to obey a physical constraint. An example is the cut-off mass of the parton shower which is not allowed to be smaller than $\Lambda_{Q C D}$.

A linear expression

$$
M_{j}\left(x_{i}\right)=m_{j}^{i}+a_{j}^{i}\left(x_{i}-x_{i 0}\right)
$$

is fitted for each measured quantity $M_{j}$ and each parameter $x_{i}$, yielding a slope $a_{j}^{i}$ and offset $m_{j}^{i}$. If the fit is not acceptable ( $\chi^{2}$ probability less than $10 \%$ ) a quadratic expression is tried and used to monitor the parameter region for which the linear expression is acceptable. The actual parameter fit is always performed using the linear terms only. The entire process of Monte Carlo event generation is then repeated, moving the central values $x_{i 0}$ accordingly, until the final fitted values of the parameters all remain within the linear ranges and change by less than their errors.

The model parameters are fitted by minimizing the $\chi^{2}$ function

$$
\chi^{2}=\sum_{j}\left(\frac{M_{j}^{\text {data }}-M_{j}^{M C}(\vec{x})}{\sigma_{j}^{\text {data }}}\right)^{2}
$$

with respect to the parameters $\vec{x}$, where the sum runs over all of the measured quantities. The errors $\sigma_{j}^{\text {data }}$ are taken to be the quadratic sums of statistical and systematic uncertainties; Monte Carlo errors are small compared to these and are neglected.

By using Eq. (25) it is implicitly assumed that the experimental data points are uncorrelated. This is not true in general, however, since there are both intrinsic correlations between variables as well as systematic errors from the correction procedure which introduce correlations between neighboring bins. As a result, the errors on the fitted parameters and the corresponding $\chi^{2}$ values should only be regarded as giving a rough measure of statistical uncertainty and relative goodness-of-fit.

As has been observed previously [26], model predictions for the rate of particle production transverse to the event plane for $p_{\perp}^{\text {out }}$ greater than about $1 \mathrm{GeV}$ are low by up to $30 \%$. A correct description of this region seems outside the capabilities of current QCD parton shower models. Therefore the regions $p_{\perp}^{\text {out }} \geq 0.7 \mathrm{GeV}, A \geq 0.06$ and $T_{\text {minor }} \geq 0.2$ are excluded from the fit. As a result the QCD scale parameter of JETSET $\Lambda_{Q C D}$ is reduced by about $25 \mathrm{MeV}$, and the multijet regions of the sphericity, thrust, and $y_{3}$ distributions are better described.

Because of the large number of parameters (15) in JETSET and ARIADNE, the following special treatment is necessary. First, the parameters $\epsilon_{c}$ and $\epsilon_{b}$ controlling the fragmentation of hadrons containing $c$ and $b$ quarks are adjusted so as to describe the corresponding measured $\langle x\rangle$ values $[35,36,37]$. The probability for a meson containing a $c$ or $b$ quark to have spin 1 is set to 0.65 , which represents a compromise between the values of $0.55(0.75)$ necessary to describe charm (bottom) hadrons separately [35, 38]. The heavy vector meson probability of 0.65 together with the tensor meson parameter PARJ (17) $=0.2$ provides a good description of the measured fraction of $B^{* *} / B$ [38]. The parameter PARJ(17) is held fixed at 0.20 in the following since it also gives a good description of light tensor meson $\left(f_{2}\right)$ production. Furthermore, the parameter $a$ is fixed to 0.4 , since it is found to be highly correlated with the parameter $b$.

The remaining JETSET and ARIADNE parameters are then separated into the following two groups:

- the general fragmentation parameters: $\Lambda_{Q C D}, M_{m i n}, \sigma_{q}, \mathrm{~b}$; 
- the spin/flavour parameters controlling the type of hadron produced: $P(S=1)_{u, d}$, $P(S=1)_{s}, s / u, q q / q,(s u / d u) /(s / u), \eta^{\prime}$-suppression factor (PARJ (26)), leading-baryon suppression factor (PARJ (19)).

First, a global fit of these 11 parameters is performed. This yields starting values for the next step, where only the spin/flavour parameters are fitted to the identified particle data, and the general fragmentation parameters are held fixed. Then, the spin/flavour parameters are held fixed and the fragmentation parameters fitted to the charged particle data.

Because of the smaller number of parameters in the HERWIG model (five), the multi-step procedure described above is not necessary, and a global fit of the parameters was performed. In this case, the region $p_{\perp}^{\text {out }} \geq 0.7 \mathrm{GeV}$, was excluded from the fit. One characteristic feature of HERWIG is the extreme sensitivity of baryon production to the parameter $M_{c l, m a x}$, growing with the mass of the baryon considered. This parameter is also constrained by the charged particle $p_{\perp}$ distributions. It was found impossible to reproduce the rates of all measured baryon species with one set of parameter values. Therefore in the global fit to determine the 5 HERWIG parameters, the baryons other than proton and $\Lambda^{0}$ were excluded, and only the mean $\Lambda^{0}$ multiplicity was used instead of the momentum spectrum.

The fitted parameter values are shown in Tables 8 through 10 below. Systematic uncertainties in the parameters have been estimated by varying the choice of the set of distributions and the fit regions used. From Figs. 5 through 16 in Section 2, one sees that the overall description of the data by the QCD models is quite reasonable, though by no means perfect. The discrepancies are often significantly larger than one standard deviation, and as a result the errors given for the fitted parameters do not reflect the actual level of discrepancy between data and model prediction.

\subsubsection{Discussion of the Results}

The event-shape distributions $S$ and $1-T$ are well reproduced by JETSET and ARIADNE, while HERWIG gives a somewhat worse fit in particular at high values of $1-T$. The predictions of JETSET and ARIADNE are systematically below the data at high values of $A$ and $T_{\text {minor }}$, which are the variables related to the particle momenta perpendicular to the event plane. While HERWIG is much better in this region, it shows problems in the peak regions of these variables.

The $n$-jet rates for $n=2,3,4,5$ are shown in Fig. 17, and the values are given in Table 7 . They have not been used in the model fits. Here the rates correspond to both charged and neutral particles (measured using energy-flow objects; cf. Section 1.2.6). The three-jet rate predicted by JETSET is significantly higher than the measurement and the two-jet rate is predicted too low. HERWIG and ARIADNE describe the data much better.

The scaled momentum $\left(x_{p}\right)$ distribution, which decreases by 5 orders of magnitude from low to high $x_{p}$, is seen to be better described by JETSET or ARIADNE than by HERWIG. All model predictions are low in the region of very low momenta $\left(x_{p}<0.014\right)$. This feature is more clearly seen in the distribution of $\xi=-\ln x_{p}$ (cf. Section 4.1.1). The discrepancy is clearly related to the charged pions (see Fig. 63 in Section 5.2). Some deviations are seen for JETSET and ARIADNE at intermediate $x_{p}$ values $(0.2-0.3)$ and at the high end of the spectrum $\left(x_{p}>0.75\right)$. This latter region is very sensitive to the values of the fragmentation parameters.

All models exhibit a major problem in the $p_{\perp}^{\text {out }}$ distribution above $800 \mathrm{MeV}$. The discrepancy reaches $35 \%$ at the highest values. This presumably stems from inaccuracies of the leading-log 




Figure 17: The $n$-jet rates based on the Durham algorithm.

\begin{tabular}{|c|cccc|}
\hline$y_{\text {cut }}$ & $R_{2}$ & $R_{3}$ & $R_{4}$ & $R_{5}$ \\
\hline 0.00100 & $0.1252 \pm 0.0030$ & $0.2837 \pm 0.0020$ & $0.2986 \pm 0.0016$ & $0.1837 \pm 0.0025$ \\
0.00126 & $0.1717 \pm 0.0035$ & $0.3266 \pm 0.0026$ & $0.2867 \pm 0.0017$ & $0.1479 \pm 0.0023$ \\
0.00158 & $0.2249 \pm 0.0035$ & $0.3598 \pm 0.0033$ & $0.2635 \pm 0.0032$ & $0.1130 \pm 0.0023$ \\
0.00200 & $0.2821 \pm 0.0032$ & $0.3812 \pm 0.0039$ & $0.2335 \pm 0.0033$ & $0.0819 \pm 0.0033$ \\
0.00251 & $0.3407 \pm 0.0029$ & $0.3918 \pm 0.0042$ & $0.1993 \pm 0.0034$ & $0.0575 \pm 0.0045$ \\
0.00316 & $0.3977 \pm 0.0027$ & $0.3917 \pm 0.0036$ & $0.1660 \pm 0.0025$ & $0.0394 \pm 0.0048$ \\
0.00398 & $0.4523 \pm 0.0033$ & $0.3837 \pm 0.0030$ & $0.1356 \pm 0.0022$ & $0.0260 \pm 0.0050$ \\
0.00501 & $0.5036 \pm 0.0035$ & $0.3696 \pm 0.0029$ & $0.1094 \pm 0.0015$ & $0.0165 \pm 0.0049$ \\
0.00631 & $0.5512 \pm 0.0030$ & $0.3517 \pm 0.0031$ & $0.0867 \pm 0.0011$ & $0.0100 \pm 0.0043$ \\
0.00794 & $0.5962 \pm 0.0026$ & $0.3302 \pm 0.0027$ & $0.0674 \pm 0.0009$ & $0.0058 \pm 0.0033$ \\
0.01000 & $0.6395 \pm 0.0027$ & $0.3063 \pm 0.0029$ & $0.0509 \pm 0.0009$ & $0.0032 \pm 0.0023$ \\
0.01259 & $0.6803 \pm 0.0028$ & $0.2805 \pm 0.0032$ & $0.0372 \pm 0.0005$ & $0.0018 \pm 0.0014$ \\
0.01585 & $0.7195 \pm 0.0029$ & $0.2532 \pm 0.0032$ & $0.0262 \pm 0.0005$ & $0.0008 \pm 0.0007$ \\
0.01995 & $0.7562 \pm 0.0025$ & $0.2256 \pm 0.0027$ & $0.0176 \pm 0.0004$ & $0.0004 \pm 0.0003$ \\
0.02512 & $0.7906 \pm 0.0022$ & $0.1975 \pm 0.0022$ & $0.0114 \pm 0.0003$ & $0.0002 \pm 0.0001$ \\
0.03162 & $0.8231 \pm 0.0020$ & $0.1697 \pm 0.0020$ & $0.0068 \pm 0.0003$ & \\
0.03981 & $0.8534 \pm 0.0016$ & $0.1427 \pm 0.0016$ & $0.0036 \pm 0.0002$ & \\
0.05012 & $0.8811 \pm 0.0013$ & $0.1168 \pm 0.0014$ & $0.0018 \pm 0.0001$ & \\
0.06310 & $0.9067 \pm 0.0011$ & $0.0923 \pm 0.0008$ & $0.0007 \pm 0.0001$ & \\
0.07943 & $0.9295 \pm 0.0011$ & $0.0701 \pm 0.0009$ & $0.0002 \pm 0.0001$ & \\
0.10000 & $0.9488 \pm 0.0006$ & $0.0509 \pm 0.0004$ & & \\
\hline
\end{tabular}

Table 7: Measured values of the $n$-jet rates using the Durham cluster algorithm as a function of the jet-resolution parameter $y_{c u t}$. 
approximation on which the parton shower models are based. The $p_{\perp}^{\text {in }}$ distribution is not very well described either. The model predictions are low at high values $(>4 \mathrm{GeV})$ and exhibit a wave structure around the data below about $2 \mathrm{GeV}$.

The comparison of data and model predictions for the production of identified hadrons is discussed in Section 5.2.

\begin{tabular}{|c|c|c|c|c|c|c|}
\hline parameter & $\begin{array}{l}\text { name in } \\
\text { program }\end{array}$ & $\begin{array}{c}\text { default } \\
\text { value }\end{array}$ & $\begin{array}{c}\text { range } \\
\text { generated }\end{array}$ & value & $\begin{array}{l}\text { fit result } \\
\text { error }\end{array}$ & syst. \\
\hline$\overline{\Lambda_{Q C D}(\mathrm{GeV})}$ & PARJ(81) & 0.29 & $0.21-0.37$ & 0.292 & \pm 0.003 & \pm 0.006 \\
\hline$M_{\min }(\mathrm{GeV})$ & PARJ(82) & 1.0 & $1.0-2.0$ & 1.57 & \pm 0.04 & \pm 0.13 \\
\hline$\sigma_{q}(\mathrm{GeV})$ & PARJ(21) & 0.36 & $0.28-0.44$ & 0.370 & \pm 0.002 & \pm 0.008 \\
\hline$a$ & $\operatorname{PARJ}(41)$ & 0.30 & $0.20-0.60$ & 0.40 & (fixed) & \\
\hline$b\left(\mathrm{GeV}^{-2}\right)$ & $\operatorname{PARJ}(42)$ & 0.58 & $0.60-1.00$ & 0.796 & \pm 0.012 & \pm 0.033 \\
\hline$\epsilon_{c}$ & $-\operatorname{PARJ}(54)$ & 0.050 & $0.015-0.065$ & 0.040 & adjusted & \\
\hline$\epsilon_{b}$ & -PARJ(55) & 0.005 & $0.0005-0.0075$ & 0.0035 & adjusted & \\
\hline$p(S=1)_{d, u}$ & PARJ(11) & 0.50 & $0.40-0.70$ & 0.5 .5 & \pm 0.02 & \pm 0.06 \\
\hline$p(S=1)_{s}$ & PARJ(12) & 0.60 & $0.35-0.65$ & 0.47 & \pm 0.02 & \pm 0.06 \\
\hline$p(S=1)_{c, b}$ & PARJ(13) & 0.75 & $0.50-0.80$ & 0.65 & adjusted & \\
\hline$p\left(J^{P}=2^{+} ; L=1, S=1\right)$ & PARJ(17) & 0.0 & $0.10-0.30$ & 0.20 & adjusted & \\
\hline extra $\eta^{\prime}$ suppression & PARJ(26) & 0.40 & $0.05-0.55$ & 0.27 & \pm 0.03 & \pm 0.09 \\
\hline$s / u$ & PARJ( 2) & 0.30 & $0.19-0.39$ & 0.285 & \pm 0.004 & \pm 0.014 \\
\hline$q q / q$ & PARJ( 1) & 0.10 & $0.05-0.15$ & 0.106 & \pm 0.002 & \pm 0.003 \\
\hline$(s u / d u) /(s / u)$ & PARJ( 3$)$ & 0.40 & $0.4-1.0$ & 0.71 & \pm 0.04 & \pm 0.07 \\
\hline leading baryon suppr. & PARJ(19) & 1.0 & $0.2-1.0$ & 0.57 & \pm 0.03 & \pm 0.10 \\
\hline switch & & & & setting & & \\
\hline fragmentation function & MSTJ(11) & 4 & & 3 & & \\
\hline baryon model & MSTJ(12) & 2 & & 3 & & \\
\hline azimuthal distrib. in PS & $\operatorname{MSTJ}(46)$ & 3 & & 3 & & \\
\hline
\end{tabular}

Table 8: Parameters for JETSET 7.4. The parameters describing the higher mesons are assumed to be in the ratio PARJ (17):PARJ(16):PARJ(15) $=5: 3: 1$, and PARJ(14)=PARJ(16). The diquarkspin suppression parameter PARJ (4) was left at its default value (0.05). No Bose-Einstein correlations are included.

\begin{tabular}{|c|c|c|c|ccc|}
\hline parameter & $\begin{array}{c}\text { name in } \\
\text { program }\end{array}$ & $\begin{array}{c}\text { default } \\
\text { value }\end{array}$ & $\begin{array}{c}\text { range } \\
\text { generated }\end{array}$ & \multicolumn{3}{|c|}{ fit result } \\
value & error & syst. \\
\hline$\Lambda_{Q C D}(\mathrm{GeV})$ & QCDLAM & 0.18 & $0.12-0.18$ & 0.147 & \pm 0.001 & \pm 0.005 \\
$M_{\text {gluon }}(\mathrm{GeV})$ & RMASS $(13)$ & 0.75 & $0.7-1.0$ & 0.656 & \pm 0.005 & \pm 0.015 \\
$M_{c l, \max }(\mathrm{GeV})$ & CLMAX & 3.35 & $3.0-4.0$ & 3.65 & \pm 0.01 & \pm 0.10 \\
$s(\theta)$ & CLSMR & 0.0 & $0.0-1.0$ & 0.73 & \pm 0.02 & \pm 0.06 \\
p(s-quark $)$ & PWT $(3)$ & 1.0 & $0.6-1.0$ & 0.79 & \pm 0.01 & \pm 0.06 \\
\hline
\end{tabular}

Table 9: Parameters for HERWIG 5.8. 


\begin{tabular}{|c|c|c|c|c|c|c|}
\hline parameter & $\begin{array}{l}\text { name in } \\
\text { program }\end{array}$ & $\begin{array}{c}\text { default } \\
\text { value }\end{array}$ & $\begin{array}{c}\text { range } \\
\text { generated }\end{array}$ & value & $\begin{array}{l}\text { fit result } \\
\text { error }\end{array}$ & syst. \\
\hline$\Lambda_{Q C D}(\mathrm{GeV})$ & $\operatorname{PARA}(1)$ & 0.22 & $0.15-0.30$ & 0.230 & \pm 0.002 & \pm 0.005 \\
\hline$p_{T, \min }(\mathrm{GeV})$ & $\operatorname{PARA}(3)$ & 0.60 & $0.6-1.1$ & 0.79 & \pm 0.03 & \pm 0.04 \\
\hline$\sigma_{q}(\mathrm{GeV})$ & PARJ(21) & 0.36 & $0.28-0.44$ & 0.358 & \pm 0.002 & \pm 0.010 \\
\hline & $\operatorname{PARJ}(41)$ & 0.30 & $0.20-0.60$ & 0.40 & (fixed) & \\
\hline$b\left(\mathrm{GeV}^{-2}\right)$ & PARJ(42) & 0.58 & $0.65-1.05$ & 0.823 & \pm 0.015 & \pm 0.05 \\
\hline$\epsilon_{c}$ & $-\operatorname{PARJ}(54)$ & 0.050 & $0.025-0.075$ & 0.040 & adjusted & \\
\hline$\epsilon_{b}$ & -PARJ(55) & 0.005 & $0.0005-0.0085$ & 0.0035 & adjusted & \\
\hline$p(S=1)_{d, u}$ & PARJ(11) & 0.50 & $0.35-0.65$ & 0.57 & \pm 0.02 & \pm 0.03 \\
\hline$p(S=1)_{s}$ & PARJ(12) & 0.60 & $0.35-0.65$ & 0.47 & \pm 0.02 & \pm 0.04 \\
\hline$p(S=1)_{c, b}$ & PARJ(13) & 0.75 & $0.50-0.80$ & 0.65 & adjusted & \\
\hline$p\left(J^{P}=2^{+} ; L=1, S=1\right)$ & PARJ(17) & 0.0 & $0.07-0.27$ & 0.20 & adjusted & \\
\hline extra $\eta^{\prime}$ suppression & PARJ(26) & 0.40 & $0.10-0.60$ & 0.29 & \pm 0.03 & \pm 0.02 \\
\hline$s / u$ & PARJ( 2) & 0.30 & $0.19-0.39$ & 0.286 & \pm 0.004 & \pm 0.017 \\
\hline$q q / q$ & PARJ( 1) & 0.10 & $0.05-0.15$ & 0.115 & \pm 0.003 & \pm 0.003 \\
\hline$(s u / d u) /(s / u)$ & PARJ( 3) & 0.40 & $0.2-0.9$ & 0.65 & \pm 0.05 & \pm 0.07 \\
\hline leading baryon suppr. & PARJ(19) & 1.0 & $0.2-1.0$ & 0.52 & \pm 0.03 & \pm 0.10 \\
\hline switch & & & & setting & & \\
\hline fragmentation function & MSTJ(11) & 4 & & 3 & & \\
\hline baryon model & MSTJ(12) & 2 & & 3 & & \\
\hline
\end{tabular}

Table 10: Parameters for ARIADNE 4.08 (using JETSET 7.4 for hadronization). The parameters describing the higher mesons are assumed to be in the ratio PARJ (17):PARJ(16):PARJ(15) $=5: 3: 1$, and PARJ (14) = PARJ(16). The diquark-spin suppression parameter PARJ(4) was left at its default value (0.05). No Bose-Einstein correlations are included. 


\section{Hard QCD}

In this section attention is given primarily to the basic components of QCD. First, the spin properties of the fundamental fields, quarks and gluons, are measured. Although this has been done years ago in experiments at lower centre-of-mass energies, it is still interesting to see with LEP data, which, because of the smaller non-perturbative effects, exhibit jet configurations that resemble more closely the underlying parton structure of the events.

A large fraction of the section is devoted to the measurement of the only free parameter of QCD, $\alpha_{s}$. Different methods have been used and are presented in Section 3.2. Also a compilation of $\alpha_{s}$ determinations at different energies and accelerators is shown, and the running of $\alpha_{s}$ with the scale is established from them. A study of the interplay between event shape and event orientation is presented, and the independence of the strong coupling constant from the quark flavour, as predicted in QCD, is checked using b-quark enriched samples.

Finally, the structure of the gauge group on which QCD is based, SU(3), can be probed, because the QCD predictions depend on the structure constants of the group. Two-, three- and four-jet events are used to measure these constants and exclude other possible gauge groups for the strong interactions.

\subsection{Parton Spins}

It is a well established fact that quarks are spin-1/2 particles [39] and gluons are spin-1 particles [40]. It is instructive nevertheless to look at the experimental data collected at LEP in order to assess the sensitivity of the measurements to the spin assignment for the building blocks of the nucleon.

\subsubsection{Quark Spin}

The quark spin can be inferred from the angular distribution of the thrust axis in hadronic $\mathrm{Z}$ decays. This axis is a rather good approximation for the direction of the primary quarks produced in the process $e^{+} e^{-} \rightarrow q \bar{q} \rightarrow$ hadrons. Since one cannot distinguish $q$ and $\bar{q}$, the angle $\Theta$ between the incoming beam and the direction of the final state quarks is always taken in the range $0 \leq \Theta \leq 90^{\circ}$. From the fact that the $Z$ has spin 1 and ignoring the masses of initial and final state particles, the angular distribution can be determined from simple angular momentum arguments. One obtains

$$
\frac{d \sigma}{d \cos \Theta} \propto 1+\cos ^{2} \Theta \text { and } \frac{d \sigma}{d \cos \Theta} \propto 1-\cos ^{2} \Theta
$$

for spin-1/2 and scalar quarks, respectively. By measuring the direction of the thrust axis, i.e. $\Theta_{\text {THRUST }}$ instead of $\Theta$, these simple predictions are modified by higher order QCD corrections and hadronization effects which, however, turn out to be very small.

The analysis is based on roughly 60,000 accepted hadronic events from the 1992 data and the same number of HVFL Monte Carlo events processed through the same reconstruction program, with standard track and event selection criteria.

The results are shown in Fig. 18. The dotted lines show the Monte Carlo prediction obtained for the primary quarks from the $\mathrm{Z}$ decay without cuts. The upper curve gives the Monte Carlo result for spin-1/2 quarks and the lower one for spin-0 quarks. The experimental distribution 
(full points) is compared to the fully reconstructed Monte Carlo distributions (solid histogram). The prediction for spin-0 quarks was obtained by reweighting the Monte Carlo prediction for spin-1/2 quarks. The sharp drop in the distribution at $\cos \Theta_{\text {THRUST }}=0.8$ is due to the event selection cuts. The spin- $1 / 2$ curve is in excellent agreement with the measurements, while the spin-0 variant is clearly incompatible with the data, confirming that quarks are fermions with spin $1 / 2$.

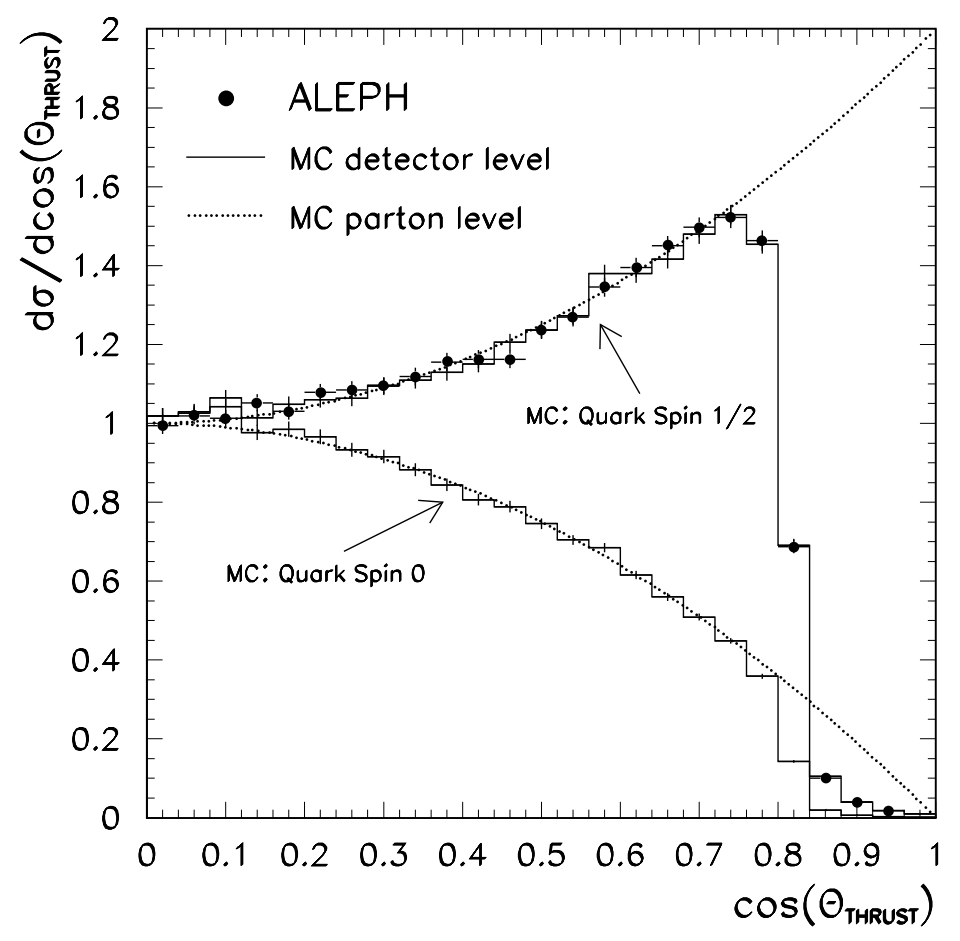

Figure 18: Results from the analysis of the quark spin. The dotted curves show the shape of the theoretical prediction for the primary quarks produced in hadronic $\mathrm{Z}$ decays. The ALEPH raw data (solid points) are compared to the fully simulated Monte Carlo (histograms), for both the spin-1/2 and the spin- 0 hypotheses. The errors are purely statistical. The distributions are normalized to one at $\Theta=90^{\circ}$.

\subsubsection{Gluon Spin}

A study of three-jet events in hadronic Z decays gives insight into the dynamics of perturbative QCD, since at LEP energies the jet structure at the parton level is only slightly changed by fragmentation effects. According to QCD, these events arise from hard non-collinear gluon radiation.

The kinematics of a three-jet event is that of a three-particle decay. For massless jets, of the nine degrees of freedom, after imposing energy and momentum conservation, only five are independent, and of these, three describe the overall orientation of the event. Thus there remain only two independent variables when studying the three-jet topology beyond its angular orientation.

Let $x_{i}$ denote the jet energies normalized to the beam energy,

$$
x_{i}=\frac{2 E_{i}}{\sqrt{s}} \quad i=1,2,3,
$$

with $x_{1}+x_{2}+x_{3}=2$ by energy conservation. In the absence of quark/gluon identification the three jets are energy ordered as $x_{1}>x_{2}>x_{3}$. From these the independent variables used in this analysis are defined as $x_{1}$ and $Z=\frac{1}{\sqrt{3}}\left(x_{2}-x_{3}\right)$, with

$$
\frac{2}{3} \leq x_{1} \leq 1 \quad \text { and } \quad 0 \leq Z \leq \frac{1}{\sqrt{3}}
$$


These two variables can be represented in a triangular Dalitz plot. Figure 19 shows the available phase space for energy-ordered three-jet events and typical jet configurations corresponding to different regions of the phase space.



Figure 19: Phase space for $x_{1}>x_{2}>x_{3}$. The arrow length is proportional to the jet energy.

The three-jet Dalitz plot allows a determination of the gluon spin. The differential cross section for the process $e^{+} e^{-} \rightarrow \mathrm{q} \overline{\mathrm{q}} \mathrm{g}$ has been calculated at leading order both for the vector and the scalar gluon hypothesis. For a vector gluon the leading order matrix element, not requiring any identification of quarks or gluons in the final state, is [15]

$$
\frac{\mathrm{d} \sigma^{\mathrm{v}}}{\mathrm{d} x_{1} \mathrm{~d} x_{2}} \propto\left[\frac{x_{1}^{2}+x_{2}^{2}}{\left(1-x_{1}\right)\left(1-x_{2}\right)}+\operatorname{Permutations}(1,2,3)\right]
$$

This distribution shows two types of singularities, collinear singularities $x_{1} \rightarrow 1$ or $x_{2} \rightarrow 1$ and an infrared singularity $x_{1} \rightarrow 1$ and $x_{2} \rightarrow 1$, i.e. $Z \rightarrow 1 / \sqrt{3}$. In practical applications these singularities are avoided by restricting the phase space to a singularity-free subspace, for example by using the Durham algorithm to define three-particle final states.

The corresponding differential cross section for a scalar gluon is [41]

$$
\frac{\mathrm{d} \sigma^{\mathrm{s}}}{\mathrm{d} x_{1} \mathrm{~d} x_{2}} \propto\left[\frac{x_{3}^{2}}{\left(1-x_{1}\right)\left(1-x_{2}\right)}+\operatorname{Permutations}(1,2,3)-10 \frac{\sum a_{\mathbf{q}}^{2}}{\sum a_{\mathbf{q}}^{2}+v_{\mathbf{q}}^{2}}\right] .
$$

Here $v_{\mathrm{q}}$ and $a_{\mathrm{q}}$ are the vector and axial-vector couplings of a quark to the $\mathrm{Z}$ boson. The sum is taken over all active flavours. The collinear singularities appear again, but as a consequence of the helicity non-conserving coupling of a scalar gluon to the quark current, there is no infrared singularity. This difference in the singularity structure of the matrix elements can be exploited to determine the spin of the gluon by studying the projection of the cross section onto the $Z$ axis.

ALEPH data at $\sqrt{s}=M_{\mathrm{Z}}$ from 1992 have been analyzed. Hadronic events were selected by requiring at least five good charged tracks with a total energy larger than $10 \%$ of the centre-ofmass energy. The analysis then was performed with all charged tracks and neutral objects given 
by the energy flow package, described in 1.2.6. For the final selection the events had to fulfil two further selection criteria: the angle between the beam and the thrust axis had to be larger than $30^{\circ}$ and the total visible energy was required to be larger than $50 \%$ of the centre-of-mass energy.

Three-jet events were selected by the Durham cluster algorithm with the E recombination scheme, with a cutoff parameter $y_{\text {cut }}=0.009$. In total, 193740 events were retained. The jets were then projected onto the event plane and the jet energies reconstructed from the jet directions, using

$$
x_{i}=\frac{2 E_{i}}{\sqrt{s}}=\frac{2 \sin \psi_{i}}{\sum_{j=1}^{3} \sin \psi_{j}} \quad i=1,2,3,
$$

where $\psi_{i}$ is the angle subtended by jets $j$ and $k$, with $\{i, j, k\}$ any permutation of $\{1,2,3\}$. This formula strictly only holds for massless jets. Since the emphasis lies on a comparison between data and theoretical models for massless partons, Eq. (29) was taken as the definition of the experimental observables.

The raw experimental distribution was corrected for detector effects such as geometrical acceptance, detection efficiency and resolution, and effects from initial state radiation using simulated Monte Carlo events from the hadronic event generator HVFL. The size of the corrections was of the order of $5 \%$.

Figure 20 compares the corrected data to the theoretical $Z$ distributions for vector and scalar gluon models. In both cases effects from perturbative higher orders and hadronization effects were estimated from the difference between the leading order analytical formula Eq. (27) or Eq. (28) and the JETSET prediction for the leading order matrix element for massless quarks plus hadronization. As those effects have never been studied for the scalar gluon model, the full size of those higher order effects was taken as the theoretical uncertainty, even though this leads to a clear overestimate of the true uncertainties in the case of the vector gluon.

The impact of the missing perturbative higher orders or mass effects can be estimated by comparing leading order and next-to-leading order matrix elements or massless and massive leading order calculations for the vector gluon. Generally the differences turn out to be negligible.

One finds, even with rather generous assessments of the theoretical uncertainties, that the scalar gluon model is in clear disagreement with the data, and thus can be ruled out as a serious candidate for an alternative to QCD. The vector gluon model provides a very good description of the data.

\subsection{Measurements of the Strong Coupling Constant}

The strong coupling constant, $\alpha_{s}$, is the only free parameter of QCD. Its measurement in different processes serves as a stringent test of the theory, which has to be able to describe a wide range of phenomena with the same value of $\alpha_{s}\left(M_{Z}\right)$, after running to the appropriate energy scale.

The observables which lead to a measurement of $\alpha_{s}$ at LEP can be divided into two broad categories: inclusive and non-inclusive. The first category includes the measurement of the total hadronic decay widths of both the $\mathrm{Z}$ boson and the tau lepton. In the second category belong jet-rate measurements, global event shape variables and scaling violations in fragmentation functions. 




Figure 20: $Z$ distribution. Plotted are the corrected data with full errors and two alternative gluon spin models. For both models, the leading order analytical formula and the Monte Carlo simulation with string fragmentation are shown.

The first observables only depend on total cross sections, with the centre-of-mass energy as the only remaining variable in the process. In this case, non-perturbative effects can be, at worst, of the form $\mathcal{O}\left(\Lambda^{2} / Q^{2}\right)[42]$, with $\Lambda$ below $1 \mathrm{GeV}$, which is very small for $Q^{2}=s=M_{Z}^{2}$. Furthermore, perturbative calculations are easier to carry out, and, as a consequence, more orders in perturbation theory have been computed for the inclusive than for the non-inclusive observables. On the other hand, the dependence of the inclusive observables on $\alpha_{s}$ comes only as a QCD correction to an electroweak cross section. The whole effect, therefore, is small, of order $\mathcal{O}\left(\alpha_{s} / \pi\right)$. Fortunately, high statistics are available at the $\mathrm{Z}$ pole and allow sensitive measurements of $\alpha_{s}$ for inclusive observables.

The non-inclusive observables depend on detailed properties of the final state and, therefore, hadronization effects cannot be ignored. These cannot be computed in perturbative QCD and one has to rely on phenomenological models. In general, these corrections only decrease with energy as $1 / Q[7]$. The high energy available at LEP makes them smaller than at previous $e^{+} e^{-}$ colliders, although they are still non-negligible. These observables have a strong dependence on $\alpha_{s}$ and, typically, are proportional to $\alpha_{s}$. In this case, the statistical sensitivity is not a problem.

In this section, four different methods of measuring the strong coupling constant performed with the ALEPH detector will be presented, and the implications of the $\alpha_{s}$ measurements for the energy dependence of the strong coupling constant will be discussed. 


\subsubsection{Z Hadronic Width}

One of the theoretically best understood determinations of the strong coupling constant comes from the measurement of the hadronic width of the $\mathrm{Z}$ boson or rather, from the ratio of the hadronic and the leptonic widths, $R_{l}=\Gamma_{h} / \Gamma_{l}$. The sensitivity to $\alpha_{s}$ comes from the increase in the $\mathrm{Z}$ partial width to hadron final states due to the effect of both virtual and real gluon radiation. This is an inclusive measurement at very high $Q^{2} \approx 10^{4} \mathrm{GeV}^{2}$. Hence, non-perturbative effects are negligibly small. In addition, it is one of the few observables for which the perturbative prediction is known to $\mathcal{O}\left(\alpha_{s}^{3}\right)[43]$.

The dependence of $R_{l}$ on $\alpha_{s}$ can be parametrized as [44]:

$$
R_{l}=R_{l}^{e w} \cdot\left(1+1.06\left(\frac{\alpha_{s}\left(M_{Z}\right)}{\pi}\right)+0.9\left(\frac{\alpha_{s}\left(M_{Z}\right)}{\pi}\right)^{2}-15\left(\frac{\alpha_{s}\left(M_{Z}\right)}{\pi}\right)^{3}+\mathcal{O}\left(\frac{\alpha_{s}\left(M_{Z}\right)}{\pi}\right)^{4}\right)
$$

where $R_{l}^{e w}=19.932$ for a top mass of $175 \mathrm{GeV}$ and a Higgs mass of $300 \mathrm{GeV}$. This expression includes $b$ quark mass effects up to $\mathcal{O}\left(\alpha_{s}^{2}\right)$, top quark mass effects to $\mathcal{O}\left(\alpha_{s} G_{\mu} m_{t}^{2}\right)$ and QED corrections to $\mathcal{O}\left(\alpha \alpha_{s}\right)$. The overall QCD correction is only of the order of $4 \%$. Therefore, a useful determination of $\alpha_{s}$ requires a very precise measurement of $R_{l}$.

It should be noted that the top quark and Higgs mass dependence of $R_{l}$ is only moderate (see [44], for example). This is so because the loop corrections to the $\mathrm{Z}$ propagator diagram cancel in the ratio $\Gamma_{h} / \Gamma_{l}$. The only residual dependence comes from vertex corrections and photon-Z mixing. For the same reasons, new physics which would mainly manifest itself through propagator corrections would contribute little to $R_{l}$. However, a deviation of the $\mathrm{Z}$ branching ratios from the Minimal Standard Model predictions would affect the determination of $\alpha_{s}$ with this method.

To obtain $R_{l}$ one needs to select hadronic and leptonic events. The methods are described in detail in [45]. The hadronic event selection is similar to that presented in Section 1.3. The leptonic event selection exploits the low multiplicity of the event as well as the particular characteristics of Bhabha, $\mu$-pair and $\tau$-pair final states. The resulting cross sections for data taken in 1989-1995 are given in [46] for the energies around and at the $Z$ peak.

The method for extracting $R_{l}$ consists of fitting the measured cross sections as a function of centre-of-mass energy, $\sqrt{s}$, to the theoretical prediction [45]:

$$
\sigma_{f \bar{f}}(s)=\int_{4 m_{f}^{2}}^{s} d s^{\prime} H\left(s, s^{\prime}\right) \hat{\sigma}_{f \bar{f}}\left(s^{\prime}\right)
$$

where $H\left(s, s^{\prime}\right)$ is the so-called radiator function which takes care of initial state radiation corrections. The reduced cross section $\hat{\sigma}$ is written as

$$
\hat{\sigma}_{f \bar{f}}(s)=\sigma_{f \bar{f}}^{0} \cdot \frac{s \Gamma_{Z}^{2}}{\left(s-M_{Z}^{2}\right)^{2}+\left(\frac{s \Gamma_{Z}}{M_{Z}}\right)^{2}}+(\gamma-Z)+|\gamma|^{2}
$$

where the two last terms represent the interference between the photon- and Z-mediated amplitudes and the photon contribution, respectively, and are taken from theory. This parametrization assumes the validity of QED for the photon exchange part and also takes from the Minimal Standard Model the interference between the photon- and Z-mediated amplitudes. This interference is very small around the $Z$ resonance. In the case of Bhabha scattering, $f=e$, one has to add the $t$-channel photon- and Z-exchange diagrams, also taken from theory. The 
cross section at the peak then can be written in terms of the $\mathrm{Z}$ mass and width and the $\mathrm{Z}$ partial widths to the initial state $\Gamma_{e}$ and the final state $\Gamma_{f}$ :

$$
\sigma_{f \bar{f}}^{0}=\frac{12 \pi}{M_{Z}^{2}} \cdot \frac{\Gamma_{e} \Gamma_{f}}{\Gamma_{Z}^{2}} .
$$

Assuming lepton universality, four parameters are needed to describe the $s$-dependence of the hadronic and leptonic cross sections: the $\mathrm{Z}$ mass $\left(M_{Z}\right)$ and total width $\left(\Gamma_{Z}\right)$, the ratio of hadronic to leptonic partial widths $\left(R_{l}=\Gamma_{h} / \Gamma_{l}\right)$ and the hadronic peak cross section $\left(\sigma_{h}^{0}\right)$.

The computer program MIZA [47], which implements the scheme sketched above, is used to do the fit. The result for $R_{l}$ is

$$
R_{l}=20.766 \pm 0.049
$$

where the error includes all statistical and systematic errors added in quadrature. The statistical error in the lepton sample dominates, where the main systematic errors are related to the knowledge of lepton efficiencies and backgrounds [45]. From Eq. (30) the value obtained for $\alpha_{s}$ is

$$
\alpha_{s}\left(M_{Z}\right)=0.123 \pm 0.007 \pm 0.002_{M_{H}}
$$

where the second uncertainty comes from varying the Higgs mass from 60 to $1000 \mathrm{GeV}$. The top mass has been taken as $175 \pm 6 \mathrm{GeV}$. Combining the results with those of the other three LEP experiments [46], one obtains

$$
\alpha_{s}\left(M_{Z}\right)=0.124 \pm 0.004 \pm 0.002_{M_{H}}
$$

$R_{l}$ is not the only observable at the $\mathrm{Z}$ pole that depends on $\alpha_{s}$; both the total hadronic cross section, $\sigma_{h}^{0}$, and the total $Z$ width, $\Gamma_{Z}$, also have an $\alpha_{s}$ dependence. It is thus possible to derive $\alpha_{s}$ from an overall fit to all electroweak data measured at LEP and elsewhere [46]. The result is similar to that shown above,

$$
\alpha_{s}\left(M_{Z}\right)=0.1202 \pm 0.0033
$$

where the error includes the uncertainty on the Higgs boson mass. In this case the result would also be sensitive to new physics affecting the $\mathrm{Z}$ propagator.

\subsubsection{The Hadronic Width of the Tau}

In a previous paper [48], tau decays have been analyzed to extract $\alpha_{s}$ and non-perturbative parameters of the strong interaction. A similar analysis, based on a larger data set and using improved experimental techniques, is described here. It has been shown [49] that a precise measurement of the strong coupling constant can be achieved using tau decays, through the ratio $R_{\tau}=\Gamma\left(\tau \rightarrow \nu_{\tau}\right.$ hadrons $) / \Gamma\left(\tau \rightarrow \nu_{\tau} l \nu_{l}\right)$, which is defined in the limit of $l$ being a massless lepton. Used in conjunction with $R_{\tau}$, the invariant mass-squared distribution of hadronic tau decay products (hereafter called "the $s$-distribution") provides a handle on some aspects of non-perturbative QCD. 


\section{Theoretical Predictions}

Predictions have been made for $R_{\tau}$ and moments of the $s$-distribution, including perturbative and non-perturbative contributions [49, 50]. Confronting these QCD predictions with data permits the extraction of $\alpha_{s}$ and non-perturbative terms. The moments considered are $D^{k l}=R^{k l} / R_{\tau}=\left\langle\left(1-\frac{s}{m_{\tau}^{2}}\right)^{k}\left(\frac{s}{m_{\tau}^{2}}\right)^{l}\right\rangle$. The prediction for $R_{\tau} \equiv R^{00}$ and the moments reads

$$
R^{k l}=R_{\text {parton }}^{k l} S_{E W}|V|^{2}\left\{1+\delta_{\text {pert }}\left(\alpha_{s}\right)+\delta_{\text {mass }}\left(m_{q}\right)+\delta_{N P}\right\}
$$

where $S_{E W}$ is an electroweak correction factor and $|V|^{2}$ the relevant CKM matrix element(s) squared. The perturbative correction $\delta_{\text {pert }}$ is known to the three loop level. The mass correction term $\delta_{\text {mass }}$ accounts for the non-zero strange quark mass. The non-perturbative contributions are estimated in the framework of the so-called SVZ approach [42]. The prediction

takes the form of a $1 / m_{\tau}^{2}$ expansion: $\delta_{N P} \propto \sum_{D=4,6 \ldots} C_{D} O(D) /\left(-m_{\tau}^{2}\right)^{D / 2}$. Non-perturbative contributions at order $D=2$ are expected to vanish in this approach; the first non-perturbative contribution appears in the $D=4$ terms.

In the following, no $\pi / K$ separation is attempted. The expected $s$-distribution coming from the kaon decay modes is subtracted from the experimental $s$-distribution. The kaon modes are not taken into account in the theoretical predictions, except for $R_{\tau}$, which is extracted from inclusive observables.

\section{Measurement of $R_{\tau}$}

The $R_{\tau}$ ratio is derived from the measurement of the tau leptonic width, obtained from the electron and muon branching ratios, $B_{e}$ and $B_{\mu}$, and from the tau lifetime, $\tau_{\tau}$, assuming $e-\mu-\tau$ universality. The data up to 1993 were analyzed for the leptonic branching ratios [51]. The electronic and muonic branching ratios are $B_{e}=(17.79 \pm 0.13) \%$ and $B_{\mu}=(17.31 \pm 0.12) \%$. The measurement of the tau lifetime is described in [52] and results in $\tau_{\tau}=293.7 \pm 3.1 \mathrm{fs}$, using data collected from 1990 to 1992 . From these three measurements three determinations of the tau branching ratio into a massless lepton and neutrinos, $B_{l}$, can be obtained. They can be combined to give $B_{l}=0.1783 \pm 0.0008$, yielding

$$
R_{\tau}=3.636 \pm 0.025
$$

\section{Invariant Mass-Squared Spectrum of Hadronic Tau Decays}

To reconstruct the $s$-distribution of hadronic tau decays, a selection of $\tau^{+} \tau^{-}$events with an efficiency of $(78.1 \pm 0.1) \%$ is first performed [51]. The overall non-tau background contribution in the hadronic modes, obtained from Monte Carlo simulation corrected with data, amounts to $(0.6 \pm 0.2) \%$. Subsequently, charged particles are identified as electrons, muons or hadrons using a Maximum Likelihood procedure described in [51]. Photons and $\pi^{0}$ s are reconstructed and the invariant mass of the hadronic final state is computed from the charged particles and the $\pi^{0} \mathrm{~s}$. Finally, the detector effects are unfolded from the reconstructed spectrum and the moments are calculated.

The moments that have been chosen are $D^{1 l}, l=0,1,2,3$. The choice of $k=1$ is made in order to suppress the weight in the analysis of the region close to the end point of the spectrum, $s \simeq m_{\tau}^{2}$, which has large experimental errors. 
The reconstruction of photons and $\pi^{0} \mathrm{~s}$ and the subsequent classification of tau decays are described in [53]. Three types of $\pi^{0} \mathrm{~s}$ are reconstructed: i) $\pi^{0} \mathrm{~s}$ from two reconstructed photons; ii) $\pi^{0}$ s from merged photons using a cluster moment analysis; iii) single photons taken as $\pi^{0}$ s. The tau decays are classified into one of the twelve classes $\tau \rightarrow \nu_{\tau} X$, $X=e \nu_{e}, \mu \nu_{\mu}, m h^{ \pm} n \pi^{0}(m=1,3,5)$ where $h$ stands for $\pi$ or $K$. For the one- and three-prong modes, tau decays with $n=0,1,2$ and more than two are classified separately. In five-prong tau decays, only decays with or without $\pi^{0}$ s are differentiated.

A class is subdivided into three subclasses, according to the $\pi^{0}$ reconstruction type described above. This makes it possible to take into account the differences in purity and accuracy of the mass reconstruction between these subclasses. Overall, there are 26 subclasses. After the classification, the $s$-distributions are built in each class and the (small) non-tau background, as well as the contributions from kaon modes, are subtracted, using the Monte Carlo simulation.

In order to extract the true $s$-distribution of taus decaying into hadronic channels from the reconstructed spectrum, an unfolding procedure is needed. For that purpose, the unfolding method developed for the previous analysis [48] has been improved. This improvement refers essentially to the treatment of tau background events in a given tau decay channel. Previously, these events were simply subtracted; they are now included in the unfolding procedure using non-diagonal probability matrices predicted by Monte Carlo simulation [54].

The unfolded $s$-distribution from 1992 ALEPH data, corresponding to approximately $5 \times 10^{4}$ reconstructed tau decays, is shown in Fig. 21, where only statistical errors are shown. It should be noted that statistical as well as systematic errors are strongly correlated between different bins. The moments are obtained from the distribution of Fig. 21 and are given in Table 11.

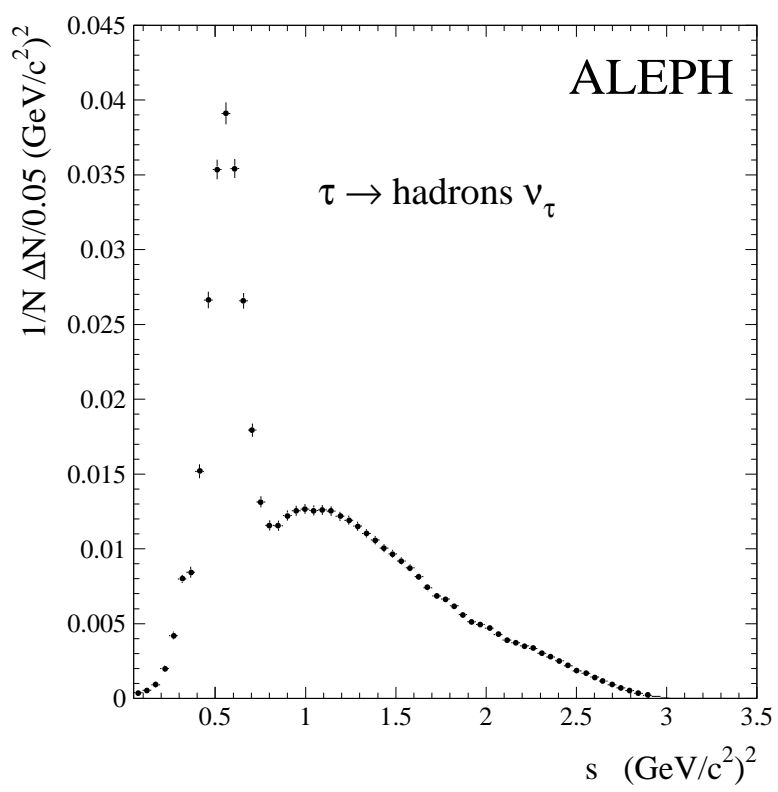

Figure 21: Unfolded $s$-distribution with statistical errors only. The contribution of the $\tau \rightarrow \pi \nu$ final state is not shown.

The systematic uncertainties include contributions from the limited Monte Carlo statistics, the electromagnetic calorimeter energy calibration where a global uncertainty of $(0.3+3 / \sqrt{E}) \%$, with $E$ in $\mathrm{GeV}$, and a pedestal error of $35 \mathrm{MeV}$ is assumed, the photon reconstruction procedure (the energy threshold fixed at $300 \mathrm{MeV}$ has been varied by $\pm 50 \mathrm{MeV}$ to cover 
eventual inefficiencies in the low-energy photon reconstruction, an excess of fake photons of roughly $20 \%$ found in the data at low energy was simulated in the Monte Carlo to estimate the corresponding systematic uncertainty), the $\pi^{0}$ reconstruction, the charged particle identification, the reconstruction of the three-prong modes where the reconstruction efficiency of highly collimated tracks was studied in addition to effects coming from secondary nuclear interactions, the non-tau background subtraction and finally the unfolding procedure. The dominant systematic errors are those coming from the understanding of the electromagnetic calorimeter.

\begin{tabular}{|c||c|c|c|c|}
\hline$l$ & 0 & 1 & 2 & 3 \\
\hline$D_{\tau}^{1 l}$ & 0.7228 & 0.1569 & 0.0570 & 0.0256 \\
\hline$\sigma[$ stat $]$ & 0.0019 & 0.0007 & 0.0005 & 0.0003 \\
\hline$\sigma[$ exp $]$ & 0.0060 & 0.0020 & 0.0013 & 0.0008 \\
\hline$\sigma[$ theo $]$ & 0.0034 & 0.0029 & 0.0004 & 0.0002 \\
\hline
\end{tabular}

Table 11: The measured moments of the hadronic invariant mass-squared distribution, together with their statistical, total experimental and theoretical errors.

\section{Fit Results}

The ratio $R_{\tau}$ and the four $D^{1 l}$ moment measurements are combined with their experimental and theoretical covariance matrices in a fit for $\alpha_{s}$ and the three main non-perturbative terms. The results are given in Table 12. The first column shows the global fit result, the second gives the result of a fit for $\alpha_{s}$ using $R_{\tau}$ only, using as input for the non-perturbative terms the estimates quoted in [49] and the last column, a fit for $\alpha_{s}$ using the moments only. The two experimentally uncorrelated determinations of $\alpha_{s}$ using $R_{\tau}$ or the moments are in good agreement.

\begin{tabular}{|c||c|c|c|}
\hline & $R_{\tau}+D^{1 l}$ & $R_{\tau}$ & $D^{1 l}$ \\
\hline \hline$\alpha_{s}$ & $0.353 \pm 0.022$ & $0.366 \pm 0.024$ & $0.365 \pm 0.055$ \\
\hline$\left\langle\frac{\alpha_{s}}{\pi} g g\right\rangle$ & $+0.004 \pm 0.012$ & $0.02 \pm 0.02$ & $0.02 \pm 0.02$ \\
\hline$O(6)$ & $-0.001 \pm 0.002$ & $0.002 \pm 0.002$ & $0.002 \pm 0.002$ \\
\hline$O(8))$ & $+0.002 \pm 0.002$ & $0.0 \pm 0.007$ & $0.0 \pm 0.007$ \\
\hline$\chi^{2} /$ d.o.f. & 0.1 & - & 1.1 \\
\hline
\end{tabular}

Table 12: Fit results for ALEPH 1992 data. The unit for the dimension $D$ term is $\mathrm{GeV}^{D}$. Numbers in italics are input values to the fits.

Taking into account the results of Table 12 and their correlations shown in Table 13, one can extract the total non-perturbative contribution to $R_{\tau}$ :

$$
\begin{aligned}
& \delta R_{\tau}=3 \times \delta_{N P} \\
& \delta_{N P}=(-0.02 \pm 0.5) \%
\end{aligned}
$$

Extrapolating the $\alpha_{s}$ measurement from the tau mass scale to the Z mass scale according to the method of ref. [55], yields

$$
\alpha_{s}\left(M_{\mathrm{Z}}\right)=0.1221 \pm 0.0015_{\text {exp }} \pm 0.0018_{\text {theo }} \pm 0.0010_{\text {extrap }},
$$




\begin{tabular}{|c|c|c|c|}
\hline ALEPH & $\alpha_{s}$ & $\left\langle\frac{\alpha_{s}}{\pi} g g\right\rangle$ & $O(6)$ \\
\hline$\left\langle\frac{\alpha_{s}}{\pi} g g\right\rangle$ & -0.41 & - & - \\
\hline$O(6)$ & +0.41 & -0.91 & - \\
\hline$O(8)$ & -0.39 & +0.92 & -0.98 \\
\hline
\end{tabular}

Table 13: Correlation coefficients between $\alpha_{s}$ and the measured non-perturbative terms.

where the first (second) error accounts for experimental (theoretical) uncertainties and the third error reflects uncertainties in the extrapolation.

The estimates of theoretical uncertainties are performed as in ref. [48]. The largest uncertainty stems from the missing fourth order coefficient of the perturbative expansion. The contribution of the theoretical uncertainties to the overall error is numerically equal to the contributions of experimental uncertainties, where the latter are dominated by the statistical error on the leptonic branching ratios. However, the nature of the theoretical uncertainties and the proper procedure to be used to evaluate them are still unclear. As a result, a range of estimates can be found in the literature: the theoretical precision of the $\alpha_{s}\left(M_{\mathrm{Z}}\right)$ determination from $R_{\tau}$ alone is evaluated as $\sim 0.003$ in [56], 0.005 in [57] and $\sim 0.006$ in [58].

\subsubsection{Event Shapes and Jet Rates}

The topology of hadronic events in $e^{+} e^{-}$collisions is modified by the effects of gluon radiation, giving rise to events which differ from the collimated two-jet topology coming from the fragmentation of pure $q \bar{q}$ events. Since the amount of gluon radiation is directly proportional to the strong coupling constant, studying the topology of hadronic decays of the Z boson will provide a measurement of $\alpha_{s}\left(M_{Z}\right)$.

The strategy consists of finding variables which characterize the "three-jetness" of the event. In order to be able to perform reliable perturbative calculations, the variables have to be infrared and collinear safe, i.e. insensitive to soft and/or collinear gluon emission.

Many variables have been defined which fulfil these properties [59]. One of the most widely used is the thrust, $T$, defined in Section 2. The thrust distribution is shown in Fig. 7. The thrust can take values from 0.5 , corresponding to a totally spherical event, to 1 , corresponding to a perfect two-jet event. Multiple soft gluon emission and fragmentation effects populate the region close to $T=1$, where most of the events lie. This is the so-called two-jet region. Hard gluon emission creates the tail of the distribution towards lower values of $T$. The thrust distribution is in itself already proportional to $\alpha_{s}$ in leading order,

$$
\frac{1}{\sigma} \frac{d \sigma}{d T} \propto \alpha_{s} A(T)+\cdots,
$$

which implies that the distribution is statistically very sensitive to $\alpha_{s}$. This can be extended to the other event shape variables that are analyzed. They, in general, will be called $y$, with $y=0$ corresponding to the two-jet limit, i.e., for thrust, $y=1-T$.

The data analysis follows exactly that explained in Section 1.3, and is based only on charged particles. The experimental systematic errors, as defined in Section 2, are larger than the statistical errors but considerably smaller than the theoretical uncertainties. 


\section{Analysis Using Second Order QCD Predictions}

The QCD predictions for all the event shape variable distributions are known to second order in $\alpha_{s}[16]$, i.e.

$$
\frac{1}{\sigma} \frac{d \sigma}{d y}=\frac{\alpha_{s}(\mu)}{2 \pi} \cdot A(y)+\left(\frac{\alpha_{s}(\mu)}{2 \pi}\right)^{2} \cdot\left[B(y)+2 \pi b_{0} \ln \left(\frac{\mu^{2}}{s}\right) \cdot A(y)\right] .
$$

The functions $A(y)$ and $B(y)$ are specific for every event shape variable and contain the full information of the second order matrix elements. The parameter $\mu$ denotes the arbitrary renormalization scale used for the calculation. A complete all-order calculation would not depend on the value of $\mu$ chosen. However, a truncated second order prediction does, and this will create a large uncertainty in the final result.

The prediction corresponds to a partonic final state with, at most, four partons. To obtain a prediction for multi-hadronic final states, the above expression is convoluted with the predictions obtained from phenomenological hadronization models. By using the Lund second order matrix element model [60] tuned to the data, not only hadronization corrections but also perturbative higher order effects are effectively included, although for a fixed value of $\alpha_{s}$.

The ratio of the predictions of this model at hadron and parton level are shown in Fig. 22 for two different variables: the differential two-jet rate using the JADE scheme, $y_{3}$, and the oblateness, $O$. While differences between hadron and parton level are minor for the former (mean value close to zero and small width), they are much bigger for the latter. Therefore, $y_{3}$ should be considered a more reliable variable for the measurement of $\alpha_{s}$ than $O$.

For each event shape variable considered, the measured distribution corrected for detector effects is fitted to the convolution of the second order prediction with the higher order and hadronization corrections resulting from the phenomenological models. By using a number of them differing either in higher orders (models based on the exact second order QCD [60] and models implementing leading-logarithm parton showers [61]) or in the hadronization phase (models based on string fragmentation [61] and models based on cluster fragmentation [62]), systematic errors due to hadronization and higher order uncertainties are obtained. Details can be found in ref. [63].

The results are shown in Table 14 for the event shape variables analyzed in [63]. The renormalization scale has been fixed to $\mu=M_{Z} / 2$, that is, half-way between the centre-of-mass energy and the non-perturbative scale. It can be seen that the best measurement is by far that provided by the differential two-jet rate, $y_{3}$. Therefore, this was chosen as the final result of the analysis, leading to:

$$
\alpha_{s}\left(M_{Z}\right)=0.121 \pm 0.002(\text { stat }) \pm 0.003(\text { sys }) \pm 0.007(\text { theo })
$$

for $\mu=M_{Z} / 2$. If the renormalization scale is changed to the bottom quark mass or to $M_{Z}$, the result varies by -0.012 and +0.007 , respectively.

\section{Analysis Using Pre-Clustered Event Shape Variables in Second Order QCD}

As has been seen in the previous section, higher order and hadronization effects prevent the effective use of most of the existing event shape variables to determine $\alpha_{s}$ accurately. An idea to improve the situation was presented in [64], where the event shape variables are not computed 

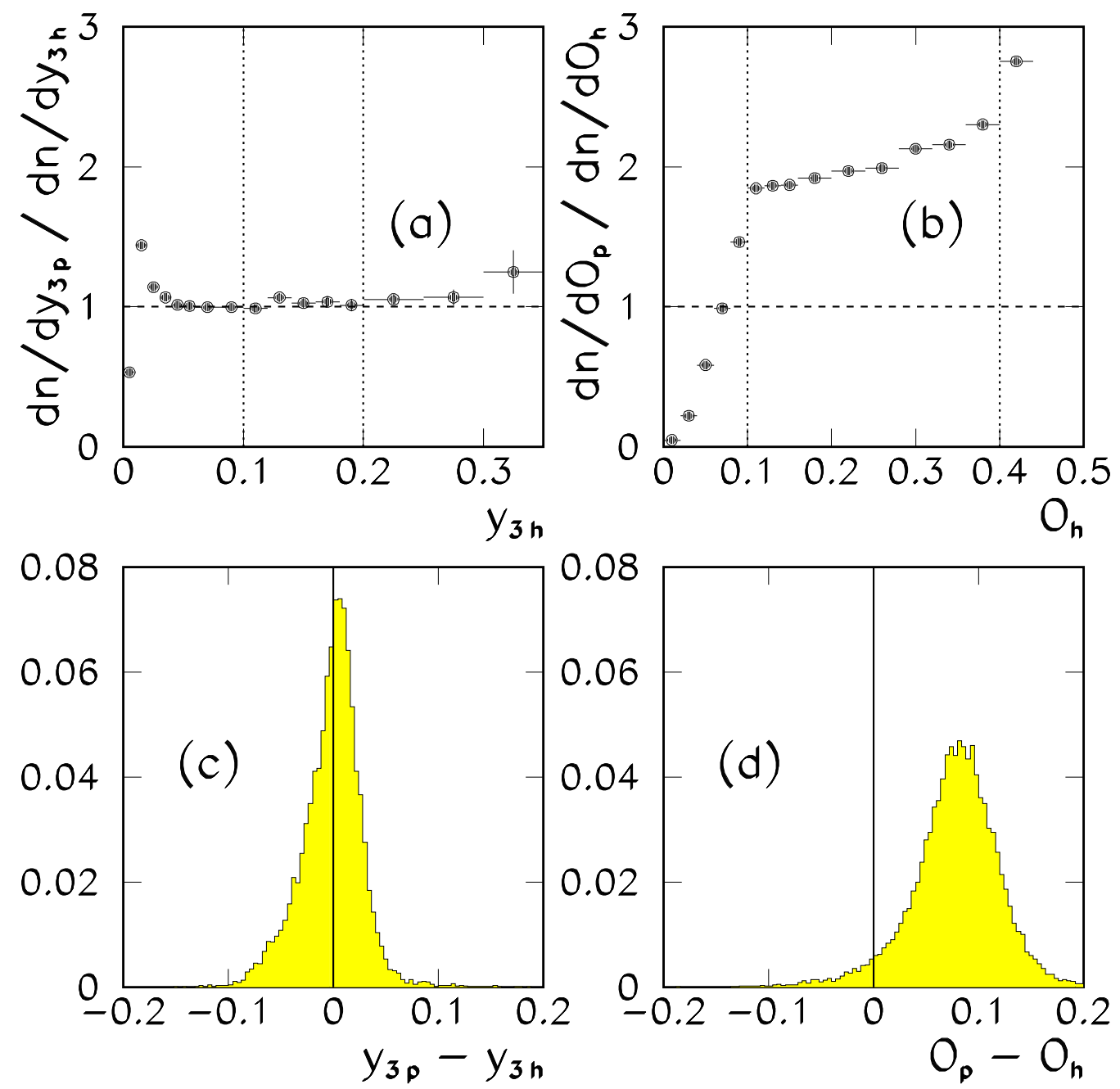

Figure 22: The ratio (upper plots) and the event-by-event difference (lower plots) between the predictions at parton and hadron level for the differential two-jet rate using the JADE scheme, $y_{3}$, and the oblateness, $O$.

\begin{tabular}{|l|c|}
\hline Distribution & $\alpha_{s}\left(M_{Z}\right)$ \\
\hline Thrust & $0.119 \pm 0.004 \pm 0.013$ \\
Oblateness & $0.186 \pm 0.003 \pm 0.036$ \\
$\mathrm{C}$ & $0.112 \pm 0.004 \pm 0.017$ \\
$M_{H, T}^{2} / s$ & $0.136 \pm 0.004 \pm 0.012$ \\
$M_{D, T}^{2} / s$ & $0.142 \pm 0.004 \pm 0.014$ \\
$y_{3}$ & $0.121 \pm 0.004 \pm 0.007$ \\
\hline
\end{tabular}

Table 14: Results for $\alpha_{s}\left(M_{Z}\right)$ using second order predictions with a fixed renormalization scale $\mu=M_{Z} / 2$. The first error is experimental while the second includes theoretical errors, but it does not include any renormalization-scale uncertainty.

from the single particle momenta of the final state, but from clusters of neighbouring particles in phase space. Naively, these clusters should more closely resemble the structure of a purely 
partonic final state as accessible in finite order perturbation theory.

The procedure for the analysis of the data and the definition of the experimental systematic errors are identical to those used in the analysis reviewed in the previous section. Pre-clustering of the final state particles is made using the JADE clustering algorithm with a certain value of the resolution parameter, $y_{c u t}$. The second order QCD prediction is obtained by using the Monte Carlo event generator program EVENT [65] and applying the same clustering algorithm to the generated partonic final state. These predictions depend now on the value of $y_{\text {cut }}$.

Fragmentation and higher order effects are studied in the same way as in ref. [63] and a substantial reduction in the uncertainties is found for appropriate choices of $y_{c u t}$. The values of $\alpha_{s}$ obtained do not depend significantly on $y_{c u t}$ for values above 0.02 . Figure 23 shows the measured energy-energy correlation (EEC) distribution with preclustering together with the ratio of hadron and parton level distributions from two hadronization models for two different values of $y_{\text {cut }}$. The corrections from the models are below $20 \%$ in all cases. The preclustered EEC distribution is defined as

$$
\langle E E C(\cos \chi)\rangle_{\text {bin } k}=\frac{1}{N_{\text {events }}} \sum_{\text {events }} \sum_{i, j=1}^{N_{c l}} \frac{E_{i} E_{j}}{E_{c m}^{2}} \frac{1}{\Delta \cos \chi} \int_{b i n k} \delta\left(\cos \chi_{i j}-\cos \chi^{\prime}\right) d \cos \chi^{\prime},
$$

where $\chi_{i j}$ is the angle between the clusters $i$ and $j$, the sum over $i$ and $j$ is made over all pairs of $N_{c l}$ clusters in an event, and is then averaged over all events.

The results obtained for the event shape variables studied can be seen in Table 15 . The preclustering increases substantially the correlations between the variables. The combined value, taking into account all correlations and choosing $\mu=M_{Z} / 2$, is

$$
\alpha_{s}\left(M_{Z}\right)=0.117 \pm 0.005
$$

where the error includes both experimental and theoretical uncertainties, but it does not include explicitly the uncertainty related to the choice of renormalization scale. The result moves by ${ }_{-0.009}^{+0.006}$ for scales ranging from the $b$ quark mass up to $M_{Z}$. The final result is in agreement with that obtained previously from $y_{3}$.

\begin{tabular}{|l|c|}
\hline Distribution & $\alpha_{s}\left(M_{Z}\right)$ \\
\hline EEC & $0.118 \pm 0.002 \pm 0.005$ \\
$\mathrm{~T}$ & $0.123 \pm 0.004 \pm 0.006$ \\
$\mathrm{C}$ & $0.124 \pm 0.004 \pm 0.006$ \\
$\mathrm{O}$ & $0.115 \pm 0.004 \pm 0.005$ \\
\hline
\end{tabular}

Table 15: Results for $\alpha_{s}\left(M_{Z}\right)$ using preclustered variables and second order predictions with a fixed renormalization scale $\mu=M_{Z} / 2$. The first error is experimental while the second includes theoretical errors, but it does not include any renormalization-scale uncertainty. It should be noted that the four results are strongly correlated, due to the preclustering.

\section{Analysis Using All-Orders Resummed Predictions}

Although QCD predictions to $\mathcal{O}\left(\alpha_{s}^{3}\right)$ are not within immediate reach, there has been in the past few years significant theoretical progress concerning the resummation of large logarithms in the perturbation series to all orders of $\alpha_{s}[66]$. 


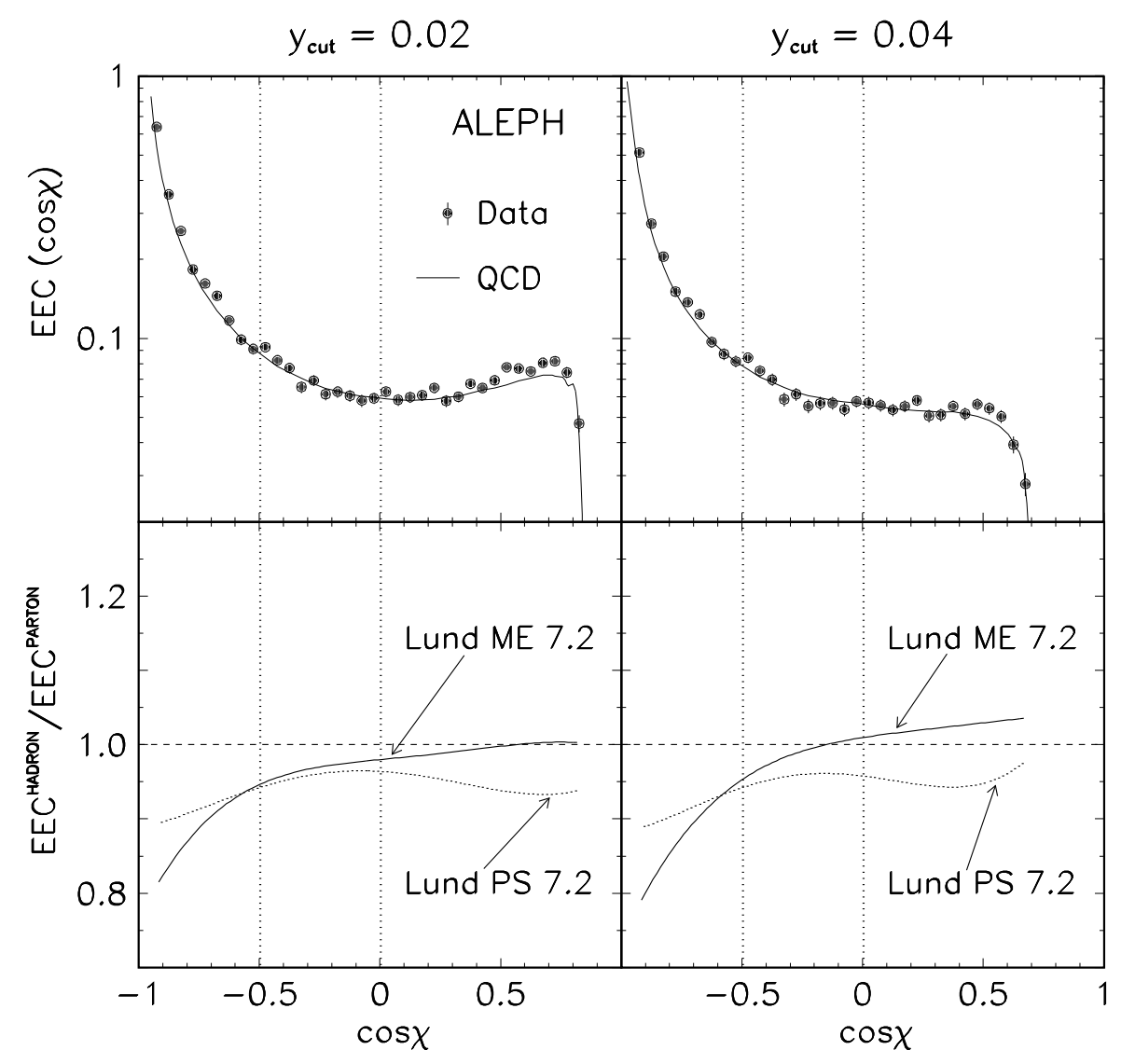

Figure 23: EEC distribution with preclustering together with the ratio of hadron and parton level distributions from two hadronization models for two different values of $y_{c u t}$. The vertical dashed lines indicate the fit range.

These higher order calculations are similar to the parton shower calculations implemented in the Monte Carlo programs which most successfully describe the data. The corrections due to the transition between partons and hadrons and their uncertainty will be smaller. Furthermore, the uncertainties related to the renormalization scale will be reduced since higher orders are partially included. Finally, the range of validity of the predictions will extend further into the two-jet region.

For a general event shape variable $Y$ for which the theoretical prediction can be exponentiated, the resummed prediction for the cumulative distribution, defined by

$$
R\left(y, \alpha_{s}\right)=\frac{1}{\sigma_{\text {tot }}} \sigma(Y<y),
$$

can be written in the following way:

$$
\ln R\left(y, \alpha_{s}\right)=L \cdot f_{L L}\left(\alpha_{s} L\right)+f_{N L L}\left(\alpha_{s} L\right)+\text { subleading terms },
$$

where $L=-\ln y$ becomes large in the two-jet region. The functions $f_{L L}$ and $f_{N L L}$ depend only on the product of $\alpha_{s}$ and $L$. The first two terms in Eq. (46) represent the leading and the next-toleading logarithms. They have been computed for a number of variables, including thrust [67], heavy jet mass [68] and differential two-jet rate with the Durham cluster algorithm [69]. The 
calculation of the next-to-leading logarithm function used in this analysis is not complete for the last variable ${ }^{1}$.

The expansion of Eq. 46 in powers of $\alpha_{s}$ is shown in Table 16. The first column $\left(\propto \alpha_{s}^{n} L^{n+1}\right)$ represents the leading logarithms, the second column $\left(\propto \alpha_{s}^{n} L^{n}\right)$ the next-to-leading logarithms and the first two rows represent the complete $\mathcal{O}\left(\alpha_{s}^{2}\right)$ predictions [16, 59]. An improved prediction

\begin{tabular}{|c||c|c|c|c|c|c|}
\hline & Leading Log & Next-to-Leading Log & \multicolumn{4}{|c|}{ Subleading } \\
\hline \hline First Order & $\alpha_{s} L^{2}$ & $\alpha_{s} L$ & $\alpha_{s}$ & $\ldots$ & \multicolumn{2}{|c|}{} \\
\hline Second Order & $\alpha_{s}^{2} L^{3}$ & $\alpha_{s}^{2} L^{2}$ & $\alpha_{s}^{2} L$ & $\alpha_{s}^{2}$ & $\ldots$ & \\
\hline Third Order & $\alpha_{s}^{3} L^{4}$ & $\alpha_{s}^{3} L^{3}$ & $\alpha_{s}^{3} L^{2}$ & $\alpha_{s}^{3} L$ & $\alpha_{s}^{3}$ & $\ldots$ \\
\hline$\vdots$ & $\vdots$ & $\vdots$ & & & & \\
\hline
\end{tabular}

Table 16: Schematic representation of the order by order expansion of theoretical prediction in leading logarithms, next-to-leading logarithms and subleading logarithms.

can thus be constructed by combining the exact second order predictions with the leading and next-to-leading logarithms of Eq. (46), starting in $\mathcal{O}\left(\alpha_{s}^{3}\right)$. There are several ways this matching can be done [71], either for $\ln R$ ("In $R$ matching") or for $R$ (" $R$ matching"). They differ in exactly which terms are being exponentiated. Differences start always at $\mathcal{O}\left(\alpha_{s}^{3}\right)$.

In ref. [71] the variables thrust, heavy jet mass and differential two-jet rate with the Durham cluster algorithm have been used to measure $\alpha_{s}$ within the framework of the resummed predictions. Raw data distributions are corrected for detector effects following the scheme outlined in Section 1.3.

The parton-level predictions have been folded with hadronization effects obtained from parton shower models JETSET 7.2 and HERWIG 5.3 to obtain predictions at the hadron level, which are compared with the data. The data and the QCD fit are shown in Fig. 24.

The final result for each distribution is

$$
\begin{aligned}
& \left.\alpha_{s}\left(M_{Z}\right)\right|_{y_{3}}=0.1257 \pm 0.0010_{\text {stat }} \pm 0.0025_{\text {syst }} \pm 0.0007_{\text {hadr }} \pm 0.0043_{\text {theo }} \\
& \left.\alpha_{s}\left(M_{Z}\right)\right|_{T}=0.1263 \pm 0.0008_{\text {stat }} \pm 0.0010_{\text {syst }} \pm 0.0028_{\text {hadr }} \pm 0.0065_{\text {theo }} \\
& \left.\alpha_{s}\left(M_{Z}\right)\right|_{\rho}=0.1243 \pm 0.0010_{\text {stat }} \pm 0.0033_{\text {syst }} \pm 0.0042_{\text {hadr }} \pm 0.0057_{\text {theo }}
\end{aligned}
$$

where the first error is statistical, the second estimates the experimental systematics, the third concerns the hadronization correction, and the last is the estimate of theoretical uncertainties. The hadronization uncertainty takes into account, among other things, the differences in the results obtained when folding the parton distributions with different hadronization models. The theoretical uncertainty covers the uncertainty due to the choice of matching scheme as well as the variation of the renormalization scale used in the calculation in the range $-1 \leq \ln \mu^{2} / s \leq+1$. The central values are given at $\mu^{2}=s$. In contrast with most analyses using second order QCD, this analysis using resummed predictions does not prefer values of $\mu$ much smaller than the centre-of-mass energy.

Combining the three previous results (47), taking into account all correlations, leads to the final result for the strong coupling constant

$$
\alpha_{s}\left(M_{Z}\right)=0.1251 \pm 0.0009_{\text {stat }} \pm 0.0021_{\text {syst }} \pm 0.0007_{\text {hadr }} \pm 0.0038_{\text {theo }}=0.125 \pm 0.005 .
$$

\footnotetext{
${ }^{1}$ Recently, a first work on the complete resummation of the next-to-leading terms has appeared [70].
} 



Figure 24: Experimental distributions (statistical errors only) together with bands covering the predictions using the three hadronization models and the central values of $\alpha_{s}$ for $\mu=M_{Z}$ and $R$ matching. The curves are the predictions for the same values of $\alpha_{s}$ without hadronization corrections.

This result is compatible with the results obtained using second order predictions with and without preclustering. However, the error is considerably smaller, essentially because of the reduced theoretical error.

\subsubsection{Scaling Violations in Fragmentation Functions}

The study of scaling violations in structure functions in deep-inelastic lepton-nucleon scattering played a fundamental role in establishing QCD as the theory of strong interactions. QCD predicts similar scaling violations in the fragmentation functions of quarks and gluons. In an electron-positron collider this translates into the fact that the distributions of the scaled-energy $x \equiv 2 \mathrm{E} / \sqrt{s}$ of final state particles in hadronic events depend on the centre-of-mass energy $\sqrt{s}$. A measurement of the scaled-energy distributions at different centre-of-mass energies compared to the QCD prediction allows a determination of the only free parameter of QCD, $\alpha_{s}$. An analysis of this type was presented in [72]. 
The general form for the inclusive distribution of $x$ and polar angle $\theta$ with respect to the beam axis is given by [73]:

$$
\frac{d^{2} \sigma(s)}{d x d \cos \theta}=\frac{3}{8}\left(1+\cos ^{2} \theta\right) \frac{d \sigma^{T}(s)}{d x}+\frac{3}{4} \sin ^{2} \theta \frac{d \sigma^{L}(s)}{d x}+\frac{3}{4} \cos \theta \frac{d \sigma^{A}(s)}{d x},
$$

where $T, L$ and $A$ refer to the transverse, longitudinal and asymmetric cross sections. Integrating over $\cos \theta$ one obtains the inclusive cross section

$$
\frac{d \sigma(s)}{d x}=\frac{d \sigma^{T}(s)}{d x}+\frac{d \sigma^{L}(s)}{d x}
$$

which carries most of the weight in the analysis. The total cross section is dominated by the transverse component. The longitudinal component arises from QCD corrections and is only used to constrain the gluon fragmentation function.

The cross sections are related to fragmentation functions $D_{i}, i=u, d, s, c, b$, for quarks and $D_{g}$ for gluons, which describe the momentum spectrum of final state particles from a single parton, by a convolution with coefficient functions $C_{q}, C_{g}$, computed in perturbative QCD [74]:

$$
\begin{aligned}
\frac{d \sigma(s)}{d x} & =2 \sigma_{0}(s) \int_{x}^{1} \frac{d z}{z} C_{q}\left(z, \alpha_{s}\left(\mu_{F}\right), \mu_{F}^{2} / s\right) \sum_{i=u, d, s, c, b} w_{i}(s) D_{i}\left(x / z, \mu_{F}^{2}\right) \\
& +2 \sigma_{0}(s) \int_{x}^{1} \frac{d z}{z} C_{g}\left(z, \alpha_{s}\left(\mu_{F}\right), \mu_{F}^{2} / s\right) D_{g}\left(x / z, \mu_{F}^{2}\right)
\end{aligned}
$$

Here $\sigma_{0}(s)$ is the Born cross section at the centre-of-mass energy $\sqrt{s}$ and $w_{i}$ is the relative electroweak cross section for the production of primary quarks of type $i$. The scale $\mu_{F}$ is an arbitrary factorization scale where the fragmentation functions are evaluated. The fragmentation functions themselves cannot be calculated within perturbative QCD, but once they are fixed at some parametrization scale $\sqrt{s_{0}}$, their energy evolution is predicted.

The QCD scaling violations are described by the DGLAP evolution equations [75]

$$
\frac{d D_{j}(x, s)}{d \ln s}=\sum_{i=u, d, s, c, b, g} \int_{x}^{1} \frac{d z}{z} P_{i j}\left(z, \alpha_{s}\left(\mu_{R}\right), \mu_{R}^{2} / s\right) D_{i}(x / z, s),
$$

where $\mu_{R}$ is the renormalization scale and $P_{i j}$ are the splitting kernels [76]. Both the coefficient functions and the splitting kernels in the $\overline{\mathrm{MS}}$ scheme can be found, for example, in ref. [73]. For the analysis presented here the scales $\mu_{R}$ and $\mu_{F}$ are varied around the natural scale $\sqrt{s}$ as in ref. [71].

The formalism developed above describes only the perturbative component of the scaling violations. Corrections due to resonance decays that scale like $\mathrm{m}^{2} / \mathrm{s}$, quark-mass effects and non-perturbative effects are discussed in detail in ref. [73]. The latter manifest themselves as power-law corrections of $\mathcal{O}\left(1 / \sqrt{s^{k}}\right)$ to the logarithmic scaling violations expected from perturbative QCD. Phenomenological arguments [73] suggest $k=1$. A simple way of incorporating non-perturbative effects is by changing variables and relating the perturbative variable $x$ to the measured quantity $x^{\prime}$ through a function $x=g\left(x^{\prime}\right)$. The ansatz

$$
x=x^{\prime}+h_{0}\left(\frac{1}{\sqrt{s}}-\frac{1}{\sqrt{s_{0}}}\right)
$$

with one effective parameter $h_{0}$, supported by Monte Carlo studies, is used to parametrize all power-law corrections over the energy range between $22 \mathrm{GeV}$ and $91.2 \mathrm{GeV}$ covered by the data analyzed here. 
Because the fragmentation functions depend on the quark mass, and the relative cross section for each flavour depends on $\sqrt{s}$, a measurement of $\alpha_{s}$ from scaling violations in inclusive momentum distributions requires knowledge of the fragmentation functions for all quark flavours at one energy. Information about the various quark flavours is extracted from the data, by controlling the flavour composition of the data sample using appropriate tagging techniques. The impact parameter tag described in Section 1.2 is used to select a $b$ quark enriched sample and a light $(u, d, s)$ quark enriched sample. The same technique combined with a tag based on shape variables [78] is applied to obtain a $c$ quark enriched sample. The scaled energy distribution is measured in an inclusive sample and in the three tagged samples.

Equations (49) and (50) show that the gluon fragmentation function is also needed. A direct measurement of the gluon fragmentation function is obtained from three-jet events where jets from well separated gluons are tagged by default when the other two jets contain long-lived particles (Section 4.5). The gluon fragmentation function is also extracted by measuring the longitudinal and transverse cross sections, which are related to the gluon fragmentation function according to $[73]$

$$
\frac{1}{\sigma_{\text {tot }}} \frac{d \sigma^{L}}{d x}=\frac{\alpha_{s}}{2 \pi} \int_{x}^{1} \frac{d z}{z}\left[\frac{1}{\sigma_{t o t}} \frac{d \sigma^{T}}{d z}+4\left(\frac{z}{x}-1\right) D_{g}(z)\right]+\mathcal{O}\left(\alpha_{s}^{2}\right) .
$$

Truncating the above expression at $\mathcal{O}\left(\alpha_{s}\right)$, the parameter $\alpha_{s}$ becomes an effective leading-order coupling constant which must not be confused with the next-to-leading order running coupling constant appearing in Eqs. (49),(50). Because of this, it will be referred to as $\beta_{s}$ in the following.

The longitudinal and transverse cross sections are measured by weighting the doubledifferential cross section with respect to $x$ and $\cos \theta$ with the appropriate weight to project onto the $\left(1+\cos ^{2} \theta\right)$ component (transverse) or the $\sin ^{2} \theta$ component (longitudinal).

Systematic errors in all distributions, due to imperfect detector simulation or biases due to the hadron production model used to correct the data, are estimated using the techniques described in Section 2. On top of those, additional systematic errors are assigned to the longitudinal and transverse distributions (especially sensitive to tracking inefficiencies at low angles) [72] and a $1 \%$ normalization error is attributed to all distributions, according to the findings of ref. [79]. Systematic uncertainties specific to the flavour tagging procedures are treated separately in the $\alpha_{s}$ determination. Figure 25 shows the measured distributions. One clearly sees the difference between light and heavy flavour enriched samples. The errors include all bin-to-bin errors (statistical and systematic) added in quadrature as well as an overall $1 \%$ normalization error. Systematic errors dominate everywhere. The transverse distribution is almost identical to the unweighted distribution for all flavours and is not shown.

In addition to the ALEPH data, inclusive charged particle spectra from TASSO [80] at $\sqrt{s}=22,35$ and $45 \mathrm{GeV}$, MARK II [81] and TPC/2 $\gamma$ [82] at $\sqrt{s}=29 \mathrm{GeV}$, CELLO [83] at $\sqrt{s}=35 \mathrm{GeV}$, AMY [84] at $\sqrt{s}=55 \mathrm{GeV}$ and DELPHI [85] at $\sqrt{s}=91.2 \mathrm{GeV}$ have been used in the analysis. Lower-energy data were not used because of the possible larger size of the power-law corrections.

The fragmentation functions for the different flavours and for the gluon are parametrized using the functional form

$$
x D_{i}\left(x, s_{0}\right)=N_{i} \frac{(1-x)^{a_{i}} x^{b_{i}} \exp \left(-c \ln ^{2} x\right)}{\int_{0.1}^{0.8} d x(1-x)^{a_{i}} x^{b_{i}} \exp \left(-c \ln ^{2} x\right)}
$$




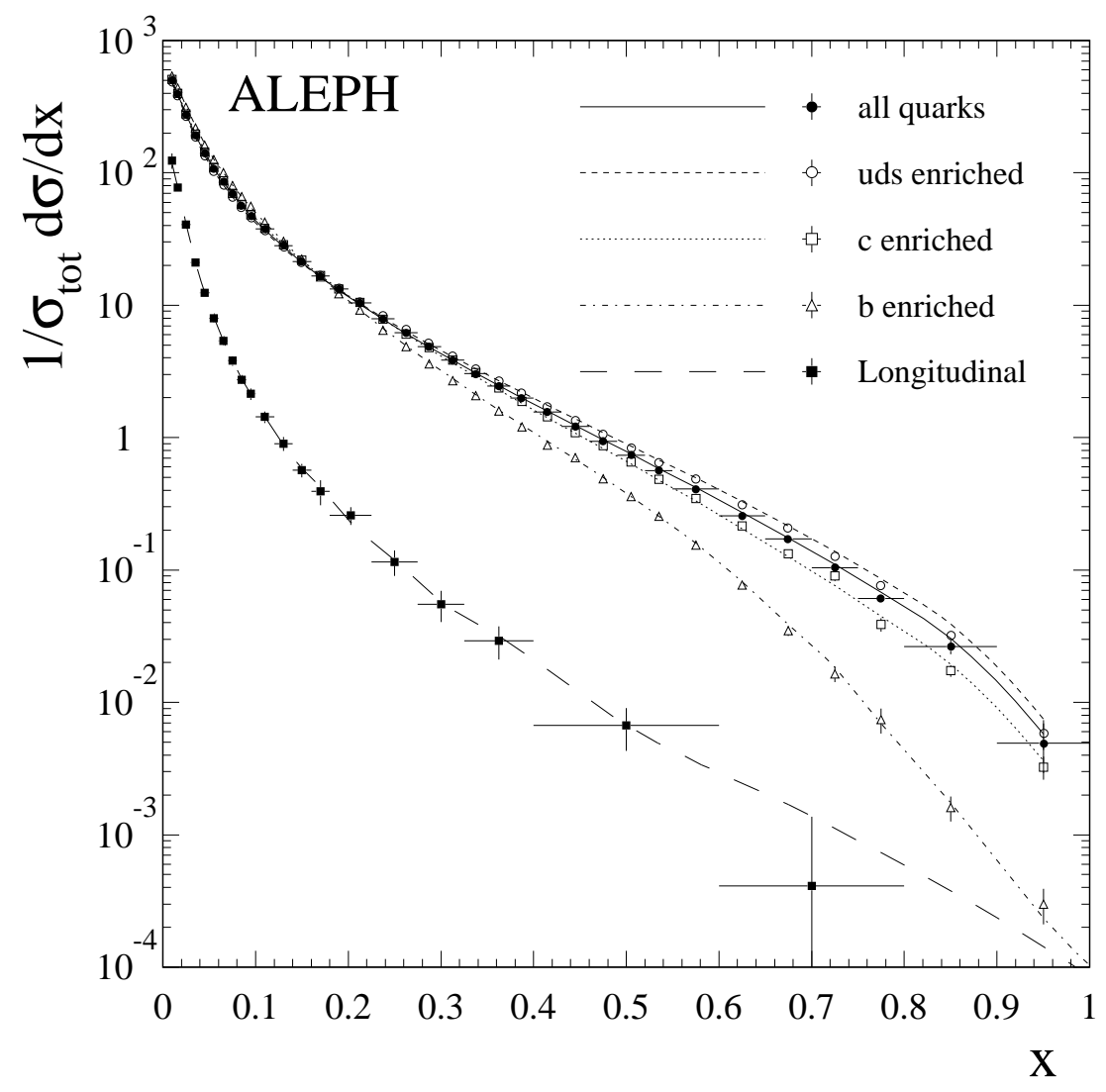

Figure 25: Measured scaledenergy distributions after correction for detector effects (symbols) and comparison with the predictions from JETSET 7.3 (curves). The distributions are normalized to the total number of events. Error bars include statistical and systematic uncertainties. The same binning is used for the inclusive and flavour-tagged distributions.

at a reference energy, $\sqrt{s_{0}}$. Here the index $i$ represents, separately, light, charm and bottom quarks and gluons. The exponential function is motivated by the Modified Leading-Log Approximation (MLLA) [86, 87], which also predicts a single value of the $c$ parameter for all quark flavours as well as the gluon. In total, 13 parameters are used to describe the fragmentation functions at one energy. The evolution to another energy requires two more parameters: $\alpha_{s}$, which determines the perturbative evolution, and $h_{0}$, which parametrizes the non-perturbative effects in the evolution. Finally, the effective leading-order coupling constant $\beta_{s}$ introduced in Eq. (51) is required. In total, there are sixteen parameters, which are all fitted simultaneously to the available data.

An overall fit of the QCD predictions to all ALEPH data and the inclusive data from $\sqrt{s}=22 \mathrm{GeV}$ to $\sqrt{s}=91.2 \mathrm{GeV}$ discussed above is performed. The results are shown in Table 17. Figure 26 shows that the overall agreement between data and prediction is good and that the QCD evolution reproduces the observed scaling violations.

Most of the experimental part of the systematic errors in the $\alpha_{s}$ determination is already contained in the error obtained from the fit. The only remaining uncertainties are from the treatment of the normalization errors $\left(\Delta \alpha_{s}=0.002(\right.$ norm $\left.)\right)$ and the knowledge of the purities of the flavour-enriched samples $\left(\Delta \alpha_{s}=0.004\right.$ (purity)). The total experimental error of $\alpha_{s}\left(M_{Z}\right)$ is $\Delta \alpha_{s}($ exp $)= \pm 0.005$ (fit) $\pm 0.002($ norm $) \pm 0.004$ (purity) $= \pm 0.007$.

Theoretical errors were determined, following [71], by varying independently the factorization and renormalization scales in the range $-1 \leq \ln \left(\mu^{2} / s\right) \leq 1$. The resulting changes in $\alpha_{s}\left(M_{Z}\right)$ are $\Delta \alpha_{s}($ theo $)= \pm 0.002\left(\mu_{R}\right) \pm 0.006\left(\mu_{F}\right)$. Combining all errors in quadrature the final result for $\alpha_{s}\left(M_{Z}\right)$ becomes

$$
\alpha_{s}\left(M_{Z}\right)=0.126 \pm 0.007(\exp ) \pm 0.006(\text { theo })=0.126 \pm 0.009
$$




\begin{tabular}{|c|c|c|c|c|}
\hline \multicolumn{5}{|c|}{$\alpha_{s}\left(M_{Z}\right)=0.1258 \pm 0.0053$} \\
\hline & light $(u d s)$ quarks & $c$ quarks & $b$ quarks & gluons \\
\hline$N$ & $0.372 \pm 0.005$ & $0.359 \pm 0.006$ & $0.295 \pm 0.008$ & $0.395 \pm 0.020$ \\
$a$ & $1.69 \pm 0.04$ & $3.09 \pm 0.16$ & $3.29 \pm 0.09$ & $2.6 \pm 0.8$ \\
$b$ & $-1.40 \pm 0.06$ & $-1.10 \pm 0.09$ & $-1.69 \pm 0.07$ & $-1.59 \pm 0.29$ \\
\cline { 2 - 5 }$c$ & \multicolumn{4}{|c|}{$0.252 \pm 0.014$} \\
\multirow{2}{*}{$\beta_{s}$} & \multicolumn{4}{|c}{$0.199 \pm 0.008$} \\
\hline
\end{tabular}

Table 17: Results of the fit to all data. The parameters $N, a, b$ and $c$ define the shape of the fragmentation functions at the scale $\sqrt{s_{0}}=22 \mathrm{GeV}$. The errors include statistical and experimental systematic uncertainties, except for those related to flavour tagging. There are sizeable correlations amongst most of the parameters, which may be as large as $90 \%$ between the parameters of the fragmentation functions.

The main single contribution to the error on $\alpha_{s}$ comes from the dependence on the factorization scale chosen. The measurement is very independent of the other $\alpha_{s}$ determinations presented here and it agrees well with them.

\subsubsection{Summary of $\alpha_{s}$ measurements}

Table 18 summarizes the $\alpha_{s}$ determinations with the four methods described above. The overall agreement between the results is excellent. The four measurements are obtained from very different processes. The smallest error is provided by the $R_{\tau}$ determination. This is a totally inclusive measurement at low energy, which involves a sum over all hadronic final states and all values of $Q^{2}$ below $m_{\tau}^{2}$. However, the assessment of the theoretical error is still not totally settled (see, for instance, refs. $[56,57,58]$ ). The $\alpha_{s}$ determination based on $R_{l}$ has a large error, but it is very clean theoretically and is limited mainly by statistics. Combining the results of the four LEP experiments decreases the error by almost a factor two. The last two determinations in Table 18 are based on less inclusive processes where some hadronic variables are not summed over and larger theoretical uncertainties result. The event shapes determination is based on infrared-safe observables, for which there is a prediction from perturbative QCD that has to be supplemented by non-perturbative corrections. The result obtained using all-orders resummed predictions has a smaller theoretical error and, at this time, it is regarded as the final ALEPH result coming from event shape variables. The determination from scaling violations is based on the energy evolution of an infrared-sensitive observable and makes use of $e^{+} e^{-}$data at lower centre-of-mass energies. That all these different observables lead to compatible values of $\alpha_{s}\left(M_{Z}\right)$ with a precision of few percent has to be regarded as a major success of QCD.

\subsubsection{The Running of $\alpha_{s}$}

One of the fundamental tests of QCD is to verify that $\alpha_{s}$ measurements taken at different renormalization scales are related by the $\beta$-function of QCD. LEP1 provides two points, $\alpha_{s}\left(M_{\tau}\right)$ 


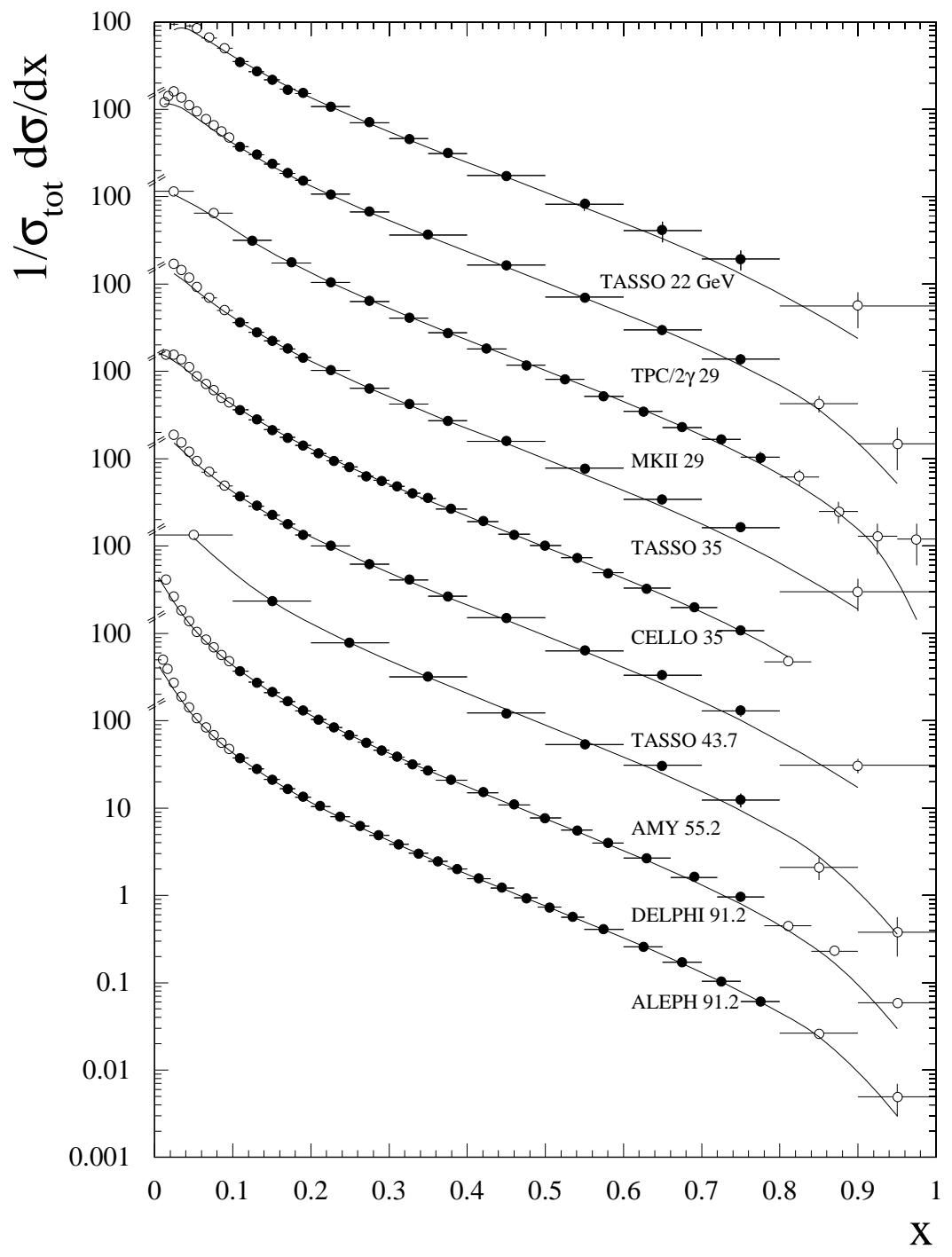

Figure 26: Inclusive allflavour scaledenergy distributions used in the QCD fit. Only the full dots enter the fit. Errors shown include statistical and systematic uncertainties. The curves represent the results of the fit.

\begin{tabular}{|l|c|c|c|}
\hline Method & $Q(\mathrm{GeV})$ & $\alpha_{s}(Q)$ & $\alpha_{s}\left(M_{Z}\right)$ \\
\hline$R_{l}$ & 91.2 & $0.123 \pm 0.007$ & $0.123 \pm 0.007$ \\
$R_{\tau}$ & 1.777 & $0.353 \pm 0.022$ & $0.122 \pm 0.003$ \\
Event shapes & 91.2 & $0.125 \pm 0.005$ & $0.125 \pm 0.005$ \\
Scaling Violations & $22-91.2$ & - & $0.126 \pm 0.009$ \\
\hline
\end{tabular}

Table 18: Summary of $\alpha_{s}$ measurements from ALEPH.

and $\alpha_{s}\left(M_{Z}\right)$. For $\alpha_{s}\left(M_{Z}\right)$ two independent measurements are available, one from $R_{l}$ and one from global event shapes. In order to test that QCD really is the universal theory describing strong interaction processes, further information has to be included.

A comprehensive compilation can be found in [88], from which the non-ALEPH results shown in Table 19 are taken. The various measurements are clearly incompatible with an 


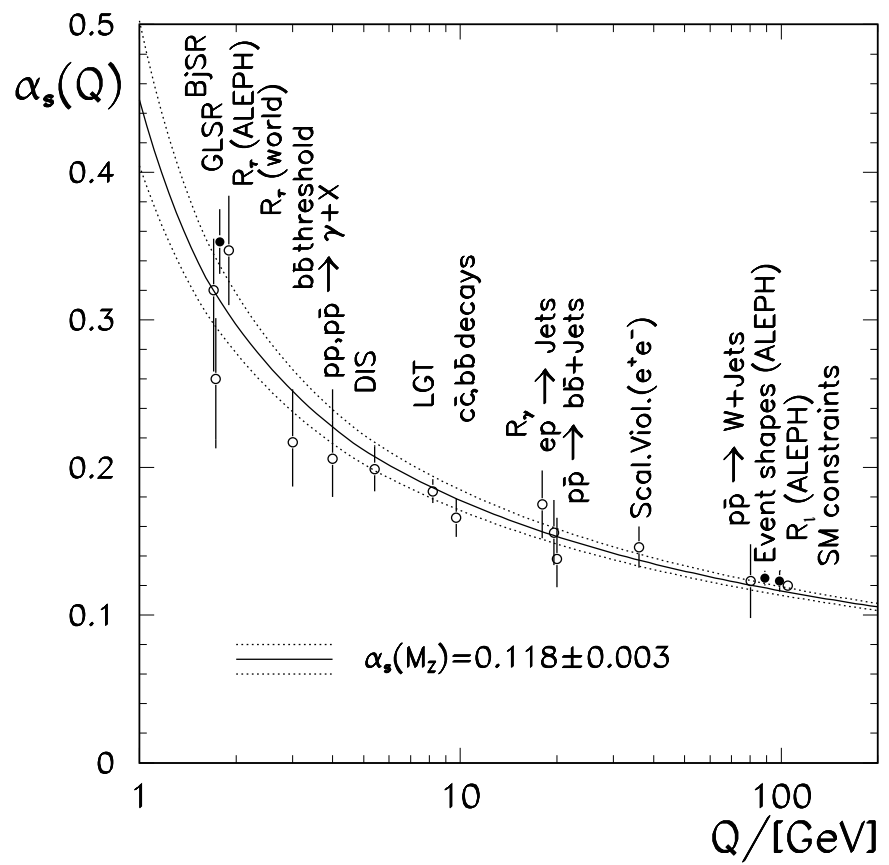

Figure 27: Measurements of the strong coupling constant at various energy scales, full dots representing the ALEPH results. The curves show the QCD prediction for the running with $\alpha_{s}\left(M_{Z}\right)=0.118 \pm 0.003$.

energy independent value of the strong coupling constant. The crucial test for QCD thus boils down to showing that after evolving according to the QCD prediction the individual results to a common reference scale, here taken to be $M_{Z}$, all measurements are compatible with one common value.

The data from Table 19 are displayed in Fig. 27. When evolved up to the $\mathrm{Z}$ mass all measurements are compatible with one common value, which has been chosen as $\alpha_{s}\left(M_{Z}\right)=$ $0.118 \pm 0.003$, the 1996 average done by the Particle Data Group [89]. The data shown in Fig. 27 are compatible with the QCD expectation based on the above average, demonstrating convincingly the running of the strong coupling constant.

\subsection{Angular Dependence of Event Shapes}

In the previous sections neither the experimental distributions nor the theoretical calculations took into account the orientation of the final state with respect to the beam line, measured, for instance, by the polar angle of the thrust axis, $\theta_{T}$. In this section the event orientation is retained and the distributions of the event-shape variables $y_{3}$, defined by the Durham jetfinding algorithm, thrust $(T)$, wide-jet broadening $\left(B_{W}\right)[90]$ and heavy jet mass $\left(M_{H}\right)$ are measured as function of $\cos \theta_{T}$ and compared to QCD predictions. The relationship between event shape and orientation has been investigated previously by experiments at PETRA [91] and LEP [92, 93], using data samples of up to around $10^{5}$ events. The analysis here is based on about 3.6 million events. Whereas the earlier studies compared to predictions computed to $\mathcal{O}\left(\alpha_{s}\right)[91,93]$ or using approximate formulae at $\mathcal{O}\left(\alpha_{s}^{2}\right)[92]$, the analysis here uses predictions based on an integration of the full $\mathcal{O}\left(\alpha_{s}^{2}\right)$ matrix elements using the program EVENT2 [94]. Both the higher statistics measurements and the more accurate theoretical formulae lead to a more stringent test of QCD than was possible in previous analyses.

\section{Event Selection and Theoretical Predictions}

The criteria for track and event selection are described in Section 1.3. Both charged tracks and neutral particles are used and the reconstructed thrust axis is required to be well contained within the detector acceptance, i.e. $\cos \theta_{T} \leq 0.9$. The selection efficiency is $87 \%$, and about 


\begin{tabular}{|l|c|c|c|}
\hline \multicolumn{1}{|c|}{ Measurement } & $\mu(\mathrm{GeV})$ & $\alpha_{s}(\mu)$ & $\alpha_{s}\left(M_{Z}\right)$ \\
\hline Bjorken sum rule & 1.732 & $0.320 \pm 0.035$ & $0.118 \pm 0.005$ \\
Gross-Llewellyn Smith sum rule & 1.732 & $0.260 \pm 0.041$ & $0.110 \pm 0.006$ \\
$R_{\tau}($ A LEPH $)$ & 1.777 & $0.353 \pm 0.022$ & $0.123 \pm 0.003$ \\
$R_{\tau}($ world $)$ & 1.777 & $0.347 \pm 0.037$ & $0.122 \pm 0.005$ \\
$b \bar{b}$ threshold & 3. & $0.217 \pm 0.036$ & $0.110 \pm 0.008$ \\
prompt $\gamma$ & 4.0 & $0.206 \pm 0.042$ & $0.112 \pm 0.012$ \\
deep inelastic scattering & 5.4 & $0.199 \pm 0.016$ & $0.115 \pm 0.005$ \\
lattice gauge theory & 8.2 & $0.184 \pm 0.008$ & $0.117 \pm 0.003$ \\
$c \bar{c}, b \bar{b}$ decays & 9.7 & $0.166 \pm 0.013$ & $0.112 \pm 0.006$ \\
$R_{\gamma}$ & 18.0 & $0.175 \pm 0.023$ & $0.128 \pm 0.012$ \\
$e p \rightarrow$ Jets & 19.6 & $0.156 \pm 0.022$ & $0.119 \pm 0.013$ \\
$p \bar{p} \rightarrow b \bar{b}+$ Jets & 20.0 & $0.138 \pm 0.028$ & $0.109 \pm 0.016$ \\
$e^{+} e^{-}$fragmentation & 36.0 & $0.146 \pm 0.014$ & $0.124 \pm 0.010$ \\
$p \bar{p} \rightarrow$ W+Jets & 80.2 & $0.123 \pm 0.025$ & $0.121 \pm 0.024$ \\
$R_{l}$ (ALEPH) & 91.2 & $0.123 \pm 0.007$ & $0.123 \pm 0.007$ \\
Standard Model fit & 91.2 & $0.120 \pm 0.003$ & $0.120 \pm 0.003$ \\
event shapes (ALEPH) & 91.2 & $0.125 \pm 0.005$ & $0.125 \pm 0.005$ \\
\hline
\end{tabular}

Table 19: Summary of $\alpha_{s}$ measurements, comparing ALEPH results to measurements at different energies and different processes. The compilation of results is taken from [88] where references can be found.

$3.6 \times 10^{6}$ hadronic events recorded in 1991 to 1995 remain for further analysis with a small background of $0.2 \%$ from $\tau^{+} \tau^{-}$events.

The double differential cross section with respect to $\cos \theta_{T}$ and an event-shape variable $y$ is given to $\mathcal{O}\left(\alpha_{s}^{2}\right)$ as

$$
\begin{aligned}
\frac{1}{\sigma} \frac{d^{2} \sigma\left(y, \cos \theta_{T}\right)}{d y d \cos \theta_{T}} & =\frac{\alpha_{s}\left(\mu^{2}\right)}{2 \pi} A\left(y, \cos \theta_{T}\right) \\
& +\left(\frac{\alpha_{s}\left(\mu^{2}\right)}{2 \pi}\right)^{2}\left[A\left(y, \cos \theta_{T}\right) 2 \pi b_{0} \ln \left(\frac{\mu^{2}}{s}\right)+B\left(y, \cos \theta_{T}\right)\right],
\end{aligned}
$$

where $y=y_{3}, 1-T, B_{W}, M_{H}$. The coefficient functions $A\left(y, \cos \theta_{T}\right)$ and $B\left(y, \cos \theta_{T}\right)$ have been computed in [94].

In order to obtain a prediction for the hadron level distributions, the perturbative formula must be modified to account for the effects of hadronization. This is done by multiplying the perturbative prediction bin-by-bin with correction factors derived from the parton and hadron levels of Monte Carlo models. The models used were JETSET [61] version 7.4 (using both the default parton shower option and the $O\left(\alpha_{s}^{2}\right)$ matrix elements), HERWIG [62] version 5.8, 
and ARIADNE [30] version 4.06. Ranges of the event-shape variables are found in which the hadronization corrections are no more than $30 \%\left(0.05<y_{3}<0.22,0.7<T<0.9\right.$, $\left.0.1<B_{w}<0.22,0.12<M_{h}^{2} / s<0.3\right)$. The corrections are only very weakly dependent on $\cos \theta_{T}$.

Detector effects were taken into account by applying bin-by-bin correction factors to the theoretical predictions. Thus the comparison between theory and experiment is done at "detector level". The correction factors $C_{\text {det }}$ are given by the ratio of the corresponding Monte Carlo distributions with and without detector simulation. In the fit ranges used for determining $\alpha_{s}$, the corrections are in the range $0.8<C_{d e t}<1.2$. The correction factors depend on both the event-shape variable and $\cos \theta_{T}$. The $\cos \theta_{T}$ dependence is relatively smooth, however, and shows no particular structure.

\section{Simultaneous Analysis of Event Shapes and Orientation}

Oriented event-shape distributions are measured in nine bins of $\cos \theta_{T}$ between 0.1 and 0.9 . The theoretical prediction of (52) is fitted to the data with $\alpha_{s}\left(M_{Z}^{2}\right)$ as free parameter. The renormalization scale $\mu$ is set to $M_{Z} / 2$. In order to obtain a good description of the data, the two-jet region has to be excluded from the fit, which is based on second order calculations only. In a first step, $\alpha_{s}$ is determined in each bin of $\cos \theta_{T}$. The results obtained are shown in Fig. 28.

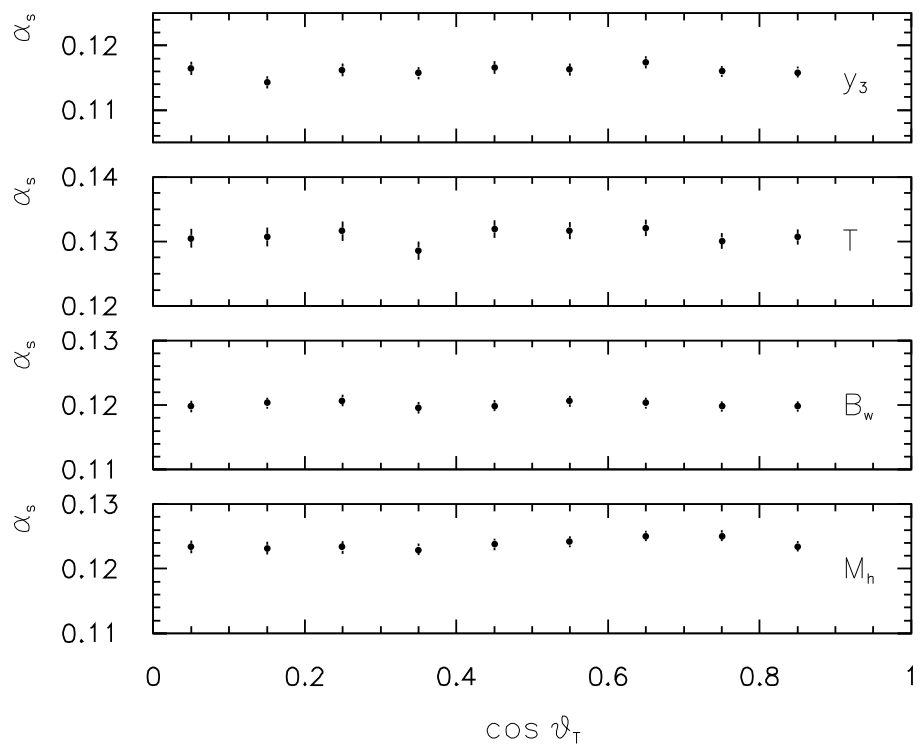

Figure 28: Fitted values of $\alpha_{s}\left(M_{Z}\right)$ in individual bins of $\cos \theta_{T}$ for the event shape variables $y_{3}$, thrust, wide jet broadening, and heavy jet mass. Only the statistical errors are shown.

No systematic dependence on $\cos \theta_{T}$ is found for any variable. Subsequently, the fit is repeated for all bins of $\cos \theta_{T}$ simultaneously. The total $\chi^{2}$ of the simultaneous fit increases less than 10 $\%$ with respect to the $\chi^{2}$ of the fit with individual values of $\alpha_{s}$ in each bin of $\cos \theta_{T}$. The twodimensional distribution can be described by a unique value of $\alpha_{s}$. As an example, Fig. 29 shows the distribution of $y_{3}$, together with the result of the fit, for two different bins of $\cos \theta_{T}$. Both distributions are normalized to unit area. One can clearly see the enhancement in the three-jet region (large $y_{3}$ ) for the angular region perpendicular to the beam line $\left(0.0<\cos \theta_{T}<0.1\right)$.

\section{Event Orientation}

One can also test the relationship between event shape and orientation using the distribution of $\cos \theta_{T}$ for events in a given interval of the event-shape variable. Figure 30(a) shows the 


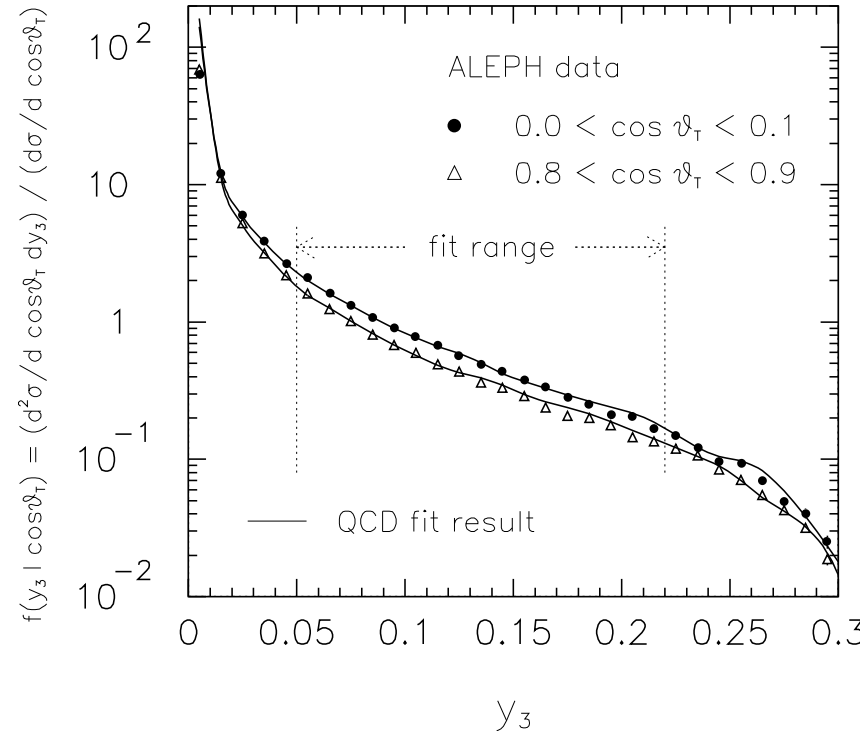

Figure 29: Distribution of $y_{3}$ normalized to unit area for two bins of $\cos \theta_{T}$ shown with the results of the simultaneous fit of the QCD prediction using all $\cos \theta_{T}$ intervals.

distribution of $\cos \theta_{T}$ for events in the two-jet region $(0.9<T<0.99)$, and Fig. 30(b) shows the same for multijet events $(T<0.8)$. The flattening of the distribution for multijets is clearly visible, in good agreement with the QCD prediction (evaluated with $\alpha_{s}=0.117$ ).



Figure 30: Distribution of $\cos \theta_{T}$ for (a) events with $0.9<T<0.99$ and (b) events with $T<0.8$. Fluctuations in the theoretical curves are due to finite Monte Carlo statistics for hadronization and detector corrections.

By integrating over the complete range of the event-shape variable, one obtains the distribution for $\cos \theta_{T}$ for all events. This can be expressed as

$$
\frac{d \sigma}{d \cos \theta_{T}}=\frac{3}{4}\left(1+\cos ^{2} \theta_{T}\right) \sigma_{U}+\frac{3}{2}\left(1-\cos ^{2} \theta_{T}\right) \sigma_{L}
$$


where the longitudinal cross section $\sigma_{L}$ is [95]

$$
\sigma_{L}=2\left(8 \ln \frac{3}{2}-3\right) \sigma_{0} \frac{4}{3} \frac{\alpha_{s}}{\pi}\left(1+l \frac{\alpha_{s}}{\pi}\right)
$$

with $l=0.72$, and the unpolarized transverse cross section is $\sigma_{U}=\sigma_{\text {tot }}-\sigma_{L}$. Fitting this formula to the measured $\cos \theta_{T}$ distribution gives

$$
\sigma_{L} / \sigma=\left(1.22 \pm 0.21_{\text {stat }} \pm 0.11_{\text {syst }}\right) \times 10^{-2},
$$

and $\alpha_{s}\left(M_{Z}\right)=0.121 \pm 0.022$ (stat.) \pm 0.011 (sys.). The relatively large statistical error stems from the fact that the total effect of gluon radiation on the $\cos \theta_{T}$ distribution is small. The systematic error includes experimental uncertainties, estimated by performing the measurement with charged tracks only, hadronization uncertainties, evaluated by using the different models mentioned in Section 2.3, and the scale uncertainty in $\alpha_{s}$. If only the leading order QCD prediction is used, the $\chi^{2}$ of the fit degrades considerably, 85/45, compared with the value obtained with the second order fit, $49 / 44$.

In summary, the analysis of the distributions of event-shape variables and event orientation has shown good agreement with $O\left(\alpha_{s}^{2}\right)$ QCD predictions. For a given event-shape variable, the fitted values of $\alpha_{s}\left(M_{Z}\right)$ are found to be independent of $\cos \theta_{T}$. The large data sample and improved QCD formulae allow for a more stringent test of the theory than previously reported.

\subsection{Test of the Flavour Independence of $\alpha_{s}$}

An important property of QCD is the flavour independence of the strong coupling constant. Results at previous accelerators are consistent with flavour independence [96], although with large uncertainties. Recent results at $Z$ energies have been published in refs. [97].

This analysis compared event-shape-variable distributions for hadronic events with the QCD predictions calculated to second order [59]. The flavour independence is tested by comparing two heavy-flavour samples, one enriched by lepton tag and one by lifetime tag, and a lightflavour sample enriched by lifetime antitag, to the full sample of hadronic events, from which $r^{b}=\alpha_{s}^{b} / \alpha_{s}^{u d s c}$ and $r^{u d s}=\alpha_{s}^{u d s} / \alpha_{s}^{c b}$ are determined. Here, $\alpha_{s}^{i j \ldots}$ is the strong coupling constant between gluons and quarks of flavours $i, j, \ldots$ Details of the analysis can be found in [98]. A summary will be given here.

Out of almost a million hadronic Z decays collected in 1991 and 1992, 40000 are tagged with the lepton-tag method. The ratio

$$
R_{\text {data }}=\frac{\left.\frac{1}{N} \frac{\mathrm{dN}}{\mathrm{dX}}\right|_{b}}{\left.\frac{1}{N} \frac{\mathrm{dN}}{\mathrm{dX}}\right|_{Q \bar{Q}}}
$$

with $\mathrm{X}=$ thrust, $\mathrm{C}$ parameter and differential two-jet rate using both the JADE metric $\left(y_{3}^{J}\right)$ and the Durham metric $\left(y_{3}^{D}\right)$ is measured. Lifetime information obtained mainly with the silicon micro-vertex detector is used to tag about $120000 \mathrm{~b}$ events and $300000 \mathrm{uds}$ events. Then the ratio (53) is measured for both tags and for $\mathrm{X}$ being the differential two jet rate with both the JADE and Durham algorithms.

In order to extract $r^{b}=\alpha_{s}^{b} / \alpha_{s}^{u d s c}$ and $r^{u d s}=\alpha_{s}^{u d s} / \alpha_{s}^{c b}$ from each event-shape variable a $\chi^{2}$ fit of the theoretical expression

$$
R_{t h}(X)=\frac{G^{q, t a g} \cdot f_{t a g}^{q}+G^{q^{\prime}, t a g} \cdot\left(1-f_{\text {tag }}^{q}\right)}{G^{q, Q \bar{Q}} \cdot f_{Q \bar{Q}}^{q}+G^{q^{\prime}, Q \bar{Q}} \cdot\left(1-f_{Q \bar{Q}}^{q}\right)}
$$


is performed to the measured ratio (53). Here the fractions $f_{\text {tag }}^{q}$ and $f_{Q \bar{Q}}^{q}$, respectively, denote the purities of the tagged quark type $q$ in the tagged and the corresponding untagged hadronic sample. The function $G$ includes the theoretical prediction for the distribution of $X$ as well as all the corrections in order to compare it directly with uncorrected data:

$$
G^{q, \mathcal{S}}\left(X_{i}\right)=\sum_{j} M_{d e t}^{q}\left(X_{i}, X_{j}\right) \cdot V_{c u t}^{q, \mathcal{S}}\left(X_{j}\right) \cdot V_{Q E D}^{q}\left(X_{j}\right) \cdot V_{h a d}^{q}\left(X_{j}\right) \cdot F^{q}\left(X_{j}\right),
$$

where

$$
F^{q}\left(X_{j}\right)=\frac{\sigma_{0}^{q}}{\sigma_{T}^{q}} \cdot\left[\frac{\alpha_{s}^{q}(\mu)}{2 \pi} A\left(X_{j}\right) \cdot V_{m a s s}^{q}+\left(\frac{\alpha_{s}^{q}(\mu)}{2 \pi}\right)^{2}\left(A\left(X_{j}\right) \cdot V_{m a s s}^{q} 2 \pi b_{0} \ln \frac{\mu^{2}}{M_{Z}^{2}}+B\left(X_{j}\right)\right)\right],
$$

with $\sigma_{0}^{q}$ the Born-level cross section for massless quarks of type $q$ and $\sigma_{T}^{q}$ the total cross section including mass effects[99]. Here $\mathcal{S}$ stands for any sample, tag or untagged. $F^{q}\left(X_{j}\right)$ is the second order QCD prediction including mass effects to $\mathcal{O}\left(\alpha_{s}\right)$ and the terms in the expression for $G$ correspond to corrections for detector resolution, selection cuts, QED initial state radiation and hadronization, respectively.

The results of the fits can be found in Table 20. The quality of the fits is good as can be seen,

\begin{tabular}{|c|cccc|cc|}
\cline { 2 - 7 } \multicolumn{1}{c|}{} & \multicolumn{4}{c|}{ lepton tag } & \multicolumn{2}{c|}{ lifetime tag } \\
\multicolumn{1}{c|}{} & Thrust & C param. & $y_{3}^{J}$ & $y_{3}^{D}$ & $y_{3}^{J}$ & $y_{3}^{D}$ \\
\hline$r^{b}$ & 0.993 & 0.969 & 1.027 & 1.014 & 1.024 & 1.033 \\
Stat. err. & \pm 0.011 & \pm 0.013 & \pm 0.014 & \pm 0.014 & \pm 0.008 & \pm 0.009 \\
Syst. err. & \pm 0.019 & \pm 0.020 & \pm 0.032 & \pm 0.030 & \pm 0.037 & \pm 0.031 \\
\hline$r^{\text {uds }}$ & - & - & - & - & 0.974 & 0.968 \\
Stat. err. & - & - & - & - & \pm 0.011 & \pm 0.012 \\
Syst. err. & - & - & - & - & \pm 0.023 & \pm 0.022 \\
\hline
\end{tabular}

Table 20: Results for the determination of $r^{b}$ and $r^{u d s}$ for each method used.

for example, for $y_{3}^{D}$ with the lepton $b$-tag in Fig. 31. The main contributions to the systematic error have a theoretical origin: they come from the uncertainty in the mass corrections, in the hadronization corrections and in the renormalization scale.

Combining the different variables and tags, taking into account their correlations, leads to the final result:

$$
\begin{gathered}
\left.r^{b}=\frac{\alpha_{s}^{b}}{\alpha_{s}^{u d s c}}=1.002 \pm 0.009(\text { stat. }) \pm 0.005(\text { syst. }) \pm 0.021 \text { (theo. }\right) \\
\left.r^{u d s}=\frac{\alpha_{s}^{u d s}}{\alpha_{s}^{c b}}=0.971 \pm 0.009(\text { stat. }) \pm 0.011 \text { (syst. }\right) \pm 0.018(\text { theo. }),
\end{gathered}
$$

which is consistent with the flavour independence of the strong coupling constant.

\subsection{Colour Factors of QCD}

Measurements of the colour factors of QCD can be used to verify that the dynamics is described by an unbroken SU(3) gauge symmetry. The static quark model describes hadrons as bound 


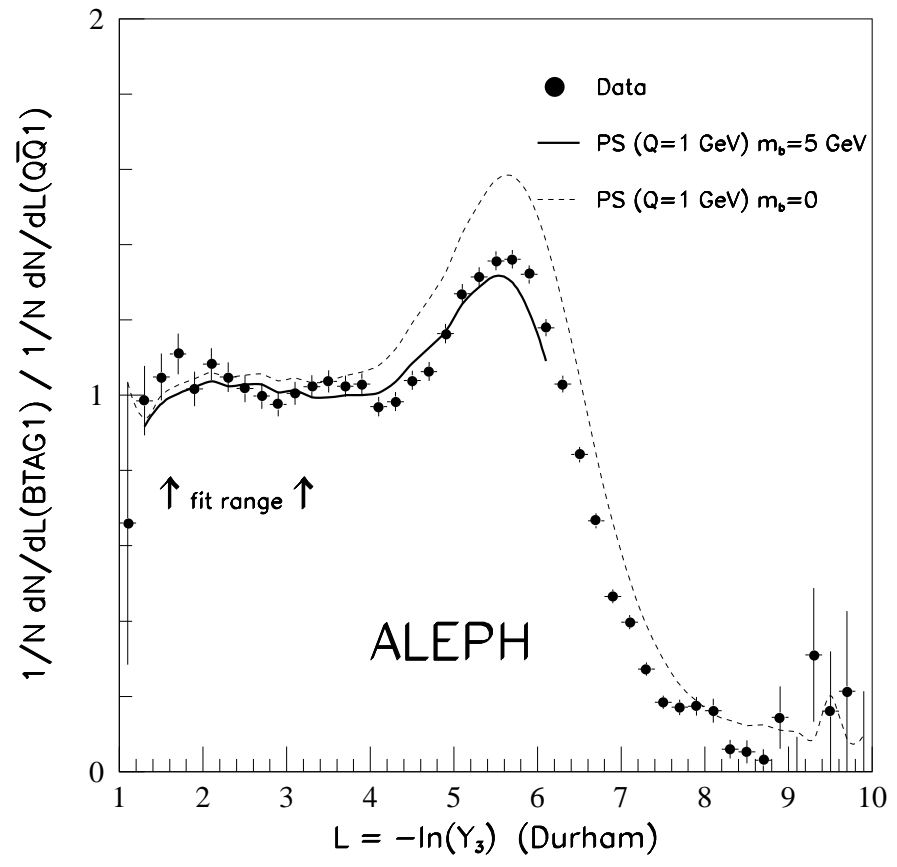

Figure 31: Ratio of the normalized cross section of the $b$-enriched sample tagged with high- $p_{\perp}$ lepton and the full hadronic sample. The full circles are the data, the solid line represents the fit result and the dashed line represents the theoretical prediction without the corrections for the finite mass of the $b$ quark.

states of quarks with three colour degrees of freedom. Assuming that these colours exhibit an $\mathrm{SU}(3)$ symmetry the model is able to explain the observed hadrons as colour singlet systems. Up to this point the concept of colour is just a label, introduced in order to solve the spinstatistics problem for baryons made out of three identical quarks. It has nothing to do with the charge of an interaction. Although it is a natural next step to assume that those colours also govern the dynamics of strong interactions, i.e. building QCD on the gauge group SU(3), this must be tested. It is conceivable, for example, that not all colour degrees of freedom of the quarks contribute to the dynamics of QCD. In this case $\mathrm{SU}(2), \mathrm{SO}(2)$ or $\mathrm{U}(1)$ become possible candidates for the gauge symmetry. Going one step further one can also imagine strong interactions to be described by a spontaneously broken SU(3) symmetry. The resulting massive gauge bosons would result in a dynamical structure which deviates from the SU(3) expectation. Deviations also can be caused by the existence of new physics, which couples to the strong interactions sector. An example for the latter is the case of a light gluino, the supersymmetric partner of the gluon, which at $\mathcal{O}\left(\alpha_{s}^{2}\right)$ contributes three additional fermionic degrees of freedom to the running of $\alpha_{s}$ in $e^{+} e^{-}$-annihilation processes.

The colour factors are defined in Section 1.1. Absorbing the factor $C_{F}$ into the definition of the coupling constant, the theoretical prediction for any physical observable $\sigma$ can be written as

$$
\sigma=F\left(\alpha_{s} C_{F}, \frac{C_{A}}{C_{F}}, n_{f} \frac{T_{F}}{C_{F}}\right)
$$

The colour factors for the case of an arbitrary gauge group are defined as quadratic invariants of the respective generators, in exactly the same way as the ones for QCD. Based on an SU(3) symmetry, QCD predicts $C_{A} / C_{F}=9 / 4$ and $T_{F} / C_{F}=3 / 8$ for all experimental observables. If QCD had to be extended by new physics, deviations from this prediction would be observed. The size of those deviations would depend both on the particular observables under study and 
on the nature of the new interactions.

Three studies have been made in ALEPH. The first one looks at the characteristics of fourjet events. The triple gluon vertex, only present in non-abelian theories, contributes to this process already at tree level. It also contributes to the production of two- and three-jet events, although only in loops. However, the larger sample for these events can compensate their smaller sensitivity. Finally, the running of $\alpha_{s}$ with energy is affected by the colour factors. By studying the measurements of $\alpha_{s}$ presented in Section 3.2 both at the Z resonance and from $\tau$ decays, deviations from the expected running of $\alpha_{s}$ can be investigated.

\subsubsection{Determination using four-jet events}

Diagrams involving the triple gluon vertex contribute to the four-jet cross section at tree level (Fig. 32). A perturbative calculation in $\mathcal{O}\left(\alpha_{s}^{2}\right)$ has been performed by several authors $[100,16]$. The latter [16] is the basis for the matrix element option in the JETSET Monte Carlo. The five-fold differential four-jet cross sections factorize into kinematical and gauge group dependent terms:

$$
\begin{array}{r}
d \sigma^{(4) \propto\left(\frac{\alpha_{s} C_{F}}{\pi}\right)^{2}\left[A\left(y_{i j}\right)\right.}+\left(1-\frac{1}{2} \frac{C_{A}}{C_{F}}\right) B\left(y_{i j}\right)+\left(\frac{C_{A}}{C_{F}}\right) C\left(y_{i j}\right) \\
\left.+\left(n_{f} \frac{T_{F}}{C_{F}}\right) D\left(y_{i j}\right)+\left(1-\frac{1}{2} \frac{C_{A}}{C_{F}}\right) E\left(y_{i j}\right)\right]
\end{array}
$$

where $y_{i j}=m_{i j}^{2} / \mathrm{s}$ denotes the scaled invariant mass squared of any pair of partons $i$ and $j$ with $i, j=1 \ldots 4$ and $n_{f}$ the number of active flavours. The analytical form of the kinematical functions $A \ldots E$, can be found in Ref. [16]. The functions $A$ and $B$ are the contributions from double-bremsstrahlung diagrams (a) and (b) in Fig. 32, C contains all contributions from the triple gluon vertex including interference terms between (c) and double bremsstrahlung diagrams. The functions $D$ and $E$ describe four-fermion final states (d).

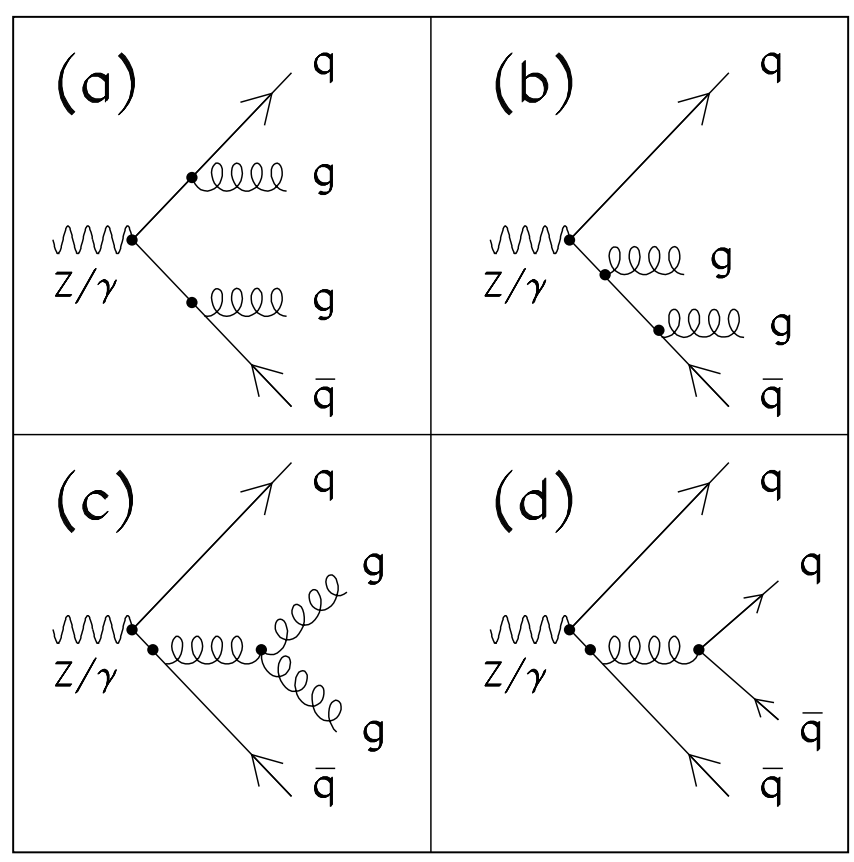

Figure 32: Classes of diagrams contributing to the four-jet cross section in second order QCD.

The ALEPH analysis which has been published in Ref. [101] is based on a maximum likelihood fit of the selected four-jet events to the theoretical prediction (55). 
Using the PTCLUS clustering algorithm [102] for charged and neutral particles, 4148 fourjet events were found in the 1989 and 1990 data samples for which the minimal scaled invariant mass squared of all pairs of clusters $i$ and $j$ fulfilled $\min \left(y_{i j}\right)>y_{\text {cut }}=0.03$. Using jets reconstructed with the PTCLUS algorithm, this value for $y_{c u t}$ was found to result in the best compromise between high statistics and small hadronization corrections resulting from a clean separation of the four jets. In addition the angle to the beam axis for each jet is required to be above 20 degrees, the number of charged tracks or neutrals per jet at least two and the sum of the six scaled invariant masses squared above 0.95. Finally, before comparison to the theoretical prediction, the $y_{i j}$ are rescaled such that the sum becomes unity, i.e. to fulfil momentum conservation for four massless partons.

The colour factors are determined from the data by a maximum likelihood fit of the second order theoretical prediction, i.e. by maximizing

$$
\ln \mathcal{L}=\sum_{i} \ln \frac{\bar{\sigma}_{i}\left(C_{A} / C_{F}, T_{F} / C_{F}\right)}{\bar{\sigma}_{\text {tot }}\left(C_{A} / C_{F}, T_{F} / C_{F}\right)}
$$

with respect to $C_{A} / C_{F}$ and $T_{F} / C_{F}$. The sum runs over all selected four-jet events. For each event $\bar{\sigma}_{i}$ denotes the folded four-jet cross section obtained by summing over all permutations of parton-type assignments to the jets, thereby taking into account that no identification is made of parton type or quark flavour, and $\bar{\sigma}_{t o t}$ is the corresponding total cross section. The ratio $\bar{\sigma}_{i} / \bar{\sigma}_{\text {tot }}$ is the probability density to observe the given set of five kinematical variables $y_{j k}$ in a particular event $i$ as function of the colour factors.

After the fit the results were corrected for detector resolution and fragmentation effects. The corrections were determined from the Lund matrix element model, where the shifts between parton level and final measurements were mapped as function of the colour factors by reweighting a set of Monte Carlo events that were passed through the full detector simulation and analysis chain.

The final result is

$$
\begin{aligned}
& C_{A} / C_{F}=2.24 \pm 0.32_{\text {stat }} \pm 0.25_{\text {syst }} \\
& T_{F} / C_{F}=0.58 \pm 0.17_{\text {stat }} \pm 0.21_{\text {syst }}
\end{aligned}
$$

The systematic error comes mainly from fragmentation uncertainties and unknown higher order corrections, estimated from the JETSET model. The result is shown in Fig. 33 in the twodimensional plot of $T_{F} / C_{F}$ versus $C_{A} / C_{F}$ including its $68 \%$ confidence level contour. The result is in agreement with QCD. Any Abelian theory $\left(C_{A}=0\right)$ is ruled out with more than five standard deviations.

\subsubsection{Determination using two- and three-jet events}

The differential cross section for three jet production can be expressed in terms of normalized momenta $x_{i}=2 p_{i} / \sqrt{s}$. The leading order matrix element is given by Eq. (16). In second order QCD, the colour factor ratios $T_{F} / C_{F}$ and $C_{A} / C_{F}$ contribute to three-parton final states via loop corrections. The corresponding negative divergences are cancelled by adding infrared divergent four-parton final states with small invariant masses. The contributions both from three-parton final states and four-parton final states were calculated using the program EVENT, which is the numerical basis of [59] and is derived from the ERT matrix elements [16]. Since no jet tagging was done, the $x_{i}$ variables are ordered, $x_{1} \geq x_{2} \geq x_{3}$. Taking $x_{1}$ and $x_{2}$ as the independent 


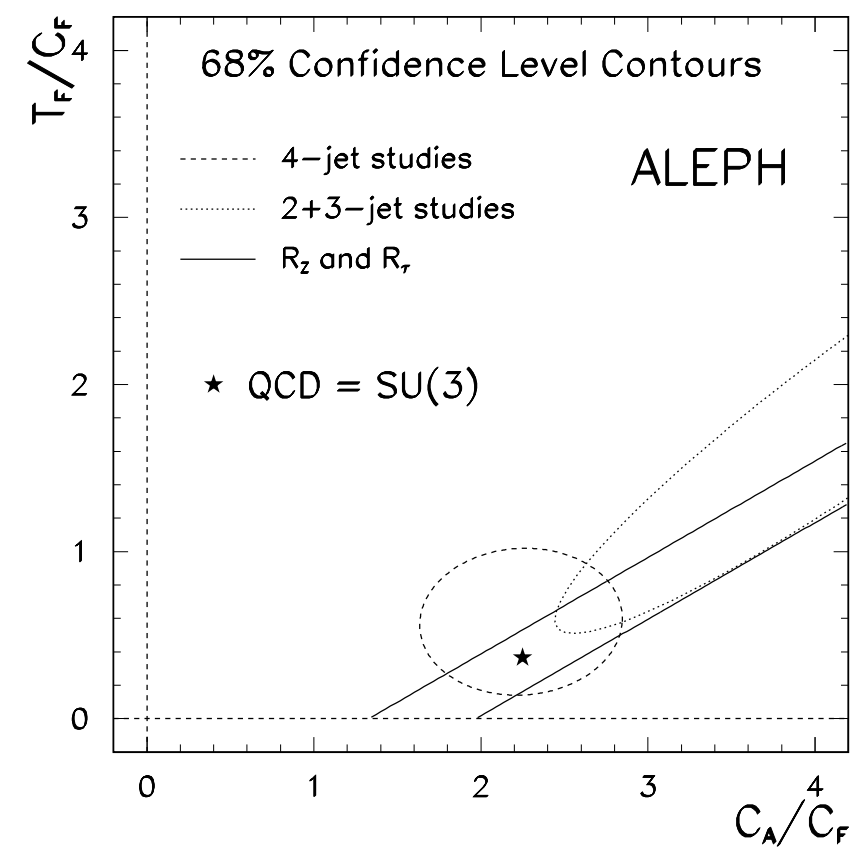

Figure 33: $68 \%$ confidence level contours of $T_{F} / C_{F}$ versus $C_{A} / C_{F}$ as measured from various sources.

variables one obtains a triangular region for the three-jet phase space. This is changed into a rectangular region by the transformation

$$
\begin{array}{ll}
L_{x}=-\ln \left(3-3 x_{1}\right) & 0 \leq L_{x} \leq 1.715 \\
L_{y}=-\ln \left(\frac{3}{4}-\frac{1}{2}\left(\frac{3 x_{2}-2}{3 x_{1}-2}\right)\right) & 0 \leq L_{y} \leq 1.386
\end{array} .
$$

Here the logarithms reduce the strong variation of the function (16). The upper limits are determined by the $y_{c u t}$ value chosen to select three-jet events for this analysis.

For jet finding, the Durham algorithm with the E0 recombination scheme has been used with a cutoff $y_{c u t}=0.06$. This choice is fairly safe against much dependence on hadronization effects and the renormalization scale and yields a sufficiently large number of three-jet events.

The final three-jet cross section can be expressed in terms of fixed kinematical integrals $I$ over each bin $k l$ of the phase space $\left(L_{x}, L_{y}\right)$ and the gauge couplings $\left(\alpha_{s} C_{F}, T_{F} / C_{F}, C_{A} / C_{F}\right)$ :

$$
\frac{1}{\sigma_{\text {tot }}} \frac{d^{2} \sigma^{(3)}}{d L_{x}^{k} d L_{y}^{l}}=\frac{\alpha_{s} C_{F}}{2 \pi} I_{0}^{k l}+\left(\frac{\alpha_{s} C_{F}}{2 \pi}\right)^{2}\left[I_{1}^{k l}-I_{0}^{k l}\left(\frac{3}{2}-\frac{b_{0}}{2} \ln f\right)+\frac{T_{F}}{C_{F}} n_{f} I_{2}^{k l}+\frac{C_{A}}{C_{F}} I_{3}^{k l}\right]
$$

Here $f$ denotes the scale factor $f=\mu^{2} / M_{Z}^{2}$ and $b_{0}$ the leading order coefficient of the QCD $\beta$ function in its colour factor decomposition, as given in the appendix, Eq.(90). The strong coupling constant $\alpha_{s}$ has to be evaluated at the renormalization scale $\mu$.

In second order perturbation theory the two-jet rate is determined as the complement to the three-jet and four-jet rate:

$$
\frac{\sigma_{2}}{\sigma_{t o t}}=1-\frac{\sigma_{3}}{\sigma_{t o t}}-\frac{\sigma_{4}}{\sigma_{t o t}} .
$$

Here the sensitivity to the gauge structure comes from the $\mathcal{O}\left(\alpha_{s}^{2}\right)$ corrections to the three- and four-jet rates. 


\section{Data analysis and results}

From the 1992 data, 614478 hadronic events have been selected using charged tracks and neutral objects from the energy flow analysis. The event and charged track selection used the standard criteria. For the final event selection, the total visible energy $E_{v i s}$ was required to be in excess of $0.5 \sqrt{\mathrm{s}}$ and the momentum imbalance of all accepted tracks and objects along the beam direction smaller than $40 \%$ of the visible energy.

Furthermore, cuts on jet reconstruction quality have been applied. The angle between the jet axis and the beam axis must lie between 20 and 160 degrees. There must be at least two tracks or neutral objects in a jet. The sum of the normalized invariant masses $y_{i j}$ must be greater than 0.95. These cuts yield a final sample of 111041 three-jet events and a two-jet rate of $\sigma_{2} / \sigma_{\text {tot }}=0.801$. In order to compare the data jets with ideal three-parton events, the normalized invariant masses $y_{i j}$ are scaled to fulfil $\sum_{i j} y_{i j}=1$.

For the fit procedure, the theoretical prediction Eq.(57) is transformed to detector level using the Monte Carlo probabilities for a three-parton event in bin $k l$ at parton level to migrate to bin ij at detector level. The background from genuine two- and four-parton events which enter the three-jet sample is added. The measured two-jet rate is corrected to parton level and compared to the prediction Eq.(58).

The systematic uncertainties of this analysis were determined by varying the renormalization scale over the range $M_{Z} / 2<\mu<2 M_{Z}$ and by changing the jet quality cuts such that the accepted three-jet rate varies by $10 \%$. In addition, the sensitivity to two- and four-jet background in the three-jet sample and the uncertainties in the hadronization corrections were studied by using the HERWIG 5.4 and the ARIADNE 4.4 models instead of the JETSET 7.3 parton shower model. An estimate of the importance of higher order corrections was obtained by using the JETSET 7.3 parton shower model with the parton level defined by the four partons with the highest virtuality, thus effectively removing the effect of branchings beyond those allowed by an $\mathcal{O}\left(\alpha_{s}^{2}\right)$ calculation. Finally, the systematic error associated with the kinematic rescaling was taken to be the difference between the nominal scaling of the $y_{i j}$ and the result of the reconstruction of the kinematics when projecting all jets into the event plane.

The information from these systematic variations around the nominal analysis has been converted into a covariance matrix for the systematic error:

$$
C_{i j}=\sum_{k=1}^{n} \Delta\left(x_{i}\right)_{k} \Delta\left(x_{j}\right)_{k},
$$

where $\Delta\left(x_{i}\right)_{k}$ is the change in $x_{i} \in\left\{\alpha_{s} C_{F}, T_{F} / C_{F}, C_{A} / C_{F}\right\}$ compared to the nominal analysis under the systematic variation of $k$. For the final result the systematic covariance matrix thus obtained was added to the covariance matrix of the statistical errors.

The dominant error for the combined result comes from hadronization, which contributes roughly twice as much as the statistical error. Next largest is the error from the renormalization scale variation (for the colour factors) and the error from kinematical rescaling (for $\alpha_{s} C_{F}$ ). The errors arising from background and experimental cuts are comparatively small.

The combined measurements from two- and three-jet events yield the result:

$$
\begin{aligned}
& \alpha_{s} C_{F}=0.210 \pm 0.016_{\text {stat }} \pm 0.048_{\text {syst }} \\
& C_{A} / C_{F}=4.49 \pm 0.75_{\text {stat }} \pm 1.12_{\text {syst }} \\
& T_{F} / C_{F}=2.01 \pm 0.49_{\text {stat }} \pm 0.86_{\text {syst }}
\end{aligned}
$$


where the errors quoted are the statistical and the systematic uncertainties. The results are highly correlated, with correlation coefficients $\rho\left(\alpha_{s} C_{F}, T_{F} / C_{F}\right)=0.761, \rho\left(\alpha_{s} C_{F}, C_{A} / C_{F}\right)=$ 0.539 and $\rho\left(C_{A} / C_{F}, T_{F} / C_{F}\right)=0.956$. From the total uncertainties the $\chi^{2}$ probability of the $\mathrm{SU}(3)$ expectation for $n_{f}=5$ is $52 \%$. The result is shown in Fig. 33. Abelian groups with $C_{A}=0$ and $T_{F}>0$ are only consistent with the measurement at a confidence level below $2 \times 10^{-6}$.

\subsubsection{Information from the running of $\alpha_{s}$}

ALEPH measurements of the strong coupling constant $\alpha_{s}$ both on the $\mathrm{Z}$ resonance and from $\tau$ decays have been presented in Section 3.2. The consistency of these measurements with the running expected from QCD over this large energy range provides a powerful constraint on the dynamical structure of the theory of strong interactions.

The running of the strong coupling constant is a function of the gauge structure of the theory of strong interactions and thus allows constraints to be placed on the colour factor ratios. However, a consistent analysis cannot be based directly on available measurements of $\alpha_{s}$, because those usually are determined under the assumption that QCD is based on an $\mathrm{SU}(3)$ symmetry. To probe the gauge structure itself, thus also requires a reevaluation of $\alpha_{s}$ at different energy scales. For many of the rather involved measurements of the strong coupling constant this is not easily achieved. Exceptions are the measurements of $\alpha_{s}$ from $R_{l}$ and $R \tau$. These are conceptually very simple and span a very large energy range.

The theoretical predictions for $R_{l}$ and $R_{\tau}$ for arbitrary gauge groups are described in Appendix A. In this evaluation the evolution equations for the coupling constant and quark masses were integrated numerically. This ensures that all terms are retained which in principle are known.

The analysis was performed as follows: For a given set of colour factor ratios $(x, y)$ the strong coupling constant $a \equiv a\left(M_{Z}\right)=\left(\alpha_{s}\left(M_{Z}\right) C_{F}\right) /(2 \pi)$ was determined by minimizing

$$
\chi_{e x p}^{2}=\frac{\left(R_{l}^{o b s}-R_{l}(a, x, y)\right)^{2}}{\sigma^{2}\left(R_{l}\right)}+\frac{\left(R_{\tau^{o b s}}-R_{\tau}(a, x, y)\right)^{2}}{\sigma^{2}\left(R_{\tau}\right)}
$$

with respect to $a$. Here the errors $\sigma\left(R_{l}\right)$ and $\sigma\left(R_{\tau}\right)$ are the purely experimental errors of the two measurements, i.e. the value of $a$ is determined independently of all assumptions about the theoretical uncertainties and the $\chi^{2}$ has a well defined statistical meaning. In a second step, the theoretical uncertainties are taken into account. Now $a$ is kept fixed at the fitted value and a covariance matrix is determined for the theoretical uncertainties of $R_{l}$ and $R_{\tau}$.

The theoretical prediction depends on a set of $n$ independent parameters $p_{k}$ with uncertainties $\Delta p_{k}$. The resulting covariance matrix for $R_{l}$ and $R_{\tau}$ then is given by

$$
C_{i j}(R)=\sum_{k=1}^{n} \Delta\left(R_{i}\right)_{k} \Delta\left(R_{j}\right)_{k}
$$

where $\Delta\left(R_{i}\right)_{k}$ is the change in $R_{i}$ when varying the parameter $p_{k}$ by $\Delta p_{k}$.

The relevant parameters and their uncertainties are listed in Table 21. For the quark masses the $\overline{\mathrm{MS}}$ running masses are related to the pole masses by Eq.(93) in Appendix A. Following [103] the pole mass can be identified with the constituent mass of the quarks, which leads to the values given in Table 21. The value for the mass of the top quark is taken from the direct measurement [104]. For the determination of the theoretical error for $R_{l}$ and $R_{\tau}$ the "light" 
quark masses $u, d, s, c$ and $b$ have to be varied coherently, because their uncertainty is mainly due to the limited understanding of bound-state effects in QCD. The errors for the top quark and the Higgs mass are varied independently.

The uncertainties associated with the finite order of the perturbative expansion can be estimated by explicit assumptions about the size of a next order coefficient, or, alternatively, by varying the highest order coefficient that is available around its nominal value. Here the latter approach is taken with an error estimate equal to the full size of the SU(3) prediction. The 3rd order coefficients $K_{3}, R_{3}$ and $T_{3}$ are considered to be fully correlated. The non-perturbative correction $\delta_{N P}$ to $R_{\tau}$ was varied by $\pm 200 \%$ around its central value.

\begin{tabular}{|c||c|c|}
\hline Parameter & Central Value & Uncertainty \\
\hline \hline$M_{u}, M_{d}$ & $0.3 \mathrm{GeV} / c^{2}$ & $\pm 0.2 \mathrm{GeV} / c^{2}$ \\
$M_{s}$ & $0.5 \mathrm{GeV} / c^{2}$ & $\pm 0.2 \mathrm{GeV} / c^{2}$ \\
$M_{c}$ & $1.6 \mathrm{GeV} / c^{2}$ & $\pm 0.3 \mathrm{GeV} / c^{2}$ \\
$M_{b}$ & $4.7 \mathrm{GeV} / c^{2}$ & $\pm 0.5 \mathrm{GeV} / c^{2}$ \\
\hline$M_{t}$ & $180 \mathrm{GeV} / c^{2}$ & $\pm 12 \mathrm{GeV} / c^{2}$ \\
\hline$M_{H}$ & $300 \mathrm{GeV} / c^{2}$ & $\pm 240 \mathrm{GeV} / c^{2}$ \\
\hline$g_{1}$ & Eq. $(91)$ & \pm 15.5 \\
\hline$b_{2}$ & Eq. $(90)$ & \pm 19.0801 \\
\hline$K_{3}$ & Eq. $(94)$ & \pm 2.2996 \\
$R_{3}$ & Eq. $(95)$ & \pm 40.789 \\
$T_{3}$ & Eq. $(96)$ & \pm 4.183 \\
\hline$\delta_{c}$ & -0.011 & \pm 0.022 \\
\hline
\end{tabular}

Table 21: Uncertainties assumed for the input parameters to the theoretical prediction of $R_{l}$ and $R_{\tau}$. Independent parameters are separated by a horizontal line. For correlated parameters the correlation is assumed to be $100 \%$. Equations (90-96) can be found in the Appendix.

Using the values given in Table 21 for the uncertainties in the parameters determining the theoretical prediction, the $\Delta\left(R_{i}\right)$ were taken to be half the range covered when varying the corresponding parameter up and down by its error. Having thus determined a covariance matrix due to theoretical uncertainties, theoretical and experimental errors were added in quadrature and the $\chi^{2}$ reevaluated. Confidence levels are based on this $\chi^{2}$ value.

The experimental results from ALEPH, $R_{\tau}=3.645 \pm 0.024$ [54] and $R_{l}=20.746 \pm 0.073$ [106] have been analyzed in terms of colour factor ratios. The energy dependent strong coupling constant was fitted for any pair of colour factor ratios $\left(C_{A} / C_{F}, T_{F} / C_{F}\right)$ and the best fit $\chi^{2}$ used as an indicator whether or not that particular combination is compatible with the experimental data.

The resulting confidence contour in the colour factor plane is displayed in Fig. 33. Within the experimental and theoretical uncertainties QCD is perfectly compatible with the data.

\subsubsection{Summary}

The combined results from two-, three- and four-jet events and from the running of $\alpha_{s}$ are:

$$
C_{A} / C_{F}=2.47 \pm 0.31
$$




$$
T_{F} / C_{F}=0.52 \pm 0.19
$$

with a correlation of $\rho\left(C_{A} / C_{F}, T_{F} / C_{F}\right)=0.86$. This is in good agreement with the QCD prediction $C_{A} / C_{F}=2.25$ and $T_{F} / C_{F}=0.375$. The resulting confidence contour in the colour factor plane is shown in Fig. 34 together with the expectations for all simple Lie groups. Abelian theories with $C_{A}=0$ are excluded by eight standard deviations.

As a possible extension of QCD, models with light gluinos have been discussed [105]. Since a gluino is a fermion with three times the colour charge of a quark, at leading order the number of active quark flavours $n_{f}$ has to be replaced by $\left(n_{f}+3 n_{g}\right)$, with $n_{g}$ the number of light gluinos. For $n_{f}=5$ and $n_{g}=1$ the effective colour factor ratio would be

$$
\left(\frac{T_{F}}{C_{F}}\right)^{\mathrm{eff}}=1.6\left(\frac{T_{F}}{C_{F}}\right)^{\mathrm{QCD}}=0.6
$$

while $C_{A} / C_{F}$ would still be 2.25 . This model is excluded by the experimental result with $93 \%$ confidence level.

The $T_{F} / C_{F}$ measurement can be interpreted as a determination of the number of gluons $N_{A}$. Assuming $N_{C}=3$ colours for quarks, one has

$$
N_{A}=\frac{N_{C}}{T_{F} / C_{F}}=5.8 \pm 2.1
$$

compatible with the expectation $N_{A}=8$.

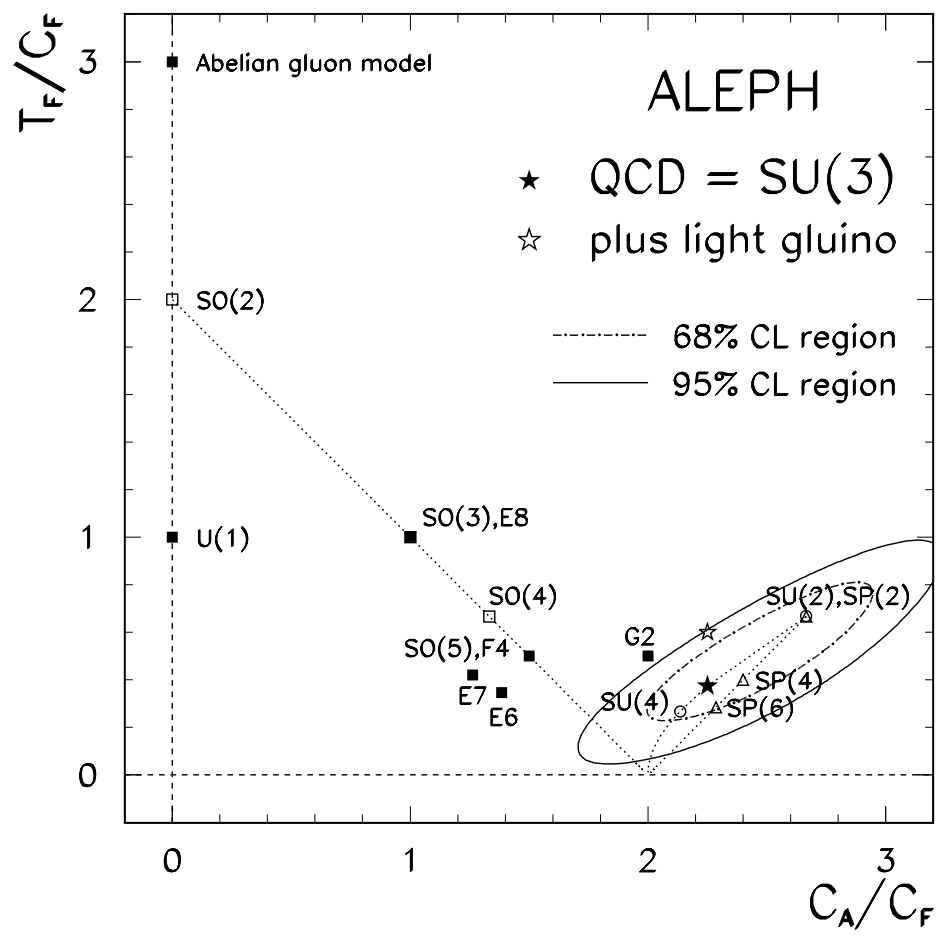

Figure 34: Combined results for the colour factor ratios compared with predictions for simple Lie groups. Also shown is the point for QCD extended by a light gluino. 


\section{Semi-Soft QCD}

In this section a number of quantities related to the so-called "semi-soft" regime of QCD are investigated. The observables considered here are sensitive to multiple gluon radiation at low energies and small emission angles. As a consequence, perturbative predictions typically contain terms corresponding to logarithms of gluon energies or emission angles which are non-negligible at every order of $\alpha_{s}$. Reliable predictions can be obtained by resumming such terms (so-called "leading", "next-to-leading" logarithms, etc.) to all orders. This is the basis of the parton shower approach introduced in Section 1.1. Various calculations, depending on what terms are included, are abbreviated as LLA, NLLA, MLLA (modified leading-log approximation), etc.

An additional common aspect of analyses considered in this section is that hadronization plays a significant role. The theoretical predictions for the observables here are thus less well understood than those for so-called "hard" processes considered in the previous section, i.e. those involving large momentum transfers. A variety of different observables are considered, each of which helps to build up a picture of the production of final state particles. For some quantities, the perturbatively predicted features are easily recognized in the final state hadrons; in others, such features are washed out by hadronization effects.

In Section 4.1, quantities are considered for which gluon interference effects are particularly important. Section 4.2 presents measurements of the multiplicity distribution of charged particles. An investigation of intermittency effects in multiplicity distributions in rapidity space is presented in Section 4.3. In Section 4.4, the internal structure of quark and gluon jets is investigated by clustering the particles within the jets into "subjets", and in Section 4.5, measurements of charged particle distributions in quark and gluon jets are presented. Section 4.6 contains an analysis of prompt photon production; this provides information on the evolution of a parton cascade since the virtual mass of a quark decreases by means of both gluon and photon radiation.

\subsection{Coherence Phenomena}

In this section measurements of particle distributions are described which are expected to be sensitive to effects of QCD coherence (gluon interference). The measured quantities include the inclusive single particle momentum distribution, the two-particle and multiplicity-multiplicity correlation, and the three-particle energy-multiplicity-multiplicity correlation. In addition, interjet coherence is studied using the particle and momentum flow between jets in three-jet events. Various explanations for the observed effects are considered.

The existence of two phases in the description of hadron production, the first calculable with perturbation theory and the second not, leads to a fundamental difficulty in investigations of coherence effects. Often it is found that a quantum mechanical effect at the parton level can be effectively parametrized in the hadronization stage. It is difficult therefore to make conclusive statements about the perturbative level. More important are the constraints placed on the hadronization mechanism using the assumption that the parton level is given by perturbative QCD. That is, one learns whether the hadronization is such that certain parton level effects are washed out or not, and, as a result, one obtains information about the nature of parton confinement. 


\subsubsection{Inclusive Distribution of $-\ln x_{p}$}

One of the simplest quantities sensitive to coherence is the single particle inclusive momentum distribution. In order to examine the low momentum region more closely, one can transform to the variable $\xi=-\ln x_{p}$, where $x_{p}=p / p_{\text {beam }}$. As discussed in Section 1.1.2, an effect of gluon interference at the parton level is the suppression of soft gluons, which is implemented in the parton-shower approach by means of angular ordering [18]. In order to relate the parton-level predictions for $D(\xi)=\left(1 / \sigma_{t o t}\right)(d \sigma / d \xi)$ to hadron-level measurements, some assumption about the hadronization stage must be made. The simplest ansatz is the hypothesis of Local PartonHadron Duality (LPHD) [86, 107]. This states that the perturbatively computed spectrum for partons $D_{Q C D}(\xi)$ should be directly proportional to the corresponding distribution for hadrons, $D_{\text {had }}(\xi)$,

$$
D_{\text {had }}(\xi)=k_{\text {had }} D_{Q C D}(\xi)
$$

where the constant $k_{\text {had }}$ must be determined by comparing the prediction to experimental data. More detailed treatments of hadronization and particle decays are provided by Monte Carlo models, such as JETSET, HERWIG and ARIADNE (see Section 2.3.1), all of which also include angular ordering of parton emissions.

The analysis presented here is based on charged particles without particle identification. The inclusive charged particle spectrum $D_{c h}(\xi)=\left(1 / \sigma_{t o t}\right)(d \sigma / d \xi)$ was measured using the same data ( 571800 events) and analysis technique as for the distributions given in Section 2 (see also Section 1.3.1). Corrections for detector effects were made using bin-by-bin correction factors as described in Section 1.3.2. Systematic uncertainties were estimated by variation of the experimental cuts, which leads to errors in the central region $(2.4<\xi<4.8)$ of the distribution of around $0.2-0.5 \%$. In addition, the model dependence of the correction factors was investigated by means of simplified correction factors as described in Section 1.3 .2 using the models JETSET, HERWIG and ARIADNE. This leads to systematic errors of $0.3-1.0 \%$ in the central region.

The measured $\xi$ distribution is given in Table 22 and is shown in Fig. 35 along with the predictions of the models JETSET version 7.4, HERWIG version 5.8 and ARIADNE version 4.08. The important parameters of these models have been tuned to ALEPH data as described in Section 2.3. The data and model predictions are seen to be in qualitatively good agreement, although the data lie significantly above the predicted values at high values of $\xi$ (low momenta).

The $\xi$ distribution can be compared directly to the perturbative QCD prediction by invoking the LPHD relation (60). At the parton level, the inclusive gluon distribution $D_{M L L A}\left(\xi ; E_{c m}, \Lambda, Q_{0}\right)$ has been computed [86] using the modified leading-log approximation (MLLA), which includes the effects of angular ordering. The MLLA prediction contains in principle three parameters: the centre of mass energy $E_{c m}$, the QCD scale parameter, $\Lambda$, and a virtuality cutoff, $Q_{0}$. The minimum parton virtuality $Q_{0}$ defines the boundary between the perturbative and non-perturbative phases. One would like $Q_{0}$ to be large enough so that the strong coupling constant $\alpha_{s}\left(Q_{0}^{2}\right)$ is small, implying $\ln \left(Q_{0} / \Lambda\right)>1$. Evaluation of $D_{M L L A}\left(\xi ; E_{c m}, \Lambda, Q_{0}\right)$ turns out to be computationally difficult except for the special case $Q_{0}=$ $\Lambda$, the so-called limiting spectrum. It has been argued [86], however, that $D_{M L L A}\left(\xi ; E_{c m}, \Lambda, Q_{0}\right)$ should change very little when $Q_{0}$ is decreased from $\Lambda \cdot e$ to $\Lambda$ (i.e. $\ln \left(Q_{0} / \Lambda\right)$ decreasing from 1 to 0$)$ so that one expects that the limiting spectrum, with which the data are compared here, gives a reasonable approximation for the parton level. 


\begin{tabular}{|c|c|}
\hline Interval & $(1 / N)(d N / d \xi) \pm$ stat. \pm sys. \\
\hline $0.200-0.300$ & $0.0447 \pm 0.0011 \pm 0.0009$ \\
$0.300-0.400$ & $0.0915 \pm 0.0017 \pm 0.0018$ \\
$0.400-0.500$ & $0.1483 \pm 0.0021 \pm 0.0028$ \\
$0.500-0.600$ & $0.2283 \pm 0.0026 \pm 0.0043$ \\
$0.600-0.700$ & $0.3320 \pm 0.0032 \pm 0.0064$ \\
$0.700-0.800$ & $0.4514 \pm 0.0037 \pm 0.0094$ \\
$0.800-0.900$ & $0.5971 \pm 0.0043 \pm 0.0128$ \\
$0.900-1.000$ & $0.7699 \pm 0.0049 \pm 0.0140$ \\
$1.000-1.100$ & $0.9605 \pm 0.0054 \pm 0.0119$ \\
$1.100-1.200$ & $1.169 \pm 0.006 \pm 0.011$ \\
$1.200-1.300$ & $1.413 \pm 0.007 \pm 0.013$ \\
$1.300-1.400$ & $1.653 \pm 0.007 \pm 0.016$ \\
$1.400-1.500$ & $1.905 \pm 0.008 \pm 0.018$ \\
$1.500-1.600$ & $2.199 \pm 0.008 \pm 0.021$ \\
$1.600-1.700$ & $2.473 \pm 0.009 \pm 0.024$ \\
$1.700-1.800$ & $2.757 \pm 0.009 \pm 0.025$ \\
$1.800-1.900$ & $3.043 \pm 0.010 \pm 0.025$ \\
$1.900-2.000$ & $3.337 \pm 0.010 \pm 0.025$ \\
$2.000-2.100$ & $3.653 \pm 0.011 \pm 0.027$ \\
$2.100-2.200$ & $3.913 \pm 0.011 \pm 0.027$ \\
$2.200-2.300$ & $4.211 \pm 0.011 \pm 0.027$ \\
$2.300-2.400$ & $4.509 \pm 0.012 \pm 0.028$ \\
$2.400-2.500$ & $4.747 \pm 0.012 \pm 0.030$ \\
$2.500-2.600$ & $4.975 \pm 0.013 \pm 0.031$ \\
$2.600-2.700$ & $5.240 \pm 0.013 \pm 0.030$ \\
$2.700-2.800$ & $5.431 \pm 0.013 \pm 0.029$ \\
\hline
\end{tabular}

\begin{tabular}{|c|c|}
\hline Interval & $(1 / N)(d N / d \xi) \pm$ stat. \pm sys. \\
\hline $2.800-2.900$ & $5.668 \pm 0.013 \pm 0.028$ \\
$2.900-3.000$ & $5.863 \pm 0.014 \pm 0.026$ \\
$3.000-3.100$ & $6.054 \pm 0.014 \pm 0.028$ \\
$3.100-3.200$ & $6.124 \pm 0.014 \pm 0.029$ \\
$3.200-3.300$ & $6.269 \pm 0.014 \pm 0.031$ \\
$3.300-3.400$ & $6.377 \pm 0.014 \pm 0.030$ \\
$3.400-3.500$ & $6.474 \pm 0.015 \pm 0.027$ \\
$3.500-3.600$ & $6.455 \pm 0.014 \pm 0.025$ \\
$3.600-3.700$ & $6.503 \pm 0.015 \pm 0.026$ \\
$3.700-3.800$ & $6.497 \pm 0.015 \pm 0.027$ \\
$3.800-3.900$ & $6.436 \pm 0.015 \pm 0.027$ \\
$3.900-4.000$ & $6.338 \pm 0.014 \pm 0.029$ \\
$4.000-4.100$ & $6.149 \pm 0.014 \pm 0.033$ \\
$4.100-4.200$ & $6.062 \pm 0.014 \pm 0.034$ \\
$4.200-4.300$ & $5.899 \pm 0.014 \pm 0.033$ \\
$4.300-4.400$ & $5.698 \pm 0.014 \pm 0.033$ \\
$4.400-4.500$ & $5.554 \pm 0.014 \pm 0.034$ \\
$4.500-4.600$ & $5.127 \pm 0.013 \pm 0.038$ \\
$4.600-4.700$ & $4.778 \pm 0.013 \pm 0.045$ \\
$4.700-4.800$ & $4.641 \pm 0.013 \pm 0.051$ \\
$4.800-4.900$ & $4.190 \pm 0.012 \pm 0.055$ \\
$4.900-5.000$ & $3.772 \pm 0.012 \pm 0.061$ \\
$5.000-5.100$ & $3.358 \pm 0.011 \pm 0.046$ \\
$5.100-5.200$ & $2.951 \pm 0.011 \pm 0.067$ \\
$5.200-5.300$ & $2.565 \pm 0.010 \pm 0.227$ \\
$5.300-5.400$ & $2.185 \pm 0.010 \pm 0.690$ \\
\hline
\end{tabular}

Table 22: Distribution of $\xi=-\ln x_{p}$.

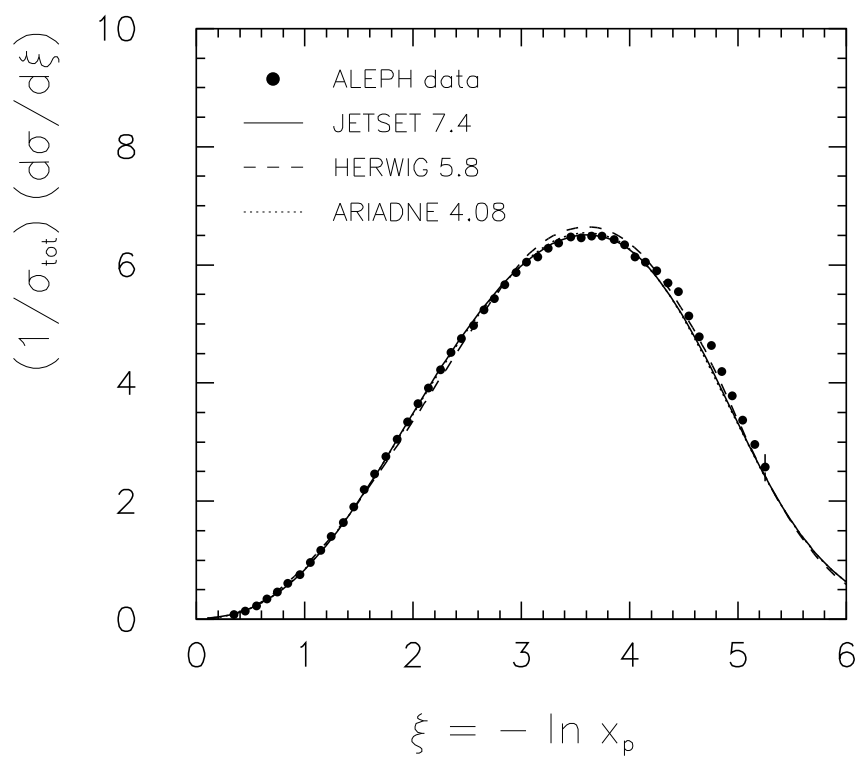

Figure 35: The measured charged particle inclusive distribution of $\xi=$ $-\ln x_{p}$ and predictions of Monte Carlo models. 
Figure 36 shows the measured $\xi$ distribution compared to the prediction of MLLA and LPHD, Eq. (60). The MLLA formula $D_{M L L A}(\xi)$ is expected to be valid only in the region around the peak of the distribution. Using the range $2.6 \leq \xi \leq 4.5$ results in $k_{c h}=0.874$ and $\Lambda=0.266 \mathrm{GeV}$, with negligibly small fit errors but a very bad $\chi^{2}$ of 970 for 17 degrees of freedom. More important than the parameter values themselves is the observation of how well the MLLA formula agrees with the data. Although the MLLA distribution is qualitatively the same shape as the measurement in the region near the peak, it is significantly narrower.



Figure 36: The charged particle inclusive distribution of $\xi=-\ln x_{p}$ and predictions of analytical QCD calculations, assuming local partonhadron duality.

The parton momentum distribution has also been calculated in ref. [108] by computing the higher moment corrections to a Gaussian form. This can be used with the LPHD hypothesis (60) to relate the parton to charged hadron spectra. The inclusive spectrum is then

$$
D_{c h}(\xi)=\frac{n_{c h}}{\sigma \sqrt{2 \pi}} \exp \left[\frac{1}{8} k-\frac{1}{2} s \delta-\frac{1}{4}(2+k) \delta^{2}+\frac{1}{6} s \delta^{3}+\frac{1}{24} k \delta^{4}\right]
$$

where $n_{c h}$ gives the overall normalization, $\delta=(\xi-\bar{\xi}) / \sigma$, and the mean, $\bar{\xi}$, width, $\sigma$, skewness, $s$, and kurtosis, $k$, have been computed by Fong and Webber [108] as a function of an effective QCD scale parameter $\Lambda$. Higher order effects are expected to give an (energy independent) additive correction of $O(1)$ for the mean, and smaller (asymptotically vanishing) corrections for $\sigma, s$ and $k$. In this analysis $\Lambda, n_{c h}$ and the $O(1)$ correction are fitted, and the other higher order corrections are set to zero. Using the same fit region as above, $2.6 \leq \xi \leq 4.5$, results in $\Lambda=0.139 \pm 0.001 \mathrm{GeV}, n_{c h}=22.80 \pm 0.02$ and the additive correction to $\bar{\xi}$, $O(1)=-1.350 \pm 0.007$ with a $\chi^{2}$ of 128 for 17 degrees of freedom.

The discrepancies observed between the parton-level QCD formulae and the data can be interpreted as a limit on the combined effects of perturbative higher orders, hadronization and subsequent decays. The difference between the two QCD curves in Fig. 36 gives a measure of the uncertainty at the perturbative level; this is seen to be not negligible, even in the peak region. The effects of hadronization and decays as well are seen to be large, as indicated by the better description of the data by the Monte Carlo models in Fig. 35. 


\subsubsection{Energy Dependence of the Peak of the $-\ln x_{p}$ Distribution}

One of the primary interests in the $\xi$ distribution lies in the energy dependence of its peak position $\xi^{*}$. This can be determined by fitting a parametrization of the distribution in the peak region. Possible parametrizations include a Gaussian and the distorted Gaussian of Eq. (61). In order to investigate the energy dependence, $\xi^{*}$ is determined using the distribution at $E_{c m}=91.2 \mathrm{GeV}$ presented in Section 4.1.1, as well as with the corresponding distributions from the TASSO experiment at $E_{c m}=14,22,35$ and $44 \mathrm{GeV}$ [80], and from ALEPH at $E_{c m}=133 \mathrm{GeV}[109]$. The fit regions were chosen to use the points having distribution values of at least $70 \%$ of the highest value (see Table 23 ). The $\xi^{*}$ values were determined using the distorted Gaussian (61), where the normalization parameter $n_{c h}$, the effective QCD scale $\Lambda$, and the $O(1)$ correction are fitted, and the other higher order corrections were set to zero.

In general the experimental systematic errors are correlated between bins, and this must be taken into account when determining $\xi^{*}$. This was done by assuming the following model for the covariance matrix,

$$
V_{i j}=\delta_{i j} \sigma_{i s t a t}^{2}+\sigma_{i s y s} \sigma_{j s y s}\left(1-2 \frac{\left|\xi_{i}-\xi_{j}\right|}{\Delta \xi_{\max }}\right),
$$

where $\xi_{i}$ and $\xi_{j}$ are the $\xi$ values in the centres of bins $i$ and $j$, and $\Delta \xi_{\max }$ is the difference in $\xi$ between the centres of the first and last bins in the fit region. This assumption for the systematic part of the covariance matrix means that points on opposite ends of the fit range have a correlation coefficient $\rho=-1$, points separated by half the fit range have $\rho=0$, and of course $\rho=1$ for $i=j$. Although there is no particular reason to believe that this model is correct, it is the most conservative choice for purposes of determining the error in the peak position, since the negative correlation for widely separately points corresponds to a shift of the peak.

The experimental systematic errors for the distribution at $91.2 \mathrm{GeV}$ were determined as described in Section 1.3.2 (see also Section 4.1.1). For the distributions from the TASSO experiment, only total errors are given in ref. [80]. The statistical and systematic components of these errors were deduced from the number of events recorded at each centre of mass energy. The covariance matrix (62) was used for the TASSO distributions ( $E_{c m} \leq 44 \mathrm{GeV}$ ) and for the distribution from ALEPH at $91.2 \mathrm{GeV}$. For the analysis at $E_{c m}=133 \mathrm{GeV}$, the determination of the experimental systematic error of $\xi^{*}$ is described in ref. [109]. The resulting $\xi^{*}$ values are shown in Table 23.

The systematic uncertainty in $\xi^{*}$ due to the choice of the parametrization of the distribution was investigated by repeating the fits with a Gaussian function at each of the centre-of-mass energies. The differences between the results with the distorted and the usual Gaussians have a mean of 0.064 and a standard deviation of 0.011 . Thus to a good approximation, the effect of changing the fit function is a common shift at all energies. A Monte Carlo study based on $50 \mathrm{k}$ events generated by the JETSET model at $E_{c m}=14,22,35,44,91.2$ and $133 \mathrm{GeV}$ showed the same behaviour, with almost exactly the same mean shift and standard deviation.

In the QCD prediction for $\xi^{*}$ as a function of $E_{c m}$ examined below, a constant shift in $\xi^{*}$ can be absorbed into the effective QCD scale parameter, and thus positively correlated errors do not play a role in the test of the prediction. In order for the fit-function errors to be highly correlated, it was found that the fit ranges must be chosen in a consistent way at each energy, i.e. by using the full width at a fixed fraction $f$ of the maximum distribution value. Because of large data sample at $E_{c m}=91.2 \mathrm{GeV}$, a small fit range would be possible, and this would result 


\begin{tabular}{|c|c|c|c|c|}
\hline$E_{c m}(\mathrm{GeV})$ & fit range & $\xi^{*} \pm \Delta \xi_{e x p}^{*}$ & points fitted & $\chi^{2} /$ dof \\
\hline 14 & {$[1.6,3.2]$} & $2.453 \pm 0.053$ & 8 & 0.27 \\
22 & {$[2.0,3.4]$} & $2.738 \pm 0.057$ & 7 & 1.52 \\
35 & {$[2.0,3.8]$} & $3.072 \pm 0.023$ & 9 & 0.91 \\
44 & {$[2.0,4.0]$} & $3.174 \pm 0.040$ & 10 & 0.62 \\
91.2 & {$[2.4,4.8]$} & $3.670 \pm 0.009$ & 24 & 9.04 \\
133 & {$[2.6,5.2]$} & $3.923 \pm 0.081$ & 13 & 1.31 \\
\hline
\end{tabular}

Table 23: Fit results for $\xi^{*}$ based on a distorted Gaussian. The fit ranges correspond to the full widths at 0.7 of the maximum distribution value. The experimental error includes both the statistical and experimental systematic uncertainties. The full error matrix for the measurements is given by Eq. (63) (see text).

in a smaller uncertainty due to the choice of fit function. Owing to the smaller data samples at the other energies, however, it was necessary to use a larger fit range, and as a compromise, the fraction $f=0.7$ was taken for all energies.

The sensitivity of the results to the fit range was investigated by repeating the analysis with $f=0.5$ and $f=0.9$. At $E_{c m}=91.2 \mathrm{GeV}$, this resulted in shifts of $\xi^{*}$ of +0.015 and -0.028 , respectively. At the other centre of mass energies, the changes in $\xi^{*}$ were largely consistent with those expected from statistical fluctuations. A Monte Carlo study with 50k events at each energy, however, showed variations in $\xi^{*}$ when using $f=0.9$ which could not be easily interpreted as a common shift. The rms difference of 0.023 between the two cases $f=0.7$ and $f=0.9$ was thus assigned as an additional uncorrelated uncertainty due to the choice of the fit range.

The covariance matrix for energy points $i$ and $j$ was thus taken to be

$$
V_{i j}=\left(\Delta \xi^{* 2}+(0.025)^{2}\right) \delta_{i j}+(0.064)^{2},
$$

where $\Delta \xi^{*}$ is the quadratic sum of statistical and (experimental) systematic errors, $(0.025)^{2}=$ $(0.011)^{2}+(0.023)^{2}$ is the uncorrelated error due to the fit function and range, respectively, and 0.064 is the correlated error due to choice of the fit function.

Figure 37 shows the fitted $\xi^{*}$ values as a function of centre of mass energy. Using the modified leading-log approximation (MLLA), this is predicted to be [86]

$$
\xi^{*}=Y\left(\frac{1}{2}+a \sqrt{\frac{\alpha_{s}(Y)}{32 N_{c} \pi}}-a^{2} \frac{\alpha_{s}(Y)}{32 N_{c} \pi}+\cdots\right)
$$

where

$$
\begin{array}{ll}
Y=\ln E_{j e t} / \Lambda, & E_{j e t}=E_{c m} / 2, \quad \alpha_{s}(Y)=\frac{1}{2 b_{0} Y}, \\
a=\frac{11}{3} N_{c}+\frac{2 n_{f}}{3 N_{c}^{2}}, & b_{0}=\frac{11 N_{c}-2 n_{f}}{12 \pi} .
\end{array}
$$

The number of colours $N_{c}$ as well as the number of light quark flavours $n_{f}$ is taken here to be three (as in ref. [86]). This leaves only one adjustable parameter, the QCD scale $\Lambda$. Because of uncertainties from higher order corrections, it is not possible to directly identify $\Lambda$ in the MLLA calculation with $\Lambda_{\overline{M S}}$, and it must be treated as a phenomenological parameter. The 
two quantities are nevertheless expected to be of approximately the same magnitude. The first term in (64), $\frac{1}{2} Y$, is the leading order prediction (Double Logarithmic Approximation, DLA).

In the formula (64) for $\xi^{*}$ versus $E_{c m}$, a constant shift in $\xi^{*}$ can be absorbed (to leading order) into the effective QCD scale parameter $\Lambda$. Therefore the correlated uncertainty of 0.064 is not important in the comparison of the data with the QCD curves, and only the uncorrelated components of the errors are shown. The MLLA curve gives $\chi^{2}=1.3$ and the DLA curve $\chi^{2}=31.0$ for 5 degrees of freedom.

Equation (64) takes no account of quark flavour or mass effects. Monte Carlo studies show, however, that the $\xi^{*}$ values are sensitive to the event flavour, and furthermore the mixture of flavours varies as a function of the centre-of-mass energy. By comparing the $\xi^{*}$ values predicted for the Standard Model mixture of flavours to that of light quarks only, a correction factor for the flavour dependence can be derived and applied to Eq. (64) as $\xi_{c o r r}^{*}=\xi^{*} \cdot C\left(E_{c m}\right)$. This was done by determining the ratio $C=\xi^{*}$ (all flavours $) / \xi^{*}(u \bar{u}$ only) using the JETSET model at several points in the energy range $12 \mathrm{GeV}<E_{c m}<180 \mathrm{GeV}$, which was then parametrized by a function $C\left(E_{c m}\right)=a+b \exp \left(-E_{c m} / c\right)$, with $a=0.976, b=0.0612$, and $c=31.3 \mathrm{GeV}$. This correction decreases from 1.02 to 0.99 in the energy region between 14 and $44 \mathrm{GeV}$, and is almost constant for $E_{c m}>44 \mathrm{GeV}$.

When this is applied to the QCD predictions, the quality of the fit becomes somewhat worse, with $\chi^{2}=62.0$ for DLA and $\chi^{2}=10.6$ for MLLA for five degrees of freedom. The general picture remains that the higher order effects included in the MLLA improve the description of the data. The very good $\chi^{2}$ value from the MLLA without flavour correction, however, must be partially accidental. The fitted curves including the flavour correction are shown in Fig. 37. Fitted curves without the flavour correction can be found in [109].

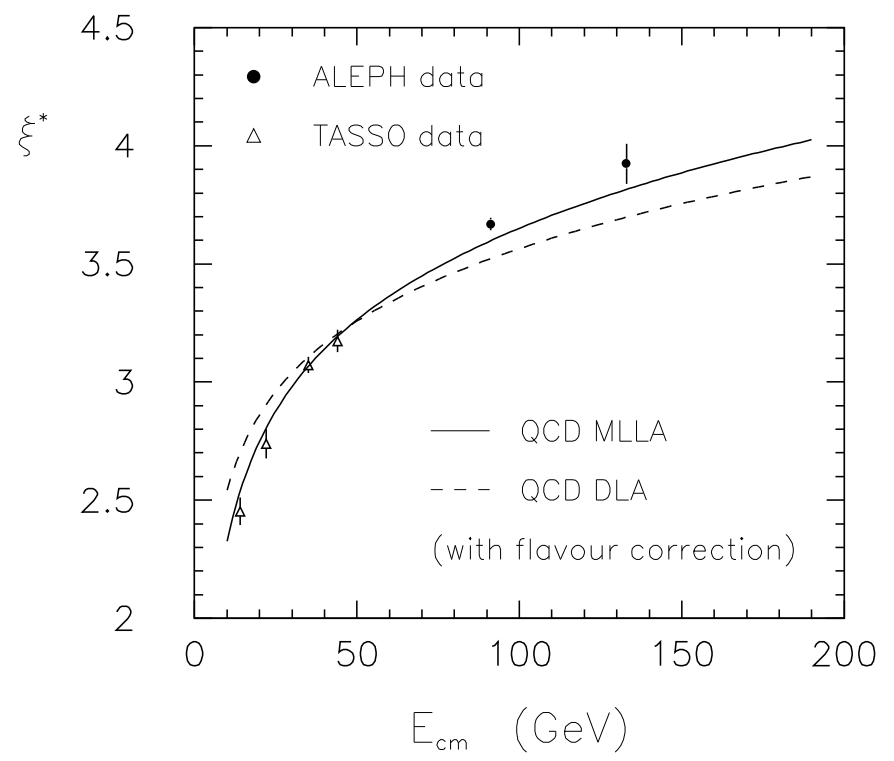

Figure 37: The peak position of the $-\ln x_{p}$ distribution, $\xi^{*}$, as a function of the centre of mass energy, and the leading order (DLA) and next-to-leading order (MLLA) QCD predictions including a correction for the energy dependence of the quark flavour mixture (see text). In addition to the error bars shown, there is a correlated uncertainty of 0.064 due to the choice of fit function.

In determining the $\xi^{*}$ values in Table 23, the fit ranges were all determined by the full width at $f=0.7$ of the maximum distribution value. This procedure was necessary in order to have highly correlated errors due to the choice of fit function, and led to a relatively large fit range at $91.2 \mathrm{GeV}(2.4 \leq \xi \leq 4.8)$. In order to compare with measurements from other experiments, however, one can reduce the fit range at $91.2 \mathrm{GeV}$ to give a smaller total error. Using e.g. the full width at a fraction $f=0.9$ of the maximum gives $2.9<\xi<4.3$. This results in $\xi^{*}=3.642$ with the distorted Gaussian and 3.610 with a Gaussian, i.e. a difference of 0.032 . Taking the distorted Gaussian for the best value, the difference with respect to the Gaussian 
for the fit-function error, and the difference of 0.028 between fits using $f=0.7$ and $f=0.9$ for the fit-range error, one obtains

$$
\begin{aligned}
\xi^{*}(91.2 \mathrm{GeV}) & =3.642 \pm 0.017(\text { exp. }) \pm 0.032 \text { (fit function) } \pm 0.028 \text { (fit range) } \\
& =3.642 \pm 0.046 .
\end{aligned}
$$

This result is in agreement with the values reported by OPAL [110] of $\xi^{*}=3.603 \pm$ 0.013 (stat.) \pm 0.040 (sys.), and by L3 [111], $\xi^{*}=3.71 \pm 0.01$ (stat.) \pm 0.05 (sys.).

\subsubsection{Particle-Particle Correlations}

As first pointed out in [112], coherence effects related to angular ordering can be investigated with the particle-particle correlation function, PPC. This is defined by considering two elements of solid angle $d \vec{\Omega}_{a}$ and $d \vec{\Omega}_{b}$, with an opening angle $\chi=\cos ^{-1}\left(\vec{\Omega}_{a} \cdot \vec{\Omega}_{b}\right)$. The function $\operatorname{PPC}(\chi)$ is constructed as a measure of the probability to find a particle in both $d \vec{\Omega}_{a}$ and $d \vec{\Omega}_{b}$. In terms of the two-particle inclusive cross section, it is defined as

$$
\operatorname{PPC}(\chi)=\iint \frac{1}{\sigma} \frac{d \sigma\left(e^{+} e^{-} \rightarrow a+b+X\right)}{d \vec{\Omega}_{a} d \vec{\Omega}_{b}} \delta\left(\cos ^{-1}\left(\vec{\Omega}_{a} \cdot \vec{\Omega}_{b}\right)-\chi\right) d \vec{\Omega}_{a} d \vec{\Omega}_{b},
$$

which can be determined experimentally using charged particles as

$$
\langle\operatorname{PPC}(\chi)\rangle_{\text {bin } k}=\frac{1}{N_{\text {events }}} \sum_{\text {events }} \sum_{i, j=1}^{N_{c h}} \frac{1}{N_{c h}^{2}} \frac{1}{\Delta \chi} \int_{b i n k} \delta\left(\chi_{i j}-\chi^{\prime}\right) d \chi^{\prime} .
$$

Here $\chi_{i j}$ is the angle between the particles $i$ and $j$, the sum over $i$ and $j$ is made over all pairs of $N_{c h}$ charged particles in an event, and is then averaged over all events. The quantity $\mathrm{PPC}(\chi)$ is similar in construction to the energy-energy correlation EEC described in Section 3.2 , with the difference that each pair of particles is weighted by $w_{i j}=1 / N_{c h}^{2}$, rather than by the product of the particles' scaled energies, $w_{i j}=E_{i} E_{j} / E_{c m}^{2}$. Also in analogy with the EEC, the particle-particle correlation asymmetry, $\operatorname{PPCA}(\chi)$, can be constructed as

$$
\operatorname{PPCA}(\chi)=\operatorname{PPC}(\pi-\chi)-\operatorname{PPC}(\chi)
$$

A measurement of the PPCA based on charged particles was carried out with approximately 800000 hadronic events recorded by the ALEPH detector in 1992-93. The hadronic event selection and correction procedure for detector effects were carried out as described in Sections 1.3.1 and 1.3.2, respectively. Systematic uncertainties were estimated by variation of the track and event selection criteria. In addition, the model dependence of the correction factors was estimated by constructing simplified corrections with several models, as described in Section 1.3.2.

Figure 38(a) shows the measured PPCA as a function of the angle $\chi$ along with the predictions of several parton-shower based Monte Carlo models. The models are in good agreement with the data, with the exception of NLLjet, for which a discrepancy is seen in the region $10^{\circ}<\chi<40^{\circ}$. Figure 38(b) shows the data compared with the same models, with the difference that the angular ordering in the parton shower has not been included. (For HERWIG and ARIADNE this option is not possible.) The parameters of the models were 

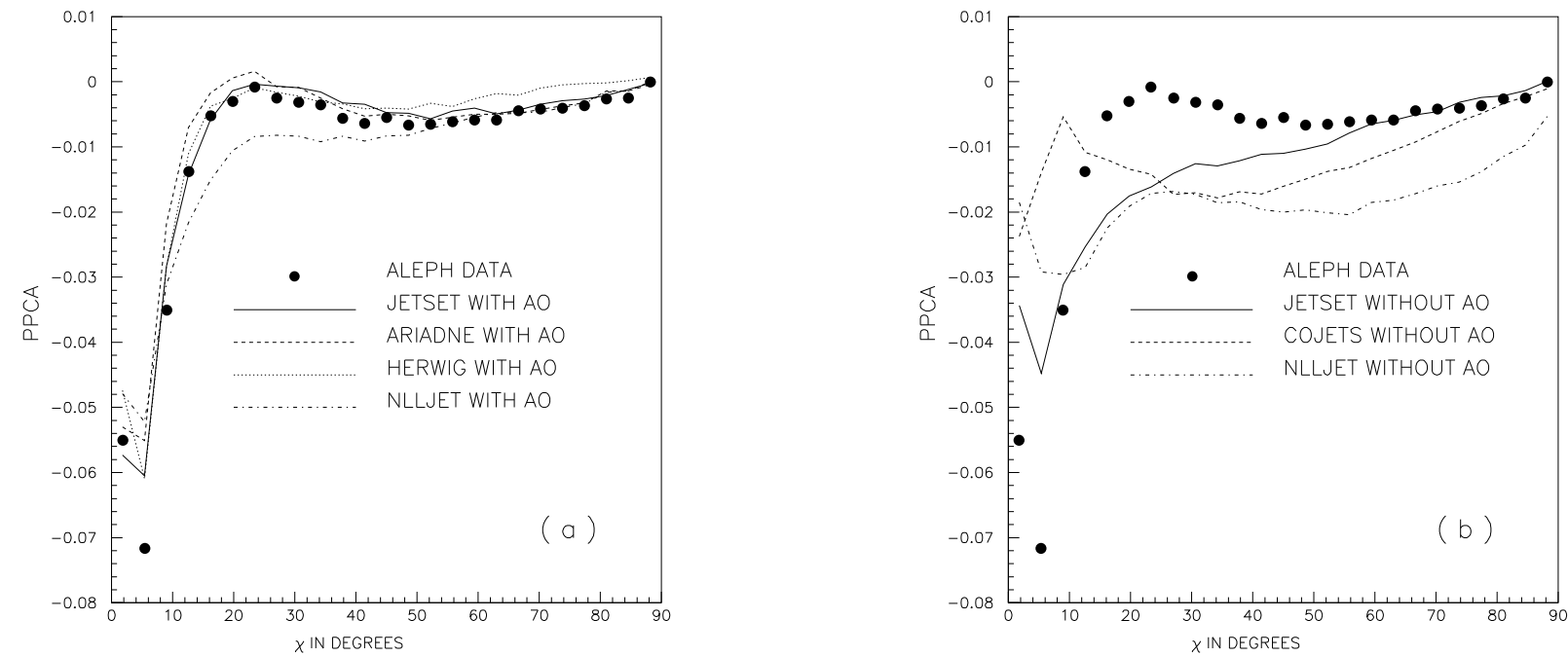

Figure 38: The particle-particle correlation asymmetry PPCA as measured by ALEPH and as predicted by various parton-shower based Monte Carlo models (a) with and (b) without angular ordering. The errors are highly correlated from point to point and are not shown on the plot. The errors including model uncertainties are indicated by the bands in Fig. 39.
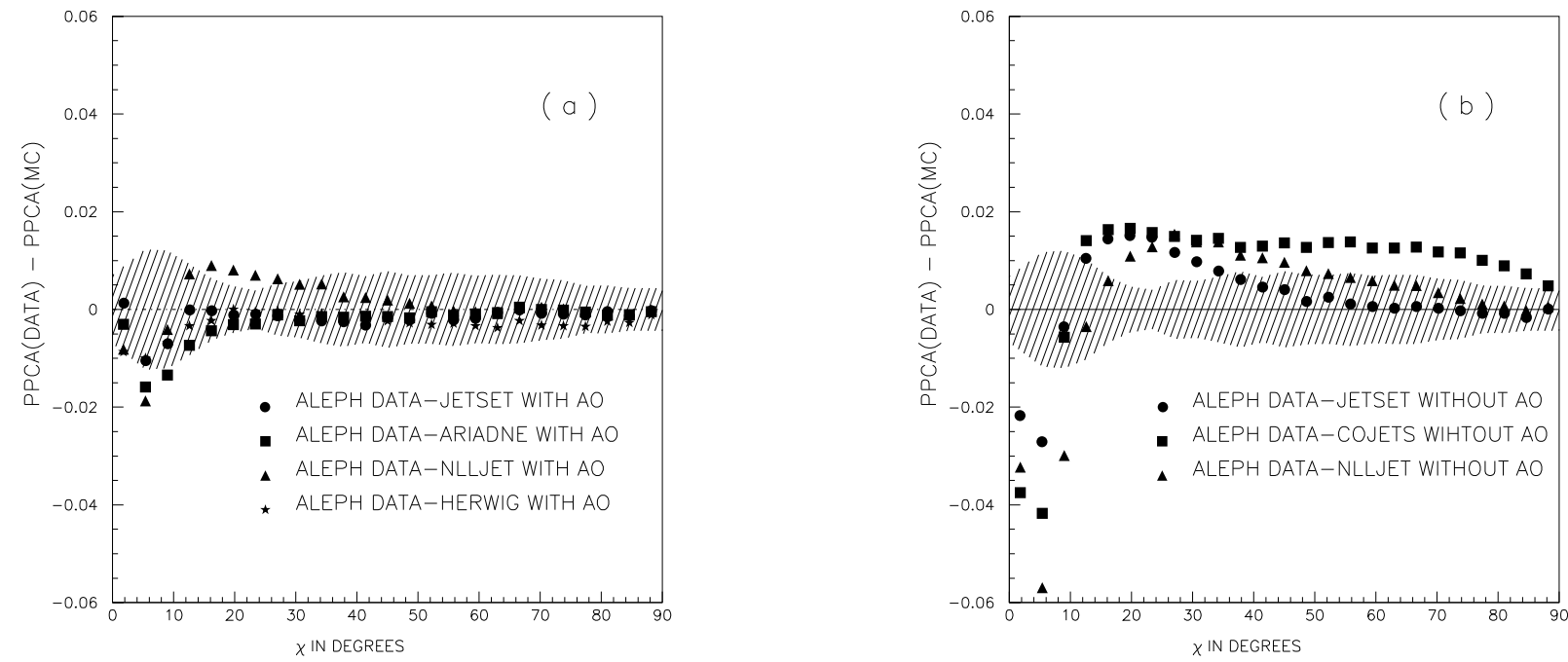

Figure 39: The difference of measured minus predicted PPCA for several parton-shower based Monte Carlo models (a) with and (b) without angular ordering. The shaded band represents \pm 1 standard deviation for statistical and systematic errors added in quadrature. 
tuned to data, as described in Section 2.3, including separate tuning for the models without angular ordering. (For COJETS [113], the parameters were left at their default values, derived by the model's authors from comparison to a variety of LEP and lower energy data.)

The models without angular ordering are in significant disagreement with the data, indicating that the PPCA is sensitive to coherence effects. The significance of the discrepancy is made clear in Fig. 39 which shows the difference of the measured and predicted PPCA values for models with and without angular ordering. The shaded band indicates \pm 1 standard deviation from statistical and systematic errors added in quadrature. Here the systematic error includes uncertainties both in the data as well as in the model prediction. The latter were estimated by individually varying the model parameters $\pm 1 \sigma$ from their fitted values. For JETSET, in addition, alternative implementations of the angular ordering procedure as well as Bose-Einstein correlations were tested, although these were found to not have any significant effect on the PPCA.

\begin{tabular}{|l|l|r|}
\hline Model & PPCA & EMMC \\
\hline & \multicolumn{2}{|c|}{$\chi^{2}$} \\
\hline JETSET AO & 2.3 & 31.9 \\
\hline ARIADNE AO & 6.1 & 38.5 \\
\hline HERWIG AO & 5.2 & 30.9 \\
\hline NLLjet AO & 11.4 & 127.0 \\
\hline \hline JETSET NOAO & 40.4 & 93.5 \\
\hline COJETS NOAO & 102.0 & 333.2 \\
\hline NLLjet NOAO & 70.1 & 326.5 \\
\hline
\end{tabular}

Table 24: The $\chi^{2}$ values from a comparison of measured and predicted particle-particle correlation asymmetry PPCA (24 degrees of freedom) and energy-multiplicity-multiplicity correlation function $C(\varphi)$ (49 degrees of freedom). Models were used both with angular ordering (AO) and without (NOAO).

Although the discrepancy of NLLjet including angular ordering is not entirely understood, there is a clear tendency that angular ordering greatly improves the description of the data. The $\chi^{2}$ values from comparing each model with data are given in Table 24 . Bin-to-bin correlations were estimated by generating 500 Monte Carlo samples of 2000 events each. The $\chi^{2}$ values alone are difficult to interpret since conservative estimates of systematic errors result in very low values of $\chi^{2}$. Used as a basis for comparing models, however, they show a clear preference for those with angular ordering. The results presented here are in qualitative agreement with those of [114].

\subsubsection{Energy-Multiplicity-Multiplicity Correlations}

The energy-energy-multiplicity correlation function (EMMC) was first proposed in order to investigate the azimuthal correlation of two soft particles emitted at a similar polar angle with respect to the event axis [115]. The EMMC is defined as

$$
\begin{aligned}
& C_{E M M}\left(\varphi, \eta_{\min }, \eta_{\max }\right)= \\
& \quad \frac{1}{\sigma_{\text {tot }}} \int E_{i} d E_{i} d E_{j} d E_{k} \int_{\eta_{\min }}^{\eta_{\max }} d \eta_{j} d \eta_{k} \int_{0}^{2 \pi} d \varphi_{j} d \varphi_{k} \delta\left(\varphi-\varphi_{j}+\varphi_{k}\right) \frac{d \sigma}{d E_{i} d E_{j} d E_{k} d \eta_{j} d \eta_{k} d \varphi_{j} d \varphi_{k}}
\end{aligned}
$$


Experimentally this is constructed by considering each set of three particles in an event: $i, j, k$. Particles $j$ and $k$ have a certain polar angle $\theta$ (or pseudorapidity, $\eta=-\ln \tan \frac{1}{2} \theta$ ) and a certain azimuthal angle $\varphi=\left|\varphi_{j}-\varphi_{k}\right|$ with respect to the particle $i$. If both particles $j$ and $k$ are contained within a certain region of pseudorapidity $\eta_{\min } \leq \eta_{j, k} \leq \eta_{\max }$ then an entry is made in a histogram of $\varphi$ weighted by the energy of particle $i, E_{i}$. The weighting by $E_{i}$ tends to associate particle $i$ with the jet axis, and $\eta_{\min }$ and $\eta_{\max }$ can be chosen so that particles $j$ and $k$ correspond to soft gluon radiation. To obtain a quantity related to the correlation in azimuthal angle of particles $j$ and $k$, one normalizes to the two-particle energy-multiplicity correlation squared divided by the total energy flow:

$$
C(\varphi)=\frac{C_{E M M}\left(\eta_{\min }, \eta_{\max }, \varphi\right) C_{E}}{\left|C_{E M}\left(\eta_{\min }, \eta_{\max }\right)\right|^{2}}
$$

where

$$
C_{E}=\frac{1}{\sigma_{t o t}} \int E_{i} d E_{i} \frac{d \sigma}{d E_{i}}
$$

and

$$
C_{E M}\left(\eta_{\min }, \eta_{\max }\right)=\frac{1}{\sigma_{\text {tot }}} \int E_{i} d E_{i} d E_{j} \int_{\eta_{\min }}^{\eta_{\max }} d \eta_{j} \int_{0}^{2 \pi} d \varphi_{j} \frac{d \sigma}{d E_{i} d E_{j} d \eta_{j} d \varphi_{j}}
$$

The function $C(\varphi)$ is thus a measure of the probability to find two particles at a similar polar angle (i.e. between $\eta_{\min }$ and $\eta_{\max }$ ) separated by an azimuthal angle $\varphi$. In this analysis, the values $\eta_{\min }=1$ and $\eta_{\max }=2$ were chosen, corresponding to $15.4^{\circ} \leq \theta \leq 40.4^{\circ}$.

The function $C(\varphi)$ was measured using the same data sample, event selection criteria, and correction procedure for detector effects, as for the PPCA (Section 4.1.3). The systematic uncertainties were estimated by varying the selection cuts and by using simplified correction factors based on different Monte Carlo models, as for the PPCA. The measured function is shown in Fig. 40 along with the predictions of various Monte Carlo models, both with and without angular ordering. $\chi^{2}$ values from comparisons with various models are given in Table 24. As in the case of the PPCA, models that include angular ordering are clearly preferred.

The function $C(\varphi)$ can be computed analytically in perturbative QCD for the case of emission of two soft gluons from a quark-antiquark "colour-antenna" [115]. Interference effects lead to a suppression in the region $\varphi \sim \pi$. To leading order the correlation function $C(\varphi=\pi)$ for an infinitesimal pseudorapidity interval is $7 / 16$. The next-to-leading order correction is large, however, giving $C(\varphi)=0.93$ [116]. The measured value lies between the two, being $C(\pi)=0.78 \pm 0.02$. The results presented here are in agreement with previous studies of the EMMC [117].

\subsubsection{Particle Flow in Interjet Regions (String Effect)}

Soft gluon radiation in the regions between jets can be best described as coherent emission from all of the colour charges initially produced in the hard process. In a three-jet configuration which starts as a $q \bar{q} g$ system, perturbative QCD predicts a suppression of the soft gluon radiation between the quark and antiquark jets compared to that between the quark and gluon jets [86]. The corresponding suppression of particle flow can also be interpreted as a non-perturbative effect resulting e.g. from a boosted string, stretched from quark to gluon to antiquark, and is 

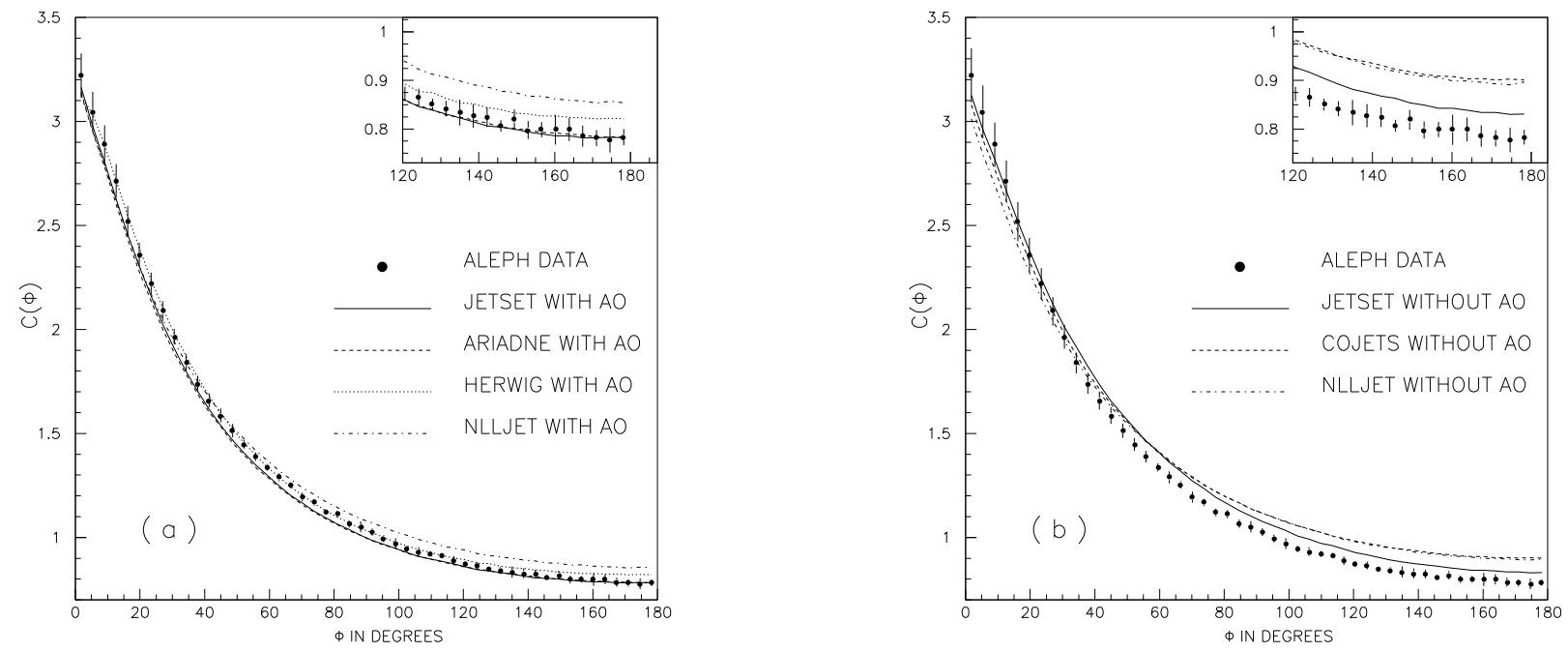

Figure 40: The energy-multiplicity-multiplicity correlation function $C(\varphi)$ as measured by ALEPH and as predicted by various parton-shower based Monte Carlo models (a) with and (b) without angular ordering.

hence known as the string effect. This effect was first observed experimentally by the JADE collaboration [118] and later by other experiments at PETRA, PEP and LEP [119].

In this analysis the string effect has been investigated by measuring the particle and momentum flow in the interjet regions of three-jet events. Approximately 112000 threejet events were selected using the Durham clustering algorithm (see Section 2.1) with a jet resolution parameter of $y_{c u t}=0.009$. Both charged and neutral particles (energy-flow objects as described in Section 1.2) were used in the analysis. In addition to the standard event selection cuts described in Section 1.3.1, the angle between the normal vector to the event plane and the beam direction was required to be less than $60^{\circ}$ in order to ensure good particle acceptance in the interjet regions.

The three jets were ordered according to their energies, $E_{1}>E_{2}>E_{3}$, estimated from the jet directions (projected onto the event plane) assuming energy and momentum conservation for massless jets. QCD based Monte Carlo models such as JETSET predict that the lowest energy jet is most frequently the gluon jet (70\%) and the highest energy jet is most often a quark (or antiquark) jet (94\%). The angle in the event plane $\varphi$ is defined to be zero for the highest energy jet (jet 1 ) as shown in Fig. 41. The reduced angle $\varphi^{\prime}$ of a particle between jets $i$ and $k$ is defined as

$$
\varphi^{\prime}=\frac{\varphi-\Phi_{i}}{\Phi_{k}-\Phi_{i}}
$$

where $\Phi_{i}$ and $\Phi_{k}$ are the jet angles. Interjet regions are defined as covering the central $40 \%$ of the reduced angle between jets $\left(0.3<\varphi^{\prime}<0.7\right)$.

The particle distribution as a function of $\varphi^{\prime}$ (without detector corrections) is shown in Fig. 42. Also shown is the prediction of the JETSET model including simulation of detector effects; this is seen to describe the data quite well.

After correction for detector effects according to the bin-by-bin procedure described in Section 1.3.2, the ratio of particle yields in the region between jets 1 and $3, N_{1,3}$, (primarily 




$R:=N_{1,3} / N_{1,2} \quad\left(E_{1}>E_{2}>E_{3}\right)$

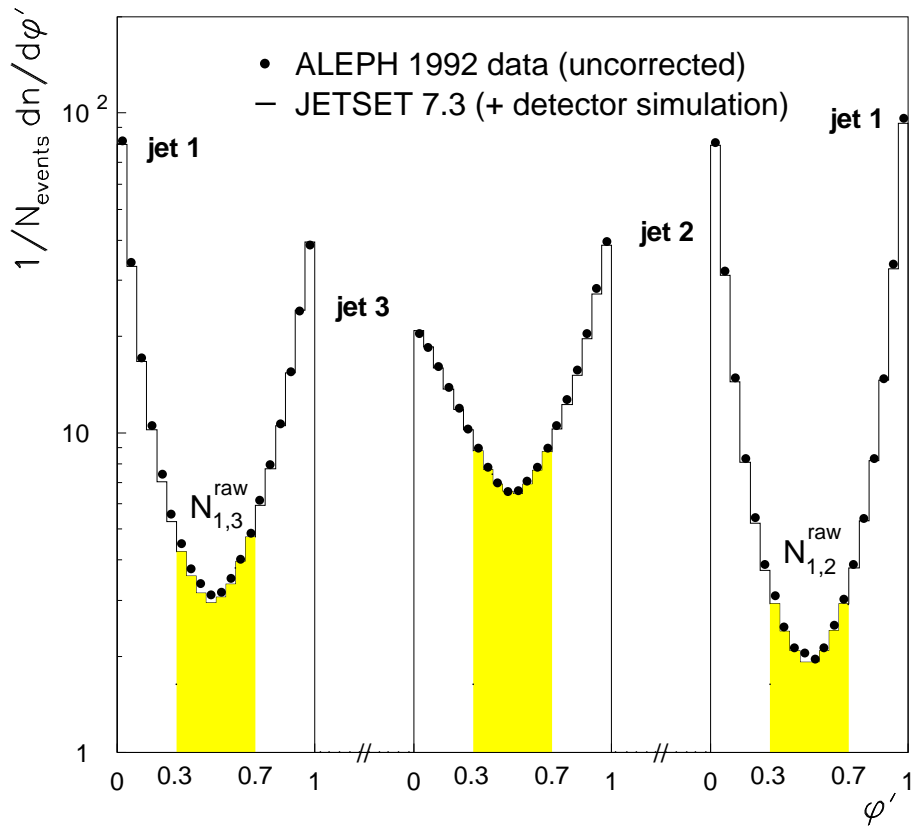

Figure 41: Definition of the interjet regions used to determine the ratio $R=$ $N_{1,3} / N_{1,2}$, which measures the strength of the string effect.

Figure 42: The inclusive particle rate $\left(1 / N_{\text {events }}\right)\left(d n / d \varphi^{\prime}\right)$ as a function of the reduced interjet angle $\varphi^{\prime}$. 
between quark and gluon jets) to $N_{1,2}$ (primarily between quark and antiquark jets) is found to be

$$
R=\frac{N_{1,3}}{N_{1,2}}=1.384 \pm 0.007 \text { (stat.) } \pm 0.035 \text { (sys.) }
$$

The analogous quantity based not on particle number but on momentum flow is found to be

$$
R_{p}=\frac{\left(\sum|p|\right)_{1,3}}{\left(\sum|p|\right)_{1,2}}=1.731 \pm 0.012 \text { (stat.) } \pm 0.029 \text { (sys.) }
$$

The systematic errors were estimated by varying track and event selection cuts. In addition, the model dependence of the detector correction factors was estimated by computing simplified corrections with different event generators as described in Section 1.3.2. The measured ratios are significantly greater than unity, indicating enhanced particle production in the angular region between the quark and gluon jets. The effect is found to increase with the momentum of the particles, as seen from the fact that $R_{p}>R$.

In Fig. 43 the measured values of $R$ and $R_{p}$ (shown as bands to indicate the total error) are compared with the predictions of several Monte Carlo models. These include several variants of JETSET 7.3 as well as HERWIG 5.6, ARIADNE 4.3 and COJETS 6.23 [113]. The most important parameters of the models have been tuned to describe global event shape and charged particle inclusive distributions (see [26]), except for COJETS (cf. Section 4.1.3). JETSET "incoherent" does not include angular ordering in the parton shower, and "azimuthal interference" includes anisotropic gluon splitting. The "closed string" model is a toy model in which the parton shower is assumed to start from a gluon-gluon state instead of a $q \bar{q}$ state. In a three-jet configuration there is thus a string spanned between each pair of jets. The JETSET model with $O\left(\alpha_{s}^{2}\right)$ matrix elements was also investigated, both with string and independent fragmentation.

The JETSET model with string fragmentation, both with the $O\left(\alpha_{s}^{2}\right)$ matrix element as well as with the coherent parton shower, is in good agreement with the data, although the matrix element based model is not in good agreement with other aspects of the data (see [26]). The prediction from HERWIG is slightly lower than the data, and that of ARIADNE is significantly too high. Two parton shower models without angular ordering, JETSET "incoherent" and COJETS, predict too low values of both $R$ and $R_{p}$. The closed string and independent fragmentation models are in significant disagreement with the data.

It is of interest to know whether the origin of the observed string effect is entirely at the perturbative level, or if it is also necessary to include a non-perturbative component in order to describe the data. Figure 44 again shows the measured values of $R$ and $R_{p}$ along with model predictions, in this case at both parton and hadron levels. All parton-shower models with angular ordering (i.e. all except the incoherent parton shower and COJETS) predict both $R$ and $R_{p}$ greater than unity already at the parton level, indicating a perturbative component in the effect.

The two models based on the $O\left(\alpha_{s}^{2}\right)$ matrix elements, JETSET 7.3 optimized ME with string fragmentation and independent fragmentation, predict values of $R$ and $R_{p}$ less than unity at parton level, although the string model is able to describe the data at hadron level. The decrease at parton level is understood to arise as a consequence of the minimum mass cut-off between any pair of partons, $M_{\min }$. This was set to the smallest value allowed in the program, $M_{\min }=0.1 \cdot E_{c m}=9.12 \mathrm{GeV}$.

All of the models predict that the ratios increase in the hadronization phase, although the increase is seen to be not enough for some (independent fragmentation, COJETS) and too 
charged and neutral particles combined

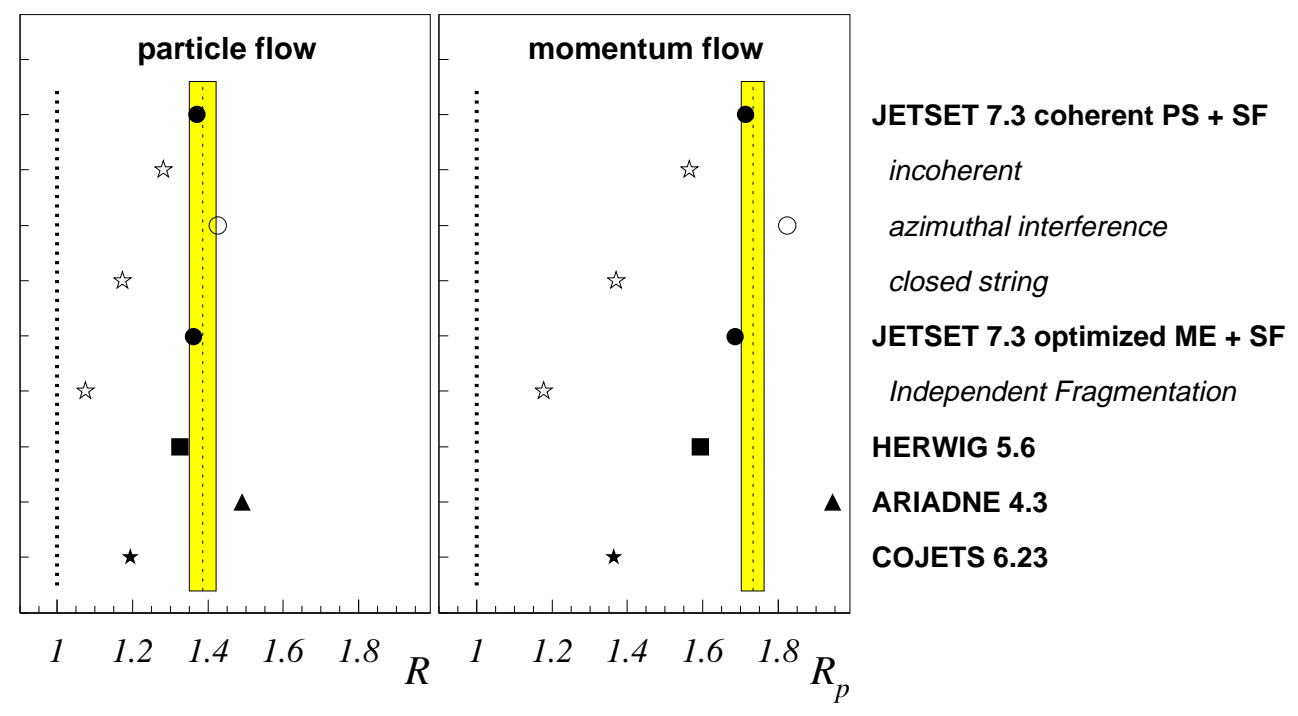

Figure 43: Measured values of $R$ and $R_{p}$ (shown as vertical bands to indicate the total error) compared to the predictions of Monte Carlo models (see text).

much for others (ARIADNE, JETSET with azimuthal interference). The high values predicted by ARIADNE are already present at the parton level. A hybrid model "HERSET" with parton shower from HERWIG and string fragmentation from JETSET, is seen to be in better agreement with the data than the HERWIG model with cluster fragmentation. From these comparisons one can see that a combination of perturbative and non-perturbative effects is necessary in order to describe the data. String fragmentation is seen to provide a highly flexible parametrization of the non-perturbative phase, successfully describing the data at hadron level for several different models at the parton level (JETSET and HERWIG parton showers, $O\left(\alpha_{s}^{2}\right)$ matrix elements).

\subsection{Charged Particle Multiplicities}

A complete understanding of the dynamics of multi-particle production in QCD is still lacking. One particularly simple observable, which contains information about the dynamics of hadron production, is the charged particle multiplicity distribution. A number of QCD models $[120,121,122]$ make predictions for the evolution of the shape and the leading moments of the multiplicity distribution as a function of the centre-of-mass energy $\sqrt{s}$.

In a first paper [79], a measurement of the inclusive charged particle multiplicity distribution observed in hadronic $\mathrm{Z}$ decays was presented. There it was shown that the multiplicity distribution for the full phase space probes the dynamics of QCD, even though it is strongly constrained through the requirement of energy-momentum and charge conservation. Later [123] the analysis was extended to study the multiplicity distribution in restricted rapidity intervals $|Y| \leq 0.5,1.0,1.5,2$ along the thrust axis, because the distribution in those limited phase space intervals is less subject to such kinematic constraints and thus can be expected to be an even more sensitive probe of the underlying dynamics of QCD. 


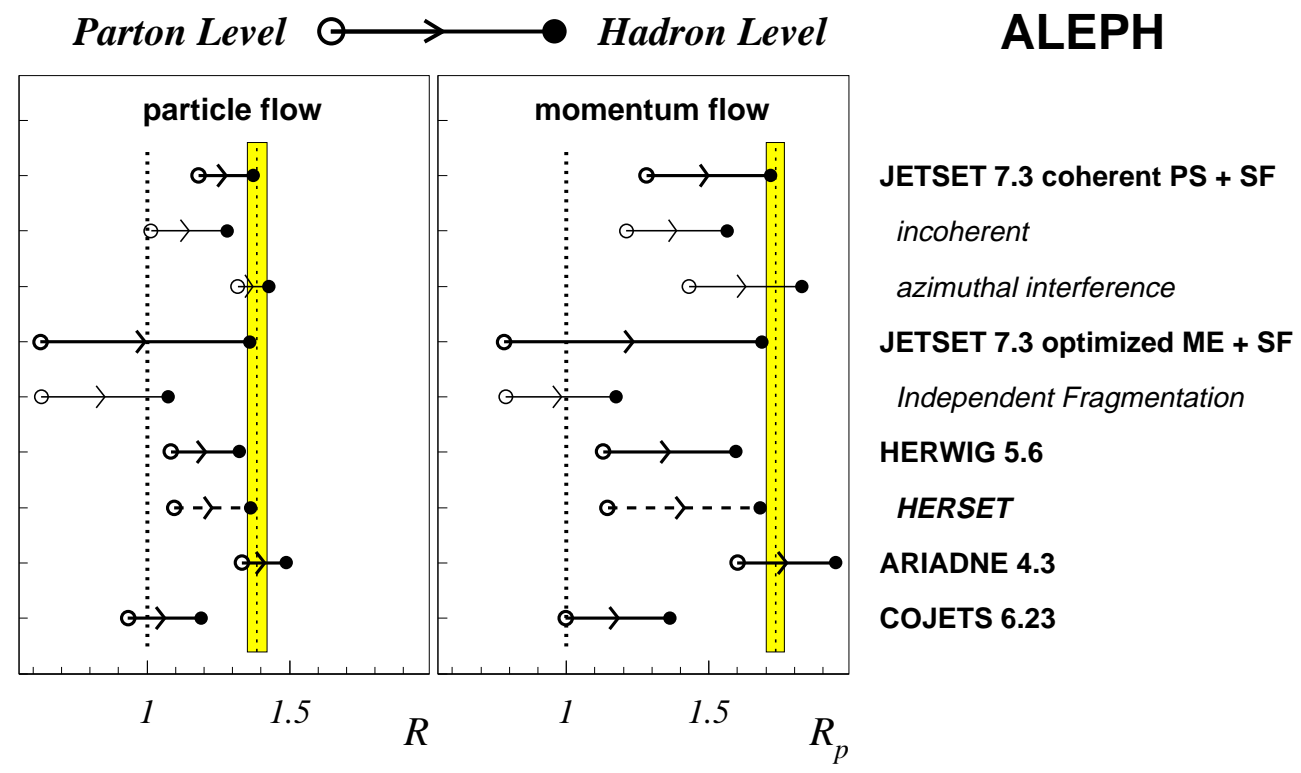

Figure 44: Measured values of $R$ and $R_{p}$ (shown as vertical bands) compared to the predictions of Monte Carlo models at parton and hadron levels (see text).

\subsubsection{Data analysis}

The following will mainly focus on the most recent paper [123], which is based on a sample of 300, 000 hadronic events at $\sqrt{s}=M_{Z}$ measured with ALEPH in 1992.

The true charged multiplicity of an event was defined as the number of charged tracks that is obtained if all particles with a mean lifetime $\tau \leq 1 \mathrm{~ns}$ decay while the others are stable. Thus, charged decay products of $\mathrm{K}_{\mathrm{S}}^{0}$ 's and strange baryons are included. Apart from decay corrections the measured charged multiplicity of an event can differ from that defined above because of acceptance losses or secondary interactions of particles with detector material. The data were corrected for the background from $e^{+} e^{-} \rightarrow \tau^{+} \tau^{-}$events, which contribute roughly $0.26 \%$ of the accepted events.

The relation between the observed multiplicity distribution $O_{i}$ in a given rapidity interval with respect to the thrust axis, and the underlying true distribution $T_{j}$ can be described by a matrix equation

$$
O_{i}=\sum_{j} G_{i j} \cdot T_{j}=\sum_{j} \widetilde{G}_{i j} \varepsilon_{j} \cdot T_{j} .
$$

The response matrix $G_{i j}$ describes distortions due to detector effects and event selection. It is defined as the probability $\varepsilon_{j}$ that an event with a true multiplicity $j$ in the rapidity interval under consideration survives the event selection cuts, times the probability $\widetilde{G}_{i j}$ to observe $i$ charged tracks instead of the true number $j$ in the same interval. The matrices $G_{i j}$ were determined from Monte Carlo simulations of a sample of $1.6 \mathrm{M}$ hadronic $\mathrm{Z}$ decays, generated with the JETSET parton shower model and fed through the full ALEPH detector simulation, reconstruction and analysis chain.

By construction, the response matrices $G_{i j}$ are independent of the relative frequencies with which events of a fixed true multiplicity $j$ are produced by the Monte Carlo generator. Therefore 
they are only weakly dependent on the actual choice of the generator. The rms spread of the measured multiplicities around the true values varies from $\approx 1.8$ to $\approx 3.7$ units when the true multiplicity goes from $n_{\text {true }}=8$ to $n_{\text {true }}=30$, almost independent of the size of the rapidity window under consideration.

Inverting Eq. (73), a model independent estimate for the true distributions $T_{j}$ was extracted from the measurements. However, trying to correct for distortions by naively inverting Eq. (73) results in instabilities due to the statistical fluctuations in the measurements (see e.g. [124]). Several ways of tackling the inverse problem Eq. (73) for finite statistics are discussed in the literature [125]. The basic idea always is to supplement the measurements by an additional constraint that stabilizes the unfolding result. In this analysis the "Method of reduced crossentropy" (MRX) [126] was used to correct the measured distribution for smearing effects. After that the "unsmeared" distribution was corrected for efficiency.

In a second analysis, parametric models were studied. Here the true distribution $T_{j}=P_{j}(\vec{\Theta})$ is given either as a function of a parameter vector $\vec{\Theta}$ or by the predictions from different Monte Carlo models. Given as input the respective true distributions $T_{j}$, the matrices $G_{i j}$ were employed as convenient means to incorporate the effect of the full detector simulation. Multiplying $T_{j}$ with the response matrix the results were compared directly with the raw measurements. In the case of parametric models the parameters were determined by a standard least squares fit.

The multiplicity distributions were corrected for the effect of initial state radiation by applying a set of bin-by-bin correction factors, determined from the JETSET model. Except for the lowest multiplicity bins this correction turned out to be entirely negligible. In the model independent analysis the corrections were applied to the unfolded distributions. For the parametric fits the effects of initial state radiation were included before folding with the response matrix.

\subsubsection{Model Independent Results}

The unfolded charged particle multiplicity distribution of hadronic Z decays is given in Table 25 . Note that as a consequence of the correction procedure, the errors are correlated. Since the selection criteria require at least five observed charged tracks per event there is no information about the probability of having a hadronic $Z$ decay with only two charged tracks. The probability for a $\mathrm{Z}$ to produce four charged tracks can still be estimated, although with large errors, because, due to smearing effects, there is still a small chance that those events pass the selection criteria.

The errors given in Tables 25 and 27 have been updated with respect to those reported in [123] in the following way. In the nominal analysis, the unfolding matrix is based on the JETSET Monte Carlo. The systematic error due to model dependence has been estimated by constructing a response matrix based on HERWIG. Since a high statistics sample of HERWIG events was not available, a complete unfolding based on this matrix was not possible. The matrix could be used in conjunction with the unfolded distribution from the nominal analysis, however, to obtain a prediction $O_{i}^{\text {HERWIG }}$ for the observed distribution according to Eq. (73). The same procedure can be done with the distribution unfolded with the JETSET-based matrix to obtain $O_{i}^{\text {JETSET }}$. (This is very similar, but not identical, to the actual observed distribution.) From each of these distributions, the mean multiplicity was determined, and the difference $\Delta n$ was taken as the systematic error in the mean due to model dependence. 
In order to propagate this uncertainty into the individual bins, the nominal unfolded distribution $P_{n}$ was convoluted with a Poisson distribution of mean $\Delta n$, to give $P_{n}^{\prime}$. The bin-by-bin difference between $P_{n}$ and $P_{n}^{\prime}$ was then taken as the systematic uncertainty due to model dependence. Although the errors obtained in this way are smaller than those reported in [123], they are still larger for certain multiplicity values, e.g. $n=4,6,8$, than what can be obtained by other measurements. The relatively large errors in this range are a consequence of the very weak assumptions made in the unfolding procedure.

Table 26 contains the results for the mean charged particle multiplicity $\langle n\rangle$, dispersion $D$, where

$$
D=\sqrt{\left\langle n^{2}\right\rangle-\langle n\rangle^{2}},
$$

and the derived quantities $\langle n\rangle / D$ and second binomial moment $R_{2}$, defined as

$$
R_{2}=\frac{\langle n(n-1)\rangle}{\langle n\rangle^{2}}=1+\frac{D^{2}}{\langle n\rangle^{2}}-\frac{1}{\langle n\rangle} .
$$

Being defined through ratios of moments, these derived quantities are infrared safe and thus can be predicted in perturbation theory. Details about the systematic uncertainties are given in $[123]$.

\begin{tabular}{|r|lll|}
\hline$n$ & \multicolumn{3}{|c|}{$P_{n}$} \\
\hline 4 & 0.0020 & \pm 0.0020 & \pm 0.0025 \\
6 & 0.0021 & \pm 0.0009 & \pm 0.0025 \\
8 & 0.0058 & \pm 0.0010 & \pm 0.0022 \\
10 & 0.0266 & \pm 0.0018 & \pm 0.0028 \\
12 & 0.0531 & \pm 0.0031 & \pm 0.0064 \\
14 & 0.079 & \pm 0.004 & \pm 0.013 \\
16 & 0.128 & \pm 0.005 & \pm 0.015 \\
18 & 0.118 & \pm 0.005 & \pm 0.018 \\
20 & 0.133 & \pm 0.005 & \pm 0.012 \\
22 & 0.122 & \pm 0.004 & \pm 0.010 \\
24 & 0.090 & \pm 0.004 & \pm 0.010 \\
26 & 0.0760 & \pm 0.0031 & \pm 0.0062 \\
28 & 0.0559 & \pm 0.0029 & \pm 0.0064 \\
30 & 0.0389 & \pm 0.0023 & \pm 0.0032 \\
32 & 0.0264 & \pm 0.0018 & \pm 0.0027 \\
34 & 0.0166 & \pm 0.0012 & \pm 0.0019 \\
36 & 0.0105 & \pm 0.0010 & \pm 0.0017 \\
38 & 0.0080 & \pm 0.0008 & \pm 0.0014 \\
40 & 0.0044 & \pm 0.0006 & \pm 0.0009 \\
42 & 0.0019 & \pm 0.0004 & \pm 0.0005 \\
44 & 0.00091 & \pm 0.00022 & \pm 0.0004 \\
46 & 0.00076 & \pm 0.00018 & \pm 0.0006 \\
48 & 0.00003 & \pm 0.00004 & \pm 0.0011 \\
50 & 0.00038 & \pm 0.00027 & \pm 0.0004 \\
52 & 0.00023 & \pm 0.00009 & \pm 0.0002 \\
54 & 0.00013 & \pm 0.00014 & \pm 0.0002 \\
\hline & & & \\
\hline
\end{tabular}

Table 25: Unfolded charged particle multiplicity distribution giving the probability $P_{n}$ to have a hadronic $\mathrm{Z}$ decay with $n$ charged particles. The first error is the statistical error, the second the systematic uncertainty. For $n=2$ no measurement was attempted. The JETSET 7.2 parton shower prediction is $P_{2}=0.00001 \pm 0.00001$. 


\begin{tabular}{|lllllll|}
$\langle n\rangle$ & $=$ & 20.91 & \pm & 0.03 & \pm & 0.22 \\
$D$ & $=$ & 6.425 & \pm & 0.031 & \pm & 0.087 \\
$\langle n\rangle / D$ & $=$ & 3.255 & \pm & 0.019 & \pm & 0.055 \\
$R_{2}$ & $=$ & 1.0466 & \pm & 0.0008 & \pm & 0.0029 \\
\hline
\end{tabular}

Table 26: Leading moments of the charged particle multiplicity distribution. The first error is the statistical error, the second the systematic uncertainty of the result.

\subsubsection{Energy Dependence of the Charged Multiplicity Distribution}

\section{KNO scaling}

Originally derived by starting from the Feynman scaling [127] behaviour for multi-particle production, the KNO [120] scaling hypothesis predicts that the shape of the multiplicity distribution plotted in the form $\langle n\rangle P_{n}$ versus $z=n /\langle n\rangle$ is independent of $\sqrt{s}$. In Fig. 45 (a) the multiplicity distribution measured in [79] and plotted in KNO form is compared to data at $\sqrt{s}=43.6 \mathrm{GeV}$ and $\sqrt{s}=29 \mathrm{GeV}$ measured by the TASSO and HRS collaborations [128, 129]. It can be seen that in the energy range $\sqrt{s}=29-91.2 \mathrm{GeV}$ the data are in remarkable agreement with the expectations from KNO scaling. Also shown is the distribution from the JETSET 7.2 parton shower model, tuned at $\sqrt{s}=91.2 \mathrm{GeV}$ [26], which provides a very good description of the data. KNO scaling further implies the ratio $\langle n\rangle / D$ to be independent of $\sqrt{s}$. Measurements of this ratio between $12 \mathrm{GeV}$ and $91.25 \mathrm{GeV}[128,130,131]$ are shown in Fig. 45 (b). The data are well described by a constant with $C_{K N O}=3.23 \pm 0.05$ with a $\chi^{2} / N D F=5.0 / 8$. The energy dependence of $\langle n\rangle / D$ is also found to be well reproduced by the JETSET model without retuning parameters, i.e. approximate KNO scaling appears to be a natural consequence of the parton shower approach to multi-particle production.

\section{The Mean Charged Particle Multiplicity}

As a consequence of the running of the strong coupling constant $\alpha_{s}$, a next-to-leading order QCD calculation [59] in the framework of the MLLA+LPHD predicts an energy dependence of the mean multiplicity of the form

$$
\left\langle n\left(E_{c m}\right)\right\rangle=K_{\mathrm{LPHD}} \cdot \alpha_{s}^{B}\left(E_{c m}\right) \cdot \exp \left(A / \sqrt{\alpha_{s}\left(E_{c m}\right)}\right)
$$

with coefficients $A=\sqrt{864 \pi} /\left(33-2 n_{f}\right) \approx 2.265$ and $B=\left(297+22 n_{f}\right) /\left(1188-72 n_{f}\right) \approx 0.4915$ for $n_{f}=5$ active quark flavours, and $\alpha_{s}\left(E_{c m}\right)$ as given in Section 1.1. The free parameters are the phenomenological normalization constant $K_{\mathrm{LPHD}}$ and the value of the strong coupling at the scale of the $\mathrm{Z}$ mass, $\alpha_{s}\left(M_{Z}\right)$. Figure 46(a) shows how the QCD prediction compares to the data, taken from a compilation of results in [132]. Taking $\alpha_{s}\left(M_{Z}\right)=0.118$ a perfect fit is obtained with $K_{\mathrm{LPHD}}=0.0822$ and $\chi^{2} / n_{\mathrm{df}}=5.2 / 20$. Also shown is the prediction from the JETSET 7.2 parton shower model with parameters fixed at $\sqrt{s}=91.2 \mathrm{GeV}$ [26], which follows the QCD curve very closely.

For the QCD prediction it has been assumed that the multiplicity of the final state is a function of only the centre-of-mass energy. This is only approximately true, because the multiplicity from primary $b$ quarks is higher than from light flavours and because the flavour composition changes between photon and $\mathrm{Z}$ mediated reactions. Assuming that the difference 

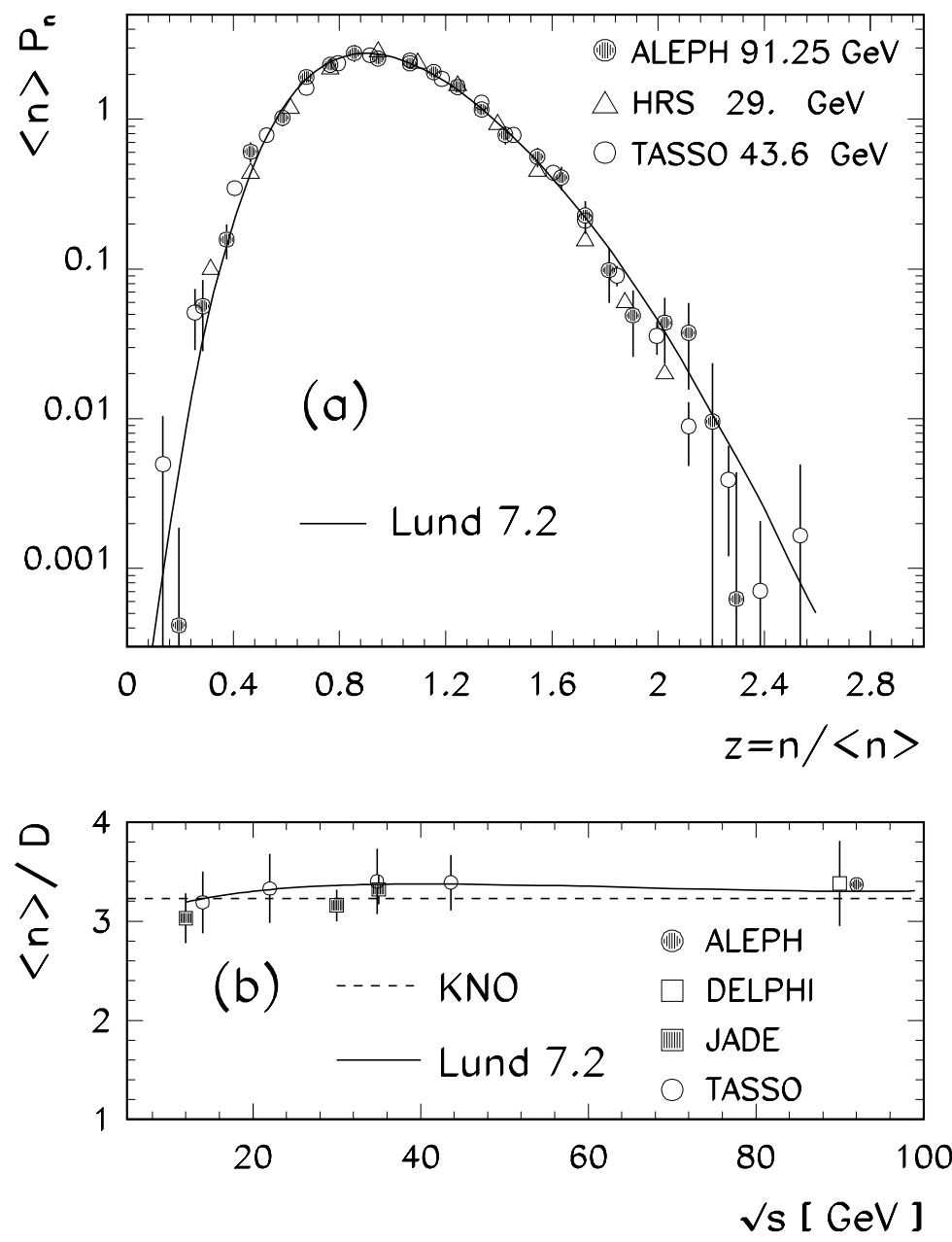

Figure 45: The unfolded charged particle multiplicity distribution [79] in KNO form (a) compared with results from the TASSO and HRS collaboration, and (b) the energy dependence of the ratio $\langle n\rangle / D$. Also shown are the predictions from the JETSET 7.2 parton shower model with parameters tuned at $\sqrt{s}=$ $91.2 \mathrm{GeV}$.

$\sqrt{ } \mathrm{s}[\mathrm{GeV}]$

in multiplicities between $b$-quark and light-quark events is energy independent as measured in [133], the resulting variation in the event multiplicity due to the change of the primary flavour composition is 0.4 tracks/event between $\sqrt{s}=12-91.2 \mathrm{GeV}$. This is smaller than the typical experimental errors and thus can be neglected.

\section{The Width of the Multiplicity Distribution}

The same QCD calculation which describes the energy evolution of the mean charged particle multiplicity also predicts the evolution of the second binomial moment $R_{2}$. One obtains in next-to-leading order

$$
R_{2}\left(E_{c m}\right)=\frac{11}{8}\left[1-C \sqrt{\alpha_{s}\left(E_{c m}\right)}\right]
$$

with

$$
C=\frac{1}{\sqrt{6 \pi}} \frac{4455-40 n_{f}}{1782} \approx 0.55 \text {. }
$$

The experimental values for $R_{2}$ are compared with the QCD prediction (MLLA+LPHD) in Fig. 46(b). For QCD the leading (11/8) and next-to-leading order predictions are plotted, with $\alpha_{s}\left(E_{c m}\right)$ calculated as above, with $\alpha_{s}\left(M_{Z}\right)=0.118$. Both curves are significantly above the data. It follows that higher than next-to-leading order QCD contributions or non-perturbative effects are needed to explain the width of the charged particle multiplicity distribution, even 


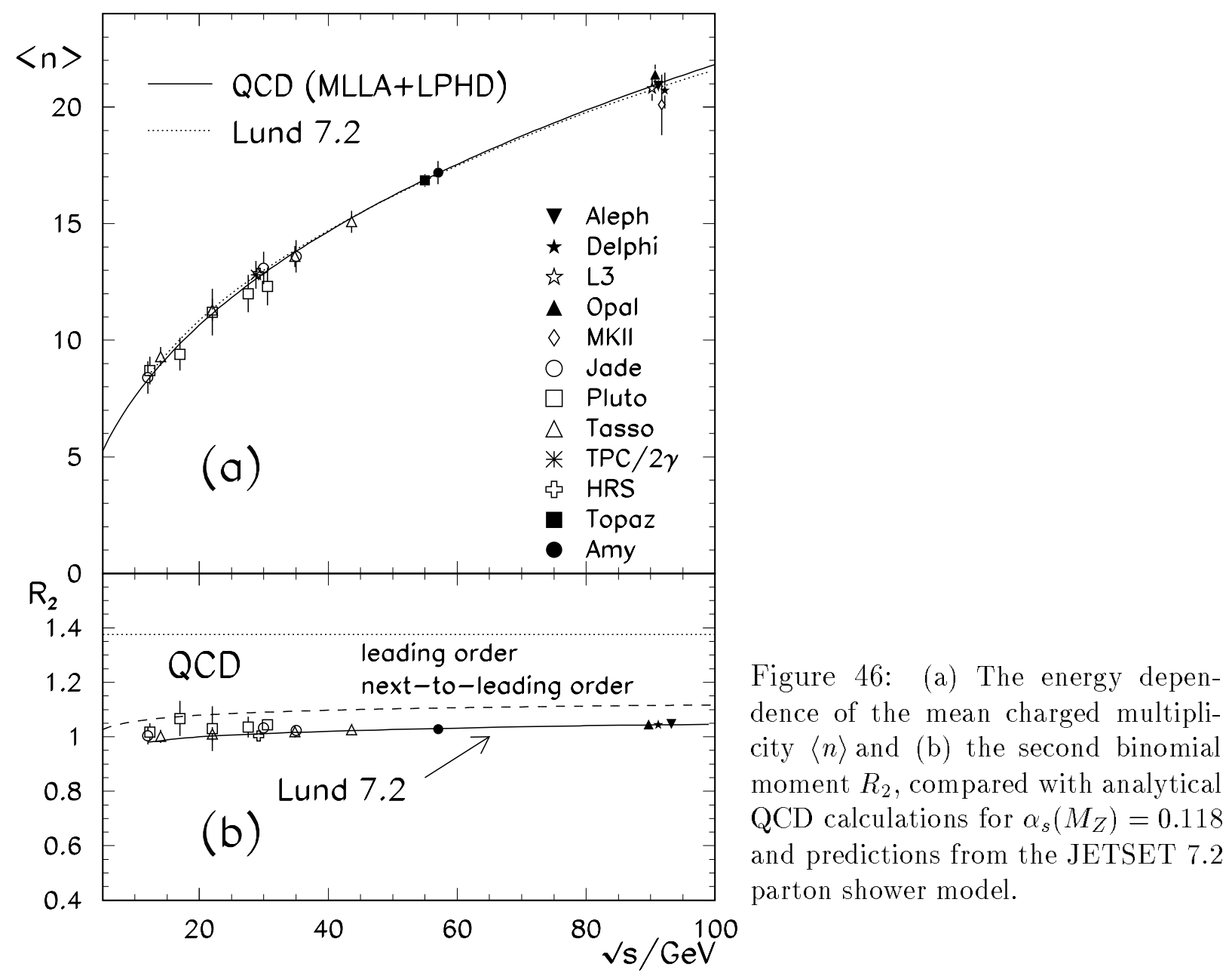

though it is remarkable the extent to which the next-to-leading order corrections do account for the bulk of the higher order effects. The JETSET 7.2 parton shower model again provides an accurate description of the measurements in the range $\sqrt{s}=12-91.2 \mathrm{GeV}$.

\subsubsection{Charged Particle Multiplicities in Rapidity Windows}

The previous discussion shows that the inclusive charged particle multiplicity distribution holds information about the dynamics of QCD. Further insight can be gained by restricting the analysis to rapidity intervals along the thrust axis, where trivial kinematical constraints like charge conservation have less impact [123]. The model independent unfolding results for the charged particle multiplicity distributions of hadronic $\mathrm{Z}$ decays in rapidity intervals $|\mathrm{Y}| \leq 0.5,1.0,1.5,2.0$ are tabulated in [123].

The unfolded distributions for the rapidity windows $|Y| \leq 0.5,2.0$ and the full window are shown in Fig. 47. For comparison, the predictions from the parton shower models JETSET 7.3, HERWIG 5.6 and the results from the parametric fits discussed below are overlayed over the experimental results. In going from small rapidity windows to the full phase space not only does the width of the multiplicity distribution grow steadily but also its shape changes significantly. It can be described by a simple curve with an always negative second derivative for very small and very large intervals. For intermediate size intervals a pronounced shoulder structure develops. This kind of structure was first observed in [134] and 


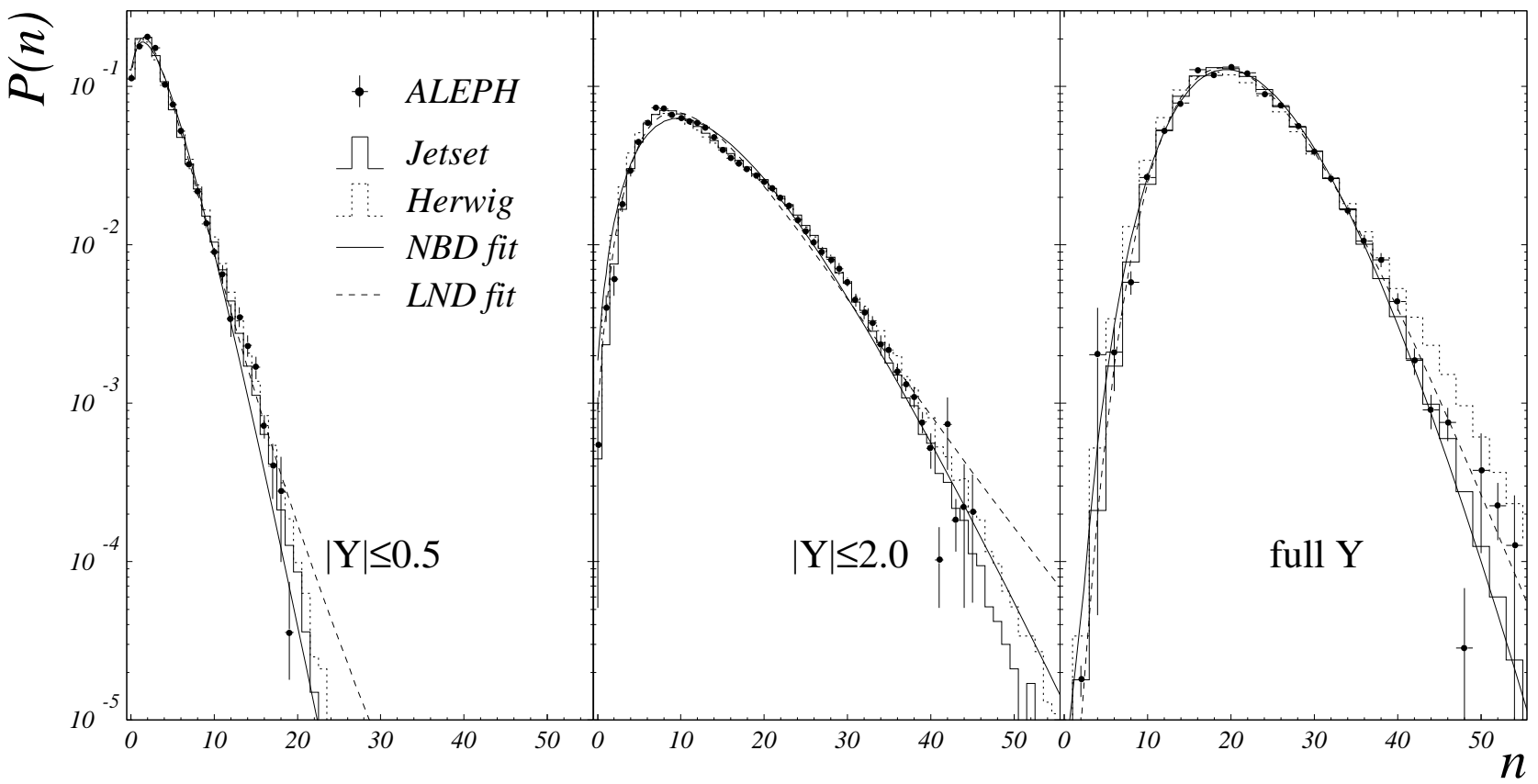

Figure 47: Unfolded charged particle multiplicity distributions for the small $(|\mathrm{Y}| \leq 0.5)$, medium $(|\mathrm{Y}| \leq 2.0)$ and full rapidity window compared to the prediction from JETSET and HERWIG and best fits to the negative-binomial and log-normal distributions. The error bars are statistical only.

points towards several independent components contributing to the charged particle multiplicity distribution, which are invisible for very small windows and average out when looking at the fully inclusive distribution covering the complete phase space. These independent components could be identified [135] with different event topologies, i.e. two-, three- and four-jet events, demonstrating that the charged particle multiplicity distribution carries information about the hard perturbative phase of multihadron production processes.

The mean charged multiplicities $\langle n\rangle$ and the dispersion $D$ from the unfolding results compared to the model predictions are summarized in Table 27. The data are found to be in reasonable agreement with the JETSET prediction, whereas the width predicted by the HERWIG model exhibits significant discrepancies.

Several parametrizations for the shape of the charged particle multiplicity distribution are discussed in the literature. Of particular interest are the negative binomial distribution (NBD) and the log-normal distribution (LND).

The NDB is defined as

$$
P_{n}(\langle n\rangle, k)=\frac{k(k+1) \ldots(k+n-1)}{n !}\left(\frac{\langle n\rangle}{\langle n\rangle+k}\right)^{n}\left(1+\frac{\langle n\rangle}{k}\right)^{-k} .
$$

Theoretically the NBD can be derived from the so-called clan model [136] for multiparticle production. Here an event consists on average of $N=k \ln (1+\langle n\rangle / k)$ clans which on average decay into $\langle n\rangle / N$ secondary particles. In the context of QCD those clans might be identified with a number of $N$ partons created in a parton showering process that hadronize into $\langle n\rangle$ final state particles. Perturbative QCD predicts, in fact, that the ratios of moments of the charged particle multiplicity distribution behave approximately like those of the NBD [121].

The LND can be derived from the general assumption that multi-particle production 


\begin{tabular}{|c|c|c|c|}
\hline & unfolded result & JETSET & HERWIG \\
\hline Y-range & \multicolumn{3}{|c|}{$\langle n\rangle$} \\
\hline $\mathrm{Y} \mid \leq 0.5$ & $3.074 \pm 0.006 \pm 0.039$ & \pm 0.003 & \pm 0.003 \\
\hline$Y \mid \leq 1.0$ & $6.436 \pm 0.009 \pm 0.081$ & $6.396 \pm 0.005$ & \pm 0.005 \\
\hline $\mathrm{Y} \mid \leq 1.5$ & $9.78 \pm 0.01 \pm 0.10$ & \pm 0.006 & \pm 0.006 \\
\hline $\mathrm{Y} \mid \leq 2.0$ & $13.01 \pm 0.01 \pm 0.12$ & $13.083 \pm 0.007$ & $12.925 \pm 0.007$ \\
\hline full Y & $20.91 \pm 0.03 \pm 0.20$ & $20.861 \pm 0.006$ & $20.695 \pm 0.007$ \\
\hline Y-range & \multicolumn{3}{|c|}{$\bar{D}$} \\
\hline $\mathrm{Y} \mid \leq 0.5$ & $2.582 \pm 0.008 \pm 0.039$ & \pm 0.003 & \pm 0.003 \\
\hline $\mathrm{Y} \mid \leq 1.0$ & $4.54 \pm 0.01 \pm 0.07$ & \pm 0.005 & \pm 0.005 \\
\hline $\mathrm{Y} \mid \leq 1.5$ & $6.082 \pm 0.013 \pm 0.053$ & \pm 0.006 & \pm 0.006 \\
\hline $\mathrm{Y} \mid \leq 2.0$ & $7.068 \pm 0.013 \pm 0.048$ & \pm 0.006 & \pm 0.006 \\
\hline full Y & $6.425 \pm 0.031 \pm 0.066$ & \pm 0.005 & \pm 0.006 \\
\hline
\end{tabular}

Table 27: Leading moments $\langle n\rangle$ and $D$ for data and MC models. Also given is the $\chi^{2} /$ bin, based only on the statistical errors.

proceeds via a scale invariant stochastic branching process [122]. Here the final state multiplicity evolves over many generations, with the multiplicity ratio between successive generations described by independent random variables $\varepsilon_{i}, n_{i+1} / n_{i}=1+\varepsilon_{i}$. In the limit of a large number of branching processes the LND follows from the central limit theorem. The discrete probability distribution $P_{n}$ for charged particle multiplicities is obtained from the continuous LND by integrating the continuous distribution over the interval $[n, n+\delta n]$,

$$
P_{n}(\mu, \sigma, c)=\int_{n}^{n+\delta n} \frac{N}{n^{\prime}+c} \exp \left(-\frac{\left[\ln \left(n^{\prime}+c\right)-\mu\right]^{2}}{2 \sigma^{2}}\right) d n^{\prime} .
$$

Here $\delta n=1$ for restricted rapidity intervals where even and odd multiplicities contribute, and $\delta n=2$ for the full phase space where charge conservation ensures that the total number of particles is always even. The LND contains three free parameters, $\mu, \sigma$ and $c$, and a normalization factor $N$. The parameters are redefined in the fitting procedure in order to reduce correlations (see $[123]$ ).

Figure 48 shows how the various estimates for the charged particle multiplicity distribution compare to the measurements in a narrow $(|\mathrm{Y}| \leq 0.5)$, a medium size $(|\mathrm{Y}| \leq 2.0)$ and the full rapidity window. In all cases the estimates for the true distribution were folded with the response matrix and then compared directly with the uncorrected data. The differences are shown in Fig. 48. For the unfolded data this constitutes a cross check of the procedure. For the four models the quality of the description of the data varies from being indistinguishable from the unfolded data to having a significant disagreement. The error bars reflect the statistical and systematic errors combined in quadrature; systematic uncertainties dominate.

The results clearly show that the NBD does not describe the data, either in restricted rapidity intervals or for the full phase space. The LND does fit the data for very small rapidity intervals, $|\mathrm{Y}| \leq 0.5$, and the full window, but fails to do so for intermediate size intervals. Intuitively this can be understood from the fact that multi-jet effects mostly affect those medium size intervals, and it is not surprising that simple parametrizations like the LND or NBD fail when several components like two-, three- or multi-jet events contribute. In contrast to the simple parametric models, both the JETSET and the HERWIG parton shower models 


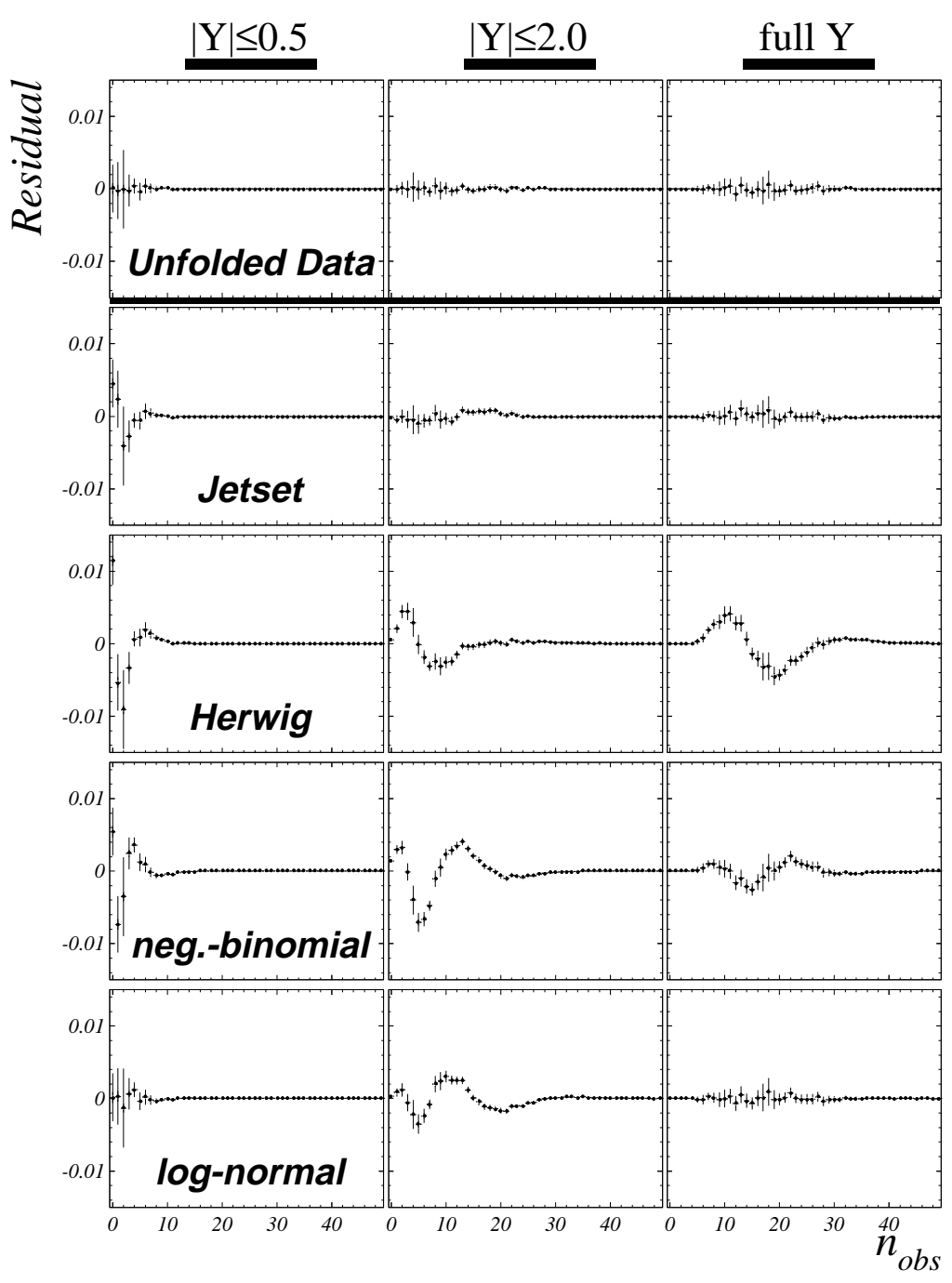

Figure 48: Differences between data and various models for small $(|Y| \leq 0.5$, left $)$, medium $(|Y| \leq$ 2.0, middle) and the full rapidity window (right). The error bars reflect the statistical and systematic errors combined in quadrature; systematic uncertainties dominate.

reproduce the shoulder structure in the multiplicity distributions for intermediate size rapidity intervals. It is, however, interesting to note that of the two parton-shower models studied here only the JETSET model gives a good quantitative description of the charged particle multiplicity distribution in all rapidity intervals.

\subsection{Intermittency}

Intermittency is a term derived from turbulence theory [137] and introduced to particle physics to describe non-poissonian fluctuations observed in some distributions [138]. Such non-poissonian fluctuations are observed in different experiments (deep inelastic scattering, hadron collisions, heavy-ion collisions, $e^{+} e^{-}$) and have different origins. In $e^{+} e^{-}$annihilations the origin is well understood as a result of studies as described below. In this case gluon activity was shown $[139,140]$ to be the main origin for the behaviour of intermittency moments, and the effects were successfully described analytically within the DLA and MLLA frameworks [141].

For the studies in ALEPH [139, 140], the charged particles were analysed using the data collected in 1989-1990. To measure fluctuations of the number of particles $n_{m}$ per bin in a region of phase space divided equally into $M$ bins, the following quantities were found for each bin $m:\left(S_{i}\right)_{m}=n_{m}\left(n_{m}-1\right) \ldots\left(n_{m}-i+1\right)$. The variable studied was rapidity $y$ using first the 
differential moments [139], defined by

$$
\left(f_{i}\right)_{m}=\frac{\left\langle\left(S_{i}\right)_{m}\right\rangle}{\langle N\rangle^{i}}
$$

where the average is taken over all events. This quantity is sensitive to the fluctuations in the $m$ th bin from event to event. The intermittent behaviour was further examined [140] using the standard factorial moments $F$ of order $i$,

$$
F_{i}(M)=\frac{\left\langle\frac{1}{M} \sum_{m=1}^{M}\left(S_{i}\right)_{m}\right\rangle M^{i}}{\langle N\rangle^{i}} .
$$

The moments in Eqs. (76) and (77) average to unity if the fluctuations of particles in the bins are Poissonian. Higher moments are sensitive to larger clusters of particles within the bins and include information contained in the lower moments. A power-law dependence of $F_{i}(M)$ with respect to $M$ hints at self-similar processes in the generation of particles. The data are shown in Fig. 49 for one-dimensional factorial moments $F_{2}(M)$ in $y$.

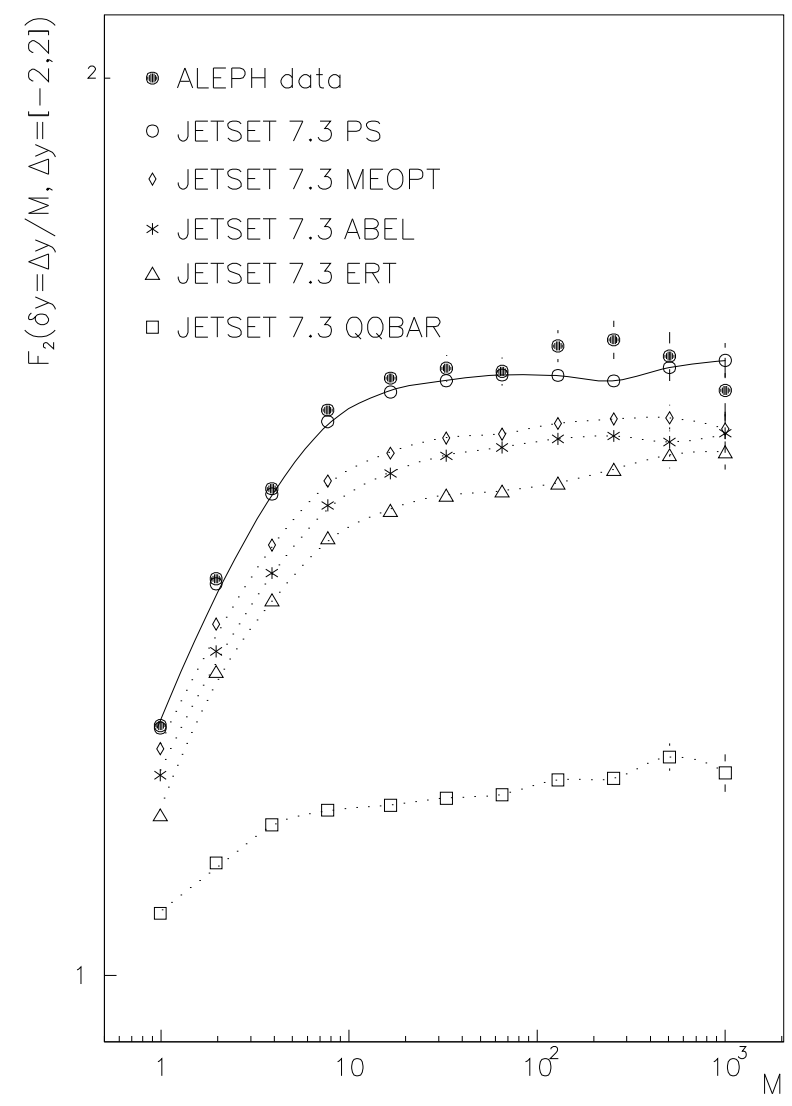

Figure 49: The second factorial moment $F_{2}(M)$ in rapidity space. Comparison of different Monte Carlo models with varying amounts of parton cascading. The solid line is to guide the eye for the default JETSET Monte Carlo prediction and the dotted lines for other versions of the model.

Using the differential moments of Eq.(76), it could be clearly demonstrated that the behaviour of the intermittency moments is mainly due to the emission of hard gluons [139]. For example, the third differential moment rises to about eight at $|y| \simeq 1$, which contains, in addition to particles from two-jet events, an enhanced activity from three-jet events. That is, the multijet structure of $e^{+} e^{-} \rightarrow$ hadrons causes the rapidity distribution of the charged particles to vary with the event topology and, for example, the moments to $F_{2}(M)$ to increase with decreasing bin size as seen in Fig. 49. If events of a given topology are selected (e.g., with a cut in thrust), the differential and factorial moments in one dimension remain close to unity when decreasing the bin size. 
The comparison [140] is shown in Fig. 49 of ALEPH data with five models available within the Lund parton shower Monte Carlo, JETSET 7.3 [25]:

PS: The full (default) parton shower model. This gives an average of 7.8 partons before the onset of string fragmentation.

ERT: The model with second order matrix elements according to Ellis, Ross, and Terrano [16]. This allows up to four partons before string fragmentation.

MEOPT: The second order matrix elements model with optimized renormalization scale. The latter improves the agreement of ERT with the experimental four-jet rate.

ABEL: Abelian parton shower model. The triple gluon vertex has been switched off in the otherwise full shower development.

QQBAR: A model with no gluons. The primary quark-antiquark-pair are hooked together with a straight string which then fragments according to JETSET 7.3. Only two partons are allowed.

All models except QQBAR and ABEL were fit to a set of ALEPH inclusive and exclusive distributions [26], which do not include the factorial moments. In the cases of QQBAR and ABEL the fragmentation parameters were adjusted to reproduce the measured average thrust and multiplicity.

It is seen in Fig. 49 that the moments with only two quarks indicate nearly Poissonian behavior (QQBAR). The main mechanism driving the moments upward is the emission of one or two hard gluons (ERT, MEOPT). The full shower cascade (PS) is necessary to fully describe the data. The cascading is insufficient without the triple gluon vertex (ABEL).

\subsection{Subjet Structure of Hadronic Events}

Analyses using subjets have proven to be useful in order to investigate the internal structure of quark and gluon jets. The general procedure followed in this section was first introduced in Ref. [142]. Subjets in two- and three-jet events are defined in the following way. First, jets are defined using an iterative clustering procedure (the Durham algorithm [28]), as described in Section 2.1.

Two- and/or three-jet events are selected using an initial value of the resolution parameter, $y_{c u t}=y_{1}$. In order to investigate the jets' internal structure, the particles of the individual jets are clustered using a smaller value of the resolution parameter $y_{0}\left(<y_{1}\right)$ so that subjets are resolved. QCD predicts differences in the subjet structure of quark and gluon jets. In the parton shower picture, these differences are understood to arise mainly from the different colour factors which enter into the probabilities for a gluon to emit another gluon, proportional to $C_{A}=3$, and the probability for a quark to emit a gluon, proportional to $C_{F}=4 / 3$. As long as the subjet resolution parameter $y_{1}$ is sufficiently large, one expects a direct correspondence between the parton level predictions of QCD and hadron level measurements.

In a first analysis, the subjet multiplicity of two- and three-jet events is measured without determining whether the jet originated from a quark or from a gluon. Similar studies have been reported in $[143,144]$. In a second analysis, gluon jets are identified by requiring evidence of long-lived hadrons containing $b$ or $c$ quarks in two jets out of a three-jet event, and the remaining jet is then taken as a gluon jet candidate. By combining the results from this jet sample with those where all jets are used, the individual subjet structure of quark and gluon jets can be inferred. 


\subsubsection{Subjet Structure of Two- and Three-Jet Events}

Although QCD makes specific predictions for identified quark and gluon jets, it is difficult experimentally to identify jets as such. An analysis was therefore carried out to measure the subjet multiplicity in two- and three-jet events, $M_{2}$ and $M_{3}$, without quark or gluon jet identification. The two-jet sample consists predominantly of an initial $q \bar{q}$ system followed by soft gluon radiation, whereas the three-jet sample is enriched by events with a single hard (high $k_{\perp}$ ) gluon. The initial $q \bar{q}$ or $q \bar{q} g$ system continues to radiate gluons, whereby the quarkgluon coupling is proportional to the QCD colour factor $C_{F}=4 / 3$, whereas the gluon-gluon coupling is proportional to $C_{A}=3$. Thus one expects the three jet sample to have a higher subjet multiplicity than the two-jet sample. For asymptotically high energies and small $y_{0}$ one expects $[142]$

$$
R=\frac{M_{3}-3}{M_{2}-2} \rightarrow \frac{2 C_{F}+C_{A}}{2 C_{F}}=\frac{17}{8}
$$

The ratio as defined here is equivalent to $M_{3} / M_{2}$ in the limit that the multiplicities are large. Subtracting the initial number of jets leads to a faster expected convergence to the asymptotic prediction of (78), and simplifies the interpretation of the results in the region where $y_{0}$ is close to $y_{1}$ (i.e. $M_{2}$ only slightly larger than two, $M_{3}$ only slightly larger than three).

The measurements of $M_{2}$ and $M_{3}$ presented here are based on charged particles only. The track and event selection criteria are described in Section 1.3.1. Using data collected in 1992 yielded a sample of approximately 300,000 hadronic events at an energy of $E_{c m}=91.2 \mathrm{GeV}$.

The measurements have been corrected for detector related effects using multiplicative correction factors $C$ as described in Section 1.3.2. These factors are computed as a function of the subjet resolution parameter $y_{0}$, and are applied to the subjet multiplicity minus the number of jets selected with $y_{c u t}=y_{1}$, i.e.

$$
\left(M_{n}\left(y_{0}\right)-n\right)_{\text {corrected }}=\left(M_{n}\left(y_{0}\right)-n\right)_{\text {measured }} \cdot C\left(y_{0}\right)
$$

with $n=2,3$. In order to minimize systematic errors, the correction factors have been computed so as to take into account charged particles only. Comparisons with Monte Carlo models are then also made using only charged particles. The correction factors do not depend strongly on $y_{0}$ and are typically in the range $1.00<C<1.12$.

In order to estimate the systematic uncertainty from the generator dependence of the correction factors, approximate factors were derived from several Monte Carlo models, as described in Section 1.3.2. The approximate correction factors reproduced the overall form of the factors based on the full detector simulation, and they differed among each other by typically less than $1 \%$ to $2 \%$. It was checked that the corrected jet multiplicities are not sensitive to the event and track selection criteria by varying all of the cuts. No evidence for a systematic dependence was found beyond the one percent level.

Based on the studies of the generator dependence of the correction factors and variation of the cuts, an overall systematic error of $2 \%$ is assigned to the mean subjet multiplicities $M_{2}-2$ and $M_{3}-3$. This is conservative for small $y_{0}$, where the subjet multiplicity tends toward the charged particle multiplicity for the event sample in question. (In Section 4.2 it is shown that the mean charged multiplicity is measured in ALEPH with a systematic error of about 1.2\%.) For the ratio $R=\left(M_{3}-3\right) /\left(M_{2}-2\right)$, the systematic errors largely cancel. The remaining 
systematic error is conservatively estimated to be around $1 \%$. On all plots the quadratic sum of statistical and systematic uncertainties is shown.

Figure 50 shows the quantities $M_{2}-2$ and $M_{3}-3$ compared to the predictions of the Monte Carlo models JETSET, HERWIG and ARIADNE. In addition, a toy model based on JETSET is shown in which the effective colour charge for the parton splitting $g \rightarrow g g$ has been reduced from the standard value predicted by $\mathrm{QCD}$ of $C_{A}=3$ to the value $C_{F}=4 / 3$, i.e. the same as for the branching $q \rightarrow q g$.


Figure 50: (a) The mean subjet multiplicities in a two-jet sample minus two as a function of the subjet resolution parameter $y_{0}$. (b) The ratio model over data. (c) Subjet multiplicities minus three and $(d)$ ratio of model over data for the three-jet sample.

As can be seen from Fig. 50 (a) and (b), all of the models, including the toy model, are in good agreement with the data for the subjet multiplicity in two-jet events $\left(M_{2}-2\right)$ over essentially the entire range of the subjet resolution parameter $y_{0}$. For the three-jet sample, however, (Fig. 50 (c) and (d)) significant discrepancies between models and data are seen for values of $y_{0}$ in the range $10^{-2}-10^{-3}$, especially for the toy model.

The agreement for $M_{2}-2$ is to be expected, since to leading order subjet production in the two-jet sample results from gluon radiation from a high energy quark, and hence is primarily sensitive to the colour factor $C_{F}=4 / 3$, related to the branching $q \rightarrow q g$. In the three-jet sample, however, subjets can also result from the splitting $g \rightarrow g g$, for which the colour charge $C_{A}$ is set to a lower value in the toy model. The default JETSET model also shows significant discrepancies for $M_{3}-3$, while HERWIG and ARIADNE provide a much better description of the data. Since these models are all essentially equivalent up to leading order at the parton level, one sees that the quantity $M_{3}-3$ is a sensitive probe of higher order effects, including in particular the effect of soft gluon radiation from a hard gluon.

If the logarithm of $1 / y_{1}$ can be considered to be large compared to unity, an analytical formula based on the resummation of next-to-leading logarithms of $1 / y_{1}$ (NLLA) [142] can be 
used to predict the subjet multiplicities. Figure 51 shows the ratio $\left(M_{3}-3\right) /\left(M_{2}-2\right)$ for twoand three- jet samples selected with $-\ln y_{1}=5\left(y_{1}=0.00674\right)$ along with the prediction of the NLLA formula, for which the effective QCD scale parameter $\Lambda$ (which cannot be directly identified with $\Lambda_{\overline{M S}}$ ) was taken to be $0.5 \mathrm{GeV}$. For purposes of comparison the formula given in [142] can be evaluated with the colour factor $C_{A}$ set to $4 / 3$. As expected, this leads to a less rapid rise in the ratio as $y_{0}$ decreases.

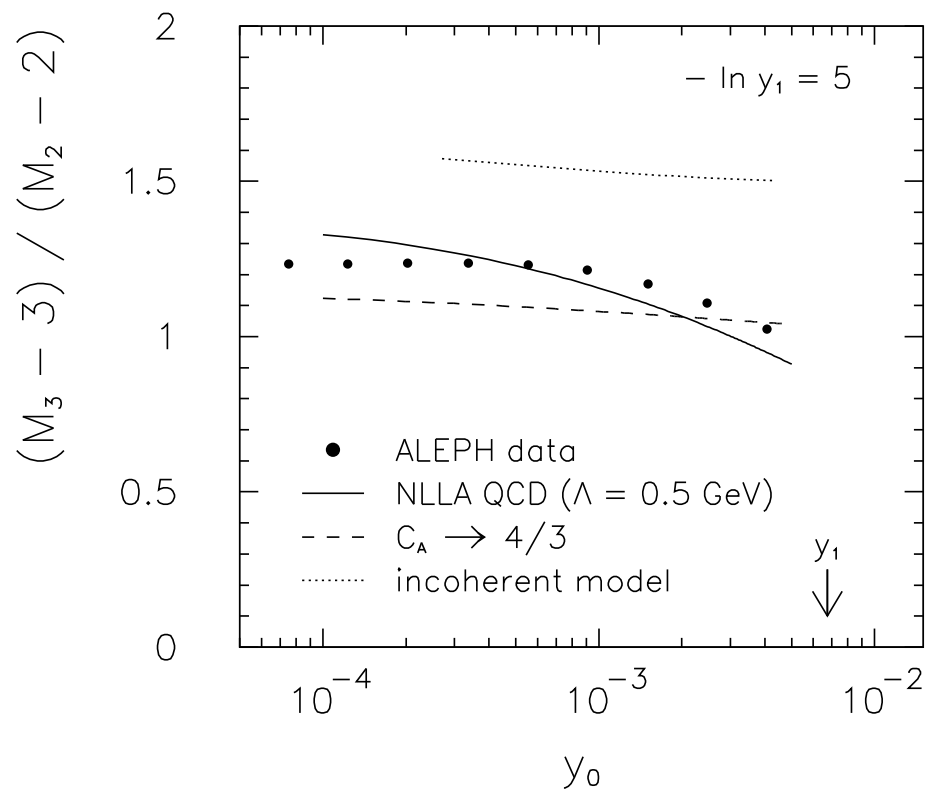

Figure 51: The ratio $\left(M_{3}-3\right) /\left(M_{2}-2\right)$ for samples of two- and three-jet events selected with $-\ln y_{1}=5\left(y_{1}=0.00674\right)$. Also shown are the predictions of NLLA QCD with the usual colour factors $C_{A}=$ 3 and for comparison with the $C_{A}$ set equal to $4 / 3$. In addition an incoherent model is shown (see text).

To further investigate the role of coherence in $\left(M_{3}-3\right) /\left(M_{2}-2\right)$, the following incoherent model has been proposed [145]. For $M_{3}$, a three-parton system is generated according to the first order matrix element, giving values of $x_{q}, x_{\bar{q}}$ and $x_{g}$, where $x_{i}=2 E_{i} / E_{c m}$. Each parton is assumed to radiate according to its energy, without interference:

$$
\begin{aligned}
& M_{2}=2 N_{q}\left(E_{c m}\right), \\
& M_{3}=N_{q}\left(x_{q} E_{c m}\right)+N_{q}\left(x_{\bar{q}} E_{c m}\right)+N_{g}\left(x_{g} E_{c m}\right),
\end{aligned}
$$

where the parton multiplicities from quark and gluon jets, $N_{q}$ and $N_{g}$, are computed in reference [142]. As can be seen from Fig. 51, the incoherent model gives a significantly higher value of $\left(M_{3}-3\right) /\left(M_{2}-2\right)$. This indicates that the subjet multiplicity of a jet is not simply given by the jet's energy, but that it also is influenced by the other colour charges in the system.

\subsubsection{Subjet Structure of Identified Quark and Gluon Jets}

In a second analysis using subjets, the properties of identified quark and gluon jets in three-jet events were investigated [146]. Here, information on charged and neutral particles was used by means of the energy flow algorithm described in Section 1.3. Three-jet events were selected using an initial value of the resolution parameter $y_{1}=0.1$. This leads to three well separated jets of approximately equal energy. Out of approximately one million hadronic events in the 1992-93 data sample, 28350 three-jet events were selected. The set of all jets in this sample is assumed to be composed of $2 / 3$ quark jets and $1 / 3$ gluon jets, which comprise the so-called mixed jet sample. 
A second set of jets is obtained by requiring evidence of long-lived hadrons in two of the three jets (indicating $b$ or $c$-quark jets) based on precision tracking information from the silicon vertex detector. These two jets are rejected and the third is taken as a candidate gluon jet (the so-called tagged sample). The gluon jet purity of the tagged sample was estimated to be $94.6 \%$.

Before determining the subjet multiplicities (minus one) $\left\langle N_{q(g)}-1\right\rangle$ and $n$-subjet rates $R_{n}$ for pure samples of quark and gluon jets, the measurements for the tagged and mixed jet samples were corrected for detector effects according to the procedure described in Section 1.3. Corrected observables for the tagged and mixed jet samples, $X_{t a g}$ and $X_{m i x}$ are related to the corresponding quantities for pure quark and gluon jets $X_{q}$ and $X_{g}$ by the following equations:

$$
\begin{aligned}
X_{\text {tag }} & =p_{\text {tag }} X_{g}+\left(1-p_{\text {tag }}\right) X_{q} \\
X_{\text {mix }} & =p_{\text {mix }} X_{g}+\left(1-p_{\text {mix }}\right) X_{q}
\end{aligned}
$$

where $p_{t a g}=0.946$ and $p_{m i x}=1 / 3$ are the gluon-jet purities in the tagged and mixed samples and $X$ represents either the mean subjet multiplicity minus one or the $n$-subjet rates for $n=2,3,4,5$. By solving these equations the desired quantities for pure quark and gluon jets can be extracted.

Figure 52 shows the ratio $r=\left\langle N_{g}-1\right\rangle /\left\langle N_{q}-1\right\rangle$ as a function of the subjet resolution parameter $y_{0}$, along with the predictions of several Monte Carlo models: JETSET, HERWIG, ARIADNE, NLLjet, and the toy model based on JETSET in which the colour factor $C_{A}$ was reduced from 3 to $4 / 3$. The ratio $r$ is measured to be $1.96 \pm 0.13 \pm 0.07$ at $y_{0}=2 \cdot 10^{-3}$, but falls to $1.29 \pm 0.02 \pm 0.01$ for $y_{0}=1.6 \cdot 10^{-5}$.

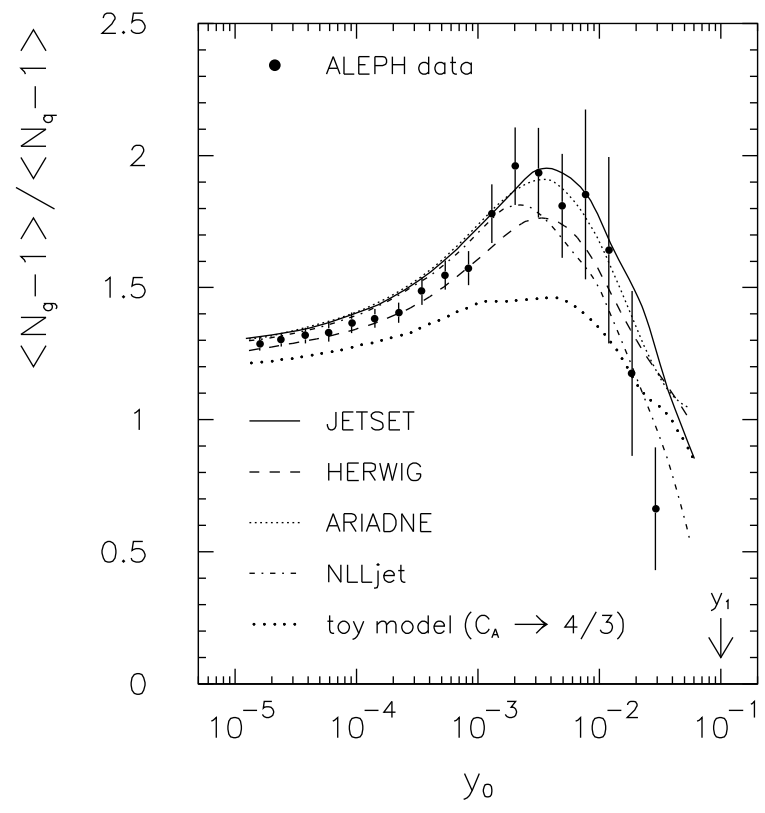

Figure 52: Measured ratios of subjet multiplicities minus one for gluon and quark jets (points) with the predictions of various Monte Carlo models (curves) as a function of the subjet resolution parameter $y_{0}$.

At intermediate values of $y_{0}$ around $10^{-3}$, one sees a large dependence of $r$ on the colour charge $C_{A}$. This corresponds to the subjets being separated by a relative transverse momentum $k_{\perp}$ of approximately $\sqrt{y_{0}} \cdot E_{c m} \approx 3 \mathrm{GeV}$. In this region, the data are in good agreement with the QCD based models, and in significant disagreement with the toy model. For lower $y_{0}$, the relative $k_{\perp}$ between subjet pairs decreases, corresponding to an increase in the effective strong coupling $\alpha_{s}\left(k_{\perp}\right)$ and a breakdown of the perturbative description. Non-perturbative effects then become important, and the sensitivity of the ratio on the colour factor $C_{A}$ is reduced (see 
[146]). At values of $y_{0} \approx 10^{-5}$, all of the models, including the toy model, predict values for $r$ in the range $1.2-1.3$.

Similar information can be obtained from the $n$-subjet rates $R_{n}$ shown in Fig. 53 along with the predictions of JETSET, HERWIG and the toy model. For quark jets, the toy model differs little from the QCD based predictions, which is to be expected since the branching $q \rightarrow q g$ depends on the colour factor $C_{F}=4 / 3$. For gluon jets, however, the rates predicted by the toy model are shifted to lower values of $y_{0}$, in significant disagreement with the data. Figures 53(a) and 53(b) show significant differences between quark and gluon jets, with e.g. gluon and quark two-subjet rates measured to be $R_{2}^{g}=0.496 \pm 0.017 \pm 0.016$ and $R_{2}^{q}=0.270 \pm 0.009 \pm 0.008$ at $y_{0}=2 \cdot 10^{-3}$, giving a ratio $R_{2}^{g} / R_{2}^{q}=1.83 \pm 0.12 \pm 0.11$.


Figure 53: Measured $n$-subjet rates for (a) quark jets and (b) gluon jets (points) as a function of the subjet resolution parameter $y_{0}$. Also shown are the predictions of various Monte Carlo models.

\subsection{Properties of Tagged Jets in Symmetric Three-Jet Events}

According to QCD, because of their larger colour charge, gluon jets are expected to have softer particle energy spectra and to be wider than quark jets of the same energy. At leading order and asymptotic energies one expects the multiplicity ratio between pairs of back-to-back gluon and quark jets to be equal to the ratio of the Casimir factors $C_{A} / C_{F}=9 / 4$. At present energies this simple prediction is significantly altered by QCD coherence effects, which strongly suppress the fragmentation of the gluon jet in the three-jet topology [142]. These predictions refer to the parton jets. Extrapolation to the final state hadrons relies on the Local Parton Hadron Duality assumption that the multiplicity of hadrons is proportional to that of the partons.

In this analysis the properties of $24 \mathrm{GeV}$ gluon and quark jets from one-fold symmetric threejet events were studied, allowing a comparison of quark and gluon jets in otherwise identical 
environments in a model-independent way. Two sets of comparisons were performed. The first one involved gluon tagged jets and quark jets whose flavour composition was determined by the electroweak couplings of the Z. The second comparison involved gluon jets and $b, c$ and $u d s$ jets separately, in an attempt to examine the effects of the different quark flavour. Gluon jet identification is achieved through identifying quark jets by means of $b$ tagging. Jets originating from $b$ quarks were identified using an impact parameter lifetime tag or a high transverse momentum lepton tag. Jets originating from $c$ quarks were identified by the presence of a fast $D^{*}$.

The properties studied are mean charged particle multiplicity, fragmentation function, rapidity distribution, and multiplicity and energy fraction within a given jet cone. The results are compared with JETSET 7.3 and HERWIG 5.5 model predictions.

\subsubsection{Data Analysis}

The standard ALEPH hadronic event selection was applied to the 1992, 1993, and 1994 data $\left(\approx 3\right.$ million events). The $k_{\perp}$ (Durham) clustering algorithm, with the E recombination scheme and a jet resolution parameter of $y_{c u t}=0.01$ was applied to all energy flow objects to select three-jet events. Jets were required to have a polar angle greater than $40^{\circ}$ with respect to the beam axis.

The jets were projected on to the event plane which was defined according to the quadratic momentum tensor. One-fold symmetric configurations were selected by requiring that the angles in the event plane between the highest energy jet and each of the two lower energy jets were in the range $150^{\circ} \pm 7.5^{\circ}$. This kinematic configuration implied that the mean energy of each of the two lower energy jets was $24.7 \mathrm{GeV}$ for quark jets and $24.0 \mathrm{GeV}$ for gluon jets. These criteria were satisfied by 22640 events.

Symmetric event configurations have been previously used in various analyses of quark and gluon jets $[147,148]$. The one-fold symmetric configuration employed here guarantees a large energy difference between the most energetic jet (J1) and the two other (J2, J3). Hence J1 has a high probability of originating from a quark or anti-quark. The Monte Carlo estimate is that in only $3 \%$ of the events is $\mathrm{J} 1$ a gluon jet.

The mixture of J2 and J3 jets from all events constituted the mixed sample, M, containing almost half quark and half gluon jets of equal energies. The quarks are a mixture of flavours determined by the electro-weak couplings of the Z referred to as "natural flavour mix", NFM. If for a given event one of the two lower energy jets has a high probability to be a $b$ jet, the remaining jet is identified as a gluon jet and enters into the gluon tagged sample, T. Tagging only the highest energy jet as a $b(c)$ jet, the two lower energy jets are equally likely to be the other $b(c)$ jet or the gluon jet. The corresponding sets of events are referred to as $\mathrm{B}$ sample and $\mathrm{C}$ sample respectively, which were equivalent to the $\mathrm{M}$ sample except for the flavour composition of the quark jets. Figure 54 shows the four sample types used.

Jets originating from a $b$ quark can often be identified via the characteristics of the decay of a $B$ hadron: the presence of a secondary vertex or the presence of a high transverse momentum lepton. The lifetime tag is described in Section 1.3. A jet (J3) was tagged as a gluon jet and included in the $\mathrm{T}$ sample if the other jet (J2) had a high probability of being a $b$ jet. The cut chosen here resulted in a T sample of 2071 jets with an estimated gluon purity $P_{g}^{T}=0.90$. The $\mathrm{B}$ sample was selected using a lepton tag based on the standard ALEPH lepton selection [149, 36]. Jets were tagged as $b$ jets if they contained a lepton with momentum greater than $3 \mathrm{GeV} / c$ and a transverse momentum with respect to the jet greater than $1.25 \mathrm{GeV} / c$, where the jet 


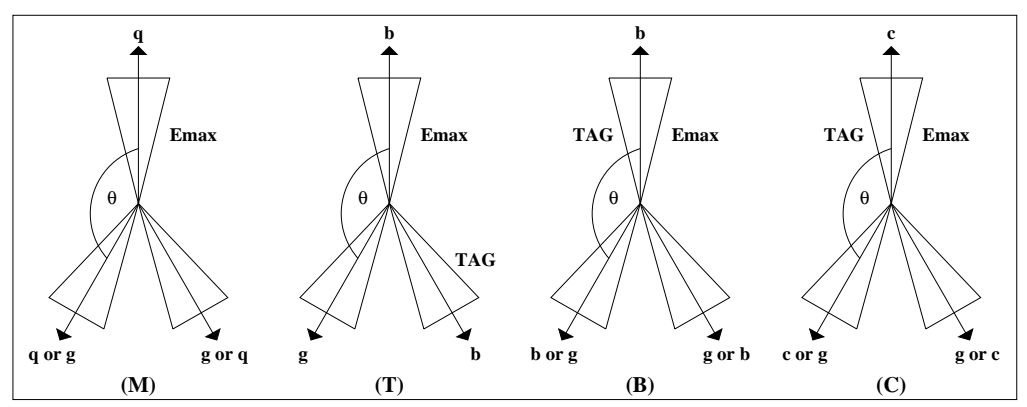

Figure 54: The four tagging configurations: the $50 \%$ quark-gluon (M)ixed sample, the gluon (T)agged sample, and the (B)-quark and (C)quark enriched mixed samples.

direction was determined without the lepton. A lepton in the highest energy jet defines the B sample, which contains 436 events and the flavour composition is: $88 \% b, 6.1 \% \mathrm{c}$ and $5 \% \mathrm{uds}$. The final event sample, $\mathrm{C}$, was selected by requiring the presence of a high momentum $D^{*}$ in the highest energy jet. The $D^{*}$ were reconstructed via their $D^{*} \rightarrow D^{0} \pi$ decay with subsequent $D^{0} \rightarrow K \pi$ decays, as described in [150]. Their energy was required to be more than $20 \mathrm{GeV}$. The selected sample consists of $70 \pm 3 \% c$ events and no light quark events [151]. From the 22640 symmetric events, only 20 contained such a fast $D^{*}$.

\subsubsection{Unfolding of the Jet Properties}

The analysis is based on the comparison of jets which have had no tagging criteria applied directly to them. Hence the bias introduced by the tagging method is kept to a minimum. Pure quark and gluon jet properties can be extracted from the four samples via a simple unfolding procedure if the quark flavour composition and the gluon purity of the samples are known. These parameters were estimated from JETSET Monte Carlo events.

The gluon purity, defined as the ratio of the number of correctly tagged gluon jets over the number of jets tagged as gluon jets, was estimated from Monte Carlo events using the procedure described in [152] to relate each reconstructed jet to its parent parton. For the chosen topology a unique assignment was found in $99 \%$ of all cases.

For a jet sample whose gluon purity and fractions of the different quark flavours have been estimated to be $P_{g}$ and $P_{u d s}, P_{b}, P_{c}$, respectively, the measured value of an observable $A$ may be expressed as

$$
A=P_{g} A_{g}+P_{u d s} A_{u d s}+P_{c} A_{c}+P_{b} A_{b},
$$

where $A_{g(q)}$ is the corresponding value for pure gluon (quark) jets. The four different samples M, T, B and C yield four such equations which can be solved simultaneously allowing a direct comparison of the properties of the gluon and the different flavour quark jets. The high statistics samples, $\mathrm{M}$ and $\mathrm{T}$, can also be used together as the quark flavour composition of the $\mathrm{T}$ sample was tuned to be similar to the M composition, to relate gluon jet and NFM quark jet properties.

Ideally, the samples should consist of events where the jets are produced in the same kinematical configurations and the tagging procedure should not introduce a bias. While (almost) identical kinematics are ensured by using symmetric events, the existence of a tagging bias cannot be excluded. Using JETSET Monte Carlo events, bias corrections factors were determined by comparing the value of the observable $A_{g(q)}$ from the sample of all gluon (quark) jets in the symmetric configuration (i.e., from the $\mathrm{M}$ sample) to the value $A_{g(q)}^{\text {Bias }}$ measured from correctly identified gluon (quark) jets from the tagged samples. Only in the properties of the 
jets in the $T$ sample a small bias, typically less than $2 \%$, was found. In the case of the quark and gluons in the $\mathrm{B}$ and $\mathrm{C}$ samples the bias was determined to be zero within the Monte Carlo statistics.

The unfolded results were finally corrected for detector effects. These were estimated by comparing the properties of quark and gluon jets generated by the JETSET model before and after detector simulation. These corrections carried statistical and systematic (model dependent) errors. The latter were estimated by comparing the detector correction factors extracted from the JETSET and HERWIG models.

\subsubsection{Measured Quark and Gluon Jet Properties}

The results discussed below are presented with statistical and systematic errors. The systematic errors include contributions from the evaluation of the gluon purity, the flavour composition of the various samples, the tagging bias and the detector corrections. The dominant uncertainties, $2-5 \%$ in the central region of the distributions, come from the latter two.

The raw charged particle multiplicities measured for the jets in the respective samples are $\left\langle n^{T}\right\rangle=8.230 \pm 0.069,\left\langle n^{M}\right\rangle=7.686 \pm 0.015,\left\langle n^{B}\right\rangle=8.042 \pm 0.101$ and $\left\langle n^{C}\right\rangle=7.842 \pm 0.558$. The unfolded values, corrected for bias and detector effects are: $\left\langle n_{\text {gluon }}\right\rangle=9.90 \pm 0.10$ (stat) \pm 0.27 (syst), $\left\langle n_{u d s}\right\rangle=7.90 \pm 0.44$ (stat) \pm 0.26 (syst), $\left\langle n_{b}\right\rangle=9.32 \pm 0.27$ (stat) \pm 0.27 (syst) and $\left\langle n_{c}\right\rangle=8.37 \pm 1.64$ (stat) \pm 0.28 (syst). The difference between $b$ and light-quark jet multiplicities is consistent with the result of ref. [153]. For the natural flavour mix at the $Z$ one finds $\left\langle n^{N F M}\right.$ (quark) $\rangle=8.286 \pm 0.09$ (stat) \pm 0.22 (syst). The ratios $R_{g / q}$ between gluon and quark multiplicities are obtained as

$$
\begin{aligned}
R_{g / N F M} & =1.194 \pm 0.027(\text { stat }) \pm 0.019(\text { syst }) \\
R_{g / u d s} & =1.249 \pm 0.084(\text { stat }) \pm 0.022(\text { syst }) \\
R_{g / b} & =1.060 \pm 0.041(\text { stat }) \pm 0.020(\text { syst }) \\
R_{g / c} & =1.183 \pm 0.221(\text { stat }) \pm 0.021(\text { syst })
\end{aligned}
$$

The result $R_{g / N F M}$ is significantly larger than unity; it agrees with other LEP results [147], confirming the higher charged particle multiplicity of gluon jets. It is significantly lower than the naive asymptotic prediction of $C_{A} / C_{F}=9 / 4$. The JETSET Monte Carlo predicts $\left\langle n_{\text {gluon }}\right\rangle=$ 10.16 and $\left\langle n_{N F M}\right\rangle=7.92$ leading to a ratio of $R_{g / N F M}=1.28$ (with negligible statistical errors). The same analysis with the HERWIG Monte Carlo model yields $\left\langle n_{\text {gluon }}\right\rangle=9.48,\left\langle n_{N F M}\right\rangle=7.63$ and $R_{g / N F M}=1.24$. Finally the multiplicity ratio was determined at the parton-level of the JETSET Monte Carlo. The result, $R_{\text {part }}=1.29$, is again very similar to the hadron-level result, suggesting that the observed difference between quark and gluon jets does have a perturbative origin.

The measurement of $R_{g / b}$ indicates that for the energy scales involved, the additional particle multiplicity arising from the $B$ hadron decay masks the difference between $b$ quark and gluon jet multiplicity. This effect is present in JETSET and HERWIG which give $R_{g / b}=1.077$ and $R_{g / b}=1.003$ respectively. These measurements are also in agreement with recent results given in [154]. The unfolded ratio of gluon to light quark multiplicity is hence greater than the corresponding gluon/NFM quark ratio. The prediction for $R_{g / u d s}$ from JETSET and HERWIG are $R_{g / u d s}=1.377$ and $R_{g / u d s}=1.344$ respectively, i.e somewhat higher but within the errors of that measured. The large statistical errors do not allow firm conclusions about the gluon to $c$ quark multiplicity ratio. 
Figure 55 shows the corrected fragmentation function, $(1 / N) d N / d x_{E}$, with $x_{E}=$ $E_{\text {particle }} / E_{\text {jet }}$, for charged particles together with the estimates of the JETSET and HERWIG Monte Carlo models. Gluon jets have more particles carrying small fractions of the total energy, i.e. they are softer.

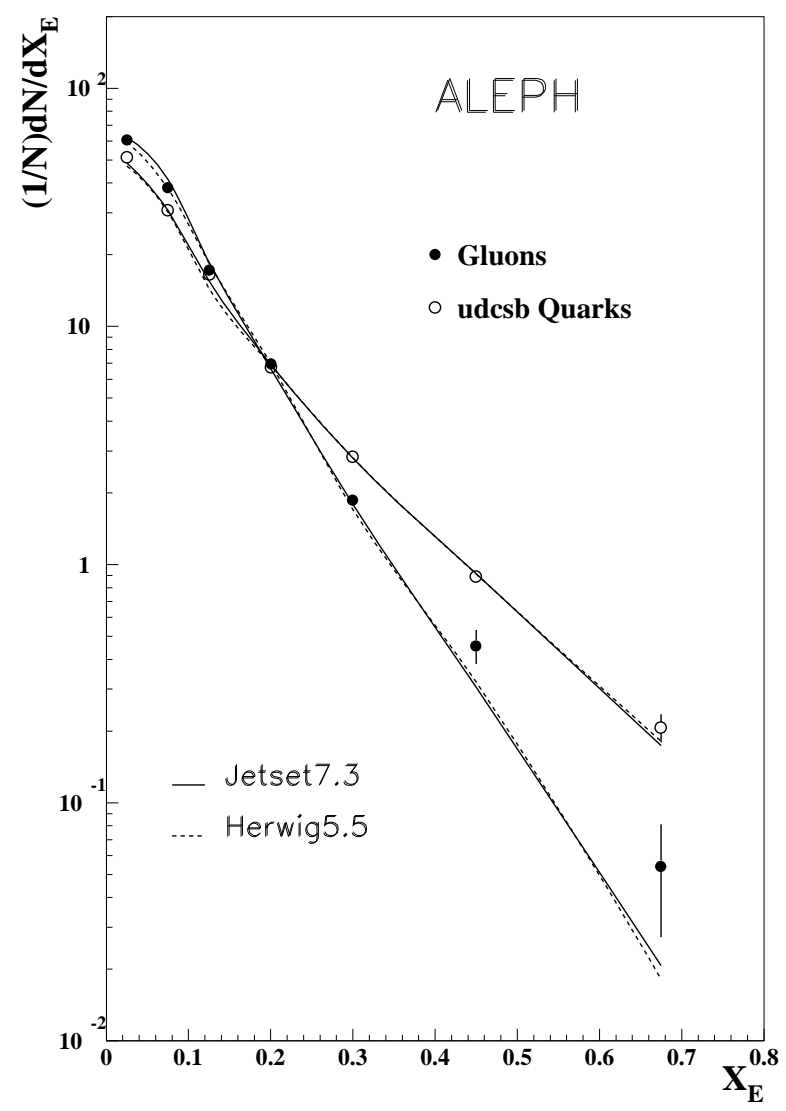

\begin{tabular}{|c|c|c|}
\hline$x_{E}$ & Quarks & Gluons \\
\hline $0.00-0.05$ & $51.13 \pm 1.18$ & $60.83 \pm 1.92$ \\
\hline $0.05-0.10$ & $30.88 \pm 1.07$ & $38.17 \pm 1.02$ \\
\hline $0.10-0.15$ & $16.50 \pm 0.65$ & $17.24 \pm 0.62$ \\
\hline $0.15-0.25$ & $6.75 \pm 0.24$ & $6.93 \pm 0.26$ \\
\hline $0.25-0.35$ & $2.85 \pm 0.13$ & $1.87 \pm 0.10$ \\
\hline $0.35-0.55$ & $0.89 \pm 0.05$ & $0.46 \pm 0.07$ \\
\hline $0.55-0.80$ & $0.20 \pm 0.03$ & $0.05 \pm 0.03$ \\
\hline
\end{tabular}

Figure 55: Fragmentation function for natural flavour mix quark and gluon jets. The numerical values of the measured cross sections are given in the associated table.

The rapidity distribution with respect to the jet axis, measured for charged particles using the pion mass, is another way of looking at the multiplicity and shape of a jet. Gluon jets, having greater multiplicity, are expected to have a higher plateau; the ratio of the heights of the corresponding distributions of gluon and quark jets is expected to asymptotically tend to $C_{A} / C_{F}$. Moreover, coherence effects, in conjunction with the selection of the events as three-jet events according to a specific jet algorithm, are expected to suppress the length of the gluon plateau [142], yielding a narrower rapidity distribution. Figure 56 shows the measurements of the rapidity distributions of the two types of jets, normalized to the total number of jets analyzed, qualitatively confirming the theoretical predictions. The heights of the quark and gluon distributions were estimated by fitting the relevant distributions with double gaussians. The ratio of the heights of the gluon and quark rapidity plateaus is measured to be $1.45 \pm 0.15$, i.e. higher than the corresponding multiplicity ratio. These measurements are in qualitative agreement with Monte Carlo predictions from the JETSET and HERWIG models, as can be seen from Fig. 56. Also shown is a comparison of the gluon rapidity distribution with the corresponding property for $b$ jets. 



Figure 56: Rapidity distributions for natural flavour mix quark and gluon jets (left) and for $b$ quark and gluon jets (right).

Another way of illustrating the broadness of a jet is to study the number of particles and the fraction of energy found within a cone around the jet axis. Figure 57 shows the integrated charged particle multiplicity and the integrated energy fraction contained within successively larger cones around the jet axis for NFM quark and gluon jets together with the corresponding estimates of JETSET and HERWIG. Gluon jets are clearly broader. Although gluon jets have higher total multiplicity, quark jets contain more particles within a cone of half angle up to $\approx 15^{\circ}$. Quark jets have on average about $32 \%$ of their energy enclosed within a half cone of $5^{\circ}$, compared to only $16 \%$ for gluon jets. In general the models reproduce the data well.

\subsection{Prompt Photon Production}

\subsubsection{Isolated Photon Studies}

Several studies have been made of the production of hard isolated photons accompanying hadronic decays of the Z at LEP [155]. The origin of these photons has been attributed to final state radiation (FSR), emitted at an early stage in the QCD parton evolution process, from the primary quark-antiquark pair. Hard photons are the only partons produced close to the primary interaction that are observed free from fragmentation effects. The study of FSR photons thus gives a unique insight into the parton evolution mechanism. Since photons are produced in the parton shower in the same way as gluons, measurements of hard photon production may lead to a better understanding of the quark-gluon showering process. The main approach of this earlier work has been to test the detailed predictions of the parton shower models, JETSET [25], HERWIG [31] and ARIADNE [30], and to compare the data with QCD $\mathcal{O}\left(\alpha \alpha_{\mathcal{S}}\right)$ calculations at the parton level [156, 157]

In all of these analyses, the candidate photon was isolated from the hadronic debris in an event using a geometrical cone centred around its direction inside of which a minimal residue of accompanying hadronic energy was allowed. This procedure was considered necessary in order to reduce the non-prompt photon backgrounds from hadron decays. The photon then was removed from the event before jets were formed with the other particles using the JADE clustering algorithm. As a consequence, any particles associated with the photon were incorporated into the other jets. Finally, a photon was retained only if the restored candidate photon remained apart from the jets in a second application of the clustering algorithm. In this 

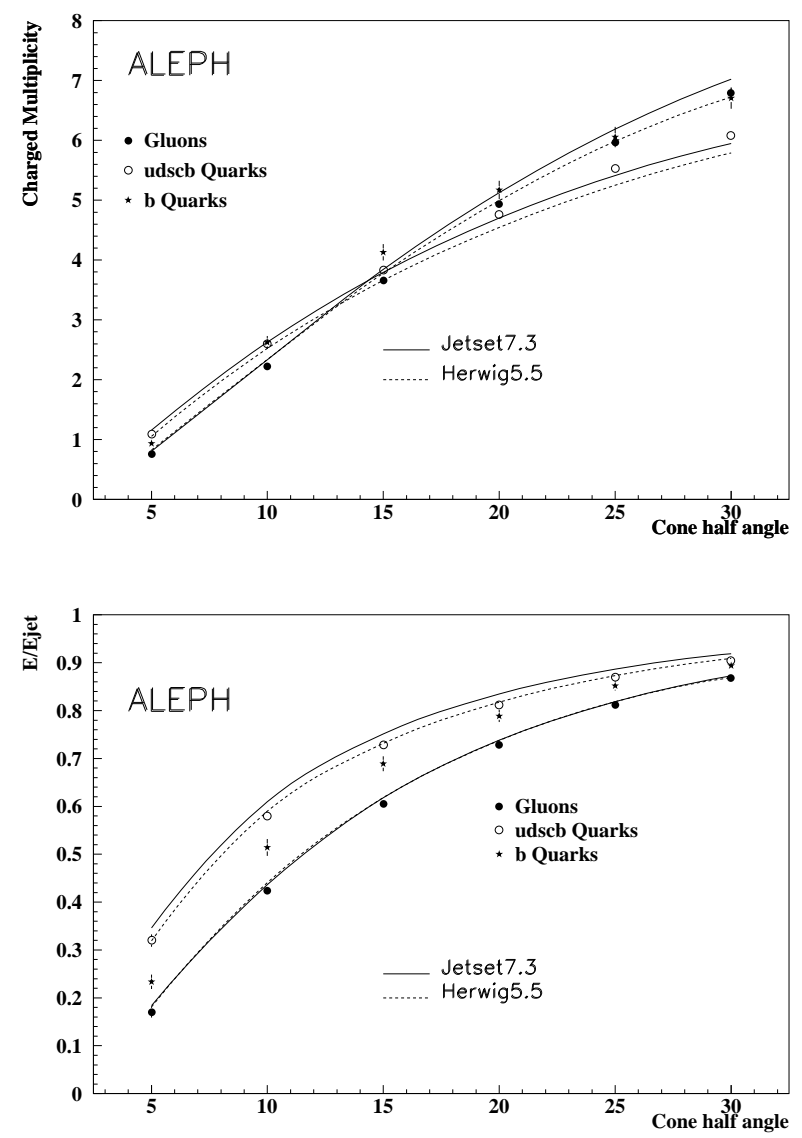

Figure 57: Integrated charged multiplicity within successive cones for quark and gluon jets (upper plot) and integrated energy fraction within successive cones for quark and gluon jets (lower plot).

latter procedure, the jet resolution parameter $y_{c u t}$ was defined in terms of the opening angle between jet $i$ and the photon candidate. It thus provides a convenient measure of the degree of isolation. However, the method for isolating photons by the use of a geometrical cone is sensitive to the distribution of low energy fragments around jets.

The early measurements using the isolation cone procedure revealed large discrepancies with the available QCD predictions. A detailed comparison of the measured FSR rate with parton shower models as a function of $y_{c u t}$ (Fig. 58) showed that the models considered (JETSET, ARIADNE and HERWIG) reproduced the general shape of the distribution, indicating that FSR is responsible for the observed events, but could not agree with the absolute FSR rate. The JETSET prediction is three standard deviations too low at low $y_{\text {cut }}$ whereas the ARIADNE prediction is two to three standard deviations higher than the data at high $y_{\text {cut }}$. The HERWIG prediction is within one or two standard deviations of the data. All three parton shower models were shown to have difficulties in describing the $\gamma+n$-jet rates as a function of $y_{c u t}, n$ being the number of jets produced in addition to the isolated photon.

Comparisons of the data with matrix element calculations, as implemented in the Monte Carlo programs GS [156] and GNJETS [157], reproduced the data only at high $y_{c u t}$. The predictions for the total FSR rate and for the $\gamma+n$-jet rate were sensitive to the values of cutoff parameters in the calculations. Except at high $y_{c u t}$, the GS prediction differs significantly from that of GNJETS. The various QCD calculations required large $\alpha_{s}$-dependent corrections 


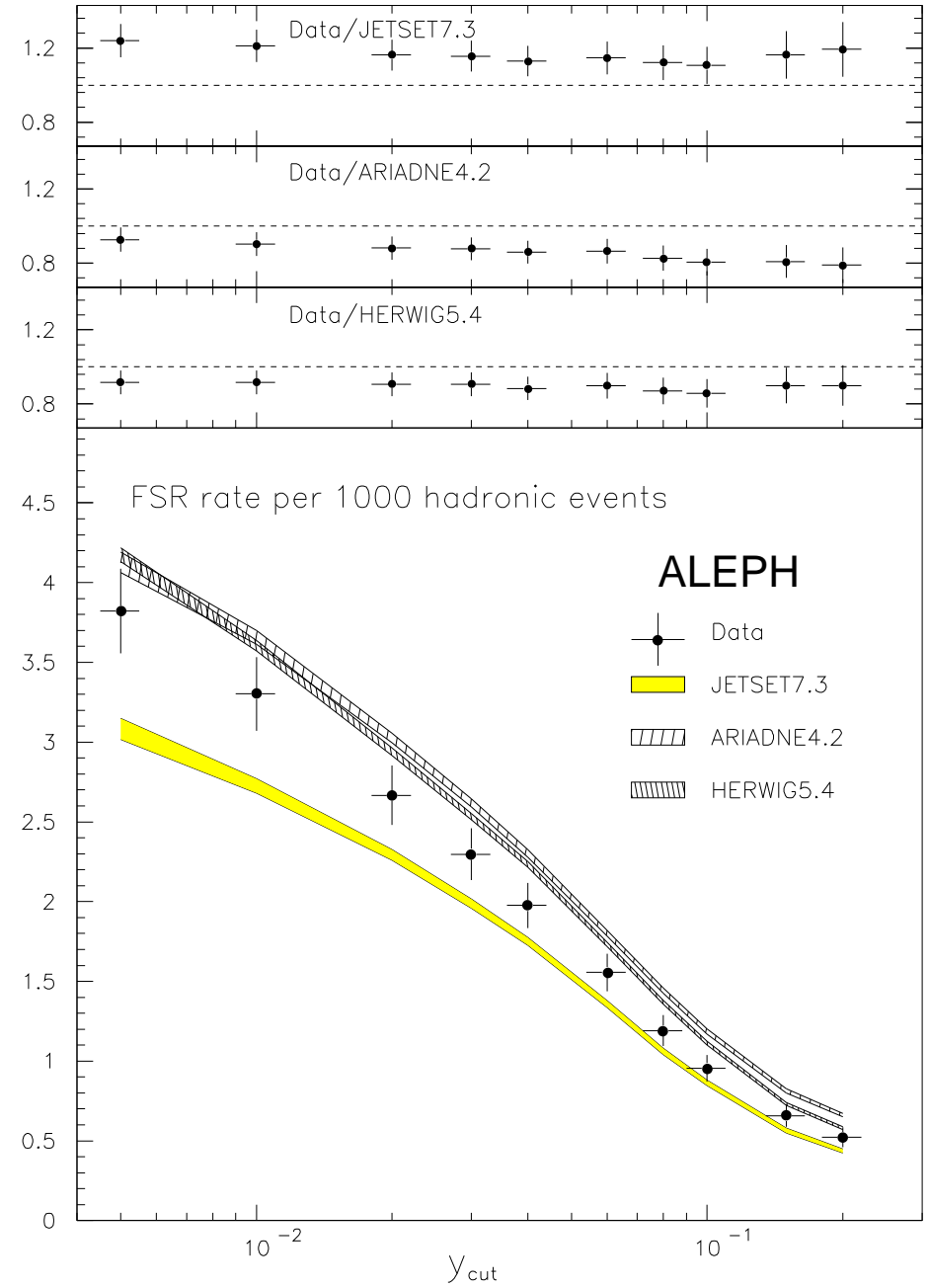

Figure 58: The acceptance corrected FSR rate as a function of $y_{c u t}$ and its comparison with parton shower models. The band around each prediction corresponds to the statistical and theoretical errors added in quadrature.

to approach the data.

\subsection{2 "Democratic" Analysis}

It was pointed out [156] that a safer approach would be to apply a jet recombination scheme simultaneously to all particles in an event, including the photon. This "democratic" approach enables the phase space regions for all event topologies to be properly defined and handles correctly those hadrons which are associated naturally with the photon. However, it introduces a significant non-perturbative contribution to the cross section which depends upon the amount of accompanying energy allowed in the "photon jet". At first sight, this would appear to prevent the accurate comparison of data with the QCD predictions employed earlier. However, a significant part of the parton-to-photon fragmentation function can be measured, allowing this non-perturbative contribution to be determined. This adds new information to the dynamics of quark radiation, and at the same time improves the comparison of all FSR data with the QCD calculations.

The fractional energy of a photon within a jet can be defined as $z_{\gamma}=E_{\gamma} /\left(E_{\gamma}+E_{\text {had }}\right)$, where $E_{\text {had }}$ is the energy of all the accompanying hadrons in the "photon-jet" found by the clustering algorithm. Recently, it has been shown in an $\mathcal{O}\left(\alpha \alpha_{f}\right)$ QCD calculation by Glover and Morgan [158] that the perturbative contributions to the quark to photon fragmentation 
function, $D\left(z_{\gamma}\right)$, can be evaluated, thus allowing the non-perturbative part of the $D\left(z_{\gamma}\right)$ function to be determined from the data. At the $\mathrm{Z}$, the measured value of $D\left(z_{\gamma}\right)$ is the average of the $D\left(z_{\gamma}\right)$ functions for the combination of the two $u$-type and three $d$-type quark flavours weighted by their respective electro-weak couplings and electric charges. Thus, $D\left(z_{\gamma}\right)$ can be obtained from the normalized differential two-jet cross section:

$$
\frac{1}{\sigma_{h a d}} \frac{d \sigma(2-\mathrm{jet})}{d z_{\gamma}}=D\left(z_{\gamma}\right) G_{L E P}
$$

where $G_{L E P}$ is twice the ratio of the FSR correction to the total hadronic cross section at the Z. From currently measured values $[159] G_{L E P}=2.51 \times 10^{-4}$. This normalization does not take into account any other source of flavour dependence in the fragmentation function.

\section{Selection of Events with Final State Photons}

For the "democratic analysis" the hadronic Z decays were selected using standard procedures described in Section 1.3. The overall efficiency of the photon selection is $55 \pm 2.4 \%$ and is almost independent of the energy of the photon. The contribution from non-prompt photons, mainly $\pi^{0}$ 's, is determined from Monte Carlo. The accuracy of this simulation was studied in [160]. For each event with at least one selected photon, jets were constructed using all the energy flow objects of the event and treating the photon equally with all the other particles. The particle clustering was performed using the Durham algorithm [28]. An event was kept if at least one jet contained a selected photon with $z_{\gamma}>0.7$. This procedure was repeated successively for thirteen different $y_{c u t}$ values increasing from 0.001 to 0.33 . For each value of $y_{c u t}$, the event sample then was divided into three categories corresponding to jet topologies of two, three and $\geq 4$ jets, where the number of jets includes the photon jet.

Since the non-perturbative part of $D\left(z_{\gamma}\right)$ is naturally associated with the hadronization process, the measured jet rates are not corrected back to the parton level.

\section{Analysis of Two-Jet Events}

At the primary parton level, two-jet topologies correspond to $q \bar{q} \gamma$ events where either the $q$ and $\bar{q}$ coalesce to form one jet or one of the quarks radiates (or fragments into) a photon which remains part of the quark jet. In the absence of radiated gluons, the first case leads to completely isolated photons with $z_{\gamma}=1$ whereas the second populates the full $z_{\gamma}$ distribution. Thus, it is expected that the quark-to-photon fragmentation function, $D\left(z_{\gamma}\right)$, will decrease monotonically towards $z_{\gamma}=1$ where the isolated component becomes the principal contribution.

Figure 59 shows the corrected differential $z_{\gamma}$ distributions normalized to the total hadronic event sample for four values of $y_{c u t}$. A downward trend is observed up to $z_{\gamma}=0.95$, and the isolated photon peak in the final bin $0.99<z_{\gamma}<1.0$ is clearly evident. However, it appears that a fraction of this isolated component populates the $0.95<z_{\gamma}<0.99$ bin. This broadening effect becomes more pronounced with increasing $y_{c u t}$. Both the ARIADNE and HERWIG parton-shower Monte Carlos ascribe this to the association of soft hadrons produced in the parton shower to the photon jet by the clustering algorithm. No such effect is observed at the parton level. This is the only portion of the $z_{\gamma}$ distribution where significant differences between hadron and parton levels appear. 

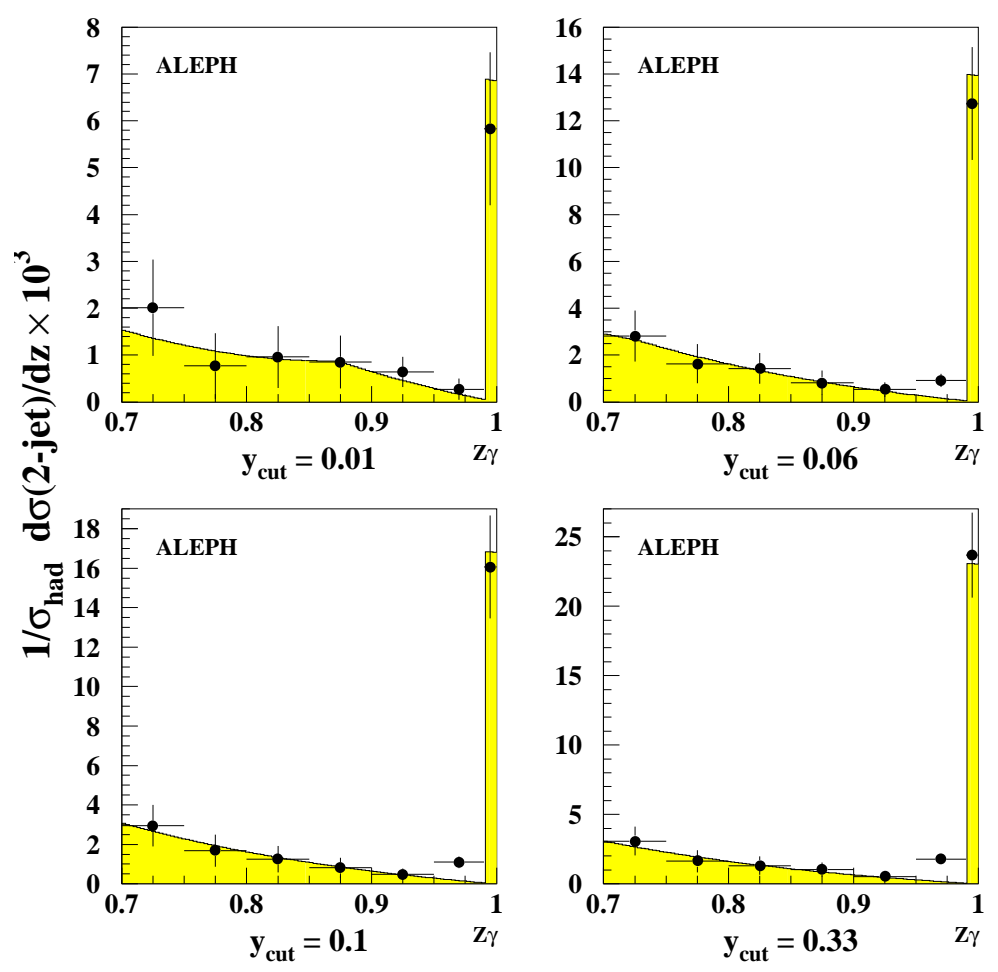

Figure 59: The two-jet rates measured for four values of $y_{\text {cut }}$. The data, shown as circular dots with error bars, is compared with a universal fragmentation function calculated using $B\left(z_{\gamma}, \mu_{0}\right)=-1-$ $\log \left(s / 2 \mu_{0}^{2}\right)$ with $\mu_{0}=0.14 \mathrm{GeV}$ (see text).

\section{Parametrization of the Non-Perturbative Component of $D\left(z_{\gamma}\right)$}

Following the leading order formalism of [158] developed in the $\overline{M S}$ renormalization scheme, the inclusive quark-to-photon fragmentation function $D\left(z_{\gamma}\right)$ can be written in the following way for large $z_{\gamma}[160]$ :

$$
D\left(z_{\gamma}\right)=\frac{1+\left(1-z_{\gamma}\right)^{2}}{z_{\gamma}} \log \left(\frac{z_{\gamma}}{1+z_{\gamma}} \frac{s}{\mu_{0}^{2}}\right)+B\left(z_{\gamma}, \mu_{0}\right)+f\left(z_{\gamma}, y_{c u t}\right)+\frac{1}{2} R_{\Delta}\left(y_{c u t}\right) \delta\left(1-z_{\gamma}\right)
$$

where $f\left(z_{\gamma}, y_{\text {cut }}\right)$ is a known regular function with $f\left(z_{\gamma}=1\right)=1$ and $R_{\Delta}$ is the perturbative component of the fragmentation function for isolated photon production without accompanying parton energy. For $z_{\gamma}>0.7, f$ becomes independent of $y_{\text {cut }}$ when $y_{\text {cut }}>0.07$, because then the photon always combines with its radiating quark to form a jet. Therefore, apart from the $R_{\Delta}$ contribution, $D\left(z_{\gamma}\right)$ is expected to be independent of $y_{c u t}$ in this region, as is observed. The free parameters to be determined are the cut-off scale $\mu_{0}$ and the function $B\left(z_{\gamma}, \mu_{0}\right)$. The two highest $z_{\gamma}$ bins are combined into one bin with $z_{\gamma}>0.95$ to take into account the observed broadening of the isolated component. It is then assumed that the isolated component in the data is concentrated entirely in this bin. Since the magnitude and $z_{\gamma}$ dependence of $B\left(z_{\gamma}, \mu_{0}\right)$ are unknown, various parametrizations have been tried in fitting $D\left(z_{\gamma}\right)$ to the six data points in the range $0.7<z_{\gamma}<1.0$. An adequate representation of the data is obtained with $B\left(z_{\gamma}, \mu_{0}\right)=C$, where $C$ is a constant.

When a two-parameter fit is made to the data the values of $C$ and $\mu_{0}$ are found to be strongly correlated. This is related to the observation (Fig. 59) that the fragmentation function approaches zero at $z_{\gamma}=1$ when the isolated component, $R_{\Delta}$, is disregarded. In fact, imposing 
the condition $D\left(z_{\gamma}=1\right)=R_{\Delta} / 2$ yields the following relation between $C$ and $\mu_{0}$ :

$$
C=-1-\log \left(\frac{s}{2 \mu_{0}^{2}}\right) .
$$

The fit results are found to be in very good agreement with this simple relation. A more precise value of $\mu_{0}$ can be obtained by performing a single parameter fit to the data using the parametrization of $B\left(z_{\gamma}, \mu_{0}\right)=C$ where the latter is constrained by the above relation. The best value is found to be:

$$
\mu_{0}=0.14_{-0.08-0.04}^{+0.21+0.22} \mathrm{GeV} \quad \text { with } \quad \chi^{2} / 5=0.37
$$

The single parameter fit was repeated for $y_{\text {cut }}$ values of $0.008,0.02,0.1$ and 0.33 and consistent results are found showing that over this range, the non-perturbative term is universal, as expected, and any $y_{c u t}$ dependence in the perturbative parts, including the isolated component, are adequately described by the leading order calculations.

Isolated Photon Region: $0.95<z_{\gamma}<1$

The integrated rates above $z_{\gamma}=0.95$ are now compared with $D\left(z_{\gamma}\right)$ described by Eq. (83) where the fitted value of $\mu_{0}=0.14 \mathrm{GeV}$ and the corresponding value for $C$ are substituted. Figure 60 shows the result of this comparison as a function of $y_{c u t}$. The agreement is adequate over the full range of $y_{c u t}$. It should be noted that the predictions of this leading order formalism for the two-jet rate contain perturbative components which are derived from a pure QED calculation. In previous two-step analyses [155], a large $\alpha_{s}$-dependent next-to-leading order QCD correction was needed to describe the two-jet rate for isolated photons.

Figure 60 also shows that JETSET falls substantially below the data at all values of $y_{\text {cut }}$ in contrast to ARIADNE and HERWIG (not shown) where the agreement with data is satisfactory at high $y_{\text {cut }}$.

\section{Three and Four-Jet Event Rates}

It is of interest to see if a good description of the other dominant jet rates can be obtained using the same formalism. The $z_{\gamma}$ distributions for the three- and $\geq 4$-jet events are quite different from the two-jet topologies, being dominated by the isolated photon peak near $z_{\gamma}=1$. The acceptance corrected $z_{\gamma}$ distributions are compared at each value of $y_{c u t}$ with the same $\mathcal{O}\left(\alpha \alpha_{s}\right)$ calculation [158] which now includes the non-perturbative part of $D\left(z_{\gamma}\right)$ measured from the two-jet rate. This is implemented in an updated version of the matrix element program EEPRAD [156]. The only free parameter is $\alpha_{s}$. To compare with the predictions of EEPRAD, the rates are integrated above $z_{\gamma}=0.95$ with the non-perturbative parts of the fragmentation function included. Figure 61 shows the results of this comparison. Effectively, the value of $\alpha_{s}=0.17$ compensates for the missing higher orders and other scheme-dependent factors neglected in EEPRAD. Both the predicted three-jet and $\geq 4$-jet rates follow the data closely down to very low values of $y_{\text {cut }} \sim 0.003$.

It should be noted, however, that this choice of $\alpha_{s}$ leads to a good description of the data only above $z_{\gamma}=0.95$ where the isolated component dominates. In the lower $z_{\gamma}$ fragmentation region this leading order description is inadequate. 

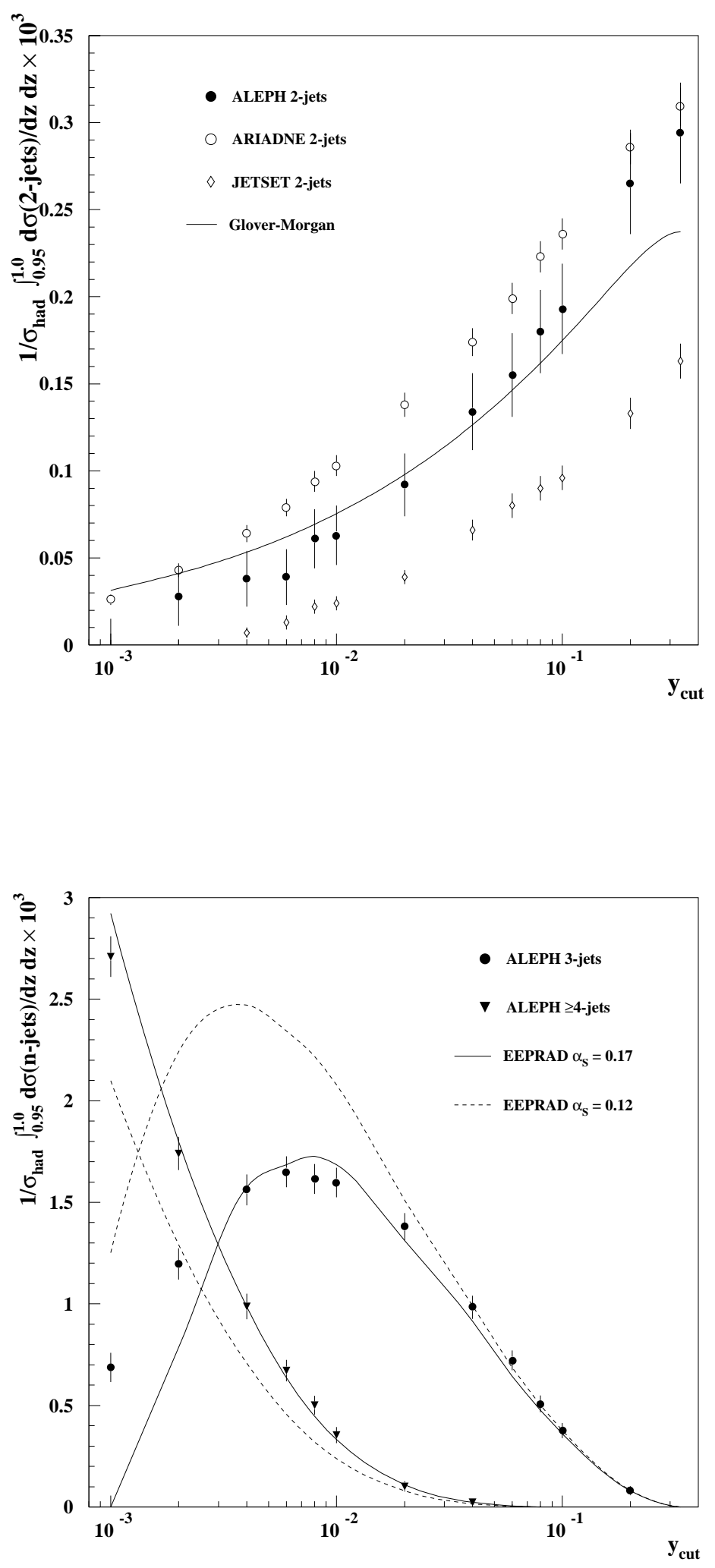

Figure 60: Integrated two-jet rate above $z_{\gamma}=0.95$ as function of $y_{\text {cut }}$, compared with ARIADNE, JETSET and the full QCD calculation including the fitted non-perturbative component of the $D\left(z_{\gamma}\right)$ function.
Figure 61: The three-jet and $\geq$ fourjet integrated rates with $z_{\gamma} \geq 0.95$ as a function of $y_{c u t}$, compared with EEPRAD predictions.

In conclusion, the measured quark-to photon fragmentation function can be described by a factorization-scale-independent QCD leading order prescription with non-perturbative contributions in which the only free parameter is a cut-off mass scale which can be determined 
from the data. A satisfactory description of all aspects of the measured two-jet rates then can be found. The other dominant jet rates can be described using the same formalism with a large value of $\alpha_{s}$ to take into account uncalculated higher orders. 


\section{Hadronization}

A measurement of the composition of the hadronic final state in $\mathrm{e}^{+} \mathrm{e}^{-}$annihilation is fundamental to an understanding of the fragmentation of quarks and gluons into hadrons. While no calculable theory yet exists for this process, a number of phenomenological models have evolved, falling into two broad classes: "string" fragmentation and "cluster" fragmentation, as exemplified by the JETSET [25] and HERWIG [31] Monte Carlos, respectively. The models contain a large number of parameters controlling the spin/ flavour assignment of the produced hadrons. As already discussed in Section 2.3, these parameters have been tuned using the data presented in this section. Therefore, one can speak of "model predictions" only in a restricted sense. Nevertheless, it is important to see how well all measurements are described within the framework of a certain model.

In the model calculations three different sources for the observed hadrons can be distinguished: leading particles containing the initial quark of the $\mathrm{Z} \rightarrow q \bar{q}$ decay, direct fragmentations products and decay products of heavier particles. Since the production of heavy quarks in the fragmentation chain is strongly suppressed, hadrons containing $b$ or $c$ quarks are expected to be leading particles or decay products of them. The ALEPH results on bottom and charm production from $Z$ decay have been discussed elsewhere [35, 36, 37]. In this section experimental results on the inclusive production of identified light hadrons are presented, summing over all initial quark flavours and all sources. In the first section, 5.1, a search for free quarks is presented.

\subsection{Search for Free Quarks}

Quarks cannot exist as free particles and therefore fragment into the well known hadrons. As a prelude to the study of these hadrons in this section, the test of this fundamental property of QCD by ALEPH in the early days of LEP is reviewed briefly.

The ALEPH search for free quarks [6] was performed with the data from the years 1989 and 1990 , corresponding to about $180,000 \mathrm{Z}$ events, using the ionization measurement of charged particles in the TPC. Particles $X$ with unexpected ionization such as free quarks with fractional charge or heavy particles were searched for. At the $Z$ peak, quarks are produced with high momenta and might become free without contradicting earlier limits. The search is valid for particles which are long-lived and have an interaction length comparable to or larger than that of the known stable hadrons, so that they are observable in the TPC.

The particles $X$ for which limits were derived could appear in pairs, inclusively with jets $\left(e^{+} e^{-} \rightarrow X \bar{X}+\right.$ hadrons $)$ or exclusively. Also the case in which the particle could appear singly along with other hadronic activity and in which conservation laws would be maintained via production of a low mass partner were studied.

In the data, no candidates were found for masses above $5 \mathrm{GeV} / c^{2}$. The limit for the production of new charged particles can be expressed in terms of the dimuon cross section. The $90 \%$ C.L. limits [6] are shown in Fig. 62 for the inclusive production of charged particles with unexpected mass and charge, assuming a momentum distribution for the new particles according to $E \frac{d N}{d p^{3}}=$ const. This can be considered as a parametrization for the fragmentation function for the production of massive particles (see [6] for an alternative assumption and for further references). It is seen that these results extend the limits given by previous experiments into the mass range $15-45 \mathrm{GeV} / c^{2}$. 



Figure 62: The $90 \%$ C.L. limit for the inclusive production of charged particles with unexpected mass and charge. The area above the curves is excluded.

\subsection{Inclusive Production of Identified Hadrons}

\subsubsection{Identified Stable Charged Particles}

Inclusive $\pi^{ \pm}, \mathrm{K}^{ \pm}$and $(\mathrm{p}, \overline{\mathrm{p}})$ differential cross sections in hadronic decays of the $\mathrm{Z}$ have been measured as a function of $x_{p}=p_{\text {hadron }} / p_{\text {beam }}$, the scaled momentum. Charged particles are identified by their rate of ionization energy loss in the ALEPH Time Projection Chamber. The ionization deposited by a track traversing the entire TPC is sampled on up to 338 sense wires. The specific energy loss is estimated from the truncated mean of the usable samples, discarding the lower $8 \%$ and upper $40 \%$. For a minimum ionizing track with 270 samples and a mean sample length of $0.5 \mathrm{~cm}$, the $d E / d x$ resolution is $7 \%$. The differential cross sections were determined by a maximum-likelihood fit to the measured $d E / d x$ distribution in bins of $x_{p}$. The $2 \%-5 \%$ contamination in the pion rate from muons, which were not distinguished in the fit, was corrected according to the prediction of JETSET. The typical acceptance for tracks was $50 \%$ for all species, dipping to $35 \%$ at high momentum due to overlaps.

The results are based on approximately 520000 events measured by ALEPH in 1992. Details of the analysis are described in ref. [161].

In Figure 63 the differential cross sections

$$
\frac{1}{\sigma_{t o t}} \frac{d \sigma}{d x_{p}}(\mathrm{Z} \rightarrow h+X)
$$

are shown for $h=\pi^{ \pm}, \mathrm{K}^{ \pm}$and $(\mathrm{p}, \overline{\mathrm{p}})$. Here $\sigma_{\text {tot }}$ is the total cross section for the process $\mathrm{Z} \rightarrow$ hadrons. The ALEPH results are compared to the predictions of the JETSET 7.4, HERWIG 5.8 and ARIADNE 4.08 Monte Carlos.

Except for very small momenta there is reasonable agreement in the $\pi^{ \pm}$differential cross section. All models predict a softer $\mathrm{K}^{ \pm}$spectrum than is observed. The proton spectrum 

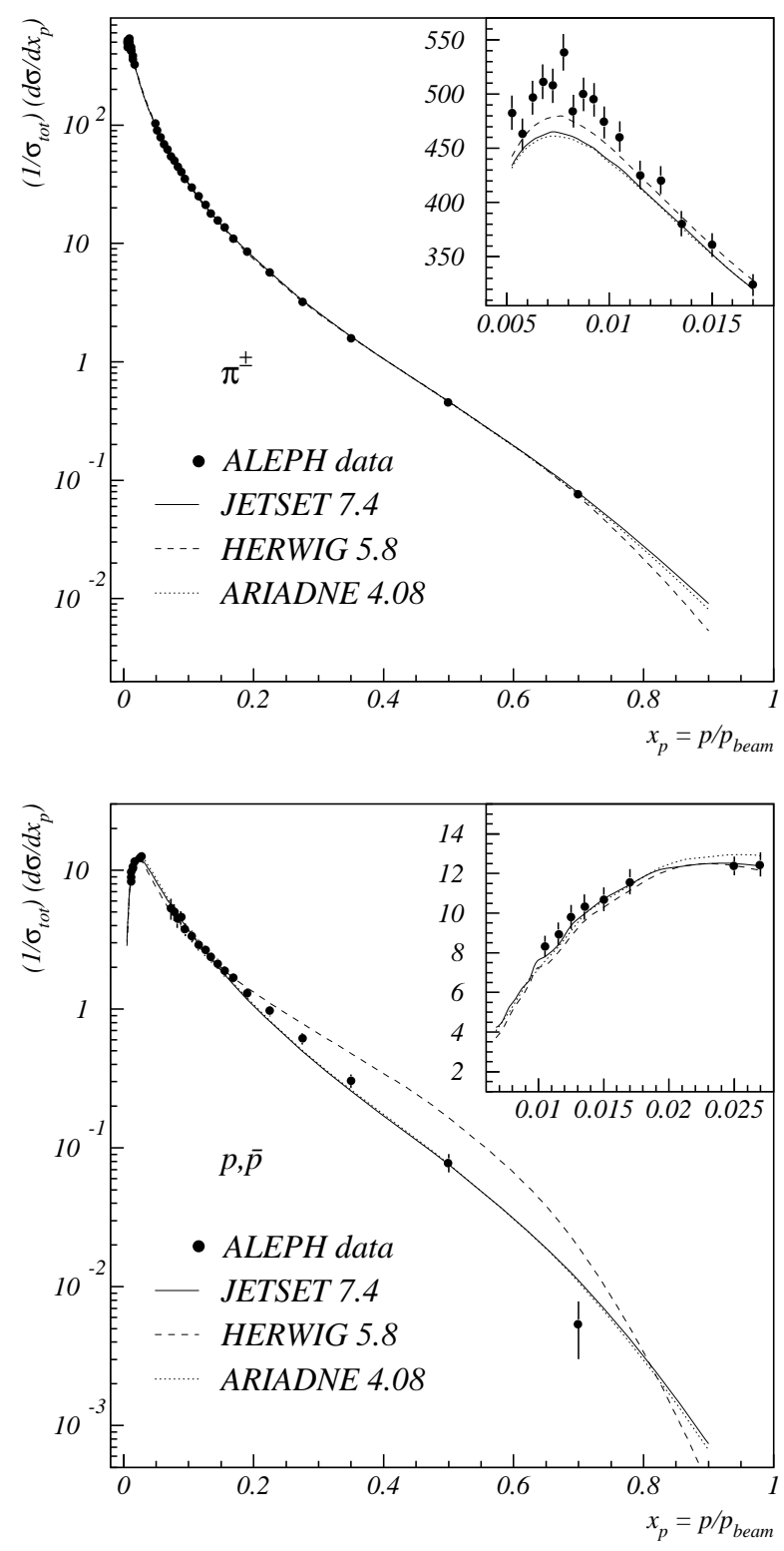

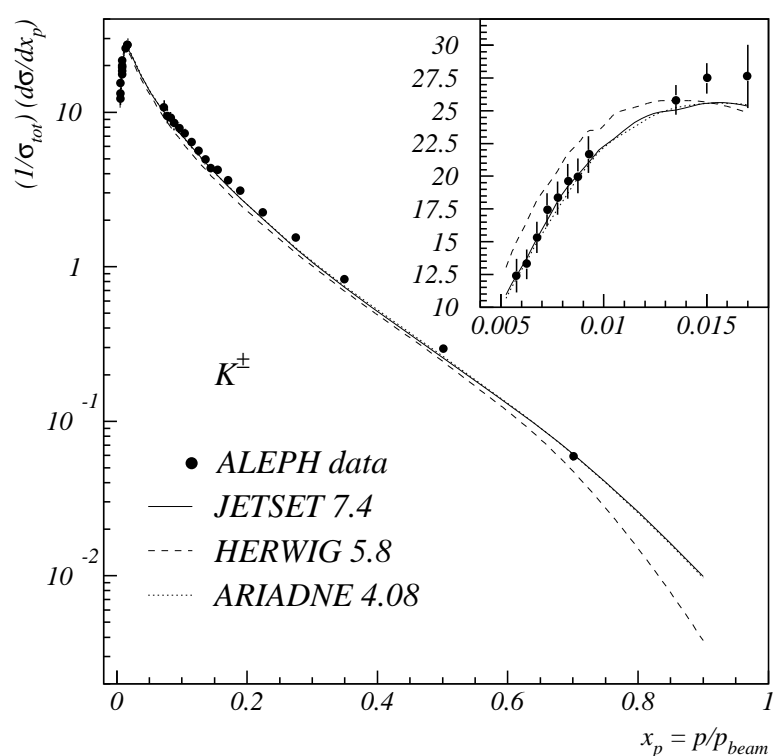

Figure 63: Differential cross section as a function of $x_{p}=p_{\text {hadron }} / p_{\text {beam }}$ for $\pi^{ \pm}, \mathrm{K}^{ \pm}$ and $(p, \bar{p})$ compared with the predictions of JETSET, HERWIG and ARIADNE. The errors shown are the quadratic sum of statistical and systematic errors.

at high momenta is underestimated by JETSET and ARIADNE. In HERWIG it is grossly overestimated. The experimental results are listed in Table 28. The individual contributions to the overall error are shown separately.

In Fig. 64 the ratios of the rates of kaons to pions and protons to pions are shown as a function of $x_{p}$, together with the Monte Carlo predictions. In the ratios most systematic errors cancel. With the parameter values as determined by ALEPH, the ratio of strange to non-strange mesons above $x_{p}=0.2$ is underestimated by all three models, and none of them reproduces the fraction of protons as a function of $x_{p}$, even after enabling the mechanism for leading baryon suppression in JETSET (Section 2.3).

An important property of perturbative QCD is the coherence of gluon radiation. As already discussed in Section 4.1, destructive interference reduces the phase space for soft gluon emission leading to a suppression of gluons at low $x_{p}$. The $\xi=\ln \left(1 / x_{p}\right)$ distribution for gluons can be calculated in the modified leading logarithm approximation (MLLA), in which dominant leading and next-to-leading order terms at each branching are resummed to all orders. This is equivalent to a parton shower including coherence. The distribution is asymptotically Gaussian 


\begin{tabular}{|c|c|c|c|c|c|c|c|c|c|c|}
\hline & & & & & & $\frac{1}{\sigma_{t o t}} \frac{d \sigma}{d x_{1}}$ & & & & \\
\hline$x_{p}$ interval & $\left\langle x_{p}\right\rangle$ & & $\pi^{ \pm}$ & & & $\mathrm{K}^{ \pm}$ & & & $(\mathrm{p}, \overline{\mathrm{p}})$ & \\
\hline $0.0050-0.0055$ & 0.00526 & 482.9 & \pm 5.9 & \pm 1.3 & & & & & & \\
\hline $0.0055-0.0060$ & 0.00574 & 462.6 & \pm 4.8 & \pm 0.9 & $12.40=$ & \pm 1.12 & \pm 0.01 & & & \\
\hline $0.0060-0.0065$ & 0.00622 & 496.5 & \pm 4.6 & \pm 0.8 & $13.27=$ & \pm 0.91 & & & & \\
\hline $0.0065-0.0070$ & 0.00673 & 511.2 & \pm 4.4 & \pm 0.8 & $15.33=$ & \pm 0.90 & \pm 0 & & & \\
\hline $0.0070-0.0075$ & 0.00722 & 507.7 & \pm 4.2 & \pm 0.7 & $17.43=$ & \pm 0.92 & \pm 0.02 & & & \\
\hline $0.0075-0.0080$ & 0.00773 & 538.5 & \pm 4.4 & \pm 0.7 & $18.33=$ & \pm 0.88 & \pm 0 & & & \\
\hline $0.0080-0.0085$ & 0.00822 & 484.2 & \pm 3.9 & \pm 0.6 & $19.62=$ & \pm 0.90 & \pm 0 & & & \\
\hline $0.0085-0.0090$ & 0.00871 & 499.7 & \pm 3.9 & \pm 0.7 & $20.02=$ & \pm 0.86 & \pm 0.05 & & & \\
\hline $0.0090-0.0095$ & 0.00922 & 494.6 & \pm 3.8 & \pm 0.6 & $21.66=$ & \pm 0.88 & \pm 0.12 & & & \\
\hline $0.0095-0.010$ & 0.00972 & 473.9 & \pm 3.6 & \pm 0.5 & & & & & & \\
\hline $0.010-0.011$ & 0.0105 & 460.9 & \pm 2.5 & \pm 0.5 & & & & 8.32 & \pm 0.35 & \pm 0.00 \\
\hline $0.011-0.012$ & 0.0115 & 425.6 & \pm 2.3 & \pm 0.5 & & & & 8.95 & \pm 0.36 & \pm 0.00 \\
\hline $0.012-0.013$ & 0.0125 & 420.7 & \pm 2.3 & \pm 0.4 & & & & 9.80 & \pm 0.36 & \pm 0.01 \\
\hline $0.013-0.014$ & 0.0135 & 380.5 & \pm 2.2 & \pm 0.4 & $25.84=$ & \pm 0.66 & \pm 0.50 & 10.30 & \pm 0.38 & \pm 0.01 \\
\hline 0.014-0.016 & 0.0147 & 360.8 & \pm 1.5 & \pm 0.6 & $27.46=$ & \pm 0.47 & \pm 0.68 & 10.70 & \pm 0.26 & \pm 0.01 \\
\hline $0.016-0.018$ & 0.0167 & 324.0 & \pm 1.4 & \pm 1.8 & $27.63=$ & \pm 0.53 & \pm 2.20 & 11.58 & \pm 0.27 & \pm 0.04 \\
\hline $0.024-0.026$ & 0.0247 & & & & & & & 12.37 & \pm 0.18 & \pm 0.23 \\
\hline $0.026-0.028$ & 0.0268 & & & & & & & 12.46 & \pm 0.18 & \pm 0.44 \\
\hline $0.045-0.050$ & 0.0470 & 103.96 & \pm 0.61 & \pm 2.09 & & & & & & \\
\hline $0.050-0.055$ & 0.0520 & 89.95 & \pm 0.53 & \pm 1.02 & & & & & & \\
\hline $0.055-0.060$ & 0.0570 & 78.96 & \pm 0.50 & \pm 0.90 & & & & & & \\
\hline $0.060-0.065$ & 0.0619 & 69.36 & \pm 0.35 & \pm 0.72 & & & & & & \\
\hline $0.065-0.070$ & 0.0669 & 61.35 & \pm 0.33 & \pm 0.60 & & & & & & \\
\hline $0.070-0.075$ & 0.0719 & 55.27 & \pm 0.32 & \pm 0.49 & \begin{tabular}{|l|}
10.60 \\
\end{tabular} & \pm 0.30 & \pm 1.28 & 5.315 & \pm 0.216 & \pm 0.876 \\
\hline $0.075-0.080$ & 0.0769 & 49.91 & \pm 0.30 & \pm 0.44 & 9.53 & \pm 0.26 & \pm 0.98 & 5.008 & \pm 0.183 & \pm 0.639 \\
\hline $0.080-0.085$ & 0.0819 & 44.33 & \pm 0.29 & \pm 0.38 & 9.15 & \pm 0.23 & \pm 0.83 & 4.445 & \pm 0.162 & \pm 0.549 \\
\hline $0.085-0.090$ & 0.0870 & 40.24 & \pm 0.27 & \pm 0.34 & 8.41 & \pm 0.21 & \pm 0.71 & 4.555 & \pm 0.154 & \pm 0.474 \\
\hline $0.090-0.10$ & 0.0942 & 35.38 & \pm 0.18 & \pm 0.30 & 7.96 & \pm 0.14 & \pm 0.56 & 3.742 & \pm 0.092 & \pm 0.355 \\
\hline $0.10-0.11$ & 0.104 & 29.51 & \pm 0.17 & \pm 0.25 & 7.26 & \pm 0.13 & \pm 0.47 & 3.355 & \pm 0.084 & \pm 0.292 \\
\hline $0.11-0.12$ & 0.114 & 24.91 & \pm 0.16 & \pm 0.22 & 6.34 & \pm 0.11 & \pm 0.37 & 2.905 & \pm 0.077 & \pm 0.232 \\
\hline $0.12-0.13$ & 0.124 & 21.06 & \pm 0.14 & \pm 0.18 & 5.63 & \pm 0.11 & \pm 0.32 & 2.653 & \pm 0.072 & \pm 0.205 \\
\hline $0.13-0.14$ & 0.134 & 18.16 & \pm 0.13 & \pm 0.16 & 4.94 & \pm 0.10 & \pm 0.28 & 2.371 & \pm 0.068 & \pm 0.178 \\
\hline $0.14-0.15$ & 0.144 & 15.46 & \pm 0.12 & \pm 0.15 & 4.39 & \pm 0.09 & \pm 0.24 & 2.137 & \pm 0.064 & \pm 0.162 \\
\hline $0.15-0.16$ & 0.154 & 13.64 & \pm 0.12 & \pm 0.13 & 4.22 & \pm 0.09 & \pm 0.22 & 1.878 & \pm 0.061 & \pm 0.146 \\
\hline $0.16-0.18$ & 0.169 & 11.00 & \pm 0.07 & \pm 0.11 & 3.63 & \pm 0.06 & \pm 0.18 & 1.696 & \pm 0.041 & \pm 0.118 \\
\hline $0.18-0.20$ & 0.189 & 8.484 & \pm 0.066 & \pm 0.094 & 3.10 & \pm 0.05 & \pm 0.15 & 1.299 & \pm 0.036 & \pm 0.099 \\
\hline $0.20-0.25$ & 0.222 & 5.621 & \pm 0.035 & \pm 0.071 & 2.245 & \pm 0.029 & \pm 0.109 & 0.966 & \pm 0.020 & \pm 0.073 \\
\hline $0.25-0.30$ & 0.272 & 3.181 & \pm 0.026 & \pm 0.047 & 1.538 & \pm 0.025 & \pm 0.076 & 0.614 & \pm 0.017 & \pm 0.054 \\
\hline $0.30-0.40$ & 0.342 & 1.563 & \pm 0.013 & \pm 0.028 & 0.841 & \pm 0.013 & \pm 0.043 & 0.305 & \pm 0.009 & \pm 0.031 \\
\hline $0.40-0.60$ & 0.476 & 0.4495 & \pm 0.0051 & \pm 0.0100 & $0.2936=$ & \pm 0.0053 & \pm 0.0146 & 0.0784 & \pm 0.0034 & \pm 0.0110 \\
\hline $0.60-0.80$ & 0.674 & 0.0767 & \pm 0.0021 & \pm 0.0021 & $0.0596=$ & \pm 0.0022 & \pm 0.0031 & 0.0054 & \pm 0.0011 & \pm 0.0022 \\
\hline
\end{tabular}

Table 28: Differential cross section $\frac{1}{\sigma_{\text {tot }}} \frac{d \sigma}{d x_{p}}$ as a function of $x_{p}=p_{\text {hadron }} / p_{\text {beam }}$ for $\pi^{ \pm}, \mathrm{K}^{ \pm}$and $(\mathrm{p}, \overline{\mathrm{p}})$. The first error shown is statistical; the second includes the systematic errors from the uncertainties in the mean value and resolution of the ionization measurement and from nuclear interactions. There is an additional $3 \%$ relative error from the uncertainty in the distribution of the number of $d E / d x$ samples $\left(5 \%\right.$ for $\mathrm{K}^{ \pm}$below $x_{p}=0.010$ and $(\mathrm{p}, \overline{\mathrm{p}})$ below $\left.x_{p}=0.018\right)$. 

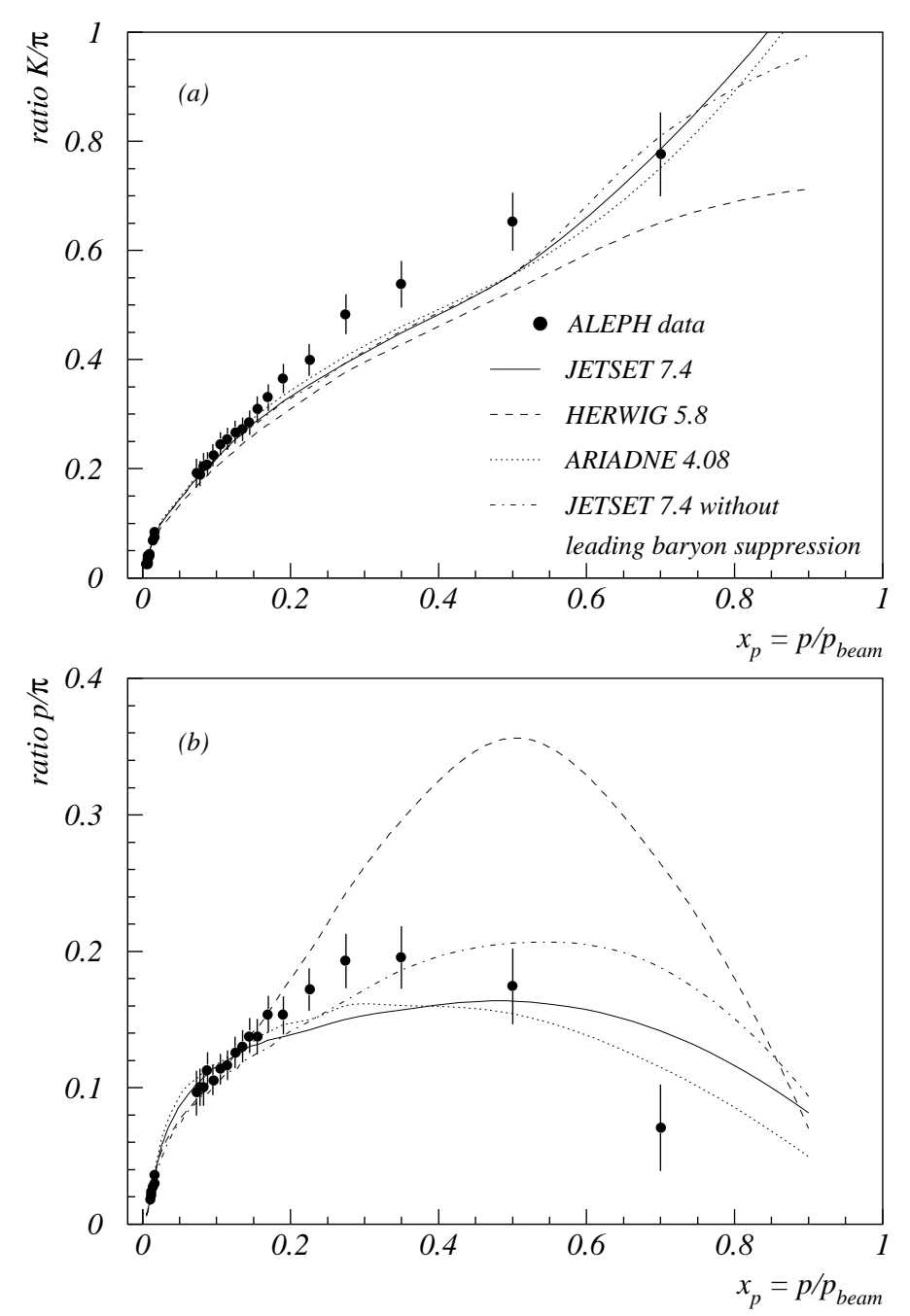

Figure 64: Ratios of the rates of (a) $\mathrm{K}^{ \pm} / \pi^{ \pm}$and (b) $(\mathrm{p}, \overline{\mathrm{p}}) / \pi^{ \pm}$as a function of $x_{p}=p_{\text {hadron }} / p_{\text {beam }}$, compared with the predictions of JETSET (with and without leading baryon suppression), HERWIG and ARIADNE. The errors shown are the quadratic sum of statistical and systematic errors.

about its peak [86] with a maximum $\xi^{\star}$ given by Eq. 64. According to the hypothesis of local parton-hadron duality [86], the inclusive distributions of final-state hadrons should have the same form, up to a normalization constant. Hence, $\xi^{\star}$ should vary as a function of $\ln \left(E_{c m}\right)$ according to Eq. (64), with a single free parameter $\Lambda$. The value of $\Lambda$ can be expected to change with particle type.

Peak positions $\xi^{\star}$ for the inclusive differential cross sections presented here were determined by fitting a Gaussian about the maximum in $d \sigma / d \xi$. The fitted $\xi^{\star}$ and their errors are shown in Table 29 together with ALEPH results for other hadron species which are discussed in the following sections.

Peak positions for pions, kaons and protons have been published by OPAL [162] at the Z peak and TOPAZ [163] at $58 \mathrm{GeV} / \mathrm{c}$. Differential cross sections published by TASSO [164, 165] (14$44 \mathrm{GeV} / \mathrm{c})$ and TPC [166, 167] $(29 \mathrm{GeV} / \mathrm{c})$ quote a combined statistical and systematic error. For these data a peak position was determined as described above, assuming the quoted errors to be uncorrelated. Variations in $\xi^{\star}$ resulting from changing the range fitted in $\xi$ are within the statistical error. Figure 65 shows the fitted $\xi^{\star}$ as a function of energy. The error shown is the quadratic sum of statistical and systematic errors.

Superimposed on Fig. 65 are fits according to Eq. (64). For both pions and protons there 


\begin{tabular}{|c|c|c|}
\hline & $\xi^{\star}$ & fitted $\xi$ range \\
\hline$\pi^{ \pm}$ & $3.776 \pm 0.004 \pm 0.024$ & $1.97-4.77$ \\
$\mathrm{~K}^{ \pm}$ & $2.70 \pm 0.01 \pm 0.09$ & $1.39-4.34$ \\
$\mathrm{~K}_{\mathrm{s}}^{0}$ & $2.67 \pm 0.01 \pm 0.05$ & $1.60-4.40$ \\
$(\mathrm{p}, \overline{\mathrm{p}})$ & $2.85 \pm 0.01 \pm 0.15$ & $1.39-3.73$ \\
$(\Lambda, \bar{\Lambda})$ & $2.72 \pm 0.02 \pm 0.05$ & $1.20-3.60$ \\
$\rho^{0}(770)$ & $2.80 \pm 0.19$ & $0.69-3.70$ \\
$K^{* 0}(892)$ & $2.26 \pm 0.05$ & $0.69-3.70$ \\
$K^{*+}(892)$ & $2.27 \pm 0.13$ & $1.00-3.40$ \\
$\phi(1020)$ & $2.21 \pm 0.03$ & $0.69-3.70$ \\
\hline
\end{tabular}

Table 29: Position of the peak $\xi^{\star}$ in $d \sigma / d \xi$ for identified hadrons. The first error quoted is statistical, the second systematic. For the vector mesons only the total error is given.

is good agreement with the MLLA calculation. The $\xi^{\star}$ values for kaons at the Z peak are low relative to this calculation and the lower energy data, and are excluded from the fit. Kaons arising from the decays of $b$ hadrons lie just to the left of the peak in $\xi$ with respect to kaons created from quarks in fragmentation, pulling $\xi^{\star}$ to lower values. It is estimated that the larger proportion of $b \bar{b}$ pairs produced at the $Z$ relative to $e^{+} e^{-}$annihilation at lower energies causes $\xi^{\star}$ to move downwards by $\sim 0.25$. This shift is shown on Fig. 65, and brings the data into reasonable agreement with an extrapolation of the fitted function. Also shown for pions on Fig. 65 , and clearly incompatible with the data, is the predicted dependence $\xi^{\star}=\ln \left(E_{c m} / 2 \Lambda\right)$ of an incoherent shower.

\subsubsection{Single Photons}

The primary sources of photons in hadronic events are neutral pions, which decay predominantly into $\gamma \gamma$. Decays of other hadrons, e.g., $\eta \rightarrow \gamma \gamma, \Sigma^{0} \rightarrow \Lambda \gamma$, as well as prompt photons radiated directly from quarks also contribute. By measuring the total photon rate one places a constraint on the sum of the production mechanisms, while avoiding the task of having to determine how the photons were created.

The inclusive photon spectrum has been measured with 534619 multihadronic events taken in 1992. The photons are detected by their conversion into an $e^{ \pm}$pair in the detector material. Up to the TPC volume the pair production probability is about $7 \%$.

The tracks of electron and positron candidates are required to have a transverse momentum with respect to the beam axis of $200 \mathrm{MeV} / \mathrm{c}$, at least $4 \mathrm{TPC}$ coordinates and a polar angle of at least $17^{\circ}$. In addition the ionization energy loss in the TPC and the shower shape in the electromagnetic calorimeter are used for the selection of $e^{ \pm}$tracks.

A pair-finding routine combines $e^{ \pm}$candidates and finds the point on each reconstructed helix where both tracks are parallel in the plane perpendicular to the beam axis ( $x y$-plane) and have the closest distance of approach. The centre between these two points is called the conversion point. Conversions in the TPC gas must fulfill the following requirements:

- The distance in the $x y$-plane between the two tracks at the closest approach to the conversion point must be less than $1.5 \mathrm{~cm}$. 


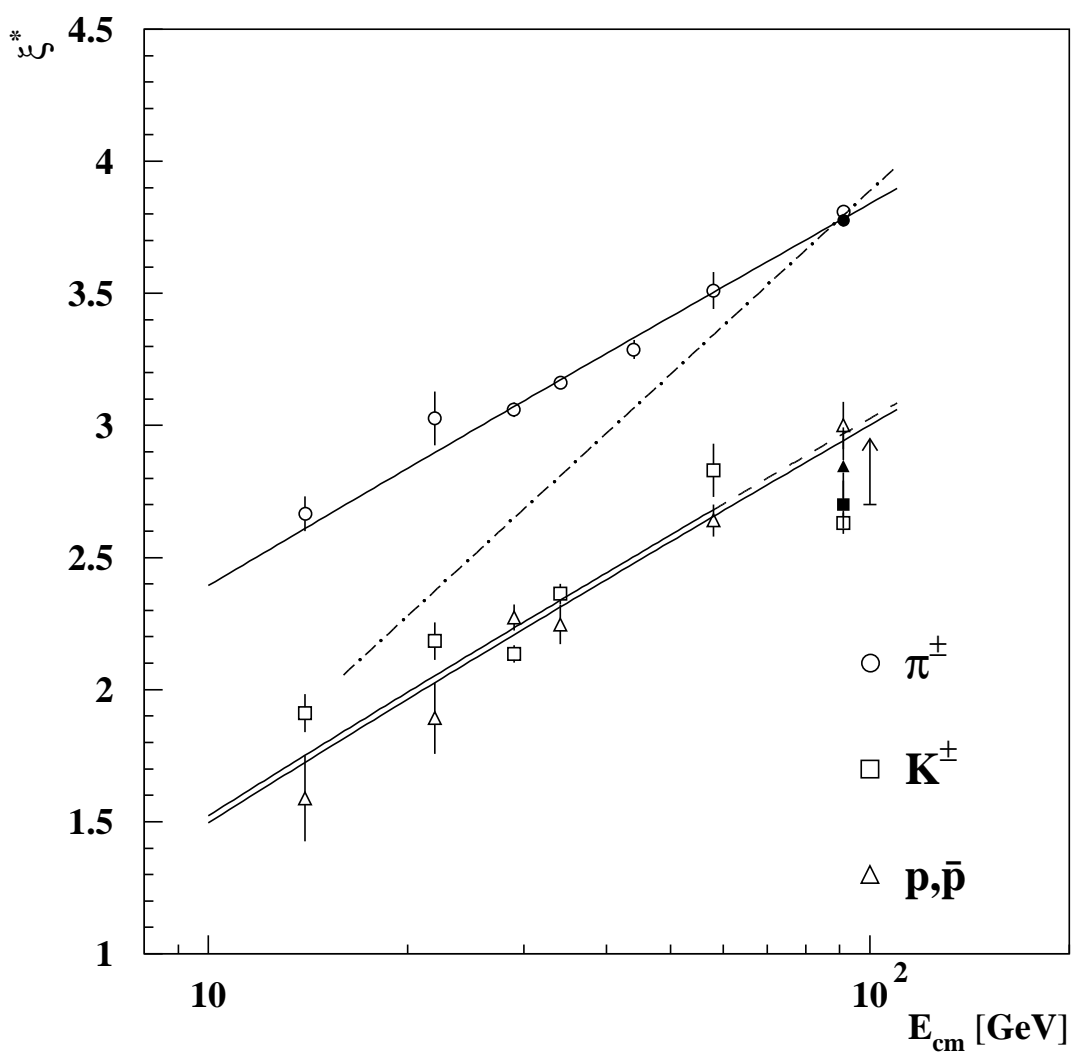

Figure 65: Position of the peak $\xi^{\star}$ in $\frac{d \sigma}{d \xi}$ as a function of centre-of-mass energy for pions, kaons and protons for the inclusive differential cross sections presented here (filled points) and those of other experiments (TASSO [164], [165], TPC/2 $\gamma$ [166], [167], TOPAZ [163] and OPAL [162]). The solid lines are fits to, from top to bottom, pion, kaon and proton data according to Eq. (64), assuming three flavours. The arrow represents the estimated shift in $\xi^{\star}$ for kaons at the $\mathrm{Z}$ peak due to $b$ hadron decays, and is to be compared with the extrapolation of Eq. (64) (dashed line). The dot-dashed line is the prediction for an incoherent parton shower model. 
- The $z$ separation of the tracks at the closest approach to the conversion point must be less than $0.9 \mathrm{~cm}$.

- The invariant mass of the two tracks at the conversion point assuming they are both electrons must be less than $11 \mathrm{MeV} / \mathrm{c}^{2}$.

For conversions in the other parts of the detector, the cuts are slightly different because of the different number of measured coordinates per track and the different amount of material that has been crossed. In this analysis up to $4 \%$ of the photons are reconstructed (depending on the photon energy) with a mean purity of about $92 \%$ and an average energy resolution, defined as the full width at half maximum divided by mean, of $1.5 \%$.

The normalization of the photon spectrum is obtained from the conversions in the TPC gas volume with radii between 40 and $100 \mathrm{~cm}$, where the amount of material is precisely known. The uncertainties from the normalization and the background estimation are a common contribution to the total systematic error in each bin of the measured spectrum.

The inclusive photon spectrum as a function of $\xi=-\ln \left(E_{\gamma} / E_{\text {beam }}\right)$ is given in Table 30 . The error is the combination of the statistical and systematic errors. Figure 66 compares the experimental result to different Monte Carlo predictions. The shape of the inclusive photon spectrum is well simulated by the different generators. However, the total number of photons per event in the generators is a bit low: in the energy range $0.84 \mathrm{GeV} \leq \mathrm{E}_{\gamma} \leq 20.5 \mathrm{GeV} \mathrm{ALEPH}$ finds $7.37 \pm 0.10$ (stat.) \pm 0.22 (syst.) photons, whereas the models predict 7.26 in JETSET 7.4, 7.22 in ARIADNE 4.08 and 7.18 in HERWIG 5.8.

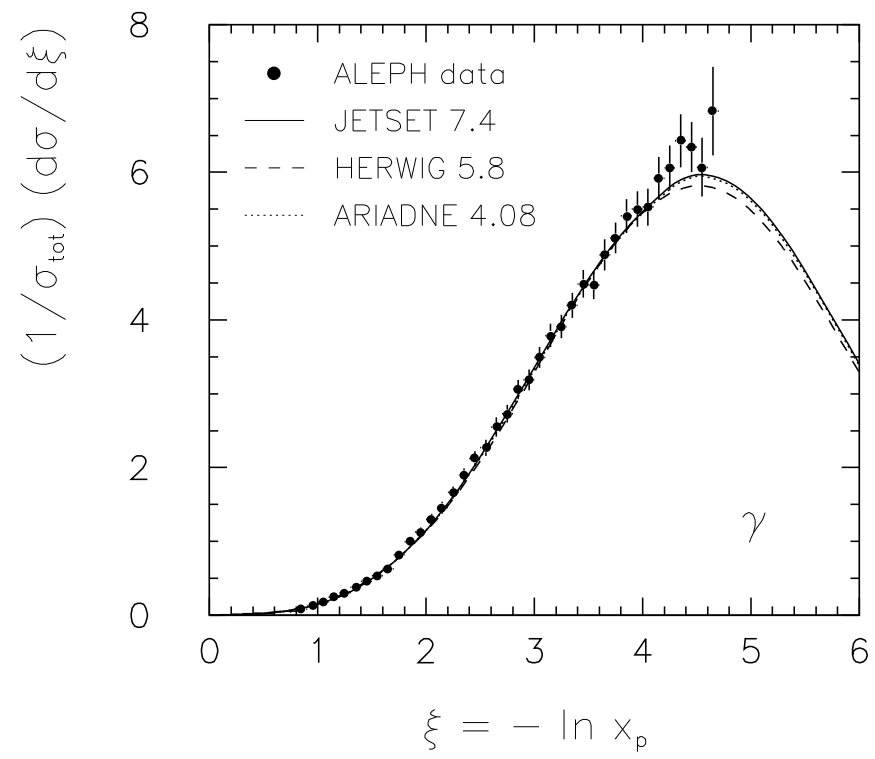

Figure 66: The inclusive photon spectrum. The error bars show the total errors including a common $3.5 \%$ uncertainty for the normalization and the background estimation. The full line shows the prediction of JETSET 7.4, the dashed line that of HERWIG 5.8 and the dotted line that of ARIADNE 4.08.

\subsubsection{Neutral Pions}

Neutral pion rates have been measured from their decay into two photons. (For details see [168]). In the ALEPH detector approximately $7 \%$ of all photons convert into an $\mathrm{e}^{+} \mathrm{e}^{-}$pair in the beam pipe or in the tracking chambers. Therefore two independent methods of photon identification are used: most photons are measured in the ECAL as an electromagnetic shower with no corresponding charged track, whereas the converted photons are reconstructed as $\mathrm{e}^{+} \mathrm{e}^{-}$ pairs as measured in the tracking chambers. The cross section for neutral pion production is 


\begin{tabular}{|c|c|}
\hline$\xi$ interval & $\left(1 / \sigma_{\text {tot }}\right)(d \sigma / d \xi)$ \\
\hline \hline $0.0-0.1$ & $0.0001 \pm 0.0018$ \\
$0.1-0.2$ & $0.0045 \pm 0.0039$ \\
$0.2-0.3$ & $0.0143 \pm 0.0096$ \\
$0.3-0.4$ & $0.0185 \pm 0.0078$ \\
$0.4-0.5$ & $0.0251 \pm 0.0075$ \\
$0.5-0.6$ & $0.0256 \pm 0.0071$ \\
$0.6-0.7$ & $0.046 \pm 0.014$ \\
$0.7-0.8$ & $0.055 \pm 0.017$ \\
$0.8-0.9$ & $0.087 \pm 0.016$ \\
$0.9-1.0$ & $0.136 \pm 0.020$ \\
$1.0-1.1$ & $0.182 \pm 0.036$ \\
$1.1-1.2$ & $0.246 \pm 0.031$ \\
$1.2-1.3$ & $0.300 \pm 0.039$ \\
$1.3-1.4$ & $0.383 \pm 0.027$ \\
$1.4-1.5$ & $0.457 \pm 0.055$ \\
$1.5-1.6$ & $0.538 \pm 0.048$ \\
$1.6-1.7$ & $0.625 \pm 0.043$ \\
$1.7-1.8$ & $0.815 \pm 0.055$ \\
$1.8-1.9$ & $0.996 \pm 0.059$ \\
$1.9-2.0$ & $1.126 \pm 0.066$ \\
$2.0-2.1$ & $1.300 \pm 0.075$ \\
$2.1-2.2$ & $1.458 \pm 0.081$ \\
$2.2-2.3$ & $1.662 \pm 0.083$ \\
$2.3-2.4$ & $1.899 \pm 0.092$ \\
\hline
\end{tabular}

\begin{tabular}{|c|c|}
\hline$\xi$ interval & $1 / \sigma_{\text {tot }} d \sigma / d \xi$ \\
\hline \hline $2.4-2.5$ & $2.12 \pm 0.10$ \\
$2.5-2.6$ & $2.27 \pm 0.11$ \\
$2.6-2.7$ & $2.55 \pm 0.13$ \\
$2.7-2.8$ & $2.73 \pm 0.12$ \\
$2.8-2.9$ & $3.06 \pm 0.13$ \\
$2.9-3.0$ & $3.19 \pm 0.14$ \\
$3.0-3.1$ & $3.49 \pm 0.14$ \\
$3.1-3.2$ & $3.79 \pm 0.16$ \\
$3.2-3.3$ & $3.91 \pm 0.16$ \\
$3.3-3.4$ & $4.20 \pm 0.17$ \\
$3.4-3.5$ & $4.49 \pm 0.19$ \\
$3.5-3.6$ & $4.47 \pm 0.19$ \\
$3.6-3.7$ & $4.88 \pm 0.21$ \\
$3.7-3.8$ & $5.11 \pm 0.21$ \\
$3.8-3.9$ & $5.41 \pm 0.23$ \\
$3.9-4.0$ & $5.50 \pm 0.24$ \\
$4.0-4.1$ & $5.53 \pm 0.25$ \\
$4.1-4.2$ & $5.92 \pm 0.29$ \\
$4.2-4.3$ & $6.06 \pm 0.30$ \\
$4.3-4.4$ & $6.43 \pm 0.36$ \\
$4.4-4.5$ & $6.34 \pm 0.34$ \\
$4.5-4.6$ & $6.07 \pm 0.40$ \\
$4.6-4.7$ & $6.83 \pm 0.60$ \\
$4.7-4.8$ & $8.3 \pm 3.5$ \\
\hline
\end{tabular}

Table 30: Measured inclusive photon cross section. The errors include statistical and systematic uncertainties.

extracted from the invariant mass distributions of photon pairs which is parameterized in terms of a Gaussian for the signal and a background function.

The total $\pi^{0}$ rate is determined from a sample with two conversions which has lower statistics, but gives the smallest systematic error (6.4\%), mainly because the detector material in the TPC gas volume is known to $1 \%$. The higher statistics of $\pi^{0}$ mesons reconstructed via one converted and one calorimetric photon is used to measure the shape of the $\pi^{0}$ spectrum. In this way the analysis is less sensitive to the uncertainties of the photon reconstruction in the ECAL. The corrected results are given in Table 31 and plotted in Fig. 67. The measured mean multiplicity per event for $\pi^{0} \mathrm{~s}$ with momentum fraction larger than 0.025 is found to be $4.80 \pm 0.07 \pm 0.31$, where the first error is statistical and the second is systematic.

The $\pi^{0}$ spectrum is extrapolated into the momentum range below $x_{p}=0.025$ using the shape predicted by the JETSET 7.4 model. The experimental result is $\left\langle n_{\pi^{0}}\right\rangle=9.63 \pm 0.13 \pm 0.62 \pm 0.12$, where the third error is the uncertainty of the extrapolation taken from the difference between JETSET 7.4 and HERWIG 5.8 and the variation of the model parameters. This ALEPH measurement is in good agreement with the predictions of JETSET $7.4\left(\left\langle n_{\pi^{0}}\right\rangle=9.67\right)$ and HERWIG $5.8\left(\left\langle n_{\pi^{0}}\right\rangle=9.59\right)$. The neutral pion data have not been used in the tuning of the model parameters (see sect. 2.3). 


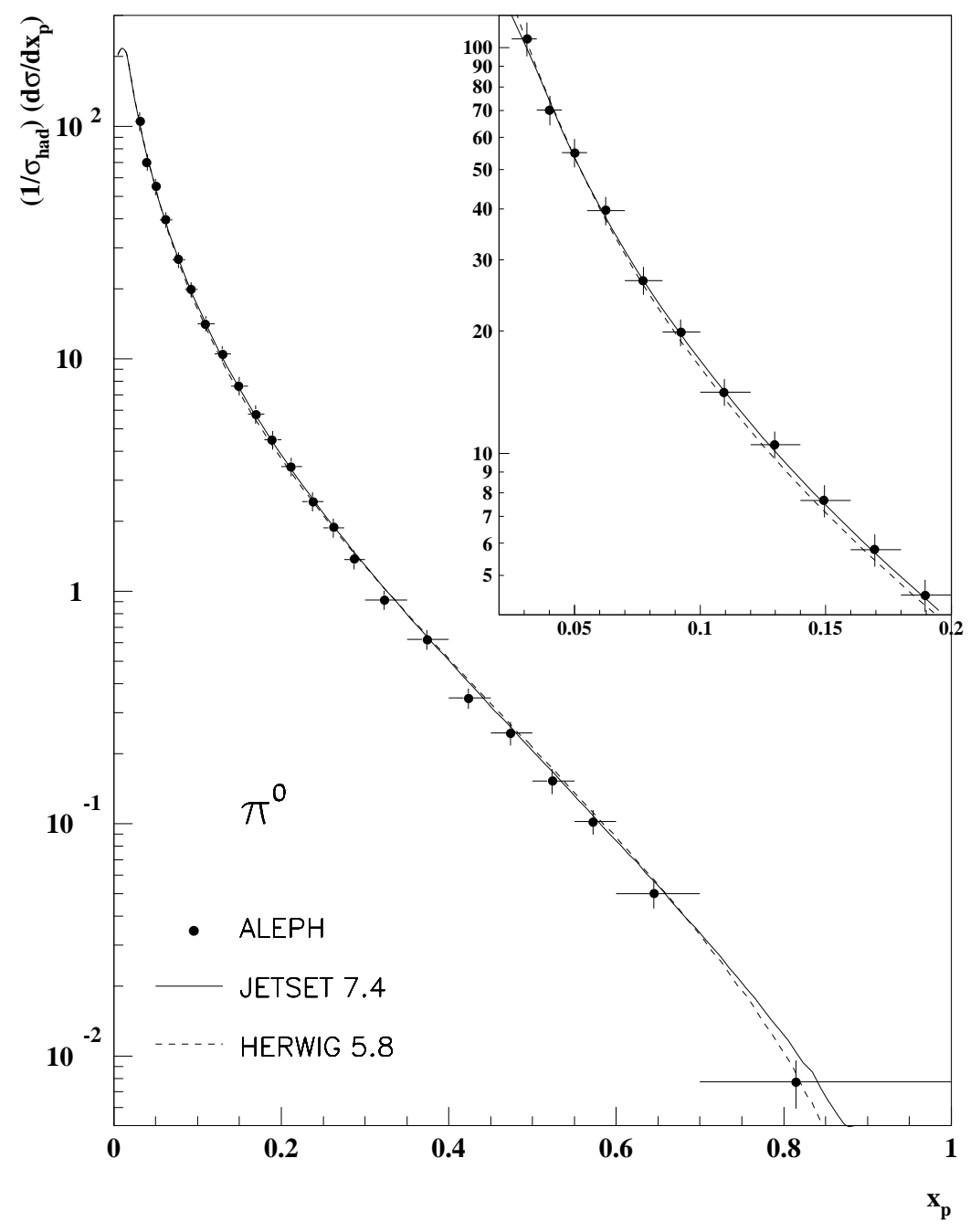

Figure 67: Differential inclusive

cross section of neutral pions as function of $x_{p}$.

Figure 68 shows the measured $\pi^{0}$ spectrum as a function of $\xi=\ln \left(1 / x_{p}\right)$ in comparison to the charged pion spectrum. At high momenta (low $\xi$ ) positive, neutral and negative pions are produced with the same frequency, as expected from isospin invariance of the strong interaction, whereas at lower momenta more neutral pions are found. This demonstrates the importance of electromagnetic and weak decays for the low momentum pions, e.g. in the decay $\eta \rightarrow 3 \pi^{0}$.

\subsection{4 $\eta$ and $\eta^{\prime}$ Mesons}

The decays $\eta \rightarrow \gamma \gamma$ and $\eta^{\prime} \rightarrow \eta \pi^{+} \pi^{-}$have been observed in a sample of 920,000 hadronic $\mathrm{Z}$ decays following an earlier ALEPH analysis [169].

The decay $\eta \rightarrow \gamma \gamma$ is identified by measuring the decay photons in the ALEPH electromagnetic calorimeter. Using photons with $E>2 \mathrm{GeV}$, the masses of all photon pairs in an event are determined and these are measured as a function of the corresponding fractional energy $x_{E}=E(\gamma \gamma) / E_{\text {beam }}$. Photons which in combination with another photon of more than $1 \mathrm{GeV}$ have a mass within $25 \mathrm{MeV}$ of the mass of the $\pi^{0}$ are removed. The $\eta$ resonance is clearly visible in the mass spectra and the rate in each $x_{E}$ interval is determined by a fit, with care taken to avoid reflections from the decay $\omega \rightarrow \pi^{0} \gamma$. By using simulated Monte Carlo events to correct the acceptance loss and the measured branching ratio for $\eta \rightarrow \gamma \gamma$, it is possible to 


\begin{tabular}{|l|r|r|}
\hline$x_{p}$ range & $\left\langle x_{p}\right\rangle$ & \multicolumn{1}{|c|}{$\frac{1}{\sigma_{\text {tot }}} \frac{d \sigma}{d x_{p}} \pm \Delta_{\text {stat }} \pm \Delta_{\text {syst }}$} \\
\hline \hline $0.025-0.035$ & 0.031 & $105.132 \pm 5.912 \pm 8.095$ \\
$0.035-0.045$ & 0.040 & $70.133 \pm 2.102 \pm 5.400$ \\
$0.045-0.055$ & 0.050 & $55.040 \pm 1.329 \pm 4.238$ \\
$0.055-0.070$ & 0.062 & $39.626 \pm 0.723 \pm 3.051$ \\
$0.070-0.085$ & 0.077 & $26.674 \pm 0.470 \pm 2.054$ \\
$0.085-0.100$ & 0.092 & $19.884 \pm 0.356 \pm 1.471$ \\
$0.100-0.120$ & 0.110 & $14.153 \pm 0.225 \pm 1.047$ \\
$0.120-0.140$ & 0.130 & $10.505 \pm 0.181 \pm 0.777$ \\
$0.140-0.160$ & 0.150 & $7.644 \pm 0.143 \pm 0.680$ \\
$0.160-0.180$ & 0.170 & $5.788 \pm 0.118 \pm 0.515$ \\
$0.180-0.200$ & 0.190 & $4.474 \pm 0.100 \pm 0.398$ \\
$0.200-0.225$ & 0.212 & $3.439 \pm 0.077 \pm 0.361$ \\
$0.225-0.250$ & 0.237 & $2.435 \pm 0.061 \pm 0.256$ \\
$0.250-0.275$ & 0.262 & $1.877 \pm 0.054 \pm 0.197$ \\
$0.275-0.300$ & 0.287 & $1.370 \pm 0.043 \pm 0.144$ \\
$0.300-0.350$ & 0.323 & $0.917 \pm 0.025 \pm 0.080$ \\
$0.350-0.400$ & 0.373 & $0.620 \pm 0.021 \pm 0.052$ \\
$0.400-0.450$ & 0.423 & $0.347 \pm 0.016 \pm 0.028$ \\
$0.450-0.500$ & 0.474 & $0.245 \pm 0.019 \pm 0.019$ \\
$0.500-0.550$ & 0.523 & $0.153 \pm 0.013 \pm 0.011$ \\
$0.550-0.600$ & 0.573 & $0.102 \pm 0.009 \pm 0.007$ \\
$0.600-0.700$ & 0.643 & $0.050 \pm 0.005 \pm 0.003$ \\
$0.700-1.000$ & 0.770 & $0.008 \pm 0.002 \pm 0.001$ \\
\hline
\end{tabular}

Table 31: Measured differential cross section for $\pi^{0}$ production. 




Figure 68: Differential inclusive cross section of neutral and charged pions as function of $\xi=\ln \left(1 / x_{p}\right)$. The error bars show the total errors (statistical and systematic errors added in quadrature).

determine the differential cross section:

$$
\frac{1}{\sigma_{\text {tot }}} \frac{d \sigma}{d x_{E}}(\mathrm{Z} \rightarrow \eta+X)
$$

The results are shown in Table 32 and Fig. 69, where the spectrum is compared with the predictions from JETSET 7.4, HERWIG 5.8 and ARIADNE 4.08. Another measurement of the $\eta$ rate has been obtained in [170] using the $\pi^{+} \pi^{-} \pi^{0}$ decay channel, with compatible results although with a larger error.

To look for $\eta^{\prime}$ candidates, photon pairs which are within $100 \mathrm{MeV}$ of the $\eta$ mass are taken as $\eta$ candidates and their masses are constrained. These candidates are combined with all oppositely-signed charged-track pairs in the same event. These tracks are predominantly pions and are well measured in the ALEPH TPC. The masses of the candidate $\eta \pi^{+} \pi^{-}$combinations as a function of $x_{E}=E\left(\eta \pi^{+} \pi^{-}\right) / E_{\text {beam }}$ show a clear signal for the $\eta^{\prime}$, and as for the $\eta$, the corresponding differential cross section is determined and is shown in Table 32 and Fig. 69.

The measured $\eta$ and $\eta^{\prime}$ multiplicities per hadronic $Z$ decay for $x_{E}>0.1$ are $0.282 \pm 0.015 \pm$ 0.016 and $0.064 \pm 0.013 \pm 0.005$ respectively, in agreement with the earlier results [169] of $0.298 \pm 0.023 \pm 0.021$ and $0.068 \pm 0.018 \pm 0.016$. Whereas the original JETSET Monte Carlo predicts far too many $\eta^{\prime}$ mesons[169], the JETSET 7.4 version with the extra $\eta^{\prime}$ suppression factor of 0.27 describes both the $\eta$ and $\eta^{\prime}$ rates $\left(0.298\right.$ and 0.071 respectively for $x_{E}>0.1$ ).

\subsubsection{Light Strange Particles}

$\mathrm{K}_{s}^{0}$ mesons and $\Lambda$ hyperons are cleanly identified in the ALEPH apparatus through their decay into two charged particles: $\mathrm{K}_{s}^{0} \rightarrow \pi^{+} \pi^{-}$and $\Lambda \rightarrow p \pi^{-}$. The TPC provides up to 338 measurements of the specific ionization $(d E / d x)$ of each charged track. For charged tracks 


\begin{tabular}{|l|lll|}
\hline \multicolumn{4}{|c|}{$\eta$} \\
\hline \hline$x_{E}$ range & \multicolumn{2}{|c|}{$\left(1 / \sigma_{\text {tot }}\right)\left(d \sigma / d x_{E}\right)$} \\
\hline $0.10-0.14$ & 1.71 & \pm & 0.28 \\
$0.14-0.18$ & 1.65 & \pm & 0.22 \\
$0.18-0.22$ & 0.963 & \pm & 0.046 \\
$0.22-0.26$ & 0.721 & \pm & 0.051 \\
$0.26-0.30$ & 0.560 & \pm & 0.028 \\
$0.30-0.34$ & 0.387 & \pm & 0.019 \\
$0.34-0.38$ & 0.254 & \pm & 0.018 \\
$0.38-0.42$ & 0.193 & \pm & 0.015 \\
$0.42-0.46$ & 0.171 & \pm & 0.019 \\
$0.46-0.50$ & 0.128 & \pm & 0.013 \\
$0.50-0.54$ & 0.113 & \pm & 0.012 \\
$0.54-0.58$ & 0.0616 & \pm & 0.0062 \\
$0.58-0.62$ & 0.0388 & \pm & 0.0043 \\
$0.62-0.66$ & 0.0316 & \pm & 0.0042 \\
$0.66-0.70$ & 0.0281 & \pm & 0.0036 \\
$0.70-0.74$ & 0.0194 & \pm & 0.0036 \\
$0.74-0.82$ & 0.0079 & \pm & 0.0011 \\
$0.82-1.00$ & 0.0021 & \pm & 0.0005 \\
\hline Systematic error $\pm 6 \%$ \\
\hline
\end{tabular}

\begin{tabular}{|r|lll|}
\hline \multicolumn{3}{|c|}{$\eta^{\prime}$} \\
\hline \hline$x_{E}$ range & \multicolumn{2}{|c|}{$\left(1 / \sigma_{\text {tot }}\right)\left(d \sigma / d x_{E}\right)$} \\
\hline $0.10-0.20$ & 0.32 & \pm & 0.12 \\
$0.20-0.30$ & 0.146 & \pm & 0.028 \\
$0.30-0.40$ & 0.082 & \pm & 0.013 \\
$0.40-0.50$ & 0.034 & \pm & 0.007 \\
$0.50-0.60$ & 0.030 & \pm & 0.005 \\
$0.60-0.70$ & 0.0144 & \pm & 0.0026 \\
$0.70-0.80$ & 0.0094 & \pm & 0.0019 \\
$0.80-0.90$ & 0.0034 & \pm & 0.0014 \\
$0.90-1.00$ & 0.0002 & \pm & 0.0005 \\
\hline \multicolumn{5}{|c|}{ Systematic error $\pm 8 \%$} \\
\hline
\end{tabular}

Table 32: Measured fragmentation functions for the $\eta$ and $\eta^{\prime}$ mesons.


Figure 69: The $\eta$ (a) and $\eta^{\prime}$ (b) fragmentation function compared with the predictions of JETSET 7.4, HERWIG 5.8 and ARIADNE 4.08. All errors shown are statistical only.

with momenta above $3 \mathrm{GeV} / \mathrm{c}$ and with the maximum number of samples, the truncated mean ionizations of pions and protons are separated by three standard deviations. In the analysis very loose cuts are applied in order to distinguish between $\mathrm{K}_{s}^{0}$ mesons and $\Lambda$ hyperons. The specific ionization on each track of a $V^{0}$ candidate is required to be within three standard deviations of the expected ionization, if at least 50 ionization samples are measured by the TPC, and the $\chi^{2}$ of the kinematical $V^{0}$ fit, constrained by the mass hypothesis, is required to 
be less than 120 for 3 degrees of freedom. Details of the analysis, which was based on 988000 hadronic Z decays collected during 1991 and 1992, have been published in ref. [171].

The differential cross sections as a function of $x_{p}=p / p_{\text {beam }}$, where $p$ is the particle momentum, are given in Table 33. Figure 70 shows the momentum spectra as a function of $\xi=-\ln \left(p / p_{\text {beam }}\right)$ together with predictions of JETSET, HERWIG and ARIADNE. The JETSET and ARIADNE predictions agree nicely with the measured spectrum of $K^{0}$, whereas the HERWIG spectrum is slightly lower. The JETSET and ARIADNE Monte Carlos also give a good description of the measured $\Lambda$ spectrum, whereas HERWIG overestimates the $\Lambda$ production. The agreement of JETSET and ARIADNE with the data is largely due to the tuning of the model parameters. In particular, the leading baryon suppression mechanism has to be activated (see sect. 2.3). It is obvious that HERWIG has a leading particle effect for high momentum $\Lambda$ 's which is not present in the data. The same effect was already seen in the proton spectrum. The HERWIG model also has a large yield of $\Lambda$ at low momenta. This is caused by the very large rate of $\Xi$ states and of spin $3 / 2$ baryons in HERWIG. The measured $\Lambda$ spectrum does not support such a large production of heavy hyperons, and neither do the direct measurements of $\Xi$ and $\Sigma$ production, as described below and in [172].

The measured $\xi$ distributions are integrated from $\xi=0$ to $\xi=5.4$ (4.4 for $\Lambda$ ). The JETSET spectrum is used to extrapolate the average multiplicities from the momentum cutoff to zero momentum, after normalizing it to data in the interval $5<\xi<5.4(4<\xi<4.4$ for $\Lambda)$. The results are shown in Table 41 in Section 5.2.9, where the summary of the measured multiplicities is compared with the predictions of JETSET, ARIADNE and HERWIG. The agreement is good in general, except that HERWIG overestimates the $\Lambda$ rate.


Figure 70: Momentum spectra of $K^{0}$ (left) and $\Lambda$ (right). The systematic errors are added in quadrature. Note that the systematic errors are correlated.

\subsubsection{Heavy Strange Particles}

Inclusive production of $\Sigma^{0}, \Xi^{-}, \Xi(1530)^{0}, \Sigma(1385)^{ \pm}$and $\Omega^{-}$hyperons has been studied with the ALEPH detector using the decay modes $\Sigma^{0} \rightarrow \Lambda \gamma, \Xi^{-} \rightarrow \Lambda \pi^{-}, \Xi^{* 0} \rightarrow \Xi^{-} \pi^{+}, \Sigma^{* \pm} \rightarrow \Lambda \pi^{ \pm}$ and $\Omega^{-} \rightarrow \Lambda K^{-}$. These measurements provide information on the formation of baryons with one, two, or three strange quarks and with spin $1 / 2$ or spin $3 / 2$.

For the analysis of $\Xi^{-}, \Xi(1530)^{0}, \Sigma(1385)^{ \pm}$and $\Omega^{-}$hyperons, a total of 2.86 million candidates for hadronic $\mathrm{Z}$ decays is selected by requiring at least five good charged tracks 


\begin{tabular}{|c|c|c|c|c|}
\hline \multicolumn{7}{|c|}{$\left(1 / \sigma_{\text {tot }}\right)\left(d \sigma / d x_{p}\right)$} \\
\hline$x_{p}$ range & $\left\langle x_{p}\right\rangle$ & $K^{0}$ & $\Lambda$ \\
\hline \hline $0.004-0.005$ & 0.0045 & $8.71 \pm 2.41$ & & \\
$0.005-0.006$ & 0.0055 & $11.01 \pm 1.41$ & & \\
$0.006-0.008$ & 0.0071 & $14.92 \pm 1.27$ & & \\
$0.008-0.010$ & 0.0090 & $17.64 \pm 1.13$ & & \\
$0.010-0.012$ & 0.0110 & $20.68 \pm 1.11$ & & \\
$0.012-0.014$ & 0.0130 & $22.94 \pm 1.10$ & $2.97 \pm 0.35$ \\
$0.014-0.016$ & 0.0150 & $23.96 \pm 1.10$ & $3.43 \pm 0.30$ \\
$0.016-0.018$ & 0.0170 & $24.44 \pm 1.06$ & $3.74 \pm 0.29$ \\
$0.018-0.020$ & 0.0190 & $23.85 \pm 0.96$ & $3.70 \pm 0.21$ \\
$0.020-0.025$ & 0.0225 & $22.25 \pm 0.86$ & $3.69 \pm 0.18$ \\
$0.025-0.030$ & 0.0275 & $20.07 \pm 0.72$ & $3.68 \pm 0.16$ \\
$0.030-0.035$ & 0.0325 & $18.60 \pm 0.65$ & $3.70 \pm 0.15$ \\
$0.035-0.040$ & 0.0375 & $16.94 \pm 0.59$ & $3.41 \pm 0.14$ \\
$0.040-0.050$ & 0.0449 & $14.69 \pm 0.45$ & $3.18 \pm 0.11$ \\
$0.050-0.060$ & 0.0549 & $12.44 \pm 0.35$ & $2.66 \pm 0.09$ \\
$0.060-0.080$ & 0.0695 & $10.08 \pm 0.27$ & $2.04 \pm 0.06$ \\
$0.080-0.100$ & 0.0896 & $7.60 \pm 0.19$ & $1.52 \pm 0.04$ \\
$0.100-0.120$ & 0.1096 & $5.92 \pm 0.13$ & $1.19 \pm 0.03$ \\
$0.120-0.140$ & 0.1297 & $4.89 \pm 0.10$ & $0.956 \pm 0.023$ \\
$0.140-0.160$ & 0.1497 & $3.969 \pm 0.080$ & $0.771 \pm 0.018$ \\
$0.160-0.180$ & 0.1697 & $3.346 \pm 0.065$ & $0.630 \pm 0.015$ \\
$0.180-0.200$ & 0.1897 & $2.780 \pm 0.055$ & $0.528 \pm 0.013$ \\
$0.200-0.250$ & 0.2232 & $2.001 \pm 0.040$ & $0.408 \pm 0.010$ \\
$0.250-0.300$ & 0.2732 & $1.313 \pm 0.030$ & $0.269 \pm 0.008$ \\
$0.300-0.350$ & 0.3234 & $0.875 \pm 0.023$ & $0.182 \pm 0.007$ \\
$0.350-0.400$ & 0.3734 & $0.592 \pm 0.019$ & $0.129 \pm 0.006$ \\
$0.400-0.500$ & 0.4439 & $0.337 \pm 0.014$ & $0.078 \pm 0.005$ \\
$0.500-0.600$ & 0.5439 & $0.166 \pm 0.011$ & $0.035 \pm 0.003$ \\
$0.600-0.700$ & 0.6436 & $0.080 \pm 0.009$ & $0.0118 \pm 0.0019$ \\
$0.700-0.900$ & 0.7686 & $0.026 \pm 0.008$ & $0.0026 \pm 0.0012$ \\
\hline
\end{tabular}

Table 33: The inclusive spectrum of $K_{S}^{0}+K_{L}^{0}$ and of $\Lambda+\bar{\Lambda}$ from hadronic Z decay as a function of $x_{p}=p / p_{\text {beam }}$. The errors include momentum dependent systematic errors. In addition there is an overall normalization error of $2 \%$ for neutral kaons and of $4 \%$ for $\Lambda$ hyperons. 
with a total of at least $10 \%$ of the centre-of-mass energy. The $\Lambda$ candidates are identified by the decay $\Lambda \rightarrow p \pi^{-}$. The selection is identical to that described in [171], except that no cut is made on the $\Lambda$ impact parameter, and no kinematic fitting is done. Only those candidates with a reconstructed mass lying within two standard deviations of the $\Lambda$ mass are used in the following analysis. For the $\Xi$ and $\Omega$ analyses the resulting neutral $\Lambda$ track is vertexed with all remaining good tracks of the appropriate charge, and candidates are selected by requiring that the decay length measured from the beam spot be within the range of 0.2 to 5 proper lifetimes. In addition, the $\Lambda$ decay length as measured between the two vertices is required to be in the range of 0.2 to 5 proper lifetimes, and each vertex fit is required to have a $\chi^{2}$ per degree of freedom of no greater than 10 , with the reconstructed $\Xi$ or $\Omega$ extrapolating to within $0.8 \mathrm{~cm}$ of the beam. Kaon candidates are rejected if there are less than 50 ionization measurements or if the measured $d E / d x$ is not within $2 \sigma$ of the expected value. Finally, for the $\Omega^{-}$selection, candidates are rejected if they fall within $7.5 \mathrm{MeV} / c^{2}$ of the $\Xi^{-}$mass, assuming the third track has the mass of pion.

The resulting $\Xi^{-}$signal is shown in Fig. 71(a). Depending on the $\Xi$ momentum, the mass resolution varies between $2 \mathrm{MeV} / c^{2}$ and $6 \mathrm{MeV} / c^{2}$. The mass histogram for each $x_{E}=E / E_{b e a m}$ bin is fit to a gaussian plus quadratic polynomial. Thus the sidebands, which agree well with the wrong-sign spectrum, are used to determine the background. Figure 71(b) shows the $\Lambda K^{-}$ mass spectrum, where a clear peak is seen at the $\Omega^{-}$mass, with a resolution consistent with that expected from the Monte Carlo simulation. The fit to a gaussian plus quadratic polynomial is used to estimate the background in the signal region, which is taken to be $\pm 6 \mathrm{MeV} / \mathrm{c}^{2}$ about the peak. The number of events above background in this region is $156 \pm 17$.

The $\Sigma(1385)^{ \pm}$is reconstructed by pairing the $\Lambda$ candidates with tracks of $p>200 \mathrm{MeV} / \mathrm{c}$ that pass within $0.2 \mathrm{~cm}$ of the beam and which are no more than $60^{\circ}$ in angle from the $\Lambda$ momentum vector. The data are shown in Fig. 71(c). The combinatorial background is fit to a function of the form

$$
N \cdot\left(x-x_{0}\right)^{P} \cdot \exp \left(c_{1}\left(x-x_{0}\right)+c_{2}\left(x-x_{0}\right)^{2}\right)
$$

while the signal is taken to be a relativistic Breit-Wigner shape with a mass-dependent width [173]:

$$
\Gamma(m)=\Gamma_{0} \cdot\left(\frac{q}{q_{0}}\right) \cdot\left(\frac{m_{0}}{m}\right)
$$

where $q\left(q_{0}\right)$ is the momentum of the $\Lambda$ in the $\Sigma^{*}$ rest frame for invariant mass $m\left(m_{0}\right)$ and $m_{0}, \Gamma_{0}$ are fixed to the known values. The decay sequence $\Sigma^{*} \rightarrow \Sigma^{0} \pi^{ \pm}$, with $\Sigma^{0} \rightarrow \Lambda \gamma$, is accounted for by an additional signal of the same shape shifted downward and broadened to account for the missing photon, by amounts determined from Monte Carlo simulation. Finally, a gaussian peak at the $\Xi^{-}$mass is included. It is important to note here that the number quoted for the $\Sigma(1385)$ production rate includes only those particles falling between the mass threshold at $1.254 \mathrm{GeV}$ and $1.685 \mathrm{GeV}\left(9 \Gamma_{0}\right.$ above $\left.m_{0}\right)$.

For the $\Xi(1530)^{0}$ analysis, all $\Xi^{-}$candidates within $6.4 \mathrm{MeV} / c^{2}$ of the $\Xi^{-}$mass are paired with all remaining good tracks of $p>300 \mathrm{MeV} / \mathrm{c}$ and of the appropriate charge that extrapolate to within $0.8 \mathrm{~cm}$ of the beam. The cosine of the angle between the pion candidate and the $\Xi(1530)$ momentum, measured in the $\Xi(1530)$ rest frame, must be less than 0.85 . The measured $\Xi^{-} \pi^{-}$spectrum is used to fix the shape, but not the normalization, of the background, by fitting it to the same function as used for the $\Sigma^{*}$ background. The $\Xi^{-} \pi^{+}$signal in the $M_{\Lambda \pi \pi}-M_{\Lambda \pi}$ spectrum then is fit to a convolution of a Breit-Wigner, with fixed width, and a gaussian plus background shape, as shown in Fig. 71(d) for the full $x_{E}$ range. 

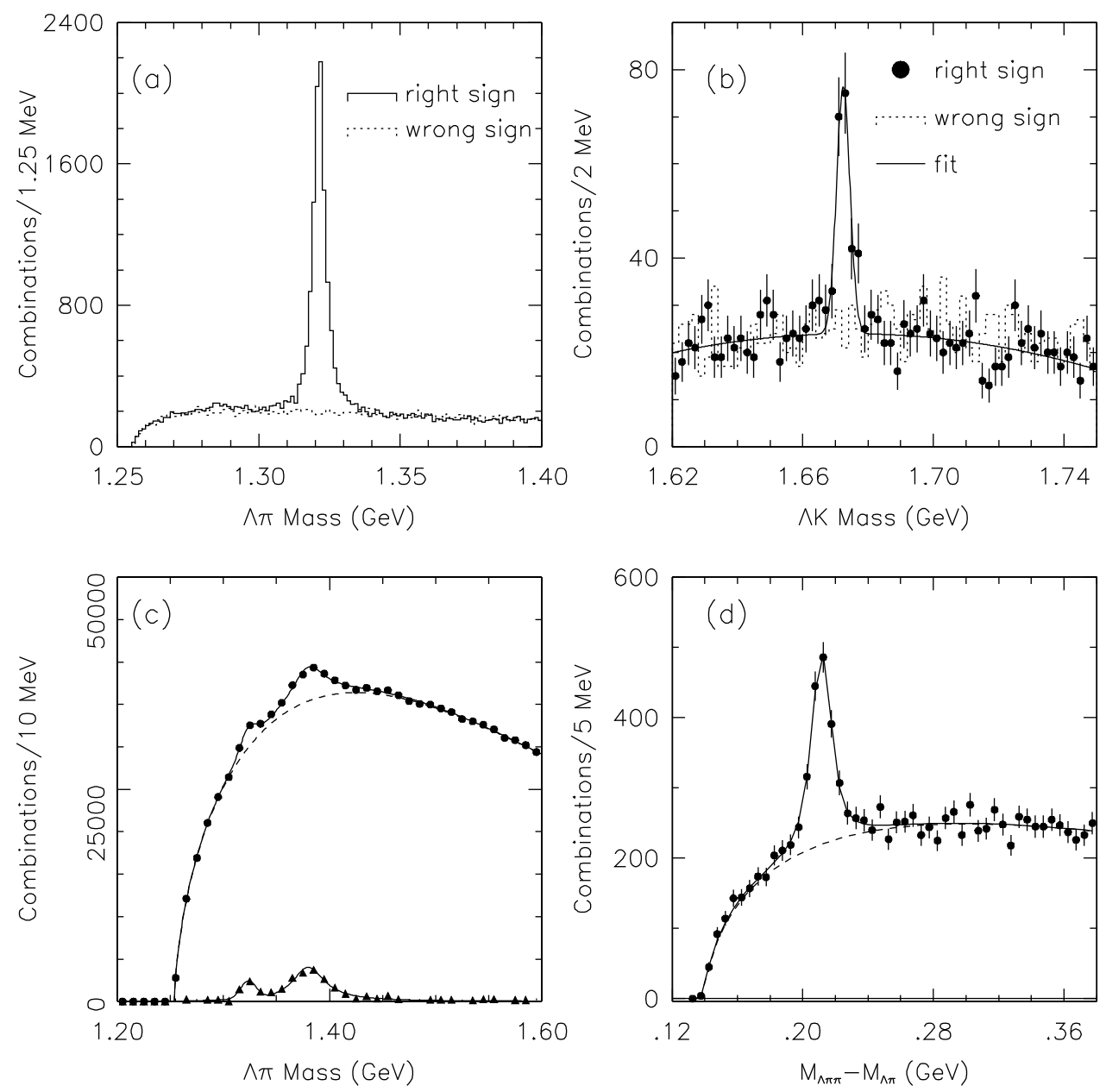

Figure 71: (a) The signal for $\Xi^{-} \rightarrow \Lambda \pi^{-}$. (b) The signal for $\Omega^{-} \rightarrow \Lambda K^{-}$. (c) The mass spectrum for $\Sigma(1385)^{ \pm} \rightarrow \Lambda \pi^{ \pm}$candidates, fitted to the background shape, a $\Xi^{-}$contribution, plus a Breit-Wigner resonance. Also shown is the background-subtracted spectrum. (d) The signal for $\Xi(1530)^{0} \rightarrow \Xi^{-} \pi^{+}$, fitted to the background shape plus a Breit-Wigner resonance convoluted with a gaussian.

For each of the analyses, the efficiencies are calculated by Monte Carlo simulation, in which the simulated events are treated the same as data, the methods of background subtraction included. The corrected measurements of the $x_{E}$ distributions for $\Sigma^{*}, \Xi^{-}$, and $\Xi(1530)^{0}$ are given in Tables 34 through 36 . Figure 72 compares the experimental results to the predictions of the JETSET 7.4 model, the HERWIG 5.8 model and the ARIADNE model. Whereas JETSET and ARIADNE agree with the data, the HERWIG predictions are significantly higher. This leading particle effect in HERWIG which is not present in the data has already been seen for the protons and $\Lambda$ hyperons.

The JETSET model is used to extrapolate the results over the unmeasured regions at low $x_{E}$ to obtain the overall multiplicities:

$$
\begin{aligned}
\left\langle N_{\Xi^{-}}\right\rangle+\left\langle N_{\Xi^{+}}\right\rangle & =0.0297 \pm 0.00057 \pm 0.0020, \\
\left\langle N_{\Xi(1530)^{0}}\right\rangle+\left\langle N_{\Xi(1530)^{0}}\right\rangle & =0.0072 \pm 0.0004 \pm 0.0006, \\
\left\langle N_{\Sigma(1385)^{ \pm}}\right\rangle+\left\langle N_{\bar{\Sigma}(1385)^{ \pm}}\right\rangle & =0.065 \pm 0.004 \pm 0.008 \\
\left\langle N_{\Omega^{-}}\right\rangle+\left\langle N_{\bar{\Omega}^{+}}\right\rangle & =0.0010 \pm 0.0002 \pm 0.0001,
\end{aligned}
$$


where in each case the first uncertainty is statistical and the second is systematic. The systematic errors include a common $4 \%$ uncertainty in the efficiency of $\Lambda$ reconstruction. Table 41 shows that these results are in reasonable agreement with the model predictions ( except for HERWIG ).

As a cross check, the $c \tau$ of the $\Lambda$ and $\Xi^{-}$are measured to be in good agreement with the accepted values. The $\Xi^{-}$polarization is measured to be $0.04 \pm 0.06$, consistent with zero. An uncertainty of \pm 0.06 in the polarization introduces a negligible systematic error in the efficiency.
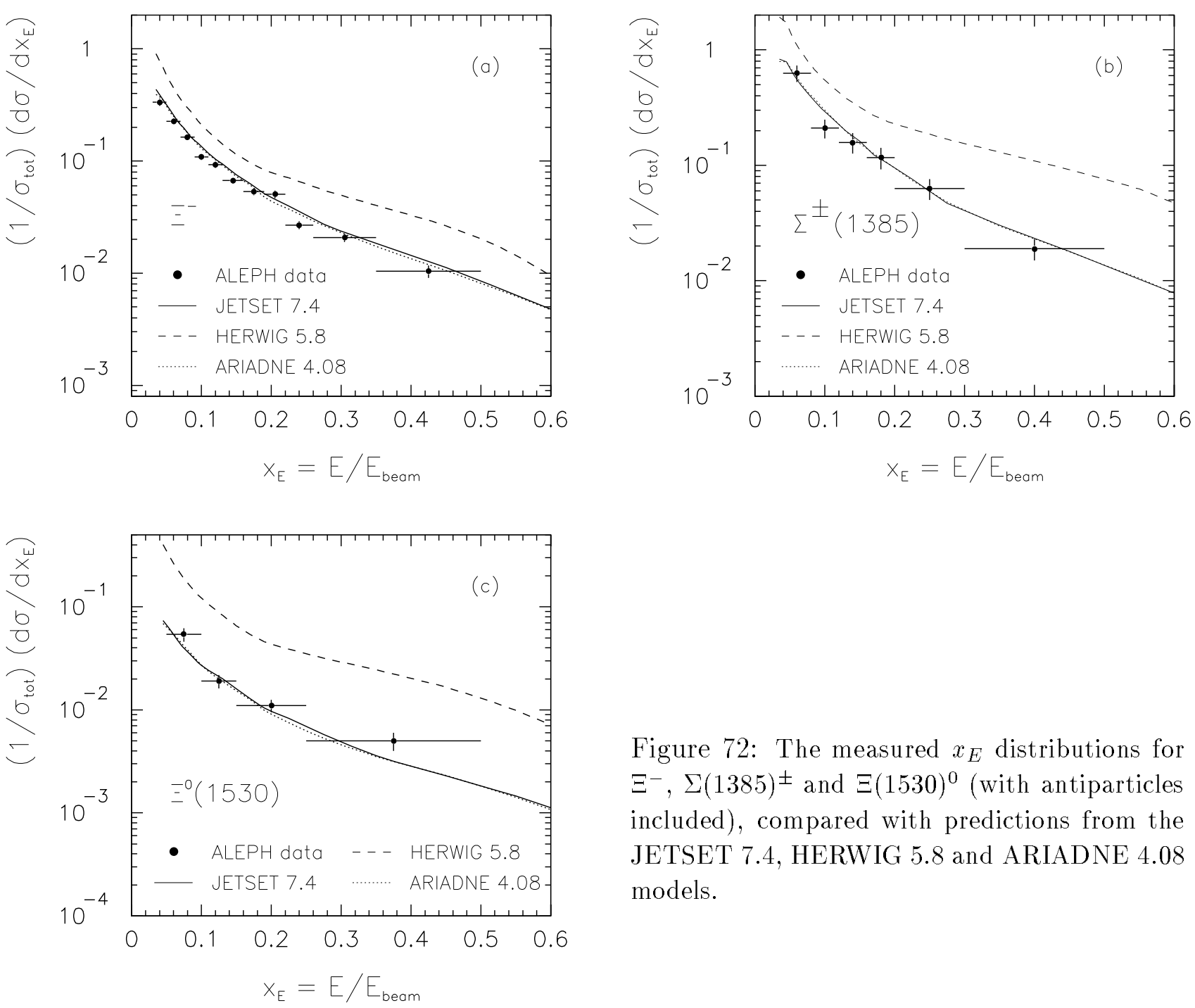

Figure 72: The measured $x_{E}$ distributions for $\Xi^{-}, \Sigma(1385)^{ \pm}$and $\Xi(1530)^{0}$ (with antiparticles included), compared with predictions from the JETSET 7.4, HERWIG 5.8 and ARIADNE 4.08 models.

The $\Sigma^{0} \rightarrow \Lambda \gamma$ analysis is based on 2337867 hadronic events from the 1992-1994 running period. To ensure a good energy resolution for the low energy photons (typically $150 \mathrm{MeV}$ ), only those photons which convert into $e^{+} e^{-}$pairs in the material of the ALEPH detector are used. Combinations of photons and $\Lambda$ hyperons are accepted as $\Sigma^{0}$ candidates, if the $\Sigma^{0}$ momentum is greater than $3.0 \mathrm{GeV} / \mathrm{c}$ and if the decay angle $\vartheta^{*}$ between the flight direction of $\Sigma^{0}$ and $\Lambda$ in the $\Sigma^{0}$ rest frame fulfills the condition $-0.95 \leq \cos \left(\vartheta^{*}\right) \leq 0.5$.

The resulting mass difference $\left(m_{\Lambda \gamma}-m_{\Lambda}\right)$ ( Fig. 73$)$ shows a $\Sigma^{0}$ peak at $(76.4 \pm 0.5)$ $\mathrm{MeV}$ with $158 \pm 19$ decays. Using the JETSET model to correct for the detection efficiency $\epsilon=(8.22 \pm 0.82) \cdot 10^{-4}$, the following average multiplicity per Z decay is found:

$$
\left\langle N_{\Sigma^{0}}\right\rangle+\left\langle N_{\bar{\Sigma}^{0}}\right\rangle=0.082 \pm 0.010 \pm 0.012
$$




\begin{tabular}{|c|c|c|}
\hline$x_{E}$ Interval & $\left\langle x_{E}\right\rangle$ & $\left(1 / \sigma_{\text {tot }}\right)\left(d \sigma / d x_{E}\right)$ \\
\hline $0.03-0.05$ & 0.0394 & $0.336 \pm 0.026$ \\
\hline $0.05-0.07$ & 0.0594 & $0.226 \pm 0.014$ \\
\hline $0.07-0.09$ & 0.0795 & $0.163 \pm 0.0099$ \\
\hline $0.09-0.11$ & 0.0996 & $0.109 \pm 0.0068$ \\
\hline $0.11-0.13$ & 0.120 & $0.0925 \pm 0.0060$ \\
\hline $0.13-0.16$ & 0.144 & $0.0672 \pm 0.0043$ \\
\hline $0.16-0.19$ & 0.174 & $0.0533 \pm 0.0038$ \\
\hline $0.19-0.22$ & 0.204 & $0.0507 \pm 0.0040$ \\
\hline $0.22-0.26$ & 0.239 & $0.0269 \pm 0.0023$ \\
\hline $0.26-0.35$ & 0.301 & $0.0208 \pm 0.0018$ \\
\hline $0.35-0.50$ & 0.417 & $0.0104 \pm 0.0013$ \\
\hline
\end{tabular}

Table 34: The measured $x_{E}$ distribution for the $\Xi^{-}$.

\begin{tabular}{|c|c|c|}
\hline$x_{E}$ Interval & $\left\langle x_{E}\right\rangle$ & $\left(1 / \sigma_{\text {tot }}\right)\left(d \sigma / d x_{E}\right)$ \\
\hline $0.04-0.08$ & .0578 & $0.629 \pm 0.102$ \\
\hline $0.08-0.12$ & .0983 & $0.210 \pm 0.039$ \\
\hline $0.12-0.16$ & .1380 & $0.158 \pm 0.032$ \\
\hline $0.16-0.20$ & .1790 & $0.117 \pm 0.025$ \\
\hline $0.20-0.30$ & .2450 & $0.063 \pm 0.013$ \\
\hline $0.30-0.50$ & .3783 & $0.019 \pm 0.004$ \\
\hline
\end{tabular}

Table 35: The measured $x_{E}$ distribution for the $\Sigma(1385)$.

\begin{tabular}{|c|c|c|}
\hline$x_{E}$ Interval & $\left\langle x_{E}\right\rangle$ & $\left(1 / \sigma_{\text {tot }}\right)\left(d \sigma / d x_{E}\right)$ \\
\hline $0.05-0.1$ & .072 & $0.054 \pm 0.0082$ \\
\hline $0.1-0.15$ & .123 & $0.019 \pm 0.0028$ \\
\hline $0.15-0.25$ & .192 & $0.011 \pm 0.0015$ \\
\hline $0.25-0.5$ & .351 & $0.005 \pm 0.001$ \\
\hline
\end{tabular}

Table 36: The measured $x_{E}$ distribution for the $\Xi(1530)$. 
where the systematic error of $15 \%$ is dominated by the Monte Carlo uncertainties of $10 \%$. The reconstruction uncertainties for $\Lambda$ 's and photons are $5 \%$ each.

The measured $\Sigma^{0}$ rate is well reproduced by the Monte Carlo models. JETSET predicts $0.087 \Sigma^{0}$ hyperons and HERWIG predicts $0.064 \Sigma^{0}$ hyperons per event.

Most $\Sigma^{0}$ s should originate from the fragmentation in the same way as for the $\Xi^{-}$. Thus the ratio of $\Sigma^{0}$ to $\Xi^{-}$rates provides a measure of the strangeness suppression $(s / u)$ in baryon production. The ALEPH results are $\Xi^{-} / \Sigma^{0}=0.36 \pm 0.10$. In the same way the ratio of $\Sigma^{*}$ to $\Sigma^{0}$ measures the spin suppression in the hyperon sector. From the ALEPH numbers the $\left(\mathrm{S}_{(3 / 2)} / \mathrm{S}_{(1 / 2)}\right)$ ratio is found to be $0.5 \cdot \Sigma^{* \pm} / \Sigma^{0}=0.40 \pm 0.10$.



Figure 73: The signal for $\Sigma^{0} \rightarrow \Lambda \gamma$, fitted to the background plus a Breit-Wigner function for the signal.

\subsubsection{Neutral Vector Mesons}

The cross sections for the inclusive production of the neutral mesons $\rho^{0}(770), \omega(782), \mathrm{K}^{* 0}(892)$, $\phi(1020)$ in hadronic $\mathrm{Z}$ decays are extracted from the invariant mass distributions of their daughters. The decay modes $\rho^{0} \rightarrow \pi^{+} \pi^{-}, \omega \rightarrow \pi^{+} \pi^{-} \pi^{0}, \mathrm{~K}^{* 0} \rightarrow \mathrm{K}^{ \pm} \pi^{\mp}$ and $\phi \rightarrow \mathrm{K}^{+} \mathrm{K}^{-}$ are measured. Charged pions and kaons are identified by their ionization energy loss in the TPC, and neutral pions are reconstructed from pairs of neutral clusters in the ECAL. The invariant mass distributions in intervals of $x_{p}=p_{\text {hadron }} / p_{\text {beam }}$ are fitted as the sum of a signal and a background function. The signal is taken as a convolution of a p-wave Breit-Wigner function and a resolution function which accounts for the experimental mass resolution. The $\rho^{0}$ line shape is affected by Bose-Einstein correlations. The $\omega$ reconstruction is slightly different because of the narrow width: no $\mathrm{dE} / \mathrm{dx}$ information is used in the selection of charged pions and the $\omega$ signal is taken as the sum of three gaussian functions. The corrections for reconstruction efficiency are determined from JETSET 7.3 tuned to ALEPH data [26] including full detector simulation. Details of the analysis can be found in ref. [170]. 
The acceptance-corrected cross sections are given in Tables 37 and 38 , in which the first error quoted is statistical and the second systematic. In Fig. 74 the momentum spectra are shown and compared to model predictions from JETSET 7.4, HERWIG 5.8 and ARIADNE 4.08. The error bars in Fig. 74 show the quadratic sum of statistical and systematic uncertainties.
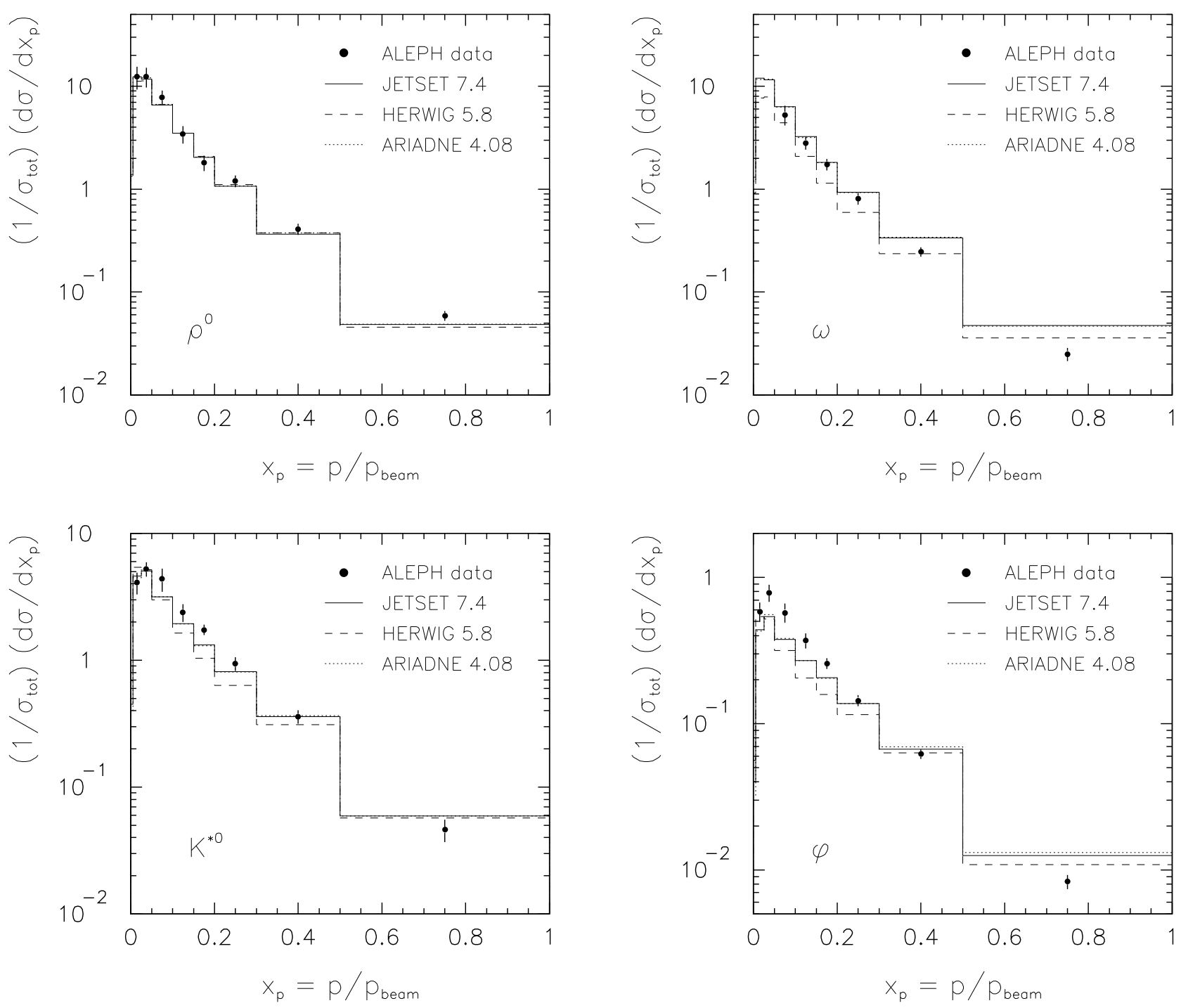

Figure 74: Differential cross section for $\rho^{0}, \omega, \mathrm{K}^{* 0}$, and $\phi$ in comparison with Monte Carlo predictions. The errors shown are the quadratic sum of statistical and systematic uncertainties.

The meson production rates are extracted by adding the rates from all measured $x_{p}$ bins and extrapolating to $x_{p}=0$. The range $0.005 \leq x_{p} \leq 1$ comprises more than $99 \%$ of the total rate for $\rho^{0}, \mathrm{~K}^{* 0}$, and $\phi$. For the $\omega$, where the measurement starts at $x_{p}=0.05$, JETSET 7.4 is used for extrapolation. The total multiplicities are collected in Table 41 and compared to predictions from the models JETSET 7.4, ARIADNE 4.08 and HERWIG 5.8 .

The $\rho^{0}$ meson is found to have an average multiplicity per event of $1.453 \pm 0.065$ (stat) \pm 0.201(syst). The Monte Carlo predictions of all three models considered are very similar and in good agreement with the measured momentum spectrum and the total rate. The average multiplicity per event for the $\omega$ has been measured for $x_{p}>0.05$ to be $0.637 \pm 0.034$ (stat) \pm 0.074 (syst). An extrapolation of this multiplicity to $x_{p}=0$ yields $1.066 \pm 0.058$ (stat) \pm 0.124 (syst) \pm 0.044 (extrap.) per event. The measured momentum spectrum and the total rate 


\begin{tabular}{|l|c|c|}
\hline \multicolumn{3}{|c|}{$\left(1 / \sigma_{\text {tot }}\right)\left(d \sigma / d x_{p}\right)$} \\
\hline$x_{p}$ range & $\rho^{0}(770)$ & $\omega(782)$ \\
\hline \hline $0.005-0.025$ & $12.42 \pm 2.04 \pm 2.33$ & \\
\hline $0.025-0.05$ & $12.41 \pm 1.33 \pm 2.36$ & \\
\hline $0.05-0.10$ & $7.82 \pm 0.58 \pm 1.11$ & $5.312 \pm 0.627 \pm 0.977$ \\
\hline $0.10-0.15$ & $3.44 \pm 0.36 \pm 0.55$ & $2.817 \pm 0.223 \pm 0.332$ \\
\hline $0.15-0.20$ & $1.81 \pm 0.20 \pm 0.24$ & $1.743 \pm 0.118 \pm 0.183$ \\
\hline $0.20-0.30$ & $1.20 \pm 0.10 \pm 0.12$ & $0.812 \pm 0.044 \pm 0.098$ \\
\hline $0.30-0.50$ & $0.41 \pm 0.03 \pm 0.04$ & $0.247 \pm 0.012 \pm 0.023$ \\
\hline $0.50-1.00$ & $0.059 \pm 0.004 \pm 0.005$ & $0.025 \pm 0.002 \pm 0.003$ \\
\hline
\end{tabular}

Table 37: Measured differential cross sections for $\rho^{0}$ and $\omega$.

\begin{tabular}{|l|l|l|}
\hline \multicolumn{3}{|c|}{$\left(1 / \sigma_{\text {tot }}\right)\left(d \sigma / d x_{p}\right)$} \\
\hline$x_{p}$ range & \multicolumn{1}{|c|}{$\mathrm{K}^{* 0}(892)$} & $\phi(1020)$ \\
\hline \hline $0.005-0.025$ & $4.10 \pm 0.36 \pm 0.71$ & $0.584 \pm 0.055 \pm 0.069$ \\
\hline $0.025-0.05$ & $5.24 \pm 0.29 \pm 0.61$ & $0.787 \pm 0.089 \pm 0.056$ \\
\hline $0.05-0.10$ & $4.36 \pm 0.15 \pm 0.89$ & $0.576 \pm 0.046 \pm 0.073$ \\
\hline $0.10-0.15$ & $2.39 \pm 0.13 \pm 0.36$ & $0.370 \pm 0.026 \pm 0.035$ \\
\hline $0.15-0.20$ & $1.74 \pm 0.07 \pm 0.15$ & $0.258 \pm 0.019 \pm 0.011$ \\
\hline $0.20-0.30$ & $0.94 \pm 0.04 \pm 0.11$ & $0.144 \pm 0.0089 \pm 0.0085$ \\
\hline $0.30-0.50$ & $0.36 \pm 0.01 \pm 0.043$ & $0.0620 \pm 0.0033 \pm 0.0030$ \\
\hline $0.50-1.00$ & $0.046 \pm 0.002 \pm 0.009$ & $0.00831 \pm 0.00057 \pm 0.00074$ \\
\hline
\end{tabular}

Table 38: Measured differential cross sections for $\mathrm{K}^{* 0}$ and $\phi$.

lies between the prediction of JETSET and ARIADNE (1.29 $\omega$ per event) and HERWIG (0.86 $\omega$ per event).

The rate of $\omega$ production is expected to be almost the same as for the $\rho^{0}$ since the two have essentially the same flavour content, the same spin, and nearly the same mass, only differing in isospin. The ratio of the measured production rate of the $\rho^{0}$ to that of the $\omega$ is $1.36 \pm 0.27$. This agrees within errors with the value of 1.06 from JETSET, which does not distinguish isospin states.

The average multiplicity per event of the $\mathrm{K}^{* 0}$ is found to be $0.830 \pm 0.015$ (stat) \pm 0.088 (syst). This rate is slightly higher than the predictions of JETSET and ARIADNE $\left(0.72 \mathrm{~K}^{* 0}\right.$ per event) and of HERWIG (0.68 $\mathrm{K}^{* 0}$ per event). The measured momentum spectrum, however, is not well described.

The average $\phi$ multiplicity per event has been measured to be $0.122 \pm 0.004$ (stat) \pm 0.008 (syst). This result is somewhat larger than the predictions of JETSET and ARIADNE (0.098 $\phi$ per event) and HERWIG (0.088 $\phi$ per event), however the spectrum is not reliably reproduced by any of the models.

For the determination of the strangeness suppression, the assumption is made that the 
relative production of non-strange and strange vector mesons is governed by the frequency by which an up or down quark is replaced by a strange quark. The up and down quark being equally produced, the ratios $N\left(\mathrm{~K}^{* 0}\right) / 2 \mathrm{~N}\left(\rho^{0}\right), N\left(\mathrm{~K}^{* 0}\right) / 2 \mathrm{~N}(\omega)$, and $2 N(\phi) / N\left(\mathrm{~K}^{* 0}\right)$ therefore should represent $N(\mathrm{~s}) / \mathrm{N}(\mathrm{u})$; the latter ratio is usually abbreviated $\mathrm{s} / \mathrm{u}$. The ratios are expected to agree only when corrected for decays and leading quarks. No corrections are made for decays from higher spin states such as tensor mesons.

The results for the measured vector meson rates are: $N\left(\mathrm{~K}^{* 0}\right) / 2 \mathrm{~N}\left(\rho^{0}\right)=0.29 \pm 0.01 \pm 0.05$, $N\left(\mathrm{~K}^{* 0}\right) / 2 \mathrm{~N}(\omega)=0.39 \pm 0.02 \pm 0.06$, and $2 N(\phi) / N\left(\mathrm{~K}^{* 0}\right)=0.29 \pm 0.01 \pm 0.04$. This compares well with the values $0.26,0.28$ and 0.27 for the tuned JETSET 7.4 , respectively, where the parameter for the strangeness suppression was 0.285 .

A comparison of the inclusive spectra of vector and pseudoscalar mesons provides information about the relative probabilities for the corresponding spin states to be produced in the hadronization. In JETSET the probability to produce a strange meson with spin 1 is controlled by the parameter $[\mathrm{V} /(\mathrm{V}+\mathrm{P})]_{\mathrm{s}}$. This ratio pertains to mesons directly produced in the hadronization, and leads to a predicted ratio of vector to vector plus pseudoscalar kaons of $N\left(\mathrm{~K}^{* 0}\right) /\left(\mathrm{N}\left(\mathrm{K}^{* 0}\right)+\mathrm{N}\left(\mathrm{K}^{0}\right)\right)=0.26$. Using the number of $\mathrm{K}^{0}$ 's in Table 41, a measured value of $N\left(\mathrm{~K}^{* 0}\right) /\left(\mathrm{N}\left(\mathrm{K}^{* 0}\right)+\mathrm{N}\left(\mathrm{K}^{0}\right)\right)=0.29 \pm 0.02$ is obtained.

The modified leading logarithm approximation combined with the local parton-hadron duality model [107] predicts that the position of the maximum of the $\xi_{p}=\ln \left(1 / \mathrm{x}_{\mathrm{p}}\right)$ distribution should depend on the particle mass. expected to be predominantly produced by the fragmentation process. The position of the maximum is obtained by fitting a gaussian in the range $0.69<\xi_{p}<3.7$. The maxima follow the expected behaviour, i.e., the momentum spectrum is harder for particles with higher masses. The maxima are at $\xi_{p}$ max $=2.80 \pm 0.19$ for $\rho^{0}(770), 2.26 \pm 0.05$ for $\mathrm{K}^{* 0}(892)$, and $2.21 \pm 0.03$ for $\phi(1020)$. The maximum for the $\omega(782)$ lies too close to the edge of the fit range $\left(0.69<\xi_{p}<3.0\right)$ for a reliable value to be given.

In the same analysis the differential cross sections for $K^{\star 0}$ and $\phi(1020)$ as a function of the transverse momentum with respect to the thrust axis were measured. The results (see Table 39 ) are plotted in Fig. 75. The predictions of both JETSET and ARIADNE agree reasonably well with the measurement.
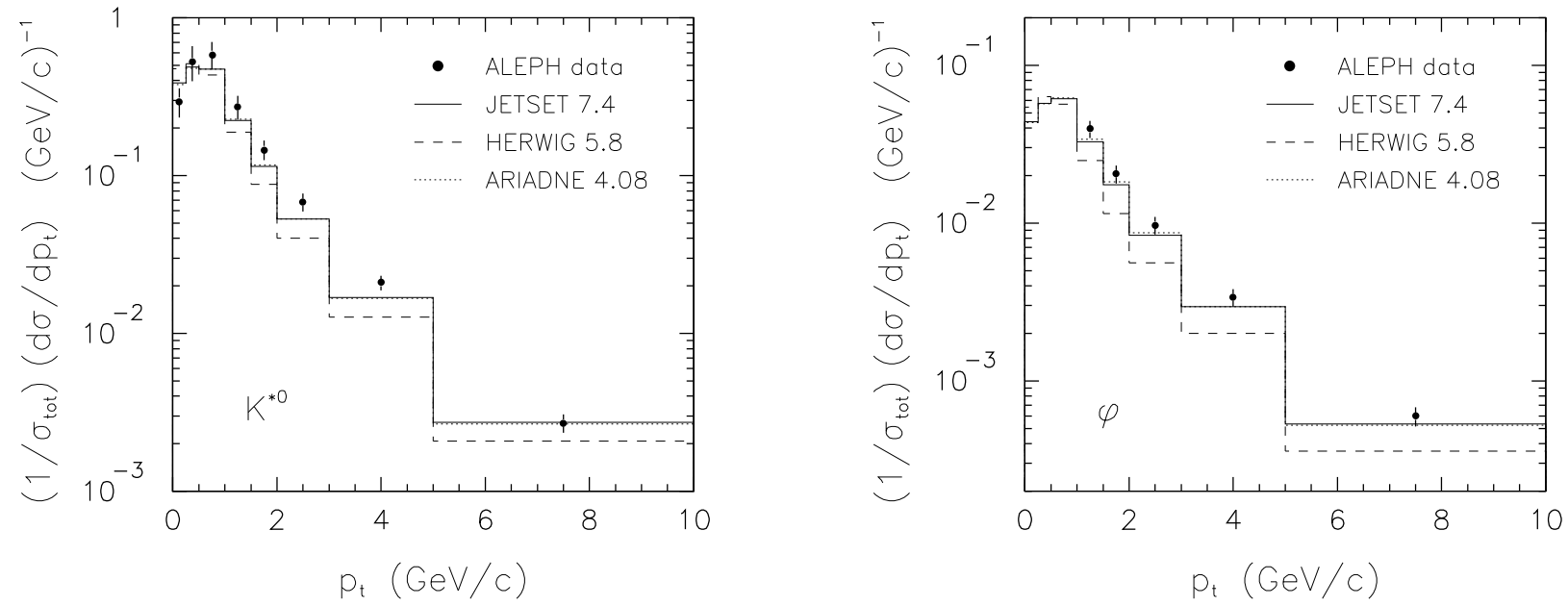

Figure 75: The inclusive spectrum as a function of $p_{\perp}$ for $\mathrm{K}^{* 0}$ and $\phi$. The spectra are compared to the predictions of JETSET 7.4, HERWIG 5.8 and ARIADNE 4.08. 


\begin{tabular}{|c|c|c|}
\hline \multirow{2}{*}{$\begin{array}{c}p_{\perp} \text { range } \\
(G \in V / c)\end{array}$} & \multicolumn{2}{|c|}{$\left(1 / \sigma_{t o t}\right)\left(d \sigma / d p_{\perp}\right)(G e V / c)^{-1}$} \\
\hline & $K^{\star 0}$ & $\phi(1020)$ \\
\hline $0 .-0.25$ & $0.296 \pm 0.027 \pm 0.056$ & \\
\hline $0.25-0.5$ & $0.528 \pm 0.036 \pm 0.128$ & \\
\hline $0.5-1.0$ & $0.586 \pm 0.018 \pm 0.115$ & \\
\hline $1.0-1.5$ & $0.273 \pm 0.009 \pm 0.046$ & $0.0395 \pm 0.0028 \pm 0.0040$ \\
\hline $1.5-2.0$ & $0.146 \pm 0.006 \pm 0.020$ & $0.0205 \pm 0.0017 \pm 0.0021$ \\
\hline $2.0-3.0$ & $0.068 \pm 0.004 \pm 0.008$ & $0.00970 \pm 0.00073 \pm 0.00097$ \\
\hline $3.0-5.0$ & $0.021 \pm 0.001 \pm 0.002$ & $0.00338 \pm 0.00026 \pm 0.00034$ \\
\hline $5.0-10.0$ & $0.0027 \pm 0.0002 \pm 0.0003$ & $0.000597 \pm 0.000057 \pm 0.000060$ \\
\hline
\end{tabular}

Table 39: Measured differential cross sections for $K^{\star 0}$ and $\phi(1020)$ as a function of the transverse momentum with respect to the thrust axis.

\subsubsection{Charged Vector Mesons}

$K^{* \pm}(892)$ mesons are measured in the decay chain: $K^{*+} \rightarrow K^{0} \pi^{+} ; K^{0} \rightarrow \pi^{+} \pi^{-} . K^{0}$ candidates are reconstructed using the $V^{0}$ algorithm described in Section 1.2. Additional cuts are applied to increase the purity of the $K^{0}$ sample:

- The $\chi^{2} /$ d.o.f. of the kinematical fit has to be less than 5 .

- The $K^{0}$ decay length has to be greater than $3 \mathrm{~cm}$.

- The $\pi^{+} \pi^{-}$mass is required to be within $\pm 40 \mathrm{MeV}$ of the $K^{0}$ mass.

- The cosine of the decay angle has to be less than 0.9 .

After these cuts, the average acceptance for $K_{S}^{0} \rightarrow \pi^{+} \pi^{-}$is $34.8 \%$ and the purity of the sample is $94.5 \%$.

Charged tracks which are not identified as electrons or muons are taken as pions and combined with the $K^{0}$ candidates. The $\left(K^{0} \pi^{ \pm}\right)$invariant mass distribution (Fig. 76) shows a clear resonance structure at the $K^{* \pm}(892)$ mass on top of a smooth combinatorial background. Whereas the analysis of the neutral vector mesons requires a detailed study of kinematical reflections and Bose-Einstein correlations, the $K^{* \pm}$ signal is obtained from a fit of a single Breit-Wigner function plus a background function of the form

$$
f(m)=p_{1} \cdot\left(m-m_{\text {thresh }}\right)^{p_{2}} \cdot \exp \left(-p_{3} m-p_{4} m^{2}\right)
$$

to the $\left(K^{0} \pi^{ \pm}\right)$mass distribution. Here $m_{\text {thresh }}$ is the threshold mass and $p_{i}$ are fit parameters.

In the ALEPH analysis, based on 290220 hadronic $Z$ decays, the $K^{* \pm}$ cross section is measured in 10 bins of $x_{E}$. The acceptance for each $x_{E}$ interval is obtained using Monte Carlo events generated with JETSET 7.3. The systematic error is dominated by the uncertainty in the experimental width of the Breit-Wigner function. Other sources like the $V^{0}$ finding efficiency or the parametrization of the background function have only small effects.

The results are given in Table 40 and plotted in Fig. 77. The measured spectrum is in good agreement with the Monte Carlo predictions. Integrating over the measured $x_{E}$ range gives the 


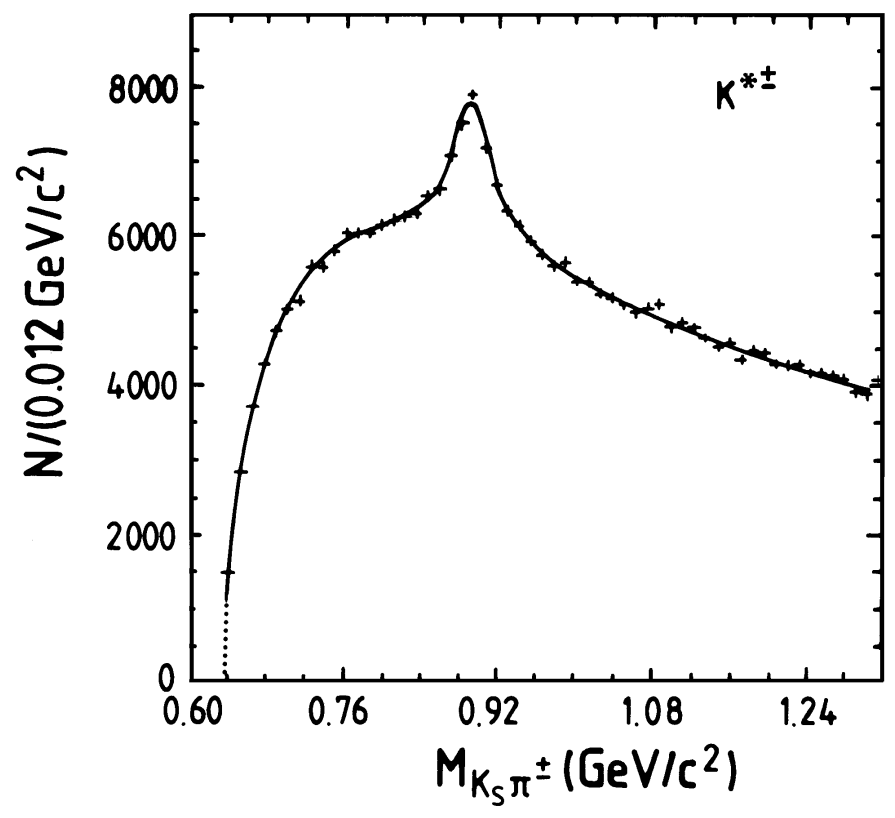

Figure 76: Distribution of the $\left(K^{0} \pi^{ \pm}\right)$-mass in the $x_{E}$ range $x_{E}=0.03-1.00$. The curve shows the result of the fit described in the text.

\begin{tabular}{|c|c|c|}
\hline$x_{E}$ range & $\left\langle x_{E}\right\rangle$ & $\frac{1}{\sigma_{\text {tot }}} \frac{d \sigma}{d x_{E}}$ \\
\hline \hline $0.03-0.06$ & 0.043 & $5.17 \pm 0.53 \pm 0.54$ \\
$0.06-0.09$ & 0.074 & $3.43 \pm 0.29 \pm 0.58$ \\
$0.09-0.12$ & 0.104 & $2.09 \pm 0.20 \pm 0.22$ \\
$0.12-0.15$ & 0.134 & $2.01 \pm 0.16 \pm 0.23$ \\
$0.15-0.18$ & 0.164 & $1.54 \pm 0.15 \pm 0.19$ \\
$0.18-0.22$ & 0.199 & $1.16 \pm 0.12 \pm 0.22$ \\
$0.22-0.26$ & 0.239 & $0.71 \pm 0.09 \pm 0.07$ \\
$0.26-0.32$ & 0.288 & $0.59 \pm 0.06 \pm 0.07$ \\
$0.32-0.44$ & 0.374 & $0.38 \pm 0.04 \pm 0.03$ \\
$0.44-1.00$ & 0.578 & $0.06 \pm 0.01 \pm 0.01$ \\
\hline
\end{tabular}

Table 40: Measured differential cross section for $K^{* \pm}$ production.

average $K^{* \pm}$ multiplicity per event of $0.62 \pm 0.02 \pm 0.07$ for $x_{E}>0.03$. Using the JETSET Monte Carlo to extrapolate to the full energy range yields $0.71 \pm 0.02($ stat $) \pm 0.08$ (syst $) \pm 0.02($ extr) . The corresponding Monte Carlo numbers are 0.72 for JETSET 7.4, 0.72 for ARIADNE 4.08 and 0.68 for HERWIG 5.8.

\subsubsection{Summary and Discussion}

Most of the data presented in Section 5.2 have been used in Section 2.3 to tune QCD model parameters which control the type of hadrons produced in fragmentation. The parameters are given in Tables 8, 10, 9. Measured multiplicities of identified hadrons and results from the tuned models are summarized in Table 41. 


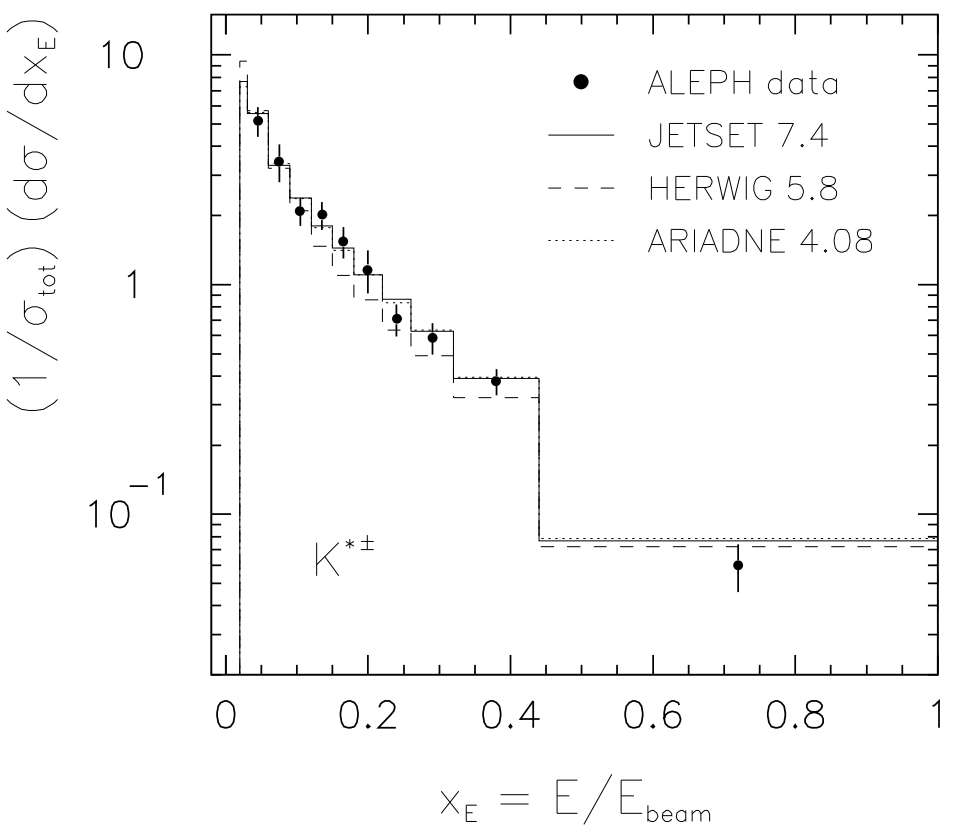

Figure 77: Differential inclusive cross section of $K^{* \pm}$ mesons as function of $x_{E}$.

In the following, parameters of the Lund string model are discussed. The tuned value of the strangeness suppression parameter is $s / u=0.285 \pm 0.015$. It is essentially determined by the rate of strange meson production. The value changes by less than 0.004 if the $K^{0}$ and $K^{+}$ spectra in the fit are restricted to low momenta $(\xi \geq 4)$ where the contribution from heavy flavour ( $c$ and $b$ ) decays is negligible. The probability for the quark and antiquark spins to add up to 1 (which equals $\mathrm{V} /(\mathrm{V}+\mathrm{P})$ if $\mathrm{L}=1$ mesons are absent) is found to be significantly smaller than 0.75 , the value expected from simple spin counting. This is true for both non-strange and strange mesons and suggests that mass effects play an important role. What concerns the $\mathrm{L}=1$ mesons, the ratio of $f_{0}$ to $f_{2}$ production is not consistent with the value $1 / 5$ expected from spin counting.

Ad hoc suppression factors are necessary to describe the rate of $\eta^{\prime}$ production and of baryons at large $\mathrm{x}$ (leading baryon suppression). This latter observation is most striking in the case of the $\Lambda^{0}$ baryon. The rather unnatural popcorn mechanism is needed as well in order to describe baryon production.

As one would expect, owing to the larger number of parameters, JETSET and ARIADNE (both with 15 free parameters) are better than HERWIG (5 free parameters) at describing the multiplicities of identified hadrons. It is interesting that HERWIG is able to predict essentially all of the meson multiplicities. However, the measured baryon rates are not well described by HERWIG even if two more parameters are allowed to vary, the probability to produce di-quark pairs and the probability to produce decuplet baryons in cluster decays. 


\begin{tabular}{|l|c|c|c|c|}
\hline particle & ALEPH data & JETSET 7.4 & ARIADNE 4 & HERWIG 5.8 \\
\hline all charged & $20.91 \pm 0.22$ & 20.65 & 20.60 & 20.63 \\
\hline$\pi^{0}$ & $9.63 \pm 0.64$ & 9.67 & 9.59 & 9.59 \\
$\eta, x \geq 0.1$ & $0.282 \pm 0.022$ & 0.298 & 0.292 & 0.332 \\
$\eta^{\prime}(958), x \geq 0.1$ & $0.064 \pm 0.014$ & 0.071 & 0.071 & 0.081 \\
$K^{0}$ & $2.06 \pm 0.05$ & 2.08 & 2.09 & 2.00 \\
\hline$\rho^{0}(770)$ & $1.45 \pm 0.21$ & 1.37 & 1.37 & 1.33 \\
$\omega(782)$ & $1.07 \pm 0.14$ & 1.29 & 1.28 & 0.86 \\
$\phi(1020)$ & $0.122 \pm 0.009$ & 0.097 & 0.099 & 0.088 \\
$K^{*+}(892)$ & $0.71 \pm 0.08$ & 0.72 & 0.72 & 0.68 \\
$K^{* 0}(892)$ & $0.83 \pm 0.09$ & 0.72 & 0.72 & 0.68 \\
\hline$\Lambda^{0}$ & $0.386 \pm 0.016$ & 0.380 & 0.382 & 0.468 \\
$\Sigma^{0}$ & $0.082 \pm 0.016$ & 0.087 & 0.086 & 0.064 \\
$\Xi^{-}$ & $0.0297 \pm 0.0021$ & 0.0342 & 0.0325 & 0.061 \\
$\Sigma^{ \pm}(1385)$ & $0.065 \pm 0.009$ & 0.068 & 0.068 & 0.164 \\
$\Xi^{0}(1530)$ & $0.0072 \pm 0.0007$ & 0.0068 & 0.0064 & 0.0325 \\
$\Omega^{-}$ & $0.0010 \pm 0.0002$ & 0.0013 & 0.0012 & 0.0098 \\
\hline & DELPHI data & & & \\
$f_{2}(1270), x \geq 0.05$ & $0.17 \pm 0.04$ & 0.16 & 0.16 & 0.16 \\
$f_{0}(980), x \geq 0.05$ & $0.098 \pm 0.016$ & 0.032 & 0.032 & 0 \\
\hline
\end{tabular}

Table 41: Mean multiplicities of identified particles as measured and predicted by several Monte Carlo models. The DELPHI results are taken from [34].

\subsection{Two-Particle Correlations}

The mechanism by which baryons are created in $\mathrm{e}^{+} \mathrm{e}^{-}$annihilations is poorly understood. Single particle spectra can be reproduced by many phenomenological models and are eventually limited in discriminating power. Additional insight into the hadronization mechanism may be sought by considering two-particle correlations.

Information can be gained from the analysis of baryon-antibaryon correlations, like protonantiproton or lambda-antilambda correlations. In two commonly-used phenomenological models, baryon-antibaryon pairs are produced by

(a) the introduction of diquarks as additional partons, as implemented in the JETSET Monte Carlo program; or

(b) the isotropic decay of colourless quark-antiquark clusters of sufficient mass, as implemented in the HERWIG Monte Carlo event generator.

The diquark-antidiquark pair produced by breaking the string in JETSET have compensating transverse momenta. In addition to a simple diquark pair, several breaks in the colour field connecting diquark and antidiquark are permitted (the so called popcorn mechanism [174]), allowing the creation of a meson "between" baryon and antibaryon and reducing any correlation between them. In the default setting of JETSET, $50 \%$ of the baryons are produced by the popcorn mechanism.

The cluster decays in HERWIG cause baryon-antibaryon pairs to be found nearby in phase space, but with compensating transverse momenta. Such correlations are expected to be weaker in JETSET, due to the presence of the popcorn mechanism and because the final momentum 
of the baryon depends on the additional quark needed to accompany the diquark produced in a separate string break.

Additional information is obtained from studies of correlations between strange hadrons, which allow measurement of strangeness suppression factors in the hadron formation mechanism. Finally, the study of Bose-Einstein correlations between identical particles allows measurement of the size of the particle emitting region.

\subsubsection{Proton-Antiproton Correlations}

In this analysis, correlations between protons and antiprotons are studied in rapidity, azimuth and $\cos \theta^{\star}$, the angle between the proton and the sphericity axis in the proton-antiproton rest frame. Hereafter, proton will refer to both protons and antiprotons unless specified otherwise.

\section{Data selection}

Data taken with the ALEPH detector at LEP in 1992 and 1993 were used. A total of 1027801 events was selected with standard event selection requirements. Monte Carlo events were generated with HVFL03 [36] and HERWIG 5.6 and then passed through a simulation of the detector.

Protons were identified by their specific ionization energy loss, $d E / d x$. Tracks were required to have a $d E / d x$ estimated from at least 150 wire measurements. The momentum range $1.35<p<2.35 \mathrm{GeV} / \mathrm{c}$, corresponding to the cross-over of the $d E / d x$ bands for $\mathrm{p}$, $\mathrm{K}$ and $\pi$, was excluded. In order to reduce the contamination due to protons arising from nuclear interactions in the detector material, tracks were required to originate from within a cylinder of radius $0.5 \mathrm{~cm}$ and length $4.0 \mathrm{~cm}$ centred on the interaction point. Given a good $d E / d x$ measurement, $\chi_{i}$ was calculated for the mass hypotheses $i=\mathrm{e}, \pi, \mathrm{K}$ and $\mathrm{p}$ :

$$
\chi_{i}=\frac{(d E / d x)_{m e a s}-(d E / d x)_{i}}{\sigma_{i}} .
$$

A proton was selected if $\left|\chi_{\mathrm{p}}\right|<3$ and $\left|\chi_{\mathrm{e}, \pi, \mathrm{K}}\right|>1.5$. At low momentum the sample is virtually $100 \%$ pure with a $\sim 60 \%$ efficiency, due mainly to the requirement of at least 150 $d E / d x$ measurements. However, there is an excess of protons over antiprotons, due to protons originating from interactions in the material of the detectors. At high momentum, where the mean $d E / d x$ for $\mathrm{K}$ and $\mathrm{p}$ are only $\sim 1 \sigma$ apart, the purity is $\sim 70 \%$ with an efficiency of only $\sim 16 \%$ due to the $\left|\chi_{\mathrm{e}, \pi, \mathrm{K}}\right|$ cut. Although the fragmentation parameters of both JETSET and HERWIG previously had been tuned to reproduce global event-shape and charged-particle inclusive distributions [26], some difference in purity was observed using these models separately. This arose from the different hadron fractions in the two models.

The numbers of unlike-sign and like-sign pairs selected in the data were 22177 and 11820 respectively. Events in which exactly two protons were found were accepted. The oppositelycharged pair sample contains misidentified hadrons and protons and antiprotons arising from different baryon-antibaryon pairs. Monte Carlo studies show that this background is well reproduced by the distribution of like-sign pairs. These have been subtracted in the corrected distributions.

Biases resulting from the $d E / d x$ selection, from jet reconstruction and from the backgrounds (arising from misidentification and from protons created in nuclear interactions in the material of the detector) were investigated and found to be small. 


\section{Correlation Studies}

Using the selected proton pairs, correlations have been studied in the following variables: rapidity $y$, azimuth $\varphi$ and $\cos \theta^{\star}$ (the angle between proton and sphericity axis in the protonantiproton rest frame). The distributions were corrected with a sample of JETSET 7.3 events generated without initial state radiation and with the default setting of $50 \%$ popcorn production of baryons. $\Lambda$ and other weakly-decaying baryons as well as $K_{s}^{0}$ were required to decay. The baryons remaining are thus protons and neutrons. In comparisons of data with Monte Carlo simulations, parameters relating to baryon production were fixed at their default settings unless specified otherwise.

The rapidity $y$ is calculated with respect to the sphericity axis, assuming the proton mass for each track

$$
y=\frac{1}{2} \ln \left(\frac{E+p_{L}}{E-p_{L}}\right) .
$$

Figure 78 shows the corrected like-sign subtracted rapidity distribution for antiprotons, given a tagging proton in five ranges of $y$. Since the primary interest is in the strength of any correlation, the histograms have been normalized to unit area. There is a clear local compensation of baryon number. The strength of this correlation in JETSET is in fair agreement with the data, while HERWIG predicts narrower distributions.

Hadrons nearby in rapidity may receive a common sideways boost in multi-jet events, leading to a correlation in azimuthal angle $\varphi$. In order to investigate this possibility, the distribution in $\varphi_{\text {jet }}$ about the jet axis has been measured.

Jets were constructed using the Durham algorithm with $y_{\text {cut }}=0.0025$. This results in rates of $23 \%, 39 \%$ and $38 \%$ for events with two, three and four or more jets, respectively. The azimuth $\varphi_{\text {jet }}$ was measured in the plane perpendicular to the jet axis for proton pairs in which both particles were associated with the same jet. The axes are chosen so that $\varphi_{\text {jet }}=0$ points out of the event plane, as defined by the sphericity tensor major and semi-major axes. Figure 79 shows the like-sign subtracted corrected $\varphi_{\text {jet }}$ distribution compared with JETSET and HERWIG and with random unlike-sign pairs. The histograms are normalized to unit area. The data show a slight enhancement at $\Delta \varphi_{\text {jet }}=\pi$, when the tagging proton is out of the event plane, which is well reproduced by JETSET. HERWIG peaks more prominently at $\pi$, indicating a stronger transverse momentum compensation. Varying the popcorn fraction in JETSET does not result in significant changes in the length of correlations in azimuth.

The angle between proton and sphericity axis in the proton-antiproton rest frame, $\theta^{\star}$, was first used to discriminate between baryon production models by the TPC/2 $\gamma$ collaboration [175]. Proton-antiproton pairs arising from the isotropic decay of a mesonic quark cluster have a flat distribution in $\cos \theta^{\star}$, whereas in a string-like mechanism the momentum difference is expected, on average, to lie along the string direction. Figure 80 shows the like-sign subtracted, corrected $\left|\cos \theta^{\star}\right|$ distribution, compared with the predictions of HERWIG and JETSET. The histograms are normalized to unit area. HERWIG, in which protons arise from the decay of colourless quark-antiquark clusters, has a flat distribution. The data, however, peak at $\left|\cos \theta^{\star}\right|=1$, and are well reproduced by JETSET. The distribution of randomly-chosen unlike-pairs peaks much more narrowly at $\left|\cos \theta^{\star}\right|=1$ than do proton-antiproton pairs.

In summary, proton-antiproton correlations in hadronic Z decays have been studied in rapidity, azimuth and $\cos \theta^{\star}$, the angle between proton and sphericity axis in the protonantiproton rest frame. A strong local compensation of baryon number in rapidity is observed: given a tagging proton, $70 \%$ of the excess of additional antiprotons over additional protons is 


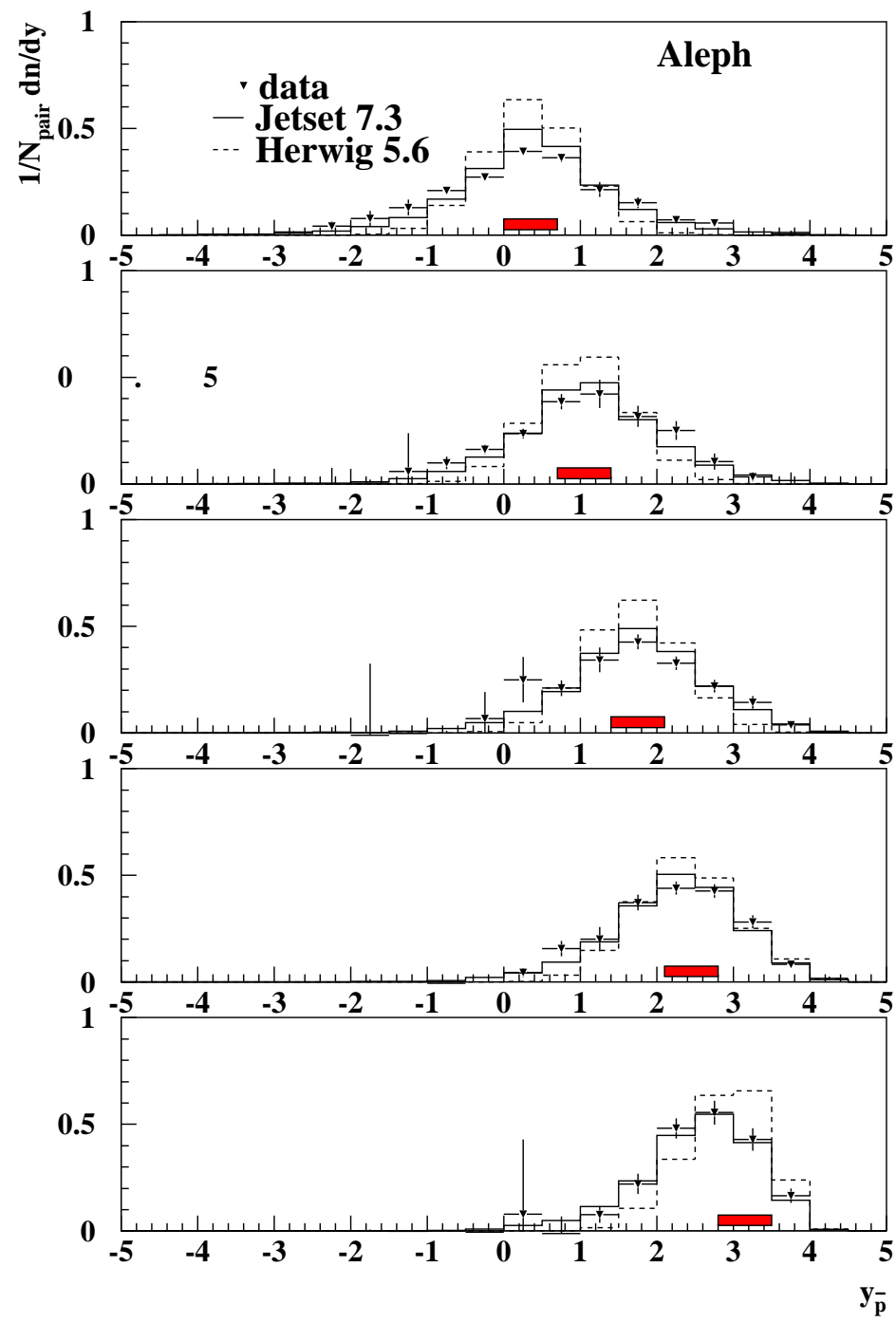

Figure 78: Like-sign subtracted rapidity distribution of antiprotons, given a tagging proton in the shaded region. The errors shown are statistical. Superimposed are the predictions of JETSET and HERWIG. The histograms are normalized to unit area. Negative values, of negligible contribution, are set to zero.

found within one unit of rapidity from the proton. With the parameters relating to baryon production fixed at their default settings, a shorter correlation length is predicted by both JETSET and HERWIG. No evidence for an anticorrelation in azimuth is seen. The data are well reproduced by JETSET, while HERWIG predicts an anticorrelation in azimuth. Both data and JETSET peak at $\left|\cos \theta^{\star}\right|=1$, while the cluster decays in HERWIG give a flat distribution.

\subsubsection{Strangeness Correlations}

ALEPH has studied correlations between $K_{S}^{0}$ mesons and $\Lambda$ baryons using the same selection as for the inclusive analysis [171]. The two-particle correlation as a function of rapidity is defined as:

$$
C\left(y_{a}, y_{b}\right)=N_{\text {had }} \frac{n\left(y_{a}, y_{b}\right)}{n\left(y_{a}\right) n\left(y_{b}\right)}
$$

where $y$ is the rapidity along the thrust-axis. $N_{\text {had }}$ is the number of hadronic events considered, $n\left(y_{a}, y_{b}\right)$ is the density of particle pairs with one particle at rapidity $y_{a}$ and the other one at $y_{b}$, and $n(y)$ is the single particle density. The corrected results are shown in Fig. 81 as a function of $y_{a}$ for two choices of $y_{b}: 0.5<y_{b}<1.5$ and $2.5<y_{b}<3.5$. 


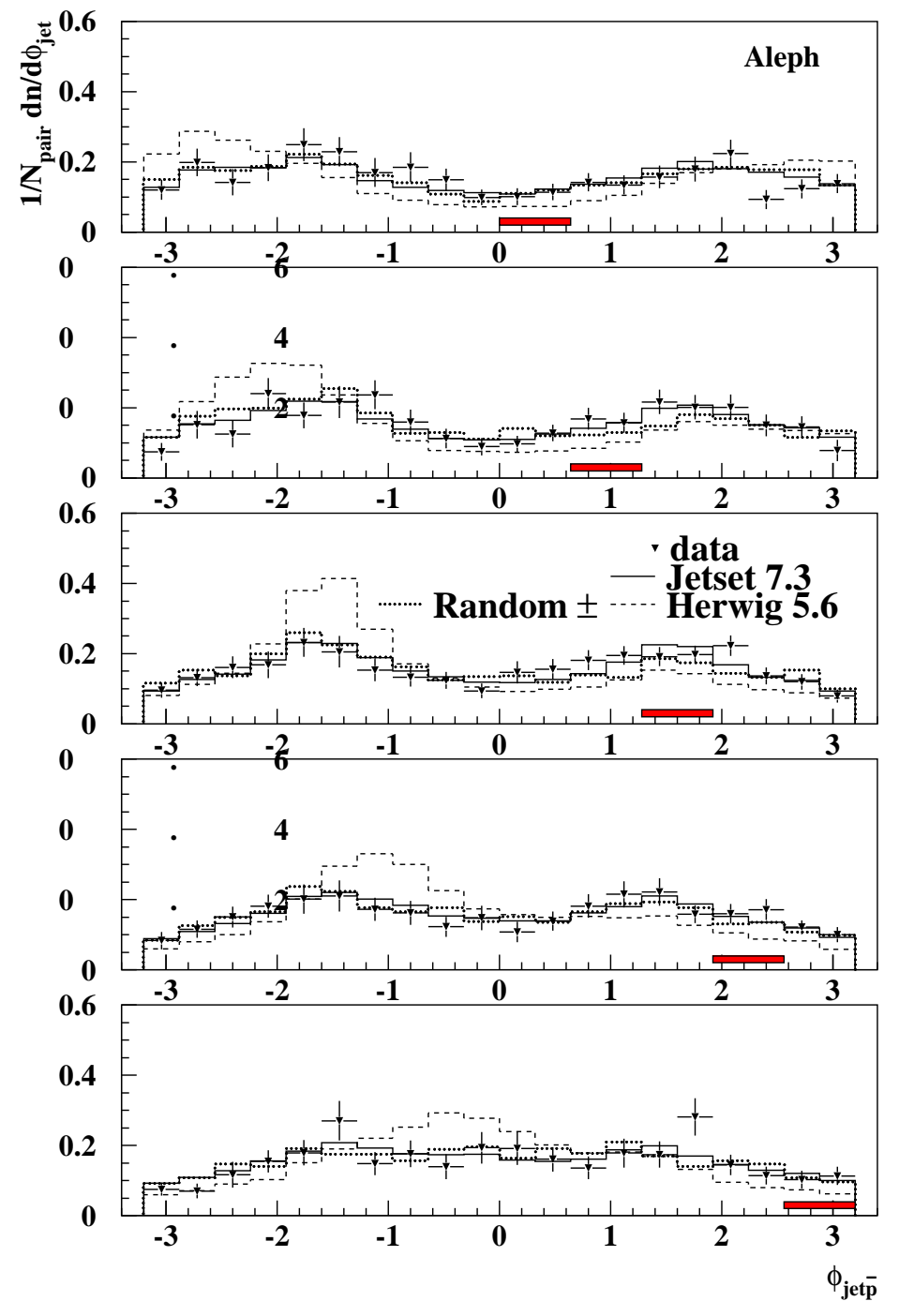

Figure 79: Like-sign subtracted, corrected $\varphi_{\text {jet }}$ distribution of antiprotons, given a tagging proton in the shaded region. $\varphi_{\text {jet }}$ is measured in the plane perpendicular to the jet axis. The errors shown are statistical. Superimposed are the predictions of JETSET and HERWIG and the distribution for randomly-chosen unlike-sign pairs taken from JETSET. The histograms are normalized to unit area.

The main features of the rapidity correlations seen in Fig. 81 are a strong short range correlation for $\Lambda \bar{\Lambda}$, a weaker one for $K_{S}^{0} K_{S}^{0}$ and $\Lambda K_{S}^{0}$ and a short range anti-correlation for $\Lambda \Lambda$. These structures are all well reproduced by JETSET, whereas HERWIG overestimates the $\Lambda \bar{\Lambda}$ correlation by a factor of two.

Figure 82 shows the correlation function projected along rapidity difference. This distribution is obtained by dividing the distribution of rapidity differences by that of two particles taken from different events. The denominator is normalized to the number of pairs expected in case of no correlations.

Also shown in Fig. 82 are the correlation functions predicted by JETSET for various popcorn parameters and by HERWIG. The $\Lambda \bar{\Lambda}$ data are found consistent with JETSET with the standard popcorn probability of $50 \%$ and less consistent with the option having no popcorn mechanism. The systematic errors of the measurement prevent more quantitative statements.

Since the average $\Lambda \bar{\Lambda}$ and $\Lambda \Lambda$ pair multiplicity is also quite sensitive to the popcorn parameter, it can be used as a consistency check. Table 42 compares the measured two-particle multiplicities with model predictions. The columns labeled "Uncorr." contain the expectation if the particles were produced uncorrelated, obtained from the single particle multiplicities. The predicted multiplicity of $\Lambda$ pairs decreases approximately linearly with the popcorn probability. 


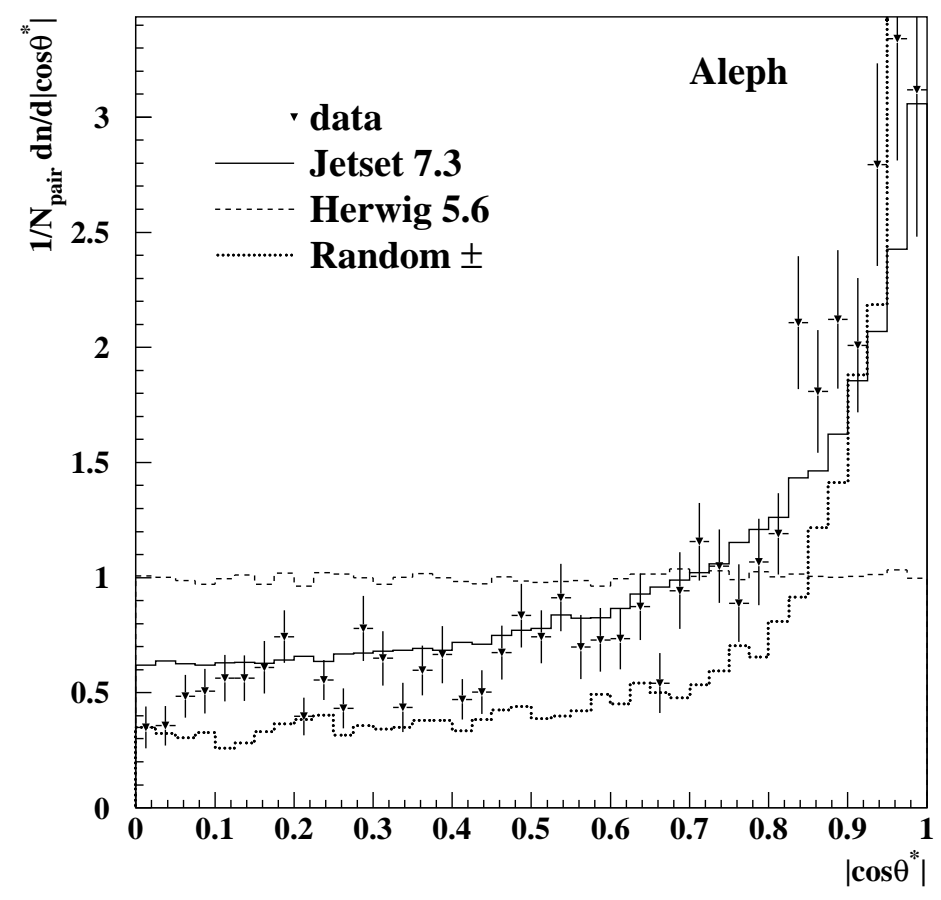

Figure 80: Like-sign subtracted, corrected $\left|\cos \theta^{\star}\right|$ distribution of protonantiproton pairs. $\theta^{\star}$ is the angle between proton and sphericity axis in the protonantiproton rest frame. The errors shown are statistical. Superimposed are the predictions of JETSET and HERWIG and the distribution for randomly-chosen unlike-sign pairs taken from JETSET. The histograms are normalized to unit area.

\begin{tabular}{|c||cc|c||c|c||c|c|}
\hline$\langle n\rangle$ & \multicolumn{2}{|c|}{ ALEPH Data } & Uncorr. & JETSET & Uncorr. & HERWIG & Uncorr. \\
\hline$\Lambda \bar{\Lambda}$ & $0.093 \pm 0.009$ & 0.037 & 0.092 & 0.039 & 0.192 & 0.050 \\
$\Lambda \Lambda+\bar{\Lambda} \bar{\Lambda}$ & $0.028 \pm 0.003$ & 0.037 & 0.031 & 0.039 & 0.048 & 0.050 \\
$\Lambda K_{S}^{0}+\bar{\Lambda} K_{S}^{0}$ & $0.403 \pm 0.029$ & 0.397 & 0.427 & 0.377 & 0.474 & 0.491 \\
$K_{S}^{0} K_{S}^{0}$ & $0.593 \pm 0.036$ & 0.531 & 0.619 & 0.557 & 0.695 & 0.601 \\
\hline
\end{tabular}

Table 42: Average multiplicities of $\Lambda$ and $K^{0}$ pairs compared with model predictions. The columns labeled Uncorr contain the expectation if the particles were produced uncorrelated.

The measured multiplicity thus constrains the popcorn probability to be within the range $0.50 \pm 0.10$ (assuming this to be the only free parameter affecting the $\Lambda$ pair multiplicity). In conclusion, the data gives no reason to change the default $50 \%$ popcorn probability in JETSET.

In the following, the particle pairs are restricted to the interval $\Delta y<1.5$ and the structure of the short range correlation is studied as a function of other variables. The same procedure as used for rapidity differences is used to find the correlation as a function of $\Delta \phi$, where $\phi$ is the azimuthal angle around the thrust axis. This is shown in Fig. 83.

For $\Lambda \bar{\Lambda}$ pairs, a large peak is seen at $\Delta \phi=0^{\circ}$, and no significant peak is seen at $\Delta \phi=180^{\circ}$. Hence, there is no hint of local $p_{T}$ compensation among correlated $\Lambda \bar{\Lambda}$ pairs. This is in contrast to observations at centre-of-mass energies around $10 \mathrm{GeV}$, where baryon-antibaryon pairs are predominantly back-to-back in azimuth [176]. At PETRA energies there is an intermediate situation with no prominent peaks neither at $\Delta \phi=0^{\circ}$ nor at $\Delta \phi=180^{\circ}$ [164]. For the other two-particle combinations, both a same-side and a back-to-back correlation is seen. All of these features are well reproduced by JETSET, and not so well by HERWIG which predicts a strong back-to-back correlation in the case of $\Lambda \bar{\Lambda}$ pairs. The predicted correlations do not 

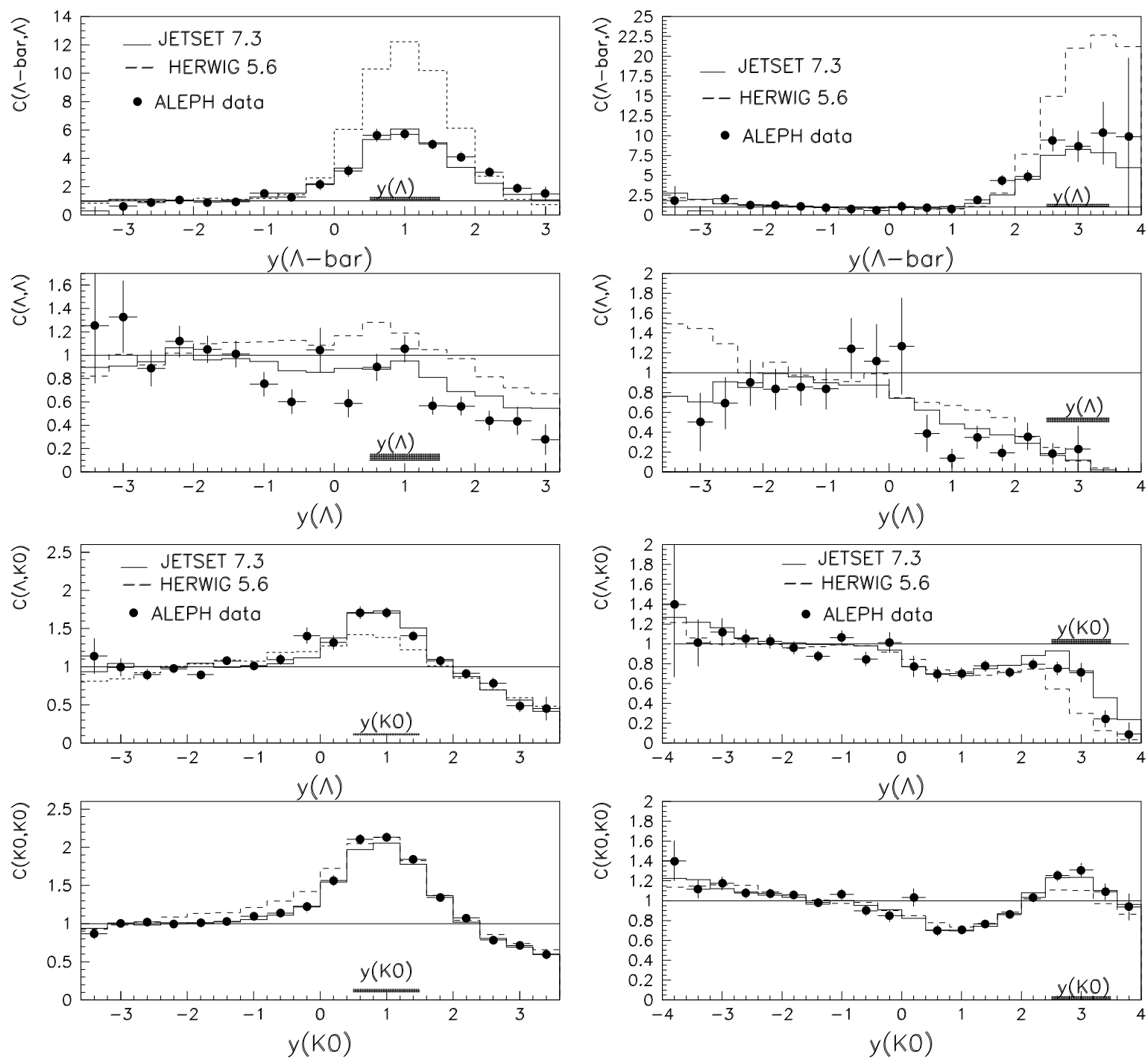

Figure 81: Two-particle $\Lambda \bar{\Lambda}, \Lambda \Lambda$ and $\Lambda K_{S}^{0}, K_{S}^{0} K_{S}^{0}$ correlations as a function of $y_{a}$ for two $y_{b}$ intervals.

depend significantly on the popcorn probability.

Another interesting variable is the polar angle of the $\Lambda \bar{\Lambda}$ pair in its rest frame relative to the thrust axis. The normalized angular distribution shown in Fig. 84 shows an alignment of the $\Lambda \bar{\Lambda}$ pair along the thrust axis. In JETSET the baryon pairs are aligned along the parent string, and this model reproduces data. The HERWIG clusters, on the other hand, decay isotropically. A version of HERWIG introducing an anisotropy by letting clusters containing a quark from the perturbative shower decay in the direction of this quark [177] is also shown in Fig. 84, but this is apparently not enough to describe the data.

In summary, the correlations as a function of rapidity and azimuth relative to the thrust axis within pairs of $\Lambda$ and $K^{0}$ are found to be in good agreement with JETSET. Also HERWIG reproduces many of the features. However, the $\Lambda \bar{\Lambda}$ correlation disagrees with HERWIG, both in size and in qualitative features. The observed correlation length in $\Lambda \bar{\Lambda}$ indicates that some amount of the "popcorn" mechanism in JETSET is necessary to describe the data. 



Figure 82: Two-particle correlations as a function of rapidity difference.
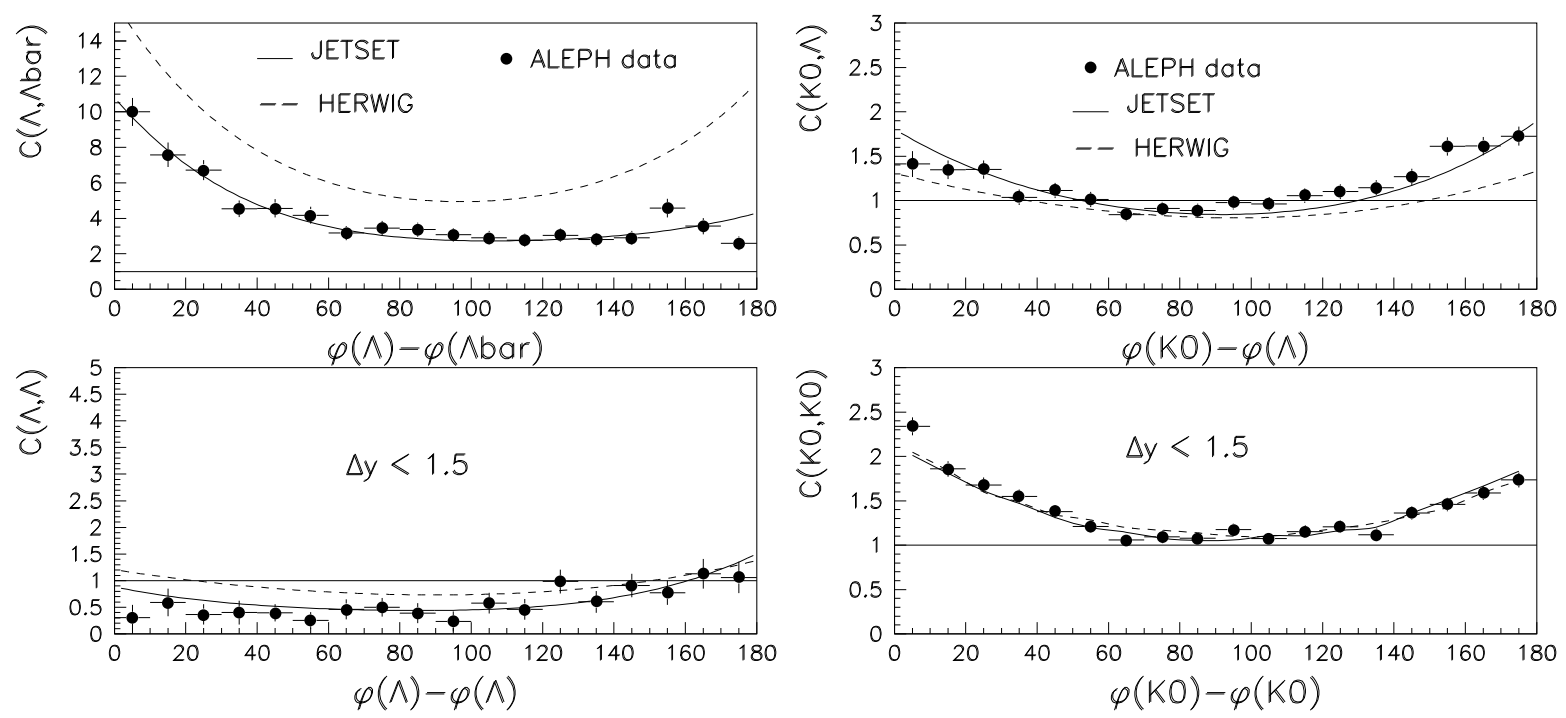

Figure 83: Two-particle correlations as a function of azimuthal separation, for $|\Delta y|<1.5$.

\subsubsection{Bose-Einstein Correlations}

As a consequence of Bose-Einstein (BE) statistics, interference effects are expected between identical bosons which are emitted close to each other in phase space. The BE correlations enhance the two particle differential cross section for like-sign charged pions compared to unlikesign charged pions when the two particles have similar momenta [178]. A similar enhancement is observed for pairs of $K_{S}^{0}$ mesons [171].

For a pair of identical bosons(fermions), the quantum mechanical wave-function must be symmetric(antisymmetric) under particle exchange. This requirement alters the twoparticle differential cross section for the production of identical particles from a source, whose 


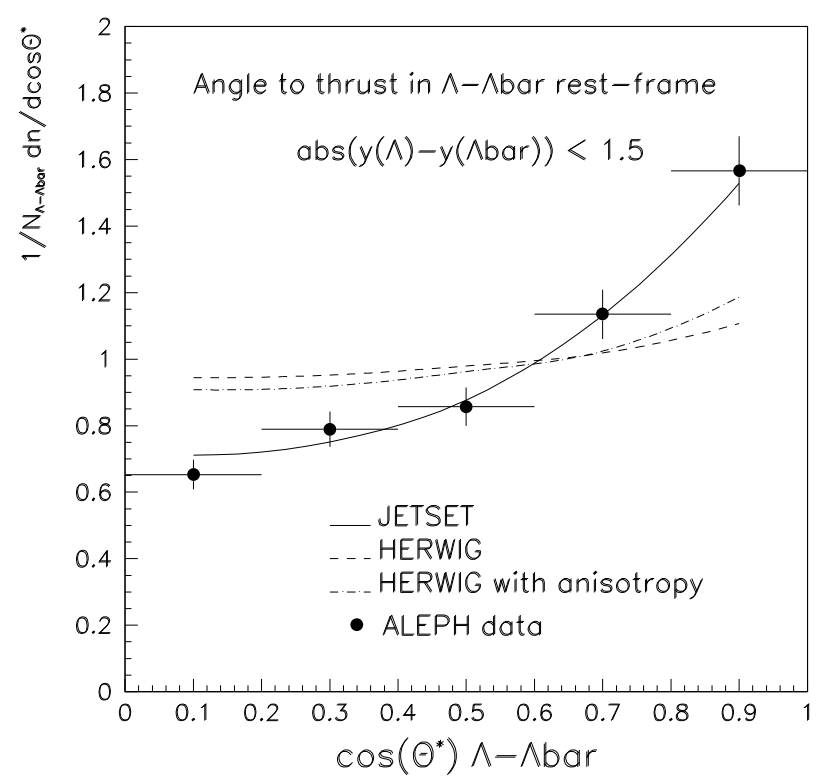

Figure 84: Angle of $\Lambda$ to thrust axis in the $\Lambda \bar{\Lambda}$ rest frame, for $|\Delta y|<1.5$.

distribution in space-time $x$ is given by $\rho(x)$, by a factor $[179,180,181]$

$$
R(\Delta p)=1 \pm \lambda|\tilde{\rho}(\Delta p)|^{2}
$$

where the $+(-)$ sign applies if the spatial wave-function of the particles is symmetric(antisymmetric) under particle exchange. The parameter $\lambda$ lies in the range zero to one, being zero for a completely incoherent source and one for a completely coherent one. The four-vector $\Delta p$ is the difference in the four-momenta of the two particles and $\tilde{\rho}(\Delta p)$ is the four-dimensional Fourier transform of $\rho(x)$.

As a pair of identical pions must have a symmetric spatial wave-function, it follows from Eq. (85) that their two-particle differential cross section is enhanced by a factor which tends towards a maximum of $(1+\lambda)$ for pions of identical four-momenta.

Describing the source by a spherically symmetric Gaussian distribution of width $\sigma$, the expected Bose-Einstein enhancement becomes

$$
R(Q)=1+\lambda \exp \left(-Q^{2} \sigma^{2}\right)
$$

where $Q=\sqrt{\left(\mathbf{p}_{1}-\mathbf{p}_{2}\right)^{2}-\left(E_{1}-E_{2}\right)^{2}}$ is the Lorentz invariant momentum difference of the two pions.

In the ALEPH charged pion analysis [178] two different reference samples are used. The first sample consists of pairs of unlike-charged pions. An approximation to $R(Q)$ is then given by

$$
r_{+-}(Q)=\frac{N_{++}(Q)}{N_{+-}(Q)}
$$

where $N_{++}(Q)$ and $N_{+-}(Q)$ are the number of like and unlike-charged pairs as a function of $Q$. The second method of obtaining a reference sample uses the technique of event mixing. Pairs of pions are formed by combining a pion from the event under study with a pion from a previous event. The momentum vector of each of these pions is measured with respect to a coordinate system defined by the eigenvectors of the sphericity tensor of the event from which it came. If all events comprised two back to back jets moving parallel to the sphericity axis, 
then apart from the lack of Bose-Einstein correlations, the differential cross section for these 'event-mixed' pairs would be very similar to that of the like-charged pairs. $R(Q)$ can then be approximated by

$$
r_{m i x}(Q)=\frac{N_{++}(Q)}{N_{m i x}(Q)}
$$

where $N_{\text {mix }}(Q)$ is the number of event-mixed pairs as a function of $Q$.

The data are corrected for Coulomb repulsion/attraction at small $Q$ by applying a Gamow factor [181]. The uncertainty of this correction (see [182]) is included in the systematic error.

Imperfections in the reference samples are taken into account by dividing the data ratio by the corresponding Monte Carlo prediction:

$$
R_{+-}(Q)=r_{+-}^{\text {data }}(Q) / r_{+-}^{M C}(Q)=\frac{N_{++}^{\text {data }}(Q)}{N_{+-}^{\text {data }}(Q)} / \frac{N_{++}^{M C}(Q)}{N_{+-}^{M C}(Q)}
$$

and

$$
R_{m i x}(Q)=r_{m i x}^{\text {data }}(Q) / r_{m i x}^{M C}(Q)=\frac{N_{++}^{\text {data }}(Q)}{N_{m i x}^{\text {data }}(Q)} / \frac{N_{++}^{M C}(Q)}{N_{m i x}^{M C}(Q)}
$$

where the Monte Carlo simulation ( JETSET 6.3) does not include Bose-Einstein correlations. After correction for the pion purity the resulting double ratios $R_{+-}(Q)$ and $R_{m i x}(Q)$ are plotted in Figs. 85(a) and (b), respectively. The curve superimposed over each of Figs. 85 (a), (b) is a fit of the form

$$
R_{+-}(Q), R_{m i x}(Q)=\kappa(1+\epsilon Q)\left[1+\lambda \exp \left(-Q^{2} \sigma^{2}\right)\right]
$$

which is Eq. (86) multiplied by a linear function in $Q$ to try to take into account imperfections in the Monte Carlo simulation. When fitting $R_{+-}(Q)$, the regions $0.388<Q<0.436 \mathrm{GeV}$ and $0.502<Q<0.932 \mathrm{GeV}$ are excluded to remove sensitivity to the production rates of $K^{0}$ 's and $\rho^{0}$ s.

The differences between the two reference samples are probably due to the inadequate Monte Carlo simulation. It does not simulate final state strong interactions and it overproduces $\eta^{\prime}$. However, other effects may also be contributing. Therefore rather large systematic uncertainties are given in the final result: $\lambda=0.51 \pm 0.04 \pm 0.11$ and $\sigma=3.3 \pm 0.2 \pm 0.8 \mathrm{GeV}^{-1}$. This corresponds to a spherically symmetric source with an r.m.s. radius of $\sigma=0.65 \pm 0.04 \pm 0.16 \mathrm{fm}$. This value is comparable with those obtained at lower energy $e^{+} e^{-}$colliders $[183,184,185,186]$.

The measured value for the chaoticity parameter $\lambda$ must be corrected for resonance decays. Pion pairs in which one pion comes from the decay of a narrow resonance or a weakly decaying particle and the other pion comes from elsewhere will not contribute to the enhancement at small $Q$. Enabling Bose-Einstein correlations in the JETSET 7.3 simulation, a Monte Carlo chaoticity parameter of $\lambda_{M C}=2.1 \pm 0.1 \pm 0.1$ is required to describe the two pion mass spectrum [170]. This value is even larger than the expectation $\lambda=1$ for a completely coherent source.

Bose-Einstein correlations are also observed in the $K_{S}^{0} K_{S}^{0}$ system [171]. For pairs of neutral mesons there are no Coulomb correction factors, however, there is also no data reference sample of like-charged particles. The two neutral kaons either come from $K^{0} K^{0}$ or $\bar{K}^{0} \bar{K}^{0}$ decays which are identical bosons or from $K^{0} \bar{K}^{0}$ decays in which case charge conjugation invariance predicts a BE-like enhancement for the $K_{S}^{0} K_{S}^{0}$ pairs [187].

Figure 86 shows the ALEPH data as a function of $Q=\sqrt{M_{K K}^{2}-4 m_{K}^{2}}$. The corrected $Q$ distribution of the data is divided by the Monte Carlo prediction of JETSET 7.3 without BE correlation. The ratio is normalized in the interval between $0.6 \mathrm{GeV}$ and $2.5 \mathrm{GeV}$ (excluding 



Figure 85: $R_{+-}(Q)=r_{+-}^{\text {data }}(Q) / r_{+-}^{M C}($ top $)$ and $R_{m i x}(Q)=r_{m i x}^{\text {data }} / r_{\text {mix }}^{M C}$ (bottom), corrected for non-pion background. The curves represent fits according to Eq. (87).

the range $1.1 \mathrm{GeV}$ to $1.5 \mathrm{GeV}$ where resonances such as $f_{2}^{\prime}(1525)$ and $f_{0}(1710)$ may contribute). The ratio is fitted to Eq. (87).

Correcting for the $f^{0}(975)$ meson as described in reference [188], the source size is found to be $\sigma=0.65 \pm 0.07 \pm 0.15 \mathrm{fm}$ and the chaoticity parameter $\lambda=1.0 \pm 0.3 \pm 0.4$. The major systematic uncertainties are the $f^{0}$ width and rate and the composition of the reference sample. Given the rather large errors, this result for $K_{S}^{0} K_{S}^{0}$ pairs is in agreement with the result for $\pi^{+} \pi^{-}$pairs. 




Figure 86: The experimental $Q\left(K_{S}^{0} K_{S}^{0}\right)$ distribution divided by the Monte Carlo distribution for a generator without BoseEinstein correlation. 


\section{Summary}

With the high statistics data collected in hadronic $Z$ decays significant progress in the understanding of the dynamics of QCD has been achieved, spanning the hard perturbative regime, the parton showering process and the non-perturbative region of hadron formation.

The strong coupling constant $\alpha_{s}$ has been measured from $R_{\tau}$ at the scale of the tau mass, from scaling violations in fragmentation functions between $\sqrt{s}=22 \mathrm{GeV}$ and $\sqrt{s}=M_{Z}$, and from the analysis of global event shape variables and $R_{l}$ at the scale of the $Z$ mass. The energy evolution is consistent with the running expected from QCD.

Testing the structure of QCD, it has been confirmed that quarks have spin $1 / 2$ and that gluons have spin 1. The non-Abelian nature of QCD requires the strong coupling constant to be flavour independent, consistent with the experimental findings for the ratios $\alpha_{s}(b) / \alpha_{s}(u d s c)$ and $\alpha_{s}(u d s) / \alpha_{s}(b c)$. Information about the gauge structure of strong interactions has been obtained from measurements of the colour factors from the study of kinematical correlations in multi-jet events and the running of the strong coupling constant between the scale of the $\tau$ and the $\mathrm{Z}$ mass. Combining all information, the ALEPH results for the colour factor ratios show that the gauge structure of QCD is compatible with the expectation for an unbroken SU(3) symmetry, while it is incompatible with the predictions based on many other gauge groups, including any Abelian model.

Experimental evidence was shown that the parton showering process proceeds like in a self-similar branching process as implemented in coherent parton shower models. The importance of coherence effects is demonstrated by the energy evolution of inclusive momentum spectra and charged particle multiplicities, both of which are quantitatively described by the modified leading-log approximation (MLLA) and local parton-hadron duality (LPHD), and by comparing measured particle-particle correlation functions with predictions from various Monte Carlo models. Studies of the string effect show that colour coherence is present both in the perturbative and the non-perturbative phase of hadronic $\mathrm{Z}$ decays.

The high statistics data available at LEP for the first time facilitated detailed comparisons between quark and gluon jets in three jet events, showing differences which result from the different colour charges of quarks and gluons. The gluon jet was found to be wider than an equivalent quark jet with higher multiplicities and a softer momentum spectrum. However, the differences observed at the level of the final state hadrons were much smaller than the expectations for asymptotic energies, which is understood to be at least partly caused by colour coherence effects. Another sensitive probe of quark/gluon differences is the study of subjet multiplicities, which, looking closer to the hard perturbative regime, independently confirmed the different colour charges of the two kinds of partons.

Prompt photon production in hadronic events provides a unique window for looking into the early stages on the parton showering process free from fragmentation effects. The studies with isolated photons in hadronic events have shown inadequacies in the treatment of photon radiation during the parton showering process in all models studied. Measuring prompt photons also inside jets has allowed the first determination of the quark-to-photon fragmentation function. Using this information, the description of the data has improved substantially.

The detailed understanding of the hadronization stage still relies on phenomenological models, which, however, are quite successful in describing the relative production rates and momentum spectra of the final state particles. Measurements were presented for identified pions, kaons and protons, strange mesons and baryons, vector mesons and single photons. More detailed information about the dynamics of hadron formation was obtained from the 
study of two-particle correlations. It was found that the JETSET and ARIADNE string models successfully parametrize two-particle correlations related to strangeness and baryon number. Most of the parameters in JETSET and ARIADNE have a rather natural physical meaning, in that they correspond to suppression effects for heavier particles, which are difficult to predict from first principles. The baryon spectra, however, require at least two rather unnatural parameters, the so-called leading baryon suppression and popcorn; this casts some doubt on the validity of the baryon production scheme via diquarks. HERWIG, with far fewer parameters, is able to predict essentially all of the meson multiplicities, while it reproduces far less well the baryon rates. Finally, the source size, or equivalently the hadronization scale, was measured by means of Bose-Einstein correlations to be around $0.65 \mathrm{fm}$.

This paper has summarized the ALEPH results on QCD based on most of the LEP I data, taken between 1989 and 1995. The higher centre-of-mass energies available at LEP II, which started in November 1995, as well as the development of new theoretical and experimental techniques, will allow for additional tests of QCD in the future. 


\section{Appendix}

\section{A $\quad R_{l}$ and $R_{\tau}$ for Arbitrary Colour Factors}

This section contains a compilation of the ingredients that go into the theoretical prediction for $R_{l}$ and $R \tau$ for arbitrary colour factors. Although the QCD (SU(3)) expressions are well known, the ones for the general case that the dynamics of strong interactions is described by any unbroken gauge symmetry based on a simple Lie group, i.e. a theory with one universal coupling constant and massless gluons, are rather scattered through the literature.

It is convenient to redefine the coupling constant such that the amplitude for gluon emission from a quark is independent of the gauge group of the theory. Also absorbing a factor of $2 \pi$ yields

$$
a=\frac{\alpha_{s} C_{F}}{2 \pi} .
$$

The predictions of the theory for $n_{F}$ quark degrees of freedom then can be expressed as function of the free parameter $a$ and the variables

$$
x=\frac{C_{A}}{C_{F}}, y=\frac{T_{F}}{C_{F}} \quad \text { and } \quad z=y \cdot n_{f} .
$$

All expressions apply for the $\overline{\mathrm{MS}}$ renormalization scheme.

\section{A.1 The Running Coupling Constant and Masses}

The variation of the strong coupling constant $a$ and renormalized masses $\bar{m}$ with the renormalization scale of the theory is described by a coupled system of differential equations:

$$
\begin{aligned}
\frac{d a}{d \ln \mu} & =-b_{0} a^{2}-b_{1} a^{3}-b_{2} a^{4} \ldots \\
\frac{d \ln \bar{m}}{d \ln \mu} & =-g_{0} a-g_{1} a^{2} \ldots
\end{aligned}
$$

The coefficients $b_{i}$ and $g_{i}$ depend on the specific theory, with the leading coefficients given by $[189,190,103,191]$ :

$$
\begin{aligned}
& b_{0}=\frac{11}{3} x-\frac{4}{3} z \\
& b_{1}=\frac{17}{3} x^{2}-\frac{10}{3} x z-2 z \\
& b_{2}=\frac{2857}{216} x^{3}-\frac{1415}{108} x^{2} z+\frac{158}{108} x z^{2}-\frac{205}{36} x z+\frac{11}{9} z^{2}+\frac{1}{2} z \\
& g_{0}=3 \\
& g_{1}=\frac{3}{4}+\frac{97}{12} x-\frac{5}{3} z
\end{aligned}
$$

Equation (88) determines how the strong coupling constant evolves for a fixed number of active flavours. The treatment of flavour thresholds is described in [192]. It turns out that the 
actual value of the scale $\mu$ where a flavour threshold occurs can be arbitrary, as long as the proper matching condition between $n_{f}$ and the $n_{f} \pm 1$ is chosen,

$$
a\left(n_{f} \mp 1\right)=a\left(n_{f}\right) \pm a^{2}\left(n_{f}\right) \frac{4}{3} y L+a^{3}\left(n_{f}\right)\left[\left(\frac{4}{3} y L\right)^{2} \pm\left(\frac{10}{3} x y+2 y\right) L \pm\left(\frac{8}{9} x y-\frac{17}{12} y\right)\right]
$$

with $L=\ln (\bar{m}(\bar{m}) / \mu)$. Thus $a$, in general, will be discontinuous at flavour thresholds. For convenience the threshold $\mu$ may be chosen such that $a$ is continuous. To the order given above, this is achieved for

$$
\mu=\frac{M}{1+k a(M)} \quad \text { with } \quad k=2+\left(\frac{17}{16}-\frac{2}{3} x y\right)
$$

where $M$ is the pole mass of a quark. To leading order $M$ is related to the running mass according to

$$
\bar{m}(M)=\frac{M}{1+2 a(M)} .
$$

With those ingredients, a consistent treatment of running masses and coupling constants when starting at the scale $M_{Z}$ is achieved by the following procedure: one starts evolving down to the scale of the $b$ quark mass $M_{b}$ with $n_{f}=5$, which gives $a\left(M_{b}\right)$. Then the value for the flavour threshold $\mu_{b}$ and the running $b$ mass $\bar{m}_{b}(M)$ is determined according to Eq. (92) and Eq. (93), respectively. The five-flavour evolution then continues until $\mu_{b}$, where the continuous transition is made to $n_{f}=4$. Iterating this scheme, the strong coupling and the running masses for all flavours can be determined for any scale.

A final remark is in order for the determination of $a\left(M_{\tau}\right)$. Because the tau lepton mass $M_{\tau}$ is larger than the $c$ quark mass, while the number of active flavours is only $n_{f}=3$, the evolution first has to be run down to the $c$ threshold keeping $n_{f}=4$ and then up again to $M_{\tau}$ using $n_{f}=3$.

\section{A.2 Theoretical Predictions for $R_{\gamma}$}

The QCD corrections both for $R_{\tau}$ and $R_{l}$ are related to the QCD correction $\delta^{0}$ of $R_{\gamma}$

$$
R_{\gamma}=\frac{\sigma\left(e^{+} e^{-} \rightarrow \gamma \rightarrow \text { hadrons }\right)}{\sigma_{\text {Born }}\left(e^{+} e^{-} \rightarrow \gamma \rightarrow \mu^{+} \mu^{-}\right)}=3 \sum_{f} q_{f}^{2}\left(1+\delta^{0}\right)
$$

which is known to order $a^{3}[43]$ :

$$
\delta^{0}=K_{1} a+K_{2} a^{2}+\left(K_{3}+R_{3}+T_{3} \frac{\left(\sum_{f} q_{f}\right)^{2}}{3 \sum_{f} q_{f}^{2}}\right) a^{3}
$$

For the strong coupling constant taken at the centre-of-mass energy of the hadronic system the coefficients are

$$
\begin{aligned}
& K_{1}=\frac{3}{2} \\
& K_{2}=-\frac{3}{8}+x\left(\frac{123}{8}-11 \zeta_{3}\right)-z\left(\frac{11}{2}-4 \zeta_{3}\right)
\end{aligned}
$$




$$
\begin{aligned}
K_{3}= & -\frac{69}{16}-x\left(\frac{127}{8}+\frac{143}{2} \zeta_{3}-110 \zeta_{5}\right)+x^{2}\left(\frac{90445}{432}-\frac{2737}{18} \zeta_{3}-\frac{55}{3} \zeta_{5}\right) \\
& -z\left(\frac{29}{8}-38 \zeta_{3}+40 \zeta_{5}\right)-x z\left(\frac{3880}{27}-\frac{896}{9} \zeta_{3}-\frac{20}{3} \zeta_{5}\right)+z^{2}\left(\frac{604}{27}-\frac{152}{9} \zeta_{3}\right) \\
R_{3}= & -\frac{\pi^{2}}{8}\left(\frac{11}{3} x-\frac{4}{3} z\right)^{2} \\
T_{3}= & \frac{d^{a b c} d^{a b c}}{C_{F}^{3}}\left(\frac{11}{24}-\zeta_{3}\right)
\end{aligned}
$$

The numerical values of the Riemann $\zeta$ functions are $\zeta_{3}=1.2020569 \ldots$ and $\zeta_{5}=1.0369278 \ldots$. The coefficients $d^{a b c}$ are the symmetric structure constants of the gauge group. For SU(N) type theories one has $d^{a b c} d^{a b c} / C_{F}^{3}=16 x-6 x^{2}$.

\section{A.3 The Theoretical Prediction for $R_{l}$}

The theoretical prediction for $R_{l}$ is obtained from that for $R_{\gamma}$ by taking into account quark mass effects and the fact that, in the coupling of the primary quarks to the $Z$, vector and axial-vector currents contribute differently [99]. The prediction can be written as follows:

$$
R_{l}=\frac{\Gamma(Z \rightarrow \text { hadrons })}{\Gamma\left(Z \rightarrow l^{+} l^{-}\right)}=R_{l}^{e w}\left(1+\delta_{0}+\delta_{v}+\delta_{t}+\delta_{m}\right)
$$

Here $R_{l}^{e w}$ is the purely electro-weak prediction without QCD corrections, $\delta_{0}$ is the QCD correction for the case of massless quarks which is common to the vector and the axial current, while $\delta_{v}$ is an additional term which only contributes to the vector current. The two remaining terms are mass corrections, $\delta_{t}$ a correction in the axial current due to the large mass splitting between top and bottom quark mass, and $\delta_{m}$ the modification of the QCD correction due to the finite quark masses.

Using the effective parametrization of both the top and the Higgs mass dependence from the TOPAZ0 program [193] given in [44] one obtains

$$
R_{l}^{e w}=19.995\left(1-2.2 \cdot 10^{-4} \ln \left(\frac{M_{H}}{M_{Z}}\right)^{2}\right)\left(1-4.7 \cdot 10^{-4}\left(\frac{M_{t}}{M_{Z}}\right)^{2}\right)
$$

where the coefficient 19.995 was chosen such that the effective formula given in [44] is reproduced as closely as possible.

With the functions defined in the theoretical prediction for $R_{\gamma}$ and defining $r_{q}^{v}$ and $r_{q}^{a}$ as the relative production rates of quarks of type $q$ via the vector and axial vector current respectively, one has for $\delta_{0}$ and $\delta_{v}$ :

$$
\begin{aligned}
& \delta_{0}=K_{1} a+K_{2} a^{2}+\left(K_{3}+R_{3}\right) a^{3} \\
& \delta_{v}=\left(T_{3} a^{3}\right) \cdot\left(\frac{\left(\sum_{q} v_{q}\right)^{2}}{3 \sum_{q} v_{q}^{2}}\right) \sum_{q} r_{q}^{v}
\end{aligned}
$$

With $v_{q}$ and $a_{q}$ the vector and axial vector couplings of quarks $q$ to the $Z$,

$$
v_{q}=I_{3}^{q}-2 Q_{q} \sin ^{2} \theta_{w} \text { and } a_{q}=I_{3}^{q}
$$

the relative production rates are given by [194]

$$
r_{q}^{v}=N \cdot v_{q}^{2} \beta_{q} \frac{3-\beta_{q}^{2}}{2} \quad \text { and } \quad r_{q}^{a}=N \cdot a_{q}^{2} \beta_{q}^{3},
$$


where the velocity $\beta_{q}$ in the threshold factors depends on the pole-mass $M_{q}$ of the quarks as $\beta_{q}^{2}=1-4 M_{q}^{2} / M_{Z}{ }^{2}$. The normalization $N$ is fixed by the condition $\sum_{q}\left(r_{q}^{v}+r_{q}^{a}\right)=1$.

The top mass correction $\delta_{t}$ has been calculated in [195]. The leading order term from the triangle anomaly has the colour structure $\left(T_{j i}^{a} T_{i j}^{b}\right)\left(T_{l k}^{a} T_{k l}^{b}\right)=T_{F}^{2} N_{A}$, which can be rewritten using the identity $N_{A}=N_{F} C_{F} / T_{F}$ to yield $T_{F} C_{F} N_{F}$. Here $N_{F}=3$ is the number of quark degrees of freedom. With this, one obtains

$$
\delta_{t}=-a^{2} r_{b}^{a} y\left[\frac{37}{12}-12 \ln \frac{M_{Z}}{M_{t}}-\frac{14}{27}\left(\frac{M_{Z}}{M_{t}}\right)^{2}\right] .
$$

The next term in this expression is $\mathcal{O}\left(M_{Z} / M_{t}\right)^{4}$ and already negligible compared to the uncertainty in this correction from the error in the top mass. The next-to-leading order term in $\delta_{t}$ proportional to $a^{3}$ is known [196], but amounts to only $15 \%$ of this leading order correction and will be ignored in the following.

The leading order mass correction $\delta_{m}$ expressed as function of the pole mass of the quarks is given by

$$
\delta_{m}=a \sum_{q} 18 \frac{M_{q}^{2}}{M_{Z}^{2}}\left(r_{q}^{v}-r_{q}^{a} \ln \frac{M_{q}^{2}}{M_{Z}^{2}}\right) .
$$

An improved mass correction is obtained by absorbing large logarithms into running masses $\bar{m}_{q}$ [197]. Evaluated at the scale $M_{Z}$, it is conveniently written in the following form:

$$
\delta_{m}=\sum_{q} 6 \frac{M_{q}^{2}}{M_{Z}^{2}}\left\{r_{q}^{a}\left(1-\frac{\bar{m}_{q}^{2}}{M_{q}^{2}}\right)+a\left[3 r_{q}^{v} \frac{\bar{m}_{q}^{2}}{M_{q}^{2}}-r_{q}^{a}\left(K_{1}+\frac{11}{2} \frac{\bar{m}_{q}^{2}}{M_{q}^{2}}\right)\right]\right\}
$$

\section{A.4 The Theoretical Prediction for $R_{\tau}$}

The theoretical prediction for $R_{\tau}$ is also related to $R_{\gamma}$. Detailed discussions can be found in $[49,50]$. It can be written in the following form:

$$
R_{\tau}=\frac{\Gamma\left(\tau^{-} \rightarrow \nu_{\tau} \text { hadrons }\right)}{\Gamma\left(\tau^{-} \rightarrow \nu_{\tau} \bar{\nu}_{e} e^{-}\right)}=R_{\tau}^{E W}\left(1+\delta_{E W}+\delta_{0}+\delta_{c}+\delta_{m}\right)
$$

Here $R_{\tau}^{E W}=3.0582$ denotes the purely electro-weak expectation, which is modified by a residual correction $\delta_{E W}=0.001$. The dominant correction is the term $\delta_{0}$, which for vanishing quark masses again can be calculated in perturbative QCD. The additional terms $\delta_{c}$ and $\delta_{m}$ are the non-perturbative corrections from vacuum expectation values and mass corrections, respectively. A detailed description of the various terms can be found in the literature. Only a short summary will be presented here.

The main difference to the case of $R_{l}$ is the fact that the hadronic system produced in $\tau$ decays is not at a fixed mass but rather exhibits a mass spectrum ranging from $M_{\pi}$ to $M_{\tau}$. As a consequence, the QCD correction to the hadronic width is obtained by integrating the correction to $R_{\gamma}$ over the mass spectrum. Expressing the running coupling constant through its value at the scale $M_{\tau}$ and turning the integral over the mass spectrum into a contour integral one obtains [50]:

$$
\delta_{Q C D}=K_{1} A_{1}+K_{2} A_{2}+K_{3} A_{3}+\ldots
$$

with

$$
A_{n}=\frac{1}{2 \pi i} \oint_{|s|=M_{\tau}^{2}} \frac{d s}{s}\left(1-2 \frac{s}{M_{\tau}^{2}}+2 \frac{s^{3}}{M_{\tau}^{6}}-4 \frac{s^{4}}{M_{\tau}^{8}}\right) a^{n}(-s),
$$


where $a(-s)$ and $a\left(M_{\tau}\right)$ are related via Eq. (88).

The correction $\delta_{c}$ can be expanded in powers of $1 / M_{\tau}$ as

$$
\delta_{c}=\frac{B_{4}}{M_{\tau}^{4}}+\frac{B_{6}}{M_{\tau}^{6}}+\frac{B_{8}}{M_{\tau}^{8}}+\ldots
$$

A detailed analysis of the leading term $B_{4}$, giving its composition in terms of the strong coupling constant and the vacuum expectation values of the quark and gluon fields is given in [49]:

$$
B_{4}=\frac{11}{4} \alpha_{s}^{2}\left\langle\frac{\alpha_{s}}{\pi} G G\right\rangle-\left(16 \pi^{2}+54 \alpha_{s}^{2}\right)\left\langle\hat{m} \hat{\mu}^{3}\right\rangle_{u d s}+8 \alpha_{s}^{2} \sum_{u d s} \hat{m} \hat{\mu}^{3}-\frac{48 \pi}{\alpha_{s}}\left\langle\bar{m}^{4}\right\rangle_{u d s}
$$

The notation $\langle X\rangle_{u d s}$ is defined as a weighted sum over the variable $X$ for $u, d$ and $s$ quarks $\langle X\rangle_{u d s}=X_{u}+c_{c}^{2} X_{d}+s_{c}^{2} X_{s}$, where $c_{c}$ and $s_{c}$ are the cosine and sine of the Cabbibo angle, respectively. The exact definition and physical meaning of the various condensates can be found in [49]. The inverse power of the coupling constant appearing in $B_{4}$ arises from factorizing logarithms of a quark mass into the quark and gluon condensates [198]. The numerical values of the terms $B_{6}$ and $B_{8}$ are available from phenomenological fits to different data sets. Taking the results from [49] and setting $\alpha_{s}=0.36$ the numerical value of $\delta_{c}$ becomes:

$$
\delta_{c}=-0.011
$$

Finally the mass corrections $\delta_{m}$ must be determined. A detailed discussion is given in [49]. Collecting all mass corrections which are independent of any vacuum expectation values one finds,

$$
\delta_{m}=-\frac{8}{M_{\tau}^{2}}\left\langle\bar{m}^{2}\right\rangle_{u d s}(1+8 a)+\frac{1}{M_{\tau}^{4}}\left(24 \bar{m}_{u}^{2}\left\langle\bar{m}^{2}\right\rangle_{d s}+\frac{27}{4}\left\langle\bar{m}^{4}\right\rangle_{u d s}\right),
$$

with $\langle X\rangle_{d s}=c_{c}^{2} X_{d}+s_{c}^{2} X_{s}$. All running masses and the coupling constant $a$ must be evaluated at the scale $M_{\tau}$. 


\section{References}

[1] J. D. Bjorken and E. A. Paschos, Phys. Rev. 185 (1969) 1975.

[2] M. Y. Han and Y. Nambu, Phys. Rev. B139 (1965) 1006;

E. Reya, Phys. Rep. 69 (1981) 195.

[3] H. Fritzsch, M. Gell-Mann and H. Leutwyler, Phys. Lett. B47 (1973) 365.

[4] D. J. Gross and F. Wilczek, Phys. Rev. Lett. 30 (1973) 1343.

[5] H. D. Politzer, Phys. Rev. Lett. 30 (1973) 1346.

[6] D. Buskulic et al., ALEPH collaboration, Phys. Lett. B303 (1993) 198.

[7] B. R. Webber, Phys. Lett. B339 (1994) 148;

Yu. L. Dokshitzer and B. R. Webber, Phys. Lett. B352 (1995) 451;

P. Nason and M. Seymour, Nucl. Phys. B454 (1995) 291;

R. Akhoury and V. I. Zakharov, Phys. Lett. B357 (1995) 646; Nucl. Phys. B465 (1996) 295 ;

M. Beneke and V. M. Braun, Nucl. Phys. B454 (1995) 253.

[8] M. Gell-Mann, Phys. Lett. 8 (1964) 214.

[9] G. Zweig, CERN-TH 401 and TH 412 (1964).

[10] E. D. Bloom et al., Phys. Rev. Lett. 23 (1969) 930;

M. Breidenbach et al., Phys. Rev. Lett. 23 (1969) 935.

[11] R. P. Feynman, Photon Hadron Interactions, Benjamin, New York, 1972.

[12] P. M. Zerwas and H. A. Kastrup, eds., QCD 20 Years Later, World Scientific, Singapore (1993).

[13] G. Altarelli, Phys. Rep. 81 (1982) 1.

[14] D. H. Perkins, Introduction to High Energy Physics, Addison-Wesley, Menlo Park, California, 1987.

[15] J. Ellis, M. K. Gaillard, G. Ross Nucl. Phys. B111 (1976) 253.

[16] R. K. Ellis, D. A. Ross and A. E. Terrano, Nucl. Phys. B178 (1981) 421.

[17] A. Altarelli and G. Parisi, Nucl. Phys. B126 (1977) 298;

Yu. L. Dokshitzer, Sov. Phys. JETP 46 (1977) 641.

[18] B. I. Ermolaev, V. S. Fadin, ZhETF Pis'ma 33 (1981) 285;

A. H. Mueller, Phys. Lett. B104 (1981) 161;

V. S. Fadin, Yad. Fiz. 36 (1982).

[19] D. Decamp et al., ALEPH Collaboration, Nucl. Instr. Methods A294 (1990) 121.

[20] D. Buskulic et al., ALEPH Collaboration, Nucl. Instr. Methods A360 (1995) 481.

[21] G. Batignani et al., 1991 IEEE Nuclear Science Symposium, Santa Fe, IEEE. Trans. Nucl. Sci. NS-39(4/5) (1992) Vol. 1, p. 438. 
[22] G. J. Barber et al., Nucl. Instr. Methods A279 (1989) 212.

[23] W. B. Atwood et al., Nucl. Instr. Methods A306 (1991) 446.

[24] J. E. Campagne and R. Zitoun, Z. Phys. C43 (1989) 469.

[25] T. Sjöstrand, Comp. Phys. Comm. 82 (1994) 74.

[26] D. Buskulic et al., ALEPH Collaboration, Z. Phys. C55 (1992) 209.

[27] W. Bartel et al., JADE Collaboration, Z. Phys. C33 (1986) 23; S. Bethke et al., JADE Collaboration, Phys. Lett. B213 (1988) 235.

[28] Proceedings of the Durham Workshop, W. J. Stirling, J. Phys. G: Nucl. Part. Phys. 17 (1991) 1567

N. Brown and W. J. Stirling, Phys. Lett. B252 (1990) 657;

S. Bethke et al., Nucl. Phys. B370 (1992) 310.

[29] B. Andersson et al., Phys. Rep. 97 (1983) 31.

[30] L. Lönnblad, Comp. Phys. Comm. 71 (1992) 15.

[31] G. Marchesini et al., Comp. Phys. Comm. 67 (1992) 465.

[32] C. Peterson et al., Phys. Rev. D27 (1983) 105.

[33] G. Gustafson, Phys. Lett. B175 (1986) 453;

G. Gustafson, U. Pettersson, Nucl. Phys. B306 (1988) 746;

B. Anderson et al., Z. Phys. C43 (1989) 621;

B. Anderson, G. Gustafson, L. Lönnblad, Nucl. Phys. B339 (1990) 393.

[34] P. Abreu et al., DELPHI Collaboration, Z. Phys. C65 (1995) 587.

[35] D. Buskulic et al., ALEPH Collaboration, Z. Phys. C62 (1994) 1.

[36] D. Buskulic et al., ALEPH Collaboration, Z. Phys. C62 (1994) 179.

[37] D. Buskulic et al., ALEPH Collaboration, Phys. Lett. B357 (1995) 699.

[38] D. Buskulic et al., ALEPH Collaboration, Z. Phys. C69 (1996) 393.

[39] G. Hanson et al., Phys. Rev. Lett. 35 (1975) 1609.

[40] R. Brandelik et al., TASSO Collaboration, Phys. Lett. B97 (1980) 453;

Ch. Berger et al., PLUTO Collaboration, Phys. Lett. B97 (1980) 459.

[41] E. Laermann, K.H. Streng, P.M. Zerwas, Z. Phys. C3 (1980), 289; Erratum Z. Phys. C52 (1991) 352.

[42] M. A. Shifman, A. L. Vainshtein and V. I. Zakharov, Nucl. Phys. B147 (1979) 385.

[43] L. R. Surguladze and M. A. Samuel, Phys. Rev. Lett. 66 (1991) 560;

S. G. Gorishny, A. L. Kataev and S. A. Larin, Phys. Lett. B259 (1991) 144.

[44] T. Hebbeker et al., Phys. Lett. B331 (1994) 165. 
[45] D. Buskulic et al., ALEPH Collaboration, Z. Phys. C62 (1994) 539.

[46] J. Alcaraz et al., The LEP Electroweak Working Group and the SLD Heavy Flavour Group, LEPEWWG/96-02.

[47] M. Martinez et al., Z. Phys. C49 (1991) 645.

[48] D. Buskulic et al., ALEPH Collaboration, Phys. Lett. B307 (1993) 209.

[49] E. Braaten, S. Narison and A. Pich, Nucl. Phys. B373 (1992) 581.

[50] F. Le Diberder and A. Pich, Phys. Lett. B286 (1992) 147; ibid. 289 (1992) 165.

[51] D. Buskulic et al., ALEPH Collaboration, Z. Phys. C70 (1996) 561.

[52] D. Buskulic et al., ALEPH Collaboration, Z. Phys. C70 (1996) 549.

[53] D. Buskulic et al., ALEPH Collaboration, Z. Phys. C70 (1996) 579.

[54] L. Duflot, in Proceedings of the QCD 94 Conference, ed. S. Narison, Nucl. Phys. (Proc. Suppl.) 39B (1995) 322.

[55] W. Bernreuther and W. Wetzel, Nucl. Phys. B197 (1982) 228.

[56] A. Pich, in Proceedings of the QCD 94 Conference, ed. S. Narison, Nucl. Phys. (Proc. Suppl.) 39B (1995) 326.

[57] P. Ball, M. Beneke and V. M. Braun, Nucl. Phys. B452 (1995) 563.

[58] G. Altarelli, P. Nason and G. Ridolfi, Z. Phys. C68 (1995) 257.

[59] Z. Kunszt et al., in Proceedings of the Workshop on Z Physics at LEP, eds. G. Altarelli, R. Kleiss and C. Verzegnassi, CERN Report 89-08, p. 373-453.

[60] T. Sjöstrand and M. Bengtsson, Comp. Phys. Comm. 43 (1987) 367.

[61] M. Bengtsson and T. Sjöstrand, Phys. Lett. B185 (1987) 435.

[62] G. Marchesini and B. Webber, Cavendish-HEP-88/7; Nucl. Phys. B310 (1988) 461;

I. Knowles, Nucl. Phys. B310 (1988) 571.

[63] D. Decamp et al., ALEPH Collaboration, Phys. Lett. B255 (1991) 623.

[64] D. Decamp et al., ALEPH Collaboration, Phys. Lett. B257 (1991) 479.

[65] P. Nason, program EVENT, private communication.

[66] S. Catani et al., Nucl. Phys. B407 (1993) 3, and references therein.

[67] S. Catani et al., Phys. Lett. B263 (1991) 491.

[68] S. Catani, G. Turnock and B. R. Webber, Phys. Lett. B272 (1991) 368.

[69] S. Catani et al., Phys. Lett. B269 (1991) 432.

[70] G. Dissertori and M. Schmelling, Phys. Lett. B361 (1995) 167. 
[71] D. Decamp et al., ALEPH Collaboration, Phys. Lett. B284 (1992) 163.

[72] D. Buskulic et al., ALEPH Collaboration, Phys. Lett. B357 (1995) 487; and Erratum, ibid. B364 (1995) 247.

[73] P. Nason and B. R. Webber, Nucl. Phys. B421 (1994) 473.

[74] G. Altarelli, R. K. Ellis, G. Martinelli and S.-Y. Pi, Nucl. Phys. B160 (1979) 301.

[75] V. N. Gribov and L. N. Lipatov, Sov. J. Nucl. Phys. 15 (1972) 78;

G. Altarelli, G. Parisi, Nucl. Phys. B126 (1977) 298;

Yu. L. Dokshitzer, Sov. Phys. JETP 46 (1977) 641.

[76] G. Gurci, W. Furmanski and R. Petronzio, Nucl. Phys. B175 (1980) 27;

W. Furmanski and R. Petronzio, Phys. Lett. B97 (1980) 437.

[77] D. Buskulic et al., ALEPH Collaboration, Phys. Lett. B313 (1993) 535.

[78] D. Buskulic et al., ALEPH Collaboration, Phys. Lett. B313 (1993) 549.

[79] D. Decamp et al., ALEPH Collaboration, Phys. Lett. B273 (1991) 181.

[80] W. Braunschweig et al., TASSO Collaboration, Z. Phys. C47 (1990) 187.

[81] A. Petersen et al., MARK II Collaboration, Phys. Rev. D37 (1988) 1.

[82] H. Aihara et al., TPC/2 $\gamma$ Collaboration, Phys. Rev. Lett. 61 (1988) 1263; updated in G. D. Cowan, internal note TPC-LBL-94-01.

[83] O. Podobrin, CELlO Collaboration, Ph. D. thesis, Universität Hamburg. See also ref. [85].

[84] Y. K. Li et al., AMY Collaboration, Phys. Rev. D41 (1990) 2675.

[85] P. Abreu et al., DELPHI Collaboration, Phys. Lett. B311 (1993) 408.

[86] See, for instance, Yu. L. Dokshitzer et al., Basics of Perturbative QCD, Editions Frontières, Gif-sur-Yvette (France) 1991, and references therein.

[87] C. P. Fong and B. R. Webber, Nucl. Phys. B355 (1991) 54.

[88] M. Schmelling, Status of the strong coupling constant, plenary talk at the XXVIII International Conference on High Energy Physics, Warsaw, July 1996. To appear in the Proceedings.

[89] R. M. Barnett et al., Particle Data Group, Phys. Rev. D54 (1996) 1.

[90] S. Catani, G. Turnock and B. R. Webber, Phys. Lett. B295 (1992) 269.

[91] W. Braunschweig et al., TASSO Collaboration, Z. Phys. C47 (1990) 181.

[92] B. Adeva et al., L3 Collaboration, Phys. Lett. B263 (1991) 551.

[93] P. Abreu et al., DELPHI Collaboration, Phys. Lett. B274 (1992) 498.

[94] M. H. Seymour, program EVENT2, private communication;

S. Catani and M. H. Seymour, Phys. Lett. B378 (1996) 287. 
[95] B. Lampe, Phys. Lett. B301 (1993) 435.

[96] R. D. Schamberger et al., CUSB Collaboration, Phys. Lett. B138 (1984) 225;

S. E. Csorna et al., CLEO Collaboration, Phys. Rev. Lett. 56 (1986) 1221;

H. Albrecht et al., ARGUS Collaboration, Phys. Lett. B199 (1987) 291;

W. Kwong et al., Phys. Rev. D37 (1988) 3210;

W. Braunschweig et al., TASSO Collaboration, Z. Phys. C44 (1989) 365;

A. Geiser, UA1 Collaboration, in Proceedings of the 27th Rencontre de Moriond: QCD and High Energy Hadronics Interactions, Les Arcs, France, ed. J. Tran Thanh Van (Editions Frontières) (1991) p. 159 ff.

[97] B. Adeva et al., L3 Collaboration, Phys. Lett. B271 (1991) 461;

R. Akers et al., OPAL Collaboration, Z. Phys. C60 (1993) 397;

P. Abreu et al., DELPHI Collaboration, Phys. Lett. B307 (1993) 221;

R. Akers et al., OPAL Collaboration, Z. Phys. C65 (1995) 31;

K. Abe et al., SLD Collaboration, Phys. Rev. D53 (1996) 2271.

[98] D. Buskulic et al., ALEPH Collaboration, Phys. Lett. B355 (1995) 381.

[99] T. Hebbeker, PITHA 91/08; Phys. Rep. 217 (1991) 69.

[100] J.G. Körner, G. Schierholz and J. Willrodt, Nucl. Phys. B185 (1981) 365;

K.J.F. Gaemers and J.A.M. Vermaseren, Z. Phys. C7 (1980) 81;

D. Danckaert et al., Phys. Lett. B114 (1982) 203.

[101] ALEPH Collaboration, D. Decamp et al., Phys. Lett. B284 (1992) 151.

[102] J.M. Scarr, I. ten Have, ALEPH-NOTE 89 - 150.

[103] R. Tarrach, Nucl. Phys. B183 (1981) 384.

[104] F. Abe et al., CDF Collaboration, Phys. Rev. Lett. 74 (1995) 2626;

S. Abachi et al., D0 Collaboration, Phys. Rev. Lett. 74 (1995) 2632.

[105] See, for instance, I. Antoniadis, J. Ellis, D.V. Nanopoulos, Phys. Lett. B262 (1991) 109.

[106] M. G. Alviggi et al., LEP Electroweak Working Group, LEPEWWG 95-01.

[107] Ya. I. Azimov et al., Z. Phys. C27 (1985) 65; Z. Phys. C31 (1986) 213.

[108] C. P. Fong and B. R. Webber, Phys. Lett. B229 (1989) 289.

[109] D. Buskulic et al., ALEPH Collaboration, CERN-PPE/96-43 (1996).

[110] M. Z. Akrawy et al., OPAL Collaboration, Phys. Lett. B247 (1990) 617.

[111] B. Adeva et al., L3 Collaboration, Phys. Lett. B259 (1991) 199.

[112] M. Chmeisanni, I.F.A.E., Universitat Autonoma de Barcelona, Annual Report (1992) 49.

[113] R. Odorico, University of Bologna report DFUB 92-6 (1992).

[114] M. Acciarri et al., L3 Collaboration, Phys. Lett. B353 (1995) 145.

[115] Yu. L. Dokshitzer et al., Phys. Lett. B245 (1990) 243. 
[116] Yu. L. Dokshitzer, G. Marchesini, G. Oriani, Nucl. Phys. B387 (1990) 675.

[117] P. D. Acton et al., OPAL Collaboration, Z. Phys. C58 (1992) 207.

[118] W. Bartel et al., JADE Collaboration, Phys. Lett. B101 (1981) 129; Z. Phys. C21 (1983) 37 .

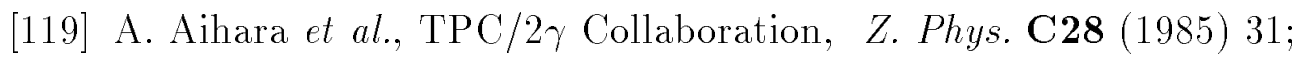

M. Althoff et al., TASSO Collaboration, Z. Phys. C29 (1985) 29;

P. D. Sheldon et al., MARK II Collaboration, Phys. Rev. Lett. 57 (1986) 1398;

P. Aarnio et al., DELPHI Collaboration, Phys. Lett. B240 (1990) 271;

M. Z. Akrawy et al., OPAL Collaboration, Phys. Lett. B261 (1991) 334.

[120] Z. Koba, H. B. Nielsen, P. Olesen, Nucl. Phys. B40 (1972) 317.

[121] E. D. Malaza, B. R. Webber, Phys. Lett. B149 (1984) 501;

E. D. Malaza, B. R. Webber, Nucl. Phys. B267 (1986) 702.

[122] S. Carius, G. Ingelmann, Phys. Lett. B252 (1990) 647;

R. Szwed, G. Wrochna, Z. Phys. C47 (1990) 447;

R. Szwed, G. Wrochna, A. K. Wróblewski, Mod. Phys. Lett. A5 (1990) 981.

[123] D. Buskulic et al., ALEPH Collaboration, Z. Phys. C69 (1995) 15

[124] V. Blobel, DESY 84-118 (1984).

[125] V. B. Anikeev and V. P. Zhigunov, Phys. Part. Nucl. 24 (4) (1993) 424.

[126] M. Schmelling, Nucl. Instr. Methods A340 (1994) 400.

[127] R. P. Feynman, Phys. Rev. Lett. 23 (1969) 1415.

[128] W. Braunschweig et al., TASSO Collaboration, Z. Phys. C45 (1989) 193.

[129] D. Bender et al., HRS Collaboration, Phys. Rev. D31 (1985) 1.

[130] P. Abreu et al., DELPHI Collaboration, Z. Phys. C50 (1991) 185.

[131] W. Bartel et al., JADE Collaboration, Z. Phys. C20 (1983) 187.

[132] M. Schmelling, Physica Scripta 51 (1995) 683.

[133] R. Akers et al., OPAL Collaboration, Z. Phys. C61 (1994) 209.

[134] R. E. Ansorge et al., UA5 Collaboration, Z. Phys. C43 (1989) 357.

[135] P. Abreu et al., DELPHI Collaboration, Z. Phys. C52 (1991) 511.

[136] A. Giovannini and L. Van Hove, Z. Phys. C30 (1986) 391.

[137] Ya. B. Zel'dovich et al., Sov. Phys. Usp. 30 (1987) 353.

[138] A. Bialas and R. Peschanski, Nucl. Phys. B273 (1986) 703.

[139] D. Decamp et al., ALEPH Collaboration, Z. Phys. C53 (1992) 21. 
[140] V. Raab, "Intermittenz bei $91 \mathrm{GeV}$ in der Reaktion $e^{+} e^{-} \rightarrow$ Hadronen," Diplomarbeit an der Technischen Universität München (1991), MPI-PhE/92-06.

[141] W. Ochs and J. Wosiek, Phys. Lett. B68 (1993) 144;

Yu. L. Dokshitzer and C. M. Dremin, Nucl. Phys. B402(1993)139;

Ph. Brax, J. L. Meunier and R. Peschanski, Z. Phys. C62 (1994) 649;

W. Ochs and J. Wosiek, Z. Phys. C68 (1995) 269.

[142] S. Catani et al., Nucl. Phys. B377 (1992) 445; Nucl. Phys. B383 (1992) 419;

[143] R. Akers et al., OPAL Collaboration, Z. Phys. C63 (1994) 363.

[144] O. Adriani et al., L3 Collaboration, Phys. Rep. 236 (1993) 207.

[145] B. Webber, private communication.

[146] D. Buskulic et al., ALEPH Collaboration, Phys. Lett. B346 (1995) 389.

[147] M. Z. Akrawy et al., OPAL Collaboration, Phys. Lett. B261 (1991)334;

R. Akers et al., OPAL Collaboration, Z. Phys. C68 (1995) 179;

P. Abreu et al., DELPHI Collaboration, Z. Phys. C70 (1996) 179;

D. Buskulic et al., ALEPH Collaboration, Phys. Lett. B346 (1995) 389.

[148] M. Derrick et al., HRS Collaboration, Phys. Lett. B165 (1985) 449;

P. Abreu et al., DELPHI Collaboration, Z. Phys. C56 (1992) 63.

[149] D. Buskulic et al., ALEPH Collaboration, Nucl. Instr. Methods A346 (1994) 461;

[150] D. Decamp et al., ALEPH Collaboration, Phys. Lett. B266 (1991) 218.

[151] D. Buskulic et al., ALEPH Collaboration, Phys. Lett. B352 (1995) 479.

[152] D. Buskulic et al., ALEPH Collaboration, Phys. Lett. B384 (1996) 353.

[153] R. Akers et al., OPAL Collaboration, Phys. Lett. B352 (1995) 176.

[154] G. Alexander et al., OPAL Collaboration, Z. Phys. C69 (1996) 543.

[155] P. D. Acton et al., OPAL Collaboration, Z. Phys. C58 (1993) 405;

D. Buskulic et al., ALEPH Collaboration, Z. Phys. C57 (1993) 17;

P. Abreu et al., DELPHI Collaboration, Z. Phys. C53 (1992) 555;

O. Adriani et al., L3 Collaboration, Phys. Lett. B292 (1992) 472.

[156] E. W. N. Glover and W. J. Stirling, Phys. Lett. B295 (1992) 128.

[157] G. Kramer and H. Spiesberger, "Matrix element calculation of quark bremsstrahlung in $\mathcal{O}\left(\alpha \alpha_{\mathcal{S}}\right)$ ", DESY 92-022 (1992), contribution to the Workshop on Photon Radiation from Quarks, Annecy, France, 1991.

[158] E. W. N. Glover and A. G. Morgan, Z. Phys. C62 (1994) 311.

[159] The LEP Collaborations and the LEP Electroweak Working Group, CERN-PPE/93-157.

[160] D. Buskulic et al., ALEPH Collaboration, Z. Phys. C69 (1996) 365. 
[161] D. Decamp et al., ALEPH Collaboration, Z. Phys. C66 (1995) 355.

[162] R. Akers et al., OPAL Collaboration, Z. Phys. C63 (1994) 181.

[163] R. Itoh et al., Phys. Rev. Lett. 345 (1995) 335.

[164] M. Althoff et al., TASSO Collaboration, Z. Phys. C17 (1983) 5.

[165] W. Braunschweig et al., TASSO Collaboration, Z. Phys. C42 (1989) 189.

[166] H. Aihara et al., TPC Collaboration, Phys. Rev. Lett. 61 (1988) 1263.

[167] G. D. Cowan, University of California, LBL-24715 (1988).

[168] R. Barate et al., ALEPH Collaboration, CERN-PPE/96-168.

[169] D. Decamp et al., ALEPH Collaboration, Phys. Lett. B292 (1992) 210.

[170] D. Buskulic et al., ALEPH Collaboration, Z. Phys. C69 (1996) 379.

[171] D. Decamp et al., ALEPH Collaboration, Z. Phys. C64 (1994) 361.

[172] P. D. Acton et al., OPAL Collaboration, Phys. Lett. B291 (1993) 503.

[173] M. Baubillier et al., Z. Phys. C23 (1984) 213.

[174] B. Anderson et al., Lund Report LU TP 84-9 (1984).

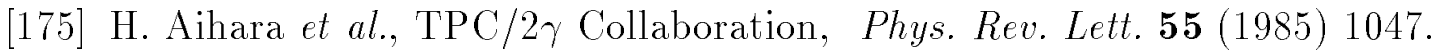

[176] H. Albrecht et al., ARGUS Collaboration, Z. Phys. C43 (1989) 45.

[177] B. R. Webber, private communication.

[178] D. Decamp et al., ALEPH Collaboration, Z. Phys. C54 (1992) 75.

[179] M. G. Bowler, Z. Phys. C29 (1985) 617.

[180] G. I. Kopylov, M. I. Podgoretskii, Sov. J. Nucl. Phys. 18 (1974) 336 and Sov. J. Nucl. Phys. 19 (1974) 215.

[181] R. Lednicky, V. L. Lyuboshits, Sov. J. Nucl. Phys. 35 (1982) 770.

[182] M. G. Bowler, OUNP-91-23 (1991).

[183] H. Aihara et al., TPC-PEP4 Collaboration, Phys. Rev. D31 (1985) 996.

[184] P. Avery et al., CLEO Collaboration, Phys. Rev. D32 (1985) 2294.

[185] M. Althoff et al., TASSO Collaboration, Z. Phys. C30 (1986) 355.

[186] I. Juričić et al., Mark II Collaboration, Phys. Rev. D39 (1989) 1.

[187] P. D. Acton et al., OPAL Collaboration, Phys. Lett. B298 (1993) 456.

[188] P. Abreu et al., DELPHI Collaboration, Z. Phys. C63 (1994) 17. 
[189] D. R. T. Jones, Nucl. Phys. B74 (1974) 531;

D. Caswell, Phys. Rev. Lett. 33 (1974) 244.

[190] O. Tarasov, A. Vladimirow and A. Zharkov, Phys. Lett. B93 (1980) 429.

[191] O. Nachtmann and W. Wetzel, Nucl. Phys. B187 (1981) 333.

[192] W. Bernreuther and W. Wetzel, Nucl. Phys. B197 (1982) 228;

W. Bernreuther, Annals of Physics 151 (1983) 127;

W. J. Marciano, Phys. Rev. D29 (1984) 580;

G. Rodrigo and A. Santamaria, CERN-TH.6899/93.

[193] G. Montagna et al., Nucl. Phys. B401 (1993) 3, Comp. Phys. Comm. 76 (1993) 328.

[194] A. Djouadi, J. H. Kühn and P. M. Zerwas, Z. Phys. C46 (1990) 411.

[195] B. A. Kniehl and J. H. Kühn, Phys. Lett. B224 (1989) 229;

B. A. Kniehl and J. H. Kühn, Nucl. Phys. B329 (1990) 547.

[196] K. G. Chetyrkin and O. V. Tarasov, Phys. Lett. B327 (1994) 114.

[197] K. G. Chetyrkin and J. H. Kühn, Phys. Lett. B248 (1990) 359;

K. G. Chetyrkin, J. H. Kühn and A. Kwiatkowski, Phys. Lett. B282 (1992) 221.

[198] S. C. Generalis, J. Phys. G15 (1989) L225. 